



Estr. dalle Memorie della Reale Accademia delle Scienze di Torino. Serie II, ToM. XXXVII

Torino, Stamperia Reale. 


\section{GATALOGO DESCRITTIVO}

\section{DEI \\ T A L A S S T E R I I}

RINVENUTI

NeI terrent teralapil deL PIEMONTE E DELLA LIGURIA

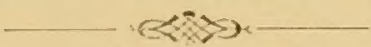

NOTICE

AFTER CAREFUL EXAMINATION OF THE NNER MARGIN AND TYPE OF MATERIAL VE HAVE SEWN THIS VOLUME BY HAND SO IT CAN BE MORE EASILY OPENED AND READ. er allo studio delle ossa fossili te in Italia; e se prima di quel per la coltura, vuoi per la viabi3 scoprendosi, venivano in gran ro o scomparendo sotto la marra ù come curiosità o prodigi, conza, dato l'allarme e spiegata con

fatti naturali la presenza di ossa di animali nei terreni superficiali, non tardarono gli scienziati ad interessarsi alle varie scoperte di scheletri che si erano fatte avanti ai loro tempi o che andavano man mano facendosi. L'interesse dimostrato a scoprimenti di simil genere per parte di coloro che successivamente furono a capo delle Collezioni di Storia Naturale di Torino produsse in breve anche nel volgo i suoi ottimi effetti. I coltivatori vedendo come ben soventi, dopo che essi avevano trovato un qualche avanzo di gigante e se ne era sparsa la nuova, non tardava a giungere sul luogo, ed affrontando per questo solo scopo lunghe ore di carrozza o di cammino, un Allioni, un Borson, od un Sismonda, ammaestrati da loro che $\mathrm{i}$ giganti erano da relegarsi tra le favole e che le ossa dissepolte 



\section{CATALOGO DESCRITTIVO}

\section{DEI \\ T A L A S S T ER I I}

RINTENTTI

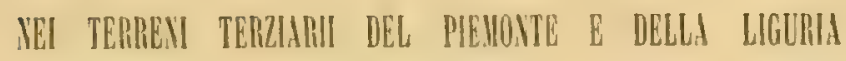

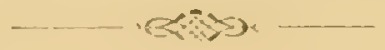

Limpulso dato in Francia dal Guvier allo studio delle ossa fossili non tardò ad apportare buoni effetti anche in Italia; e se prima di quel grande maestro le ossa fossili, che, vuoi per la coltura, vuoi per la vinbilitu, andavano man mano accidentalmente scoprendosi, venivano in gran parte religiosamente risepolte in luogo sacro o scomparendo sotto la marra del contadino e solo in minima parte, più come curiositì o prodigi, conservate nelle collezioni di lusso o di scienza, dato l'allarme e spiegata con fatti naturali la presenza di ossa di animali nei terreni superficiali, non tardarono gli scienziati ad interessarsi alle varie scoperte di scheletri che si erano fatte avanti ai loro tempi o che andavano man mano facendosi. L'interesse dimostrato a scoprimenti di simil genere per parte di coloro che successivamente furono a capo delle Collezioni di Storia Naturale di Torino produsse in breve anche nel volgo i suoi ottimi effetti. I coltivatori redendo come ben soventi, dopo che essi avevano trovato un qualche avanso di gigante e se ne era sparsa la nuova, non tardava a ginngere sul luogo, ed affrontando per questo solo scopo lunghe ore di carrozza o di cammino, un Allioni, un Borson, od un Sismonda, ammaestrati da loro che i giganti erano da relegarsi tra le favole e che le ossa dissepolte 
avevano appartenuto invece at antichi e talor strani animali, cessarono dalla pratica fino allora seguita di consegnare al cimitero o di fracassare barbaramente quanto incontravano nei loro lavori, ed invece ben soventi lo raccolsero, lo isolarono, lo ristorarono per quanto seppero ed o ne ornarono le loro casette o lo cedettero al Museo di Storia Naturale più in voga.

L'essere stata Torino per lungo tempo capitale dello Stato fece sì che ad essa convergessero preferibilmente, al pari delle forze vive, anche le curiosita naturali del piccolo Stato di cni era centro e che, nei limiti del possibile, si cercasse pure di raccogliere in essa quanto era necessario allo studio ed alla classificazione di quanto veniva man mano messo assieme sia per via di doni, sia per via di compre.

Cosi il Museo di Storia Naturale più in voga per tutto il Piemonte e per tutta la Liguria veniva ad essere quello di Torino; mentre in esso si raccoglievano tanti tesori relativi alla \%oologia ed alla Mineralogia, dai quali ancora oggidi si traggono nuovi ed utili insegnamenti sui prodotti naturali del nostro paese, mentre si andava pur lentamente formando una ricca serie di animali estinti trovati in tutti gli Stati Sardi di Terraferma, in questa acquistava sempre maggiore importanza e ricchezza la sezione degli avanzi di Vertebrati e sovratutto di Mammiferi fossili.

Dopo la felice scoperta del Mastodonte di Dusino, dopo lo scavo del medesimo e dopo la illustrazione fattane per opera dell' E. Sismonda, non passô più anno, si può dire, senza che i fratelli Sismonda od il Prof. Perez. od il Prof. Bellardi non venissero ripetutamente chiamati per andare a visitare ossani scoperti in qualche punto del piccolo regno. Quasi sempre essi ne tornarono con veri tesori; soventi anzi di tali tesori vennero direttamente e semplicemente portati a Torino dal fortunato scopritore, il quale quasi sempre fini per lasciare in Museo quello che aveva trovato, sia che si appagasse delle spiegazioni che gli venivano fornite e donasse senz'altro i pezzi allo Istituto, sia che in seguito a trattative si venisse ad un giusto apprezzamento sul valore dei pezzi portati.

Negli ultimi decenni però, non bastò al Prof. Gastaldi di aspettare che le ossa fossili venissero accidentalmente trovate ed ancora più accidentalmente scavate e salvate, ma percorrendo egli stesso passo a passo le nostre più celebri località fossilifere, procurò di trovare, far trovare e ricavare da esse quella maggior quantita di Vertebrati fossili che egli potè; procurò che i fossili venissero scavati colle norme più razionali e compatibili colle condizioni speciali delle varie località e dei varii pezzi, ed infine con un talento ed una abilità ingenite e perfezionate dallo studio e dalla 
lunga pratica, attese alla ristorazione ed alla conservazione dei pezzi fossili che gli renivano, per mezzo delle sue ricerche, alle mani incompleti o rotti. Egli è in tal guisa che mentre da un lato nel Museo di llineralogia e Geologia, che già si era individualizzato, si andava raccogliendo una gran quantità di ossami fossili del nostro paese, dall'altro si raccoglieva nel Museo della R. Scmola di Applicazione degli Ingegneri al Valentino, e con gravi sacrifizii materiali e pecuniarii fatti in proprio dal Prot. Gastaldi, una seconda partita di tali ossami, per molfitudine di pezzi, per rarita di specie e per grado di conservazione non certo inferiore alla prima. Allorquando nel 1878, dopochè i quattro Musei speciali, in cui si era scisso l'antico Museo di Storia Naturale di Torino, ebbero spazioso ed adatto locale nel Palazzo Garignano, fu stabilito nell'Universita l'insegnamento della Geologia con un Professore speciale e venne di tale insegnamento e della direzione del Museo Geologico incarisato il Prot. Gastaldi, questi aveva intenzione di sollecitare dal Regio Governo l'autorizzazione di far passare al Museo Geologico stesso quanto di relativo alla Paleontologia era stato da lui raccolto nella Collezione mineralonica della R. Suuola di Applicazione per gli Ingegneri con tondi affidatigli per le spese del Gabinetto, e di donare eziandio tutto quello, e non era piccola parte, che Denchè provisoriamente depositato nella Collezione, era però stato acquistato con fondi rroprii. Era intenzione del Prof. Gastaldi che alloraquando le due Collezioni paleontologiche fossero riunite in un corpo solo comprendente quasi tutto quello che era stato fino allora scoperto in Piemonte e gran parte di quanto proveniva dalla Liguria si facesse del medesimo un Catalogo ragionato e descrittivo, nel quale si registrassero tutti i pezzi e le specie già descritte citando lo opere in cui trovavasi l'illustrazione loro e si descrivessero ed illustrassero tutti quelli nuovi e non ancora conosciuti. Egli che avera con predilezione raccolti avanzi di Vertebrati fossili, desiderava che colla descrizione di quelli losse cominciato il Catalogo, e molte volte meco ne discorse: anzi, vedendo che io stesso cominciavo pure a rivolgere l'attenzione ai nostri Vertebrati, elbe alcune rolte a propormi di tosto intraprendere un tale lavoro.

Per l'improvvisa ed immatura morte del Prof. Gastaldi, molte pratiche da lui iniziate rimasero per qualche tempo in sospeso. Anche la Collezione paleontologica da lui raccolta non potè per alcuni anni passare al R. Museo Geologico e non vi fu congiunta che nel passato anno 1882.

Nell'autunno dello stesso 1882 i signori Proff. Baretti e Bellardi, sotto la cui direzione e condatta troransi le antiche Collezioni del R. Mnseo 
Geologico, accresciute delle nuove fatte dal Prof. Gastaldi, riconohbero la necessità di fare, specialmente per ciò che riguarda i Vertebrati, un Catalogo descrittivo, e con gentile pensiero vollero offrirmi di assumere un tale lavoro e di frequentare perciò il Museo, utilizzando pure la Biblioteca che si va in esso formando ed aumentandola di quelle opere la di cui necessità si venisse man mano dimostrando.

Ma appena dato uno sguardo allo insieme dei resti di Vertebraticonservati nel Museo, dovetti convincermi che allo stesso modo che grandissima era la quantità e varietà loro, così la loro determinazione ed illustrazione mi avrebbero tenuto più anni occupato, e che il Catalogo avrebbe assunte proporzioni tali da essere necessario il scinderlo in più parti. Ed allora immaginai di intraprendere una serie di Monogratie dei varii ordini di Vertebrati fossili rappresentati in Piemonte e Liguria, e come prima della serie tentai di intraprendere l'illustrazione dei Mammiferi marini che vissero da noi mentre nella valle del Po si deponevano i terreni terziarii superiori e medii, componendo cosi il Catalogo dei Cetacei e Sirenoidi fossili del Piemonte e della Liguria. In esso rennero considerati, oltre a tutti gli avanzi che si trovano ora nel Musen di Geologia di Torino, anche tutti quelli di cui potei conoscere l'esistenza e la provenienza piemontese o ligure e che, trorandosi conservati in altre Collezioni, mi furono gentilmente comunicati dai Proprietari e Direttori.

L'ordine adottato pel lavoro essendo, per non trascurare il benchè minimo pezzo, quello di Catalogo, si succedono, ripetuti talora un gran numero di volte, avanzi della stessa specie ma appartenenti ad individui differenti. Secondo l'opinione di distinti Paleontologi e che io trovo molto vera ed ho per conseguenza adottata, non esistono quasi mai veri doppi nella serie dei grandi Vertebrati fossili, ma ogni nuovo scheletro, ogni nuovo pezzo, anche appartenente ad una specie già conosciuta, può fornire utili cognizioni relative alle affinità ed alle abitudini delle specie. Quindi la conoseenza di alcune nostre specie risultera (come gia altre volte si praticò) più dal complesso delle descrizioni di porzioni di scheletri di più individui che non dalla descrizione minuta di un solo scheletro più completo e preso per tipo al quale riferire poi in massa tutti gli altri presentanti pochi caratteri principali considerati quali diagnostici della specie.

Soventi mi è capitato, nel corso di questo mio lavoro, di aver dinanzi pezzi già descritti da altri autori. Se, in tal caso, le descrizioni erano state fatte in lingua italiana ed in periodici, od opere od atti di Accademie italiane, facilmente alla mano degli studiosi del nostro paese, io ho sem- 
plicemente indicato dove esse si potevano trovare; se invece esse si tro. vavano in lavori pubblicati all'estero ed in lingue straniere, ho molto soventi riportate, traducendole, le descrizioni degli autori che se ne occuparono; e quando nuovi pezzi erano posteriormente sopravvenuti o gli antichi non erano stati, a mio credere, completamente interpretati, aggiunsi nuove descrizioni per darne al possibile piena conoscenza.

A completare finalmente il Catalogo ho stimato opportuno di dare le figure dei pezzi più interessanti e più adatti a mostrare i caratteri sui quali mi sono basato per stabilire non poche nuove specie o per estendere le fin qui incomplete cognizioni che si hanno su di altre.

Il lavoro è diviso in tre parti trattanti: la prima, dei Misticeti, la seconda, dei Denticeti, e la terzá, dei così detti Cetacei erbivori o Sirenoidi. Quest'ultima è straordinariamente piccina in confronto colle altre due; tale sproporzione dipende dal fatto che finora pochi sono gli avanzi di Sirenoidi trovati nel nostro paese e quelli già completamente esplicati, mentre molto più numerosi sono quelli che formano argomento delle due prime parti, e molti di essi o nuovi affatto o non completamente conosciuti.

Alla terza parte ho fatto succedere un riassunto generale dei generi e delle specie di Talassoterii del nostro paese, dimostrando quali fra di essi abbiano da noi vissuto nelle successive fasi del periodo terziario. Tale riassunto si trova poi raccolto per maggiore chiarezza e brevita in un solo quadro, nel quale in tante colonne, quante sono le più generalmente ammesse divisioni del nostro terziario, riunii le specie diverse di Talassoterii che vissero nel tempo corrispondente a ciascuna di esse.

Mentre rivolgo i più vivi ringraziamenti a clì mi propose e mi rese possibile questo lavoro, prego il benigno lettore a voler tener conto delle enormi difficolta necessariamente incontrate in questo genere di intrapresa ed a scusare le mende che forse avrà ad incontrare percorrendo questo Catalogo. 


\section{BIBLIOGRAFIA}

Mi sono servilo, per lo studio e per il confronto dei nostri Talassoterii con quelli viventi e con quelli fossili di altre regioni a noi più o meno vicine, degli scritti seguenti:

1. Van Beneden P. J. Quelques observations sur les fossiles de la province d'Anvers. Bulletin de 1'Acad. R. de Belg., $1^{\mathrm{e}}$ sẻr., tom. II, pag. 67; 8". Bruxelles 1835.

2. IDE.. Notes sur deux Cétacés fossiles provenant du bassin d'Anvers. Bull. d. 1'Acad. R. d. Belg., $1^{\text {e }}$ sér., tom. XIII, $1^{\text {re }}$ Part., pag. 257; 8. Bruxelles 1846 .

3. IDEs. Rapport sur la técouverte tossements fossiles faite ì Saint-Nicolas. Bull. d. l'Acad. R. d. Belg., $1^{\text {e }}$ sér., tom. VIII, pag. 123-148 (pag. 107$12 \%$, Rapports de MI. Nyst et de Koninck sur le même sujet); 8\%. Bruxelles 1859.

4. IDEx. Note sur un Cétuce troné mort en mer (Globicephatus melus). Bull. d. l'Acad. R. d. Belg., $2^{\text {e }}$ sér., tom. VIII, pag. 312-14; 8. Bruxelles 1859 .

3. IDEx. Rapport sur des ossements fossites trouve's dans les environs de SaintVicolas (Iperoodon, Plesiocetus, Dioplodon). Bull. l. l'Acad. R. d. Belg., $2^{e}$ sér., tom. X, pag. $403-410$ (pag. $401-402$, Communication de II. le $\mathrm{D}^{\mathrm{r}}$. V. Raemdonck et rapport de M. Nyst); 80. Bruxelles 1860.

6. Idexs. Un ILemmifère nouvenu du Crag d'Aneers (Zeuglodon). Bull. d. l'Acad. R. d. Belg., 2e sér., tom. XIl, pag. 22-28; 8". Bruxelles 1861.

7. InEx. Le Rorqual d'Ostende et les foulles d'Aneers. Bull. d. l'Acad. R. d. Belg., 2 $2^{\mathrm{e}}$ sér., tom. XII, pag. 453-483; 8․ Bruxelles 1861.

8. Ides. Le Rorqual du Cap de Bonne-Esperance et le Keporticati des Groenlandais. Bull. d. l'dead. R. d. Belg., $2^{\mathrm{e}}$ sér., tom. XVIII, pag. 389-400; 8. Bruxelles 1864.

9. Ideni. Note sur les Cétucés. Bull. d. l'dead. R. d. Belg., $2^{\circ}$ sér., tom. XX, pag. 851-854; $8^{\circ}$. Bruxelles 1865 .

10. IDEx. Recherches sur les ossements provenant du Crag d'Anvers. - Les Squatodons. Mém. d. l'Acad. R. d. Belg., tom. XXXV, pag. 1-85, pl. I-IV; $4^{\circ}$. Bruxelles 1865.

11. Inenr. Note sur un MLesoptodon Sovertiensis de 7a côte de Norwège. Bull. d. l'Acad. R. d. Belg., $2^{\text {e }}$ sér., tom. XXII, pag. 218-221; 8․ Bruxelles 1866 . 
12. Van Benedex P. J. Notice sur Ta découverte diun os de Bateine it Fumes. Bull.

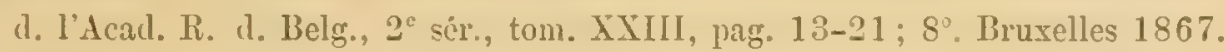

13. Inesr. Les Baleines et leur distribution géographique. Bull. d. l'Acad. R. d.

Belg., $2^{e}$ sér., tom. XXV, pag. 9-21, avec carte; 8․ Bruxelles 1868.

14. InEx. Les squelettes de Cétucés et les MLusées qui les ienferment. Bull. d.

l'Acar. R. đ. Belg., $2^{e}$ sér., tom. XXV, pag. 88-125; 8. Bruxelles 1868.

13. Inem. De la composition du bassin des Cétacés. Bull. d. l'Acad. R. d. Belg., $2^{e}$ sér., tom. XXV, pag. 428-433; 8. Bruxelles 1868.

16. Ident. La première côte des Cétacés, at mopos de la notice du Doct. J. E. Grar su" la distritution des Batcines. Bull. d. l'Acad. R. d. Belg., 2e sér., tom. XXVI, pag. 7-16, pl. I-II; 8. Bruxelles 1868.

17. Inex. Recherches sur les Squalodons - Supplement. Ném. d. l'doad. K. d. Belg., tom. XXXVIl, pag. 1-13, pl. I; $4^{\circ}$. Bruxelles 1868.

18. IDen. Totice sur les Batcinoptères du Nord de l'Atlantique. Bull. d. l'Acad. R. d. Belg., 2e sér., tom. XXVII, pag. 281-291, avec carte; $8^{\circ}$. Bruxelles 1869 .

19. Idex. Tote sur une Bulcinoptère cichouce dans l'Escunlt. Bull. d. l'Acad. R. d. Belg.; $2^{e}$ sér., tom. XXXVII, pag. 680-682; 8. Bruxelles 1869.

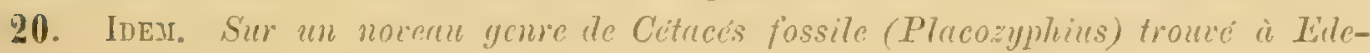
ghem. Mém. d. l'Acal. R. d. Belg., tom. XXXVII, pag. 1-13, pl. I-ll (le l'extrait); 4․ Bruxelles 1869.

21. IDEur. Les Cétacés, Teurs commensaux ct leurs purasites. Bull. 1. 1'Acad. R

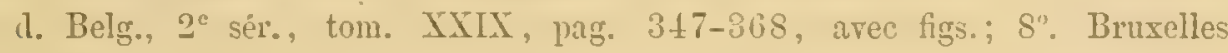
1870 .

22. IDEM. Observetions sur l'osteographic des Cétacés. Bull. d. l'Acad. I. d. Belg., $2^{*}$ sér., tom. XXX, pag. 380-388; 8. Bruxelles 1870.

23. IDEx. Recherches sur quetques poissons fossiles du Belgique (Encheizyphius brachyrthinchus). Bull. d. HAcad. R. d. Belg., 2e sér., tom. XXXI, pag. $493-$ 518 , arec 4 pls.; 8․ Bruxelles 1871.

24. IDEx. Communication verbale sur une Balcinoptère. Bull. d. 1'Acar. 1R. d. Belg., 2e sér., tom. XXXII, pag. t-כ̃; 8. Bruxelles 1871.

2:. Idear. Sur deux dessins de Cétacés du Cap de Bonne-Espérunce. Bull. d. l'Acad. R. d. Belg., $2^{\mathrm{e}}$ sér., tom. XXYVI, pag. 32-40, arec pl.; $8^{\circ}$. Bruxelles 1873.

21. Inex. Les Balcines de la Nouvelle Zélande. Bull. d. l'Acad. 1R. d. Belg., 2e sér., tom. XXXVII, pag. 382-387, arec pl.; 8. Bruxelles 1874.

27. Idex. Totice sur la grande Balcinoptère (B. Sibbaldii). Bull. d. l'Acal. R. d. Belg., 2e sér., tom. XXXIX, pag. 855-870, arec pl.; $8^{\circ}$. Bruxelles 1875 .

28. Iden. Los Pachyacunthus due Thuse te Fienne. Bull. d. I'Acad. R. d. Belg., $2^{\text {e }}$ sér., tom. XL, pag. 323-3ł0; 8". Bruxelles 1875 .

29. IDEx. Les ossements fossites du gemre Autocite, an IInsée de Linz. Bull." d. l'Acad. R. d. Belg., $2^{e}$ sér., tom. XL, pag. 537-5t9; $8^{\circ}$. Bruxelles 1875 . 
30. Van Beneden P. J. Le squelette de la Bateine fossite du Ifusé de Milan. Bull. d. l'Acad. R. d. Belg., $2^{\mathrm{e}}$ sér., tom. XL, pag. 736-758, avec pl.; $8^{\circ}$. Bruxelles 1875 .

31. Iden. Un môt sur la Baleine du Japon. Bull. d. l'Acad. R. d. Belg., 2ª́r., tom. XLI, pag. 28-37, avec pl.; 8 ${ }^{\circ}$. Bruxelles 1876.

32. IDEm. Les Thalassothériens de Battringen (Wïrttemberg). Bull. d. l'Acad. R. d. Belg., $2^{\mathbf{e}}$ sér., tom. XLI, pag. 471-495, avec pl.; 8. Bruxelles 1876.

33. Iden. Note sur Te Grampus grisens. Bull. d. l'Acad. R. d. Belg., $2^{\mathrm{e}}$ sẻr., tom. XLI, pag. 802-\$12, avec pl.; $8^{\circ}$. Bruxelles 1876 .

34. IDEM. Communication de deux lettres rélatives à des découvertes d'ossements fossiles en Italie. Bull. d. I'Acad. R. d. Belg., $2^{e}$ sér., tom. XLI, pag. 957$960: 8^{\circ}$. Bruxelles 1876.

33. Inenr. Le Rhachianectes glaucus des cótes de Catifornie. Bull. d. l'Acad. R. d. Belg., $2^{\text {e }}$ sẻr., tom. XLIII, pag. 92-96, arec pl.; 8. Bruxelles 1877.

36. IdEu. Sur les ossencuts fossiles des environs d'Anvers. Bull. d. l'Acad. R. d. Belg., $2^{e}$ sér., tom. XIIII, pag. 319-324; 8․ Bruxelles 1877.

37. IDEM. Un môt sur une Balcine capturéc dans la MLélitarrancée. Bull. d. l’Acad. R. l. Belg., $2^{\text {e }}$ sér., tom. XLIII, pag. 741-745; 8․ Bruxelles 1877.

38. Inen. Sur un Cachalot nain du Crag d'Anvers. Bull. d. l'Acad. R. d. Belg., $2^{\text {e }}$ sẻr., tom. XLIV, pag. 851-856, arec pl.; $8^{\circ}$. Bruxelles 1877.

39. Iden. Lu distribution géographique des Bateinoptères. Bull. d. l'Acad. R. d. Belg., $2^{e}$ se̊r., tom. XLV, pag. 167-178; 8. Bruxelles 1878.

40. Ions. La distribution géographique de quelques Cétodontes. Bull. d. l'Acad. R. d. Belg., $2^{\text {e }}$ sér., tom. XLV, pag. 401-409; 8. Bruxelles 1878.

41. IDEur. Un mót sur la pêche de la Balcine et les premières expéditions arctiques. Bull. d. l'Acad. R. d. Belg., $2^{e}$ sẻr., tom. XLVI, pag. 966-985; $8^{\circ}$. Bruxelles 1878.

42. Ides. Sur un envoi d'ossements fossiles de Cétacés de Croatie. Bull. d. l'Acad. R. d. Belg., $2^{e}$ sér., tom. XLTII, pag. 183-184; 8. Bruxelles 1879.

43. IDEM. Un môt sur quelques Cétacés échoués sur les côtes de la Míditerranéc et de l'Ouest de la Franee pendant le courant des années 1878 et 1879. Bull. d. l'Acad. R. d. Belg., $2^{\text {e }}$ sér., tom. XLIX, pag. 96-107; 8. Bruxelles 1880.

44. Iden. Balcine échoứe le 7 janvier 1880 sur les côtes de Charleston. Bull. d. l'Acad. R. d. Belg., $2^{\text {e }}$ sér, tom. XIIX, pag. 313-315; $8^{\circ}$. Bruxelles 1880 .

45. Idex. Un Hiperoodon capturé sur la grève d'Hillion en décembre 1880. Bull. d. l'Acad. R. «. Belg., $2^{\text {e }}$ sér., tom. L, pag. 9-11; 8. Bruxelles 1880 .

46. IDEM. Les Mysticètes à courts fanons des sables des environs d'Anvers. Bull. d. l'Acad. R. d. Belg., $2^{e}$ sér., tom. I, pag. 11-25; 8. Bruxelles 1880 . 
47. V. Beneden P. J. Description des ossements fossites des environs d'Anvers. Partie I. Amphithèriens, folio, pag. 1-88, pl. I-XVIII. - Partie II. Cétncés (Genres Balacna, Balacmela, Balaenotus), pag. 1-82, pl. I-XXXIX. Partie III. Cétucds (Genres Megaptera, Balaenoptera, Burtinopsis, Erpetocetus), pag. 1-88, pl. XL-CIX. Amnales du IIusée R. d. Hist. Nat. d. Belg., série Paléontologique, tomes, respect., I, 1877, IV, 1880, VIII, 1882. Bruxelles (ii suirre).

48. V. Bexenen P. J. et Gervais P. Osteographie des Cétacés vivants et fossiles; $4^{\circ}$, pag. 1-605. Atlas folio, pl. I-LXIV. Paris, Bertrand édit., 1880.

49. V. BENEDEN E. Sur la capture clans l'Escault au mois de novembre 1873 d'un Hyperoodon rostratum. Bull. d. l'Acad. R. d. Belg., 2e sér., tom. XXXVII, pag. 35 ; $8^{\circ}$. Bruxelles $187 t$.

:0. V. Banbecke. Quelques remurques sur les squelettes de Cétacés conservés à la collection d'anatomie comparée de l' Université de Gand. Bull. d. I'Acad. R. d. Belg., 2 $2^{e}$ sér., tom. XXVI, pag. 21-61, avec figs. (Rapport de M. P. J. V. Beneden sur ces Remarques, même vol., pag. $4-6) ; 8^{\circ}$. Bruxelles 1868.

31. Borson S. Mémoire sur quelques ossements fossiles trourés en Piémont. Mém. d. R. Acc. d. Sc. d. Torino, tomo XXXVI, pag. $33-48$, con 5 tav.; $4^{\circ}$. Torino 1831 (1830).

32. Braxim J. F. Untersuchungen ubber die fossiten und subfossilen Cetaceen Europre's, durch XXXIV Tafeln erläutert. Mem. d. l'Acad. Imp. d. Sc. d. St.-Pétersbourg, $6^{e}$ sêr., tom. $X X, n .1$, pag. 1-372; $4^{\circ}$. St.-Pétersbourg 1874.

33. IDEג. Ergnenzungen zu den fossilen Cetaceen Europa's, mit V Tafn. Mem. d. 1'Acarl. Imp. d. Sc. d. St.-Pétersbourg, $7^{\text {e }}$ sér., tom. XXI, n. 6, pag. 1-54; 4․ St.-Pétersbourg 1875.

34. VAN DEx Broech. Esquisse yéologique et puléntologique des dépôts pliocènes des environs d'Anvers. Ann. d. 1. Soc. Malacol. d. Belg., tom. IX, pag. 1-296, av. pls.; $8^{\circ}$. Bruxelles $1878(1876-78)$.

3̈:. Bruxo G. D. Illustrazione di un movo Cetacco fossile; $4^{\circ}$, pag. 1-20, tav. I-II. Mem. d. R. Acc. d. Sc. di Torino, ser. $2^{\text {a }}$, tom. I. Torino 1838.

36. Du-Bus G. Sur quelques IItamifères du Crag d'Anvers. Bull. d. L'Acad. R. d. Belg., $2^{\text {e }}$ sẻr., tom. XXIV, pag. 562-577; $8^{\circ}$. Bruxelles 1867.

37. IDEn. Sur différents Zyphioides nouveaux du Crag d'Anvers. Bull. d. l'Acad. R. d. Belg., 2 $2^{\mathrm{e}}$ sér., tom. XXV, pag. 621-630; 8. Bruxelles 1868.

38. IDEM. Tote sur une téconverte paléontologique faite it Boom. Bull. d. l'Acad. R. d. Belg., $2^{e}$ sér., tom. XIVI, pag. 20; 8․ Bruxelles 1868.

ä9. IDEu. MIammifères nouvenux du Crag d'Anvers. Bull. d. l'Acad. R. d. Belg., $2^{\text {e }}$ sér., tom. XXXlv, pag. 431-509; 8. Bruxelles 1872.

60. Capeltixi G. Balenotterce fossite nelle argille plioceniche di S. Lorenzo in collina, procincia di Bologna. - NorA - 16 ${ }^{\circ}$, pag. 1-11. Estr. d. Riv. Ital. d. Sc., Lett. ed Arti, n. 114 (2t nor.) e 115 ( $1^{\circ}$ dicemb.). Bo$\log 1121862$. 
61. Capelint G. Delfimi fossiti del Bolognese; 4, pag. 1-30, tav. I-III. Mem. d. Acc. d. Sc. d. Ist. d. Bologna, ser. 2a, tom. III. Bologna 1864 (1863).

62. Inex. Balenottere fossili del Bgolognese; $4^{\circ}$, tar. I-III. Mem. d. Acc. d. Sc. d. Ist. d. Bologna, ser. 2^, tom. IV, pag. 315-336. Bologna 1865.

1i:3. Iden. Sul Felsinoterio, Sirenoide haticoreforme dei depositi Tittorati pliocenici dell'untico bacino del MLediterranco e det MTar Nero; 4", pag. 1-49, tav. I-VIII. Mem. Acc. d. Sc. d. Ist. d. Bologna, ser. 3", tom. 1. Bologna 1872.

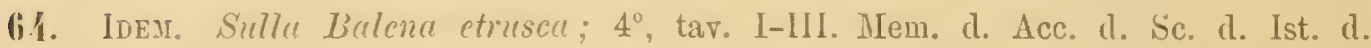
Bologna, ser. 3", tom. III, pag. 313-331. Bologna 1873.

(i:i. Iden. Sui Cetoterii bolognesi. - Considerazioni, 4º tav. I-II. MIem. d. Acc. d. Sc. d. Ist. d. Bologna, ser. $3^{2}$, tom. V, pag. 595-626. Bologna 1875 .

(ili. Inex. Sulle Balene fossili toscune; $4^{0}$, pag. 1-8. Att. d. K. Ace. d. Lincei, ser. $2^{2}$, tom. III. Roma 1876.

(i7. Idenr. Linomo priocenico in Toscana; $4^{\circ}$, pag. 1-17, tav. I-IV. Atti d. R. Acc. d. Lincei, ser. 2a, tom. III. loma 1876.

6is. Inens. Della Balena di T'aranto confrontata con quelle della Nuova Zelanta c con tahune fossili del Belgio e della Toscana. - Notizie; 4", pag. 1-34, tar. I-III. Mem. d. Acc. d. Sc. d. Ist. d. Bologna, ser. $3^{a}$, tom. VII. Bologna 1877.

(i9. InEs. Batenottere fossiti e Pachyacanthus dell'Itatia meridionate; 4․, pag. 122, tav. I-III. IIem. d. Cl. d. Sc. fis, mat. e nat. d. R. Acc. d. Lincei, ser. $3^{\text {a }}$, tom. I. Roma 1877.

70. Iden. Sulla Balenottera di MLondini. - Rorqual de la mer Adriatique di G. Curier; - 4", tar. I-IV. Mem. d. Acc. d. Sc. d. Ist. d. Bologua, ser. $3^{a}$, tom. VII, pag. 413-448. Bologna 1877.

71. Idex. Della « pietra Leccese » e di alcuni suoi fossili; $4^{\circ}$, pag. 1-34, tav. I-III. Mem. d. Acc. d. Sc. d. Ist. d. Bologna, ser. $3^{\text {a }}$, tom. IX. Bologna 1878.

72. Iden. Arañi di Squalotonte nella mollassa marnosa miocenica del Bolognese; $4^{\circ}$, pag. 1-2, con tar. Mem. d. Acc. d. Sc. d. Ist. d. Bologna, ser $4^{a}$, tom. II. Bologna 1881.

73. Inenr. Del Tursiops Cortesii e del Delfino fossile di IIombercelli nell'Astigiana: $4^{\circ}$, pag. 1-12, con tar. Hem. d. Acc. d. Sc. d. Ist. d. Bologna, ser. $4^{\text {a }}$, tom. III. Bologna 1882.

74. Iden. Di un'Orea fossile, scoperta a Cetome in Toscana; $4^{\circ}$, pag. 1-25, tar. (doppie) I-IV. Mem. d. Acc. d. Sc. d. Ist. d. Bologna, ser. $4^{\text {a }}$, tom. IV. Bologna 1883.

73. Carus und Gerstaeoker. Handunch der Zoologie. I Bd., $1^{\text {sle }}$ Haelft, S. 163$172 ; 8^{\circ}$. Leipzig, Engelmann, 1868.

76. Cocchi I. Linomo fossile nell'Italia centrale. - Studi paleontologici; $4^{\circ} \mathrm{gr}$, pag. 1-80, tar. I-IV. Mem. d. Soc. Ital. d. Sc. Nat. d. Milano, rol. II, n. VII. Milano 1867. 
77. Connala E. Fauna d'Itatia. Parte 1. Catalogo descrittivo dei Mammiferi osservati fino ad ora in Italia; $12^{\circ}$, pag. 1-80. Milano, Vallardi (con carta della distribuzione geografica dei Cetacei in Italia).

78. Contesi G. Sugli scheletri di un Rinoceronte africano e di una Balena ed altre ossa di grandi quadrupedi e cetacei dissotterrate nei Colli piacentini; 4", pag. 1-26, tav. I-III. Milano, Marelli stamp., 1808.

79. IdeM. Saggi geologici degli Stuti di Parma e Piacenza; 4?, pag. 1.166, tav. I-VII. Piacenza, torchi del Majno, 1819.

SO. Cuvier G. Recherches sur les ossements fossiles (4 édit., texte $8^{\circ}$, plls. $4^{\circ}$ ), texte tom. VIII, $2^{e}$ part. Atlas pl. 220-228. Paris 1836.

81. Gastaldi B. Cemi sui Vertelrati fossili del Piemonte; $4^{\circ}$, pag. 1-68 (tar. I-X). Mem. d. R. Acc. d. Sc. d. Torino, ser. 2 ${ }^{2}$, tom. XIX. Torino 1858.

82. IDEM. Antracoterio di Agnana, Balenottera di Ca-Tunga presso S. Damiano, e Mastodonte di MIongrosso. Lettera; 80, pag. 1-4. Atti d. Soc. Ital. d. Sc. Nat., rol. T. Mlilano 1863.

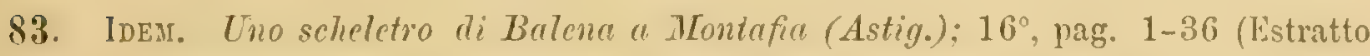
dalla Gazzetta Picmontese). Torino, Tip. C. Favale, 1875.

8.1. Gaudry A. Les enchainements du monde animal dans les temps géologiques. - Mammifères terticires; pag. 29-40, I vol., $8^{\circ}$, av. gravs. Paris, Savy éd., 1878.

83. Gervals P. Zoologie et Paléontologie francaise; $4^{\circ}, 2^{e}$ édit. Paris, Sary éd., 1859, pag. 276-320, atlas passim.

86. Idenr. Lettre sur Tes Squalodons. Bull. d. l’Acad. R. d. Belg., $2^{e}$ sér., tom. XIII, pag. 462-469, arec pl.; 8 ${ }^{\circ}$. Bruxelles 1862.

87. Iden. Sur la Bateine de Tu Mćditerranée, Rorqualus antiquorum. Bull. d.

l'Acad. R. d. Belg., $2^{e}$ sér., tom. XIV, pag. 186-192; 8. Bruxelles 1862.

88. Idey. Coup d'ccit sur Tes Mammifères fossiles de l'Itutic. Bull. d. 1. Soc.

Géol. d. France, $2^{e}$ sér., tom. XXIX, pag. 92-103; 8. Paris 1872.

89. Gieber C. G. Fanna der Ironwelt mit steter beriichsichtignng der lebenden Thicic. $1^{\text {ster }}$ Bd., $1^{\text {sle }}$ Abth., S. 225.239; S $^{\circ}$. Leipzig, Brochhaus, 1847.

90. Gray J. E. Catalogue of the specimens of Mammatia in the collection of the British

Muscum. Part I, Cetaced; pag. 1-153, pl. I-VIII; 16 . London 1850.

91. IDEM. Catalogue of Seals and Whates in the British MInseum; $2^{\mathrm{d}}$ édit., pag. 1-402, with woodets; $8^{\circ}$. London 1865.

92. Idex. Supplement to the Catalogue of Seals and Whates in the British MIuseum; pag. 1-103, with woodcts; $8^{\circ}$. London 1871.

93. IDEn. Synnpsis of the specics of Whates and Dotphins in the collection of the British MLuscum; pag. 1-10, pl. I-XXXVII; 4. London 1868.

91. GuÉRIN R. Etudes zoologiques et paléontologiques sur la femille des Cétacés. Thése; pag. 1-146, arec 3 cartes; $12^{\circ}$. Paris 1874.

9:3. Lawley R. Nrovi studi soma ai Pesci ed altri Vertebrati fossili dolle colline Toscane. Firenze 1876.

96. IDEM. Resti di Felsinotherium Forestii Cap. tronati presso Voltera. Atti d. Soc. Tosc. d. Sc. Nat., vol. III, fasc. II; 8", pag. 341. Pisa 1878.

3. A. Portss. 
97. Von MIEYEer H. Dolphinus acutidens aus der MLolasse bei Stockach. Palaeontographica. Bd. VII., S. $105-109$, Taf. XIII.; 4․ Cassel 1860.

98. Movron M. Sur le gisement un Cachalot nain (Physetemla Dubusi). Bull. d. $1^{\prime}$ Acad. R. d. Belg., $2^{e}$ sér., tom. XIV, pag. 178-182; 8. Bruxelles 1878.

99. Pictet F. J. Traité de Patcontologie. Paris 1853-57. Texte $16^{\circ}$, tom. I, pag. 368-388. Atlas 4", pl. XIX. Paris, Sary, 1857.

100. Van Raemoxor. Sur lu découverte d'ossements fossiles ì Saint-Nicolas. Lettre communiquée par M. Sinet. Bull. d. l'Acad. R. d. Belg., 2e sér., tom. VIII, pag. 197-199; $8^{\circ}$. Bruxelles 1859.

101. Scarabelis L. Di una Batena, di un Delfino e di molte conchiglie cerate dai Colti del Piacentino per operu del sig. PoDesti; pag: $1-11 ; 12^{\circ}$.

102. Strobel P. Notizie preliminari su le Balenoptere fossiti subapennine del II useo Parmense. Boll. d. K. Com. Geol. d'Italia, rol. VI, n. 5-v, pagine $131-140 ; 8^{\circ}$. Roma 1875 .

103. IdEu. Iconografia comperate delle ossa fossili del Gubinetto di Storia Naturale dell'Universiti di Parma. Fasc. I, DaTenotteride (Cetoterio e IIegettera); $4^{0}$ gr., pag. 1-32, tar. I-V. Parma, Battei ed., 1881.

104. De-Zigno A. Sirenii fossili trorati nel Veneto, pag. 1-30, tav. I-V. Mem. d. 1. Ist. Ven. d. Sc., Lett. ell Arti, vol. XVIII; $4^{\circ}$ gr. Venezia 1875.

103. IDEM. Sopma $i$ resti di uno Squuladonte scoperti nell'urcnaria miocena del Bellunese; pag. 1-19, tar. 1, fol. Mem. d. R. Ist. Ven d. Sc., Lett. ed Arti, vol. XX. Venezia $1876,4^{\circ} \mathrm{gr}$.

106. Iven. Sopre un moro Sirenio fossite scoperto nelle colline di Bre in Picmonte; pag. 1-13, tav. I-VI. Mem. d. Cl. d. Sc. fis,, mat. e nat. d. R. Acc. d. Lincei, ser. $3^{a}$, tom. II; $4^{n}$. Roma 1878.

107. InEx. Nuove osservazioni sull'Hatitherium veronense Zigno; pag. 1-8, tav. I. Mem. d. R. Ist. Ven. d. Sc., Lett. ed Arti, rol, XXI; $4^{0}$ gl. Venezia 1880.

108. Zitter C. A. Ueber Squalonon Zariensis uns Niederbayern; S. 1-46, mit. Taf. Sep. Abdr. aus d. $24^{\text {te }}$ Ber. d. Naturhist. Vereins Augsburg; $8^{\circ} .1878$.

109. Pontis A. Nuovi studi sulle traccic attribuite all'nomo plioccnico; $4^{\circ}$, pag. 1-30, tar. I-II. Wem. d. R. Acc. d. Sc. di Torino, ser. $2^{a}$, tom. XXXV. Torino 1883 (1).

110. Capeltixi G. Del Zifoide fossile (Choneziphius planirostris) scoperto nelle sablic plioceniche di Fungonero presso Siena. Mem. d. Cl. d. Sc. fis., mat. e nat. d. $R$. Acc. d. Lincei, ser. $4^{\text {a }}$, rol. I; $4^{\circ}$, pag. 18-29, con tav. doppia. Roma 1885 (1884) (2).

111. Idexr. Resti fossili di Dioplodon e Mesoplodon. Mem. d. Acc. d. Sc. d. Istit. d. Bologna, ser. IV, tom. IV; $4^{\circ}$, pag. 291-306, con tav. doppia. Bologna 1885 (2).

(1) Lavoro eseguito contemporaneamente al presente.

(2) Lavori pubblicati dopo la chiusura del presente, ma consultati prima di intraprenderne la stampa. 


\section{PA RE P IR I I A}

\section{MISTIGITI}

\section{BALENIDI}

\section{GENERE BALAENULA.}

N. 1. Balaenula sp.

Fla, 1-?

Il gencre Butacmula, che cosi copiosi aranzi di sè lasciò nel bacino di Anversa, venne già da tempo riconosciuto pure in Italia. Il l'rof. Capellini raccolse nel Museo di Bologna gli aranzi delle Balenule toscane, e frammezzo ai copiosi avanzi di Cetacei fossili che andarono man mano scoprendosi nell'Astigiana ed accumulandosi nel Museo di Torino, il Prof. V. Beneden passando per questa cittì riconoblue doversi pure trovare ossa attribuibili al genere Balacmula.

Dopo la visita dello illustre C'etologo belga alle nostre collezioni, il Museo di Torino non ha piu riceruto alcuno aranzo riferibile al genere suddetto, per cui tutto ciò che gli spetta con sicurezza si compendia per ora in un solo ed unico aranzo. Tale è la settima vertebra cervicale che lo fatto disegnare (fig. 1) dalla faccia anteriore e (fig. 2) veduta di fianco.

Questa vertebra, avente il cliametro antero-posteriore di $59 \mathrm{~mm}$, il trasversale di $126 \mathrm{~mm}$. ed il verticale di $95 \mathrm{~mm}$, appartenne ad un mimale gia adulto e nel quale ogni traccia della primitiva separazione delle epifisi exa gia scomparsa e l'osso compattissimo alla periferia ḋ molto più spugnoso allo interno.

La faccia articolare anteriore, piì larga che alta, col margine superiore rettilineo, ed i laterali e l'inferiore arrotondati, si presenta leggermente convessa, specialmente al centro, dove per mezzo di una superficie abbastanza irregolare e rugosa presentante un cinque centimetri di maggior diametro tendeva ad unirsi colla faccia posteriore della vertebra precedente. Anzi verso il centro di questa faccetta di unione si eleva ancora di un 4 o 5 millimetri al davanti di essa un tubercolo tondeggiante di un centimetro di diametro e composto di materia ossea estremamente compatta, il quale evidentemente era gia saldato colla vertelsra precedente e dalla quale venne poi rotto posteriormente alla morte dello animale essendo esso troppo poco resistente in proporzione col gran peso e colla grande superficie ancor libera delle vertebre $6^{2}$ e $7^{2}$ ed esposta a pressioni non uniformi durante la fossilizzazione.

La faccia posteriore di questa stessa rertebra è meno rettangolare arendo gli ongoli più smussati ed il margine superiore non più rettilineo. Essa è pianeggiante 
nella parte periferica, lievemente incavata nella centrale. Il centro poi è marcato da minuta impressione di $3 \mathrm{~mm}$. circa di diametro e di $1 \mathrm{~mm}$. di profondità e che appare come cagionata dalla presenza di un tubercolo minuto, analogo a quello che questa vertebra presenta sulla sua faccia anteriore, avente esistito ancora sulla faccia anteriore della prima dorsale, ma che abbia avuto un molto minore sviluppo.

La vertebra cervicale che è ora l'oggetto della nostra descrizione trovasi, come si può ben scorgere dalle figure, ridotta al solo corpo, mancando l'arco neurale e non essendo visibili che $\mathrm{i}$ punti d'inserzione dei due pedicoli di esso. Rilevasi però come il canale neurale fosse eccessivamente largo ed aresse un fondo piano nel mezzo ed incurvato ai lati, e come l'arco neurale dovesse da lungo tempo esser saldato al corpo allorchè l'animale venne a morte.

A differenza di ciò che osservasi nelle Balenule di Anversa, questa settima cervicale presenta ancora, quali traccie delle apofisi trasverse inferiori, dei grossi tubercoli di sostanza ossea spugnosa, el a meta della faccia inferiore, un altro tubercolo a mo' di carena che può esser tenuto come ultimo rappresentante del Tallone che, molto piu sviluppato, arremmo doruto scorgere sotto alle rertebre cerricali precedenti.

In complesso: la forma el i caratteri riconoscibili su questo corpo di vertebra ci fan ben presto conoscere come essa abbia doruto appartenere ad una specie del genere Bälacmula. La presenza però di rappresentanti dei processi trasversi inferiori e di una traccia di Tallone ci farebbero ben supporre che la specie che lasciò questo avanzo nelle sabbie dell'Astigiana dorette essere diversa da quella che li lasciò nel bacino di Ansersa. Aruto però riguardo alla somma variabilitì dei caratteri di tal ordine, osservabile in individui della stessa specie di Misticeti, ed al fatto che il nostro pezzo non è sufficiente per fornire tale quantità di caratteri da far riconoscere in arvenire la specie piemontese da altre trovate o trovabili, mi limito a registrare col nome di Bulacmula sp. l'unico avanzo che rivela come anche in Piemonte presso al volgere dell'epoca terziaria vissero Misticeti del genere Balaemula che non la cedevano per dimensioni a quelli cle vissero nelle regioni settentrionali d'Europa.

Questa rertebra fu raccolta dal Prof. Gastaldi nelle sabbie gialle dell'Astigiana.

Il valente Cetologo di Louvain attribuisce al genere Balacmla, invece del pezzo aranti descritto, una sinostosi cervicale trovata da più lungo tempo nell'Astigiana e facente parte della collezione del R. Inseo di Hineralogia e Geologia, di cui dice: " des rertébres malades de Balaenula qui sont soudées entre elles ». Dopo avere a lungo esuminata e studiata tale sinostosi, ho creduto bene di attribuirla, anzichè ad un Balenide, ad un Cetaceo a denti e l'ho collocata in capo ad essi nella famiglia dei Fiseteridi descrivendola col nome di Priscophyseter tipus.

I principali motivi che mi indussero a non lasciare fra $i$ Balenidi un tale pezzo si possono cosi brevemente riassumere: la sinostosi in questione appartenendo alla regione cervicale posteriore, come lo dimostrano e la posizione dei pedicoli dell' arco neurale e l'inclinazione del fondo del canale neurale, il corpo delle vertebre non presenterebbe più alcuna analogia con quello a contorno quasi quadrangolare delle vertebre omologhe delle Balenule e dei Balacnotus o con quello meno allargato delle Balenottere; le apofisi trasverse inferiori che sono ancora presenti od accemate nelle ultime vertebre dei Cetacei a fanoni, mancano affatto a questa sinostosi e ciò analogamente a ciò che osserviamo nei Fiseteridi. 


\section{BALENO'TTERIDI}

\section{GENERE BALAENOPTERA.}

\section{N. 2. Balaenoptera Gastaldii (Strobel).}

Fเศ. 3-33.

\section{LA BALENOTTERA DI CORTANDONE}

Cetotherium (Cetotheriophanes?) Cortesii Brand (non Desm.). Unters. ucb. d. foss. u. subf. Cetacecn Europa's, pag. 153, 1873.

Plesiocetus Cortesii Desm. Van Beneden, Lu buteine fossile du Musée de Milan.

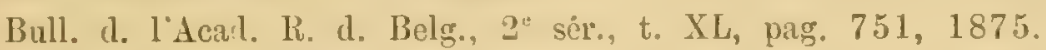

Cetotherium Gastaldii Strober. Not. prel. su le Balenopt. foss. subapenn. d. Mus. parmense. Boll. d. R. Com. Geol. d'Italia, vol. VI, pag. 136, 1875.

Cetotherium Cortesii var. Gastaldii Strober. Icon. comp. delle ossa foss. d. Gubin. di St. Nat. dell'Univ. di P'urmu, pag. 8 e seg., 1881.

Nell' anno 1862 il Prof. Gastaldi scavava in territorio di Cortandone gli avanzi di un piccolo Cetaceo che alcuni anni dopo faceva disegnare, inviando le figure al Prof. Brandt di Pietroburgo. Questi ne dava la determinazione e la descrizione a pag. 153 e seg. della sua classica opera: Ricerche sopra $i$ Cetacei fossili e subfossiti d'Europa, comparsa nel 1873. Come arrò più volte occasione di fare nel corso di questo mio lavoro, riporto qui, traducendolo, tutto il brano del Brandt, che si riferisce a questo caratteristico fossile, riservandomi di aggiungervi in seguito quanto crederò possa io aver ancora osservato di interessante per la esatta determinazione e conoscenza dello animale di cui è questione.

Ecco adunque il capitolo del Brandt:

«Spec. 13. Cetotherium (Cetotheriophanes?) (1) CoRTEsil Nob., tar. XXI e Xxir.

«Balcine de Cortesi; Desuoulins, Dictionn. class. d'Hist.nat., t. II, pag. 165. - Rorqual de Cortesi; Bortard, Dictionn. univ. d'Hist.nat., p. D'Orbigny, t. II, pag. 443. - Bulcine du sous-genre des Rorquals Cuvier, Rech., édit. 8º, t. VIII, p. 2, pag. 314. Autre squelette. - Rorqualus Cortesii (Balnenu Cortesii) DEs-

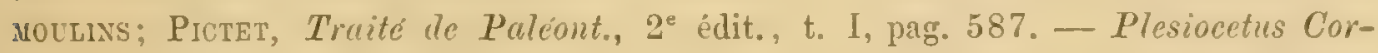
tesii; Vax Beseden, Ostéographie e. p. - Plesiocetus Cortesii; P. Gervals, Bull. de la Soc. Géol. de France, $2^{e}$ sér., t. XXIX, p. 100 (Gli aranzi del medesimo trovantisi nel Museo di Torino).

(1) " Col punto di interrogazione deve essere accennato che il Cetotherium Cortesii per la forma non ancora conosciuta della scapola non può venir aucora riportato con piena sicurezza nel soltogenere Cetotheriophanes; ciò che si può dire ancora dei Cetotherium Capellinii e Vandellii \# (Nota esistente nel testo del BrandT). 
"Pello stabilimento affatto esatto del Cetotherium Cortesii si dovrebbe senza dubbio prender le mosse da precise ricerche sullo scheletro scoperto nel 1816 dal Cortesi e clıe egli (nel 1819) per sventura non descrisse sufticientemente e non figurò pershè nella determinazione della specie lo ritenne per identico a quello da lui prima scoperto, nella quale ammissione fu seguito dal Cuvier. Desmoulins fece, come sopra fu indicato, la fortunata proposta (secondo me), sulla sola base di alcune differenze, per certo assai salienti, nella lunghezza lel cranio e del corpo, segnalate da Cortesi e da Cuvier, per le quali lo scheletro in questione si distingueva da quello scoperto rel 1806 dal Cortesi, di creare una nuova specie di Bulacua, la Balcine de Cortesi, mentre egli, come già redemmo, attribui ad una Balcine de Curier lo scheletro scoperto dal Cortesi nel 1806. Boitard segui Desmoulins, registrỏ però le due specie come Balacnoptera Curioni e Cortesii.

«Poichè dcllo scheletro trovato dal Cortesi nel 1816 [il quale dopo ciò non si sa dove sia passato (1)] non ci troviamo ad aver dinanzi alcuna descrizione o figura, cosi l'ammettere che esso appartenesse ad una specie particolare non si fondara che su differenze di lunghezza del cranio e del corpo, le quali, come è affatto maturale, non bastano per se stesse in alcun modo a far riguardare la Balcine de Cortesi qual tipo di una specie propria, dirersa dalla Buleine dr Cuxier. Le opinioni dei Paleontologi sopra la esistenza delle or nominate due specie sono per conseguenza divise. Pictet le riconosce, Giebel (Dic Süurgethiere, p. 86) ritiene la loro indipendenza ancor come dublia. Canellini, che arera reduto lo scheletro di Milano, riporta gli aranzi appartencnti alla sua specie bolognese (per quanto a me pare ma specie a parte $\left[C_{r}-\right.$ tothcrimu Capsellinii mh.]) dubitativamente al un Rorqualus Cortesii. Van Beneden (Ostcogr., p. 242) dichiara che tutti gli aramzi italiani scoperti la Bianconi (scrivi Blanconi), Cortesi e Capellini appartengono ad una sola specie di Butuenopteru. Sotto questo nome di Baluenoptera intende egli però senza dubbio il suo Plesiocctus Cortesii (ibirl, pag. 288), del quale però sino ad ora non forni alcuna completa descrizione e sinonimia motirata. P. Gerrais (loc. cit.) è di opinione che tutti gli avanzi di Cetucei "fanoni conservati in Torino, Bologna e Napoli appartennero al Plesincetus Cortesii.

"Quantungue io non abbia avuta occasione di studiare lo scheletro su cui fu stabilita la Bulcine de Cortesi di Desmoulins, con tutto ciò per mezzo dei bei disegni dei resti più interessanti di Cetoterii conservati nei MIusei di Torino, che io devo alla grande bontà del sig. Prof. Gastaldi, come delle eccellenti figure, fatte di propria mano, delle parti della Bulcine de Curier del Mtuseo di Milano, liberalnente partecipatemi dal sig. Prof. Cornalia, sono stato messo in caso di distinguere con sicurezza una seconta specie italiana di Cetotherium che viene documentata dagli avanzi di Torino e che, specialmente per riguardo alle sue dimensioni, siccome dimostreremo più a lungo, può essere ritenuta per quella della Batcine de Cortesi di Desmoulins.

1) Vedremo in seguito come tale scheletro sia poi ricomparso alla luce, trovandosi ora nel R. Museo di Parma, dove, per cura del Prof. Strobel, venne completamente liberato dalla roccia che ancora lo avvolgeva e da lui descritto, figurato e paragonato coi jiù classici avanzi di Balenottere scoperte nell'Italia superiore (Nota estranea alla citazione). 
« Gli avanzi di Torino, per quanto li conosco dalle figure, consistono in un cranio quasi completo, in vertebre diversissime, molte coste, un omero ed un'ulna (1). I disegni del Gastaldi comparati con quelli del Cornalia Iel Cetotherium Curiori ci dimostrano chiaramente le seguenti differenze:

" La porzione occipitale più estesa nel cranio di Torino (tar. XXI, fig. 1-5) non è assottigliata posteriormente. La squama occipitale è più corta che larga e non appare rigonfia ai lati. La sutura lambioidea lell'occipite appare non ripiegata e non assomigliante quindi a quella dei Cetotherium Iiuthliei, Helmersenii e Trantellii. Le squame temporali appaiono più rigonfie. Le fosse tomporali sono più corte e men larghe che pel Crtotherium Cuvieri. I processi gingali dei temporali, piì grossi e piu corti, si aploggiano ai processi orbitali, a quanto pare, più rigonfii e piu piccoli dei frontali. II bordo anteriore di questi ultimi appare, specialmente nella sua parte interna, meno arcato allo avanti. I processi orbitali dei mascellari sono meno rigonfii nella parte posteriore, urenti nella anteriore. La porzione frontale e parietale lel cranio è più larga e piu corta che pel Cetotherium Curicri e rigonfia ai lati. La parte articolare della mandibola pare si comporti nell' essenziale come pel Cetotherizm Curieri.

« La lunghezza del cranio deve, secondo le dimensioni delle quasi complete mandibole, essere calcolabile a circa 1,22 m. La lunghezza della mandibola tocca $1,10 \mathrm{~m}$. La larghezza dell'occipite 0,62 m. L'atlante (ibid. f fig. 7-10) appar piu stretto ed alquanto piu alto. L'Epistrofeo (tar. XXII, fig. 11-14) ì piir alto e possiede un processo spinoso molto consid revole nonchè due processi trasversi (per parte) completamente separati, dei quali il più alto molto corto, l'inferiore molto più grande, molto lungo, appare alla estremitì allargato e qualcho poco rivoltu allo ingiù. Le altre vertebre cervicali come pure le dorsali anteriori (2) (ibid., fig, 15-16), paino possedere brevi corpi. Le vertebre lombari (ibid., fig. 17-23 e tav. XX1, fig. 6 (3)) sono più corte e meno svelte. Lo stesso dicasi delle vertebre caudali (tav. TXII, fig. $24-27$ ).

« Le forti coste (tav. XXII, fig. 30-31) mostrano il carattere delle coste di Cetoterio.

(1) Giova qui notare come di vertebre appartenenti allo stesso individuo non ve ne siano che otto fra cui le tre prime cervicali, una lombare o quattro caudali; tutte le altre vertebre qui menzinnate el anche da altri autori confuse con qreste, spettano al un altro individuo, di cui il Prcfessore GAstal.DI scavò a Ca-lunga numerose vertobre cho io avrò a ricordare in seguito riferendole alla. specie pii comune iu Piemonte: il Plesiocetus Cortesii V. Be.s. Similmente dello individuo di Cortandone, oggetto della presente descricione, non si trovarono che due sole coste. Per contro dipo avere, per rettificarla, sottratte molte vertelre e molto coste dalla lista del Braxor, occorre aggiungere che assieme al cranio vennero trovate le due manlibole conservatissime, che, secondo l'appunto mossogli da altri autori, vennero dal Brand prese pel prino paio di coste; inoltre un osso del bacino, che il BRAXDT ricoldu e figura più sotto, un frammento di osso joide, che passò finora inosservato, e sei fra ossa metacarpali e falangi (Nota estr. a. cit.).

(2) Di questo individus non venne, come gia annunciai, trovała alcuna vertebra dorsale. Le figure 10̄-l:j della tavola XXII del BRANDT rappresentano la terza vertebra cervicalo (Nota estr.a. cit.).

(3) Di vertebre lombari spettanti a questo individuo non ve u'ha che una, quella figurata figura 17-20 della tav. XXII! Le altre che qui menziona il BRANDT e che egli raffigura (tav. XXI, figura 6) spettano allo individuo di Ca-lunga di cui parleremo in seguito registrandolo fra i Cetoterii piemontesi apparteneuti alla vera Balaenoptera Cortesii (Nota estr. a. cit.). 
"La condizione di lunghezza dell'omero, di forma analoga a quello del Cetotherium Cuvieri (ibid., fig. 33), a quella dell'avambraccio si mantenera pure in generale come pel Cetotherium Curieri; contuttocio pel Cetotherium Cortesii le ossa del braccio poterono essere alquanto più corte.

"L'olecrano del cubito, che è alquanto più piegato (ibid., fig. 28), ci mostra un borlo posteriore arcato.

« Mancano disegni della scapola, delle ossa carpali e metacarpali, delle falangi.

«Se la lunghezza, che veniamo di calcolare a m. 1,22, del cranio or or descritto si comporta nello essenziale proporzionalmente come pel Cetotherium Curieri (ciò che si può in generale ammettere) allora la lunghezza dello scheletro cui appartenne questo avanzo torinese si potrebbe calcolare a m. 4,27. Poichè lo scheletro scoperto dal Cortesi nel 1816, riferito dal Desmoulins ad una specie Baleine de Cortesi secondo i dati di Cuvier, tolti dal Cortesi, possedera una lunghezza di 12 piedi e 5 pollici $(=4,03 \mathrm{~m}$.) ed una lunghezza del cranio di 4 piedi $(=1,17 \mathrm{~m}$.), cosi ne risulta tra quello e gli avanzi di Torino, specialmente quelli cui appartiene il cranio figurato, per rapporto alle dimensioni del corpo una tale coincilenza quale si trova con lievi modificazioni presso i diversi individui di una stessa specie. Se per conseguenza anche quello individuo torinese a cui appartenne il cranio era alcunchè più grande di quel di Cortesi, non può questo fatto dare alcun argomento contro all'unità specifica.

"Si può per conseguenza annoverare una parte, se anche possibilmente non tutti, degli avanzi di Torino fra i più che probabili tipi del vero Cetotherium Cortesii (=Balcine de Cortesi Desuroumixs); una ipotesi la di cui diretta conferma per mezzo del ritrovamento degli avanzi dello scheletro scoperto da Cortesi nel 1816 sarì ognora 111 giusto desiderio (1). Possa riescire al Prof. Cornalia di trovare gli ora nominati aranzi in uno dei MLusei d'Italia, di compararli precisamente con quelli conservati in Torino ed appartenenti a diversi individui di Cetoterii e di descriverli per disteso!

« (quali località di provenienza dei numerosi avanzi che si trovano nel Museo di Torino vengono date Cortandone e S. Lorenzo. Si trovano però fra essi anche le 36 (2) vertebre e le coste scoperte dal Gastaldi (Atti della Soc. Ita7. d. Sc. Nat., 1862, IV, p. 88. - Rerue Sc. Ita7., 1862, pag. 40) (Ved. tar. XXI, fig. 6), l'ultimo novembre 1862 , presso la stazione di S. Damiano alla.Ca-lunga nell'argilla azzurra pliocenica inferiore ».

Ben poco tempo dopo che il Brandt conchiudeva la sua descrizione degli avanzi torinesi collo augurio che il Cornalia riescisse a trovare lo scheletro 1816 del Cortesi, il suo desiderio veniva pienamente soddisfatto, se non dal Cornalia, da altri. Infatti

(1) Due anni dopo il Prof. Strobel, annunziando come si trovasse in Parma lo scheletro 1816 di Cortesi, emetteva l'opinione che esso fosse a considerarsi bensì come diverso specificamente da quello trovato nel 1806, conservato a Milano, a cbe per conseguenza gli toccasse di venir tenuto come il tipo della specie Baleine de Cortesi Dess. = Balaenoptera Cortesii Borr., ma che esso differisse ancora dallo scheletro 1802 scavato dal Gastaldi a Cortandone e che questo per conseguenza dovesse venir nuovamente descritto e distinto con un nuovo nome, ciò che poi fece egli stesso nella Iconografia del 1881, come vedremo ben tosto (Nota estr. a. cit.).

(2) Probabilmente è qui sfuggito un errore di stampa nel testo terlesco, le vertebre di Ca-lunga invece dí 36 non essendo che 26 (Nota estr. a. cit.). 
nel 1875 lo Strobel pubblicara nel rol. VI del Bollettino del Comitato Geologico Italiano le sue notizie preliminari sulle Balenottere fossili subapennine del Museo parmense e ne dara una succinta descrizione accennando ai caratteri per cui tale scheletro si distingueva, secondo lo Strobel stesso, e dalla schcletro 1806 del Cortesi e dagli altri schcletri del IIuseo parmense e da quello scoperto nel 1862 dal Gastaldi a Cortandone. Ne reniva di naturale conseguenza che dovendosi considerare come una specie a parte lo scheletro Cortesi 1816, a quello toccasse l'appellativo Cetotherium Cortesii e che lo scheletro 1862 di Cortandone dovesse riceverne un nuovo. Lo Strobel proponeva quindi pello scheletro di Cortandone il nome di Cetotherium Grstaldii.

Occorre però notare come nella seduta 4 dicembre 1875 il Van Beneden presentava alla R. Accademia del Belgio le sue considerazioni cul i suoi studi sullo scheletro della Baleine de IIilan. In questo lavoro egli dà il risultato degli studi che arera fatti in Italia nel 1874 dopo aver comparato il materiale di Milano, di Bologna e di Torino e risti i disegni e gli appunti fatti dallo Strobel sul materiale di Parma.

Come conclusione delle comparazioni fatte, il Van Beneden manifesta la sua opinione, secondo la quale lo scheletro 1806 Cortesi, lo scheletro 1816 Cortesi, lo scheletro Capellini, lo scheletro 1862 Gastaldi e gli scheletri $3^{\circ}$ Cortesi e Podesta del IIuseo di Parma appartengono tutti ad una sola e medesima specie, per la quale deve ritenersi il nome di chi primo ne seppe interpretare lo analogie, e chiamarla Plesiocetus Cortesii. Tale opinione riespone il Van Beneden nel suo classico lavoro: Description des ossements fossiles des environs d'Aners, $3^{e}$ Partie, edita presso al volgere rel passato 1882 .

E finalmente nella prima metì del 1881 lo Strobel pubblicava la sua Iconografiu delle ossu fossili del Gabinetto de Storia Naturale della Universitì ni Parma, nella quale esplicando le idee emesse nel 1875 , cercava di dimostrare come tutti i Cetoterii scoperti nell'Alta Italia dovessero venir riuniti in una sola specie, il Plesiocetus Cortesii e come questa dovesse comprendere 4 varietì, cioè: $1^{\circ}$ il Cetothcrium Cuvicri, rappresentato dallo scheletro $1806^{2}$ Cortesi o del Museo di Milano e dallo scheletro giovane $3^{\circ}$ Cortesi del Nuseo $1 i$ Parma; $2^{\circ}$ il C. Cortesii, rappresentato dallo scheletro 1816 Cortesi o del Museo di Parma; $3^{\circ}$ il C. Capellinii, rappresentato dallo scheletro Capellini o del Mruseo di Bologna e da quello del Podestì del MIuseo di Parma; $4^{\circ}$ il C. Gastuldii, rappresentato dallo scheletro $180^{\circ}$ Gastaldi di Cortandone o del Mruseo di Torino.

Se dopo ciò anche a me fia lecito l'esporre una propria opinione, dirò che quantunque la descrizione del Brandt sia incompleta, come fatta soltanto in base a disegni a proporzioni ridotto, pur tuttavia mi associo a lui nel considerare lo scheletro 1862 di Cortandone, scoperto dal Gastaldi, come tipo di una muora specie distinta da quelle che vennero finora scoperte in altri punti dell'Alta Italia, e nel ritenerla per conseguenza degna di ricevere un nome specifico a sè. Il nome da applicarle, non potendo essere quello proposto dal Braudt stesso, perchè già anteriormente impegnato per un'altra specie, ci viene suggerito dallo Strobel il quale, pure inchinandosi all'autorità del V. Beneden nello ammettere come appartenenti a specie unica tutti i Cetoterii dell'Alta Italia, non può a meno di dare importanza ai caratteri del teschio di Cor-

4. A. PORTIS. 
tandone facentone almeno una varieti che dedica allo scopritore chiamandolia Cetotherium Gastathii. A noi non resta che di elevare al rango di nome specifico il nome distintivo di varieti dello Strohel chiamando lo scheletro di Cortandone col nome di Baluenopteru Gastaldii, anzichè con quello di Plesiocetus Cortesii var. Cetotherium Gustalilii.

E naturale che io debba ginstificare il mio asserto dando una nuova descrizione del pezzo od almeno facendone risaltare i caratteri che mi inducono a questo ristabilimento di specie. Per questo ebbi aiuto potentissimo dalla pubblicazione del terzo fascicolo della Description des ossements fossiles des environs d'Anvers del Van Beneden arvenuta nel passato anno; per essa potei cercare nuovi caratteri stabili su cui appoggiare la mia diagnosi el assicurarmi che non davo troppa importanza a modificazioni individuali e che la Balcenopterc Gastaldii aveva piena ragione di esistere come specie effettiva. Veniamo dunque alle osservazioni da me fatte non più su disegni, ma bensi sull'originale stesso.

Teschio (fig. 3, 4, 5, 6). - Il teschio di Cortandone dovette misurare, allorchè era completo, circa m. 1,30 di lunghezza: infatti aggiungendo a m. 1,08, luughezza in linea retta della mandibola, la distanza dallo avanti allo indietro fra il bordo posteriore della cavitì articolare per la mandibola e l'angolo posteriore esterno dell'occipitale in m. 0,20, noi veniamo ad avere m. 1,28 per l'irtero cranio. La porzione conservata non misura invece che $m .0,95$, essendo mancante la porzione anteriore dei mascellari ed intermascellari e coll'arvertenza clie i punti collocati più allo indietro non trovansi, su questo cranio, sui condili di articolazione coll'atlante, ma bensi allo infuori di essi sugli angoli esterni dell'osso occipitale.

La maggior larghezza del cranio misurata dal punto più esterno del processo zigomatico di uno dei parietali a quello dell'altro raggiunge i $72 \mathrm{~cm}$.

Con queste piccole dimensioni e coi giovanili caratteri che vedremo or ora, questo teschio apparteneva già ad un individuo adulto; infatti le epifisi delle vertebre di tre delle regioni del corpo, la corvicale, la lombare e la caudale sono già completamente saldate e fuse coi rispettiri corpi di vertebra, e cosi pure il corpo dell'omero è già completamente unito alle proprie parti articolari sia superiore che inferiore ed il cubito è già saldato, senza traccia di primitiva separazione, alla prarte sua olecranica, mentre manca della epifisi inferiore che in molti individui anche adulti di varii generi di Cetacei non si individualizza dalla cartilagine arvolgente le ossa del carpo.

Uccipitule. - La forma e la posizione dell"occipitale sono oltremodo difficili al esprimersi con parole; cercherò però di spiegarmi il più chiaramente possibile. I due occipitali esterni, la di cui sutura divisoria dalloccipitale superiore è ancora parzialmente riconoscibile, sono collocati quasi verticalmente e guardanti allo indietro. Ciascuno di essi è, fra il condilo e l'angolo esterno proprii, poco profondamente e vastamente incarato. La curva, che presenta la concavità allo indietro, si continua fino all'angolo esterno dell'occipitale esterno, punto, come già arvertimmo, collocato il più allo indietro di tutto il cranio, sporgente di più di due centimetri più allo indietro del piano verticale passante pei punti posteriori estremi dei condili. li li l'osso occipitale estemo di ciascun lato si ripiega bruscamente in fuori ed in avanti e, dopo un percorso di appena quattro centrimetri, si nasconde sotto la sutura di articolazione col temporale. 
L'osso occipitale superiore per contro si puo nettamente dividere in due metà, l'una posteriore ed inferiore, l'altra anteriore e superiore, separate 'ta ma specie di cresta curva a concavità verso l'avanti. La metà posteriore è collocata quasi sullo stesso piano degli occipitali esterni, cioè quasi rerticale e guardante allo indietro. La sua parte mediana poi, che si abbassa molto nel foro occipitale e che si riduce ad avere una forma a mezza luna, è lievemente carenata longitudinalmente. Tale carena si estende poi allo insư, fino a perdersi nella metà anteriore clello stesso occipitale superiore che abbiamo precedentemente distinta e che invece di presentarsi collocata quasi verticalmente come l'inferiore, si presenta invece molto meno inclinata posteriormente e quasi vicina alla orizzontale. Essa è quasi pianeggiante ed un po' rigonfia ai lati, mentre la parte mediana è ampiamente scavata da un solco diretto dallo avanti allo indietro, nel quale viene posteriormente a perdersi la lieve carena che scorreva sulla prima metà dell'oșso stesso, e che si va lievemente dilatando allo avanti.

In complesso tutto il sistema occipitale, visto dal di dietro, si presenta come un triangolo molto inclinato allo indietro, arente una larga base e corrispondentemente una piccola altezza ed un vertice molto ottuso, i due angoli esterni ripiegati verso chi guarda il cranio dalla sua faccia posteriore e l'angolo superiore ripiegato in senso opposto; i due condili poi di articolazione per l'atlante sono pochissimo sporgenti ed il forame occjpitale si mostra con apertura gnardante allo inclietro ma molto allo insu, per cui vien naturale lo ammettere che il midollo spinale uscendo dal cranio ed immettendosi nel canale cervicale dovesse fare un angolo molto sentito li di cui sporgenza era rivolta in basso.

Da quanto dicemmo risulta chiaramente che la squama dell'occipitale non potevasi granchè estendere anteriormente sulle altre ossa del cranio e che quindi le ossa medie del-cranio stesso doverano mostrarsi alla luce per zone estese e molteplici come in seguito siamo per vedere.

Temporali. - Le ossa temporali sono oltremodo massiccie, e la loro apofisi giugale, facendo un angolo molto acuto colla parte posteriore dell'osso diretta allo infuori ed un poco allo indietro, si ripiega quasi direttamente allo avanti; essa andara molto probabilmente fino a contatto coll'angolo postero-esterno delle ossa frontali.

Nella faccia inferiore poi di ciascun temporale è scavata una profondissima fossa o condotto auditivo, diretto affatto trasversalmente dallo infuori allo indentro e sboccante contro un apparato auditivo curiosissimo per quanto ancor se ne veda.

Appurulo anditico (fig. 6 bis, 6 ter, 6 quater). - Infatti l'apparato auditivo destro è rimasto ancora in posto, e mentre le altre parti sono mascherate dalle ossa del cranio e dalla durissima roccia, ne sporge fuori la cassa timpanica cortissima e molto rigonfia ai lati, per modo che il diametro suo trastersale vien quasi ad eguagliare quello antero-posteriore. Essa non è appiattita inferiormente e, soltanto nella porzione inferiore della faccia interna, presenta una larga fascia un po' più rugosa. L'apertura della cassa, tuttora in parte mascherata dalla roccia che io non mi attento di estrarre, mostra essere piccolissima e sovratutto cortissima, e da essa discende lungo la faccia esterna della cassa un rigonfiamento a mo' di gobba, lungo (dallo avanti allo indietro del teschio) un centimetro e mezzo, sporgente quasi un centimetro e che si perde inferiormente nella convessità generale della cassa. 
La forma generale di quest'osso è assai ben visibile nella figura $3^{\mathrm{a}}$, tav. XXI, delle Untersuchungen ueber fossiten Cetaceen Europr's iel Brandt, rappresentante il teschio reduto dalla sua faccia inferiore; se ne vede assai bene la forma globosa, ma molti dettagli vanno perduti a cagione della forte riduzione del disegno. Essa è pure risibile nella mia fig. 4, dove il teschio è rappresentato alla scala di $1 / 4$.

Non conosco finora alcuna specio di Cetacei a fanoni viventi o fossili, la cui cassa timpanica si arricini pel la forma sua a quella della Buluenoptera Gastaldii, nessuna raggiungendo il grado di rigonfiamento e di brevità che distingue la cassa di questa specie. Soltanto nei Misticeti giovani la cassa timpanica si mostra più rigonfia e liscia (non mai però al punto di quella che ho sott'occhio), diventando poi man mano più compressa e carenata a misura che l'individuo avanza in etì. Per conseguenza la forma e disposizione dell'apparato uditivo della Balacnoptera Grastaldii serviranno con altri caratteri di cui più sotto si farì parola a dimostrarci come l'individuo tipo di questa specie, quantunque essendo già adulto o pressochè, conservava tuttaria alcuni distintivi che nella generalitì dei Misticeti appartengono agli individui giovani e cle nel nostro caso servono a caratterizzare la specie.

Alla stessa conclusione ci porta l'osservazione del rolume della cassa stessa in comparazione con quello di altri Misticeti molto più grandi. Infatti il cranio della Bulacnoptere (Plesiocetus) Cortesii scavato dal Gastaldi a Montafia, e di cui sarà parola in seguito, le di cui dimensioni erano quasi doppie di quello di cui ora si tratta, ci mostra una cassa auditiva non solo relativamente, ma ancora assolutamente piủ piccola di quella della Balacnoptera Gastaldii. Sappiamo che il rapporto di volume degli organi dei sensi con quello generale del cranio diminuisce generalmente coll'avanzare dell'età; quindi il conservare, contrariamente ai congeneri, un apparato uditivo cosi voluminoso ancora allo stato adulto, ci indica che la Balenottera di Cortanclone può venir considerata come specie tendente a conservare molti dei caratteri primitivi sotto ai quali apparve dapprima il tipo dei Misticeti, o che uscita da una branca già assai divaricata del medesimo tendeva a riarvicinarvisi.

Come effetto immediato poi di questo sviluppo dell' organo uditivo dobbiamo ancora ammettere che, a paritì di condizioni, la Balnenoptera Gastaldii dovesse arvertire i pericoli che la minacciavano a maggior distanza delle specie congeneri e quindi con maggior sollecitudine sottrarsi ad essi.

Paricluli. - Passando ora alla regione media del cranio, noi troviamo altri caratteri gioranili persistenti nel sistema parietale. Lo chiamo sistema poichè, quantunque come fu gii più volte ripetuto, appartenga ad un individuo adulto, pur tuttavia noi non abbiamo a fare con clue sole ossa risibili dallo esterno del cranio, ma bensi con tre per lo meno. Infatti, se noi partendo dall' angolo anteriore descritto dell' occipitale superiore ci avanziamo verso il rostro, troviamo immediatamente a contatto con l'angolo sopraoccipitale stesso la sutura occipito-parietale. Superata questa, noi percorriano un $4 \mathrm{~cm}$. d'osso e giungiamo ad una seconda sutura trasversale e dopo questa, fatti $3 \mathrm{~cm}$. di percorso, ad una terza che occorre ancora superare per trovarci sulla zona delle ossa frontali separata a sua volta, per mezzo di una quarta sutura, dalle ossa nasali, intermascellari e mascellari. 
La zona adunque che è interposta sulla linea mediana ctel cranio fra l'angolo supero-anteriore dell'occipitale e la origine posteriore delle ossa nasali misura non meno di nove centimetri di lungliezza, enorme questa se compariamo sotto tal punto di vista (ed avuto riguardo alle dimensioni di ciascun cranio) la Butacnoptera Gastuldii colle altre Balnenoptera virenti o fossili conosciute nelle quali tutte l'occipitale superiore tiene tanta parte nella region superiore del cranio. V'ha di piu, questa zona si dirile nattamente in tre zone ossee; di queste rediamo l'anteriore appartenre alle ossa frontali e la posteriore alle temporali, per modo che non ci resta che di consilerare la mediana come appartenente alle interparietali di cui ocсира la posizione.

Se, come ebbi cura di fare, noi segniamo con un colore qualunque il percorso delle diverse suture, sia nella regione mediana che estendendoci ai lati, in modo da ottenere visibile in un sol colpo d'occhio tutto il contorno di ciascun osso, noi scorgiamo come la zona appartenente ai parietali proprii si estenda da ciascun lato, sempre conservando l'aspetto di stretta benda, alla regione superiore e posteriore delle fosse temporali, seguendo il contorno della sutura parieto-occipitale sino presso al limite tra l'occipitale superiore ed il laterale di ciascun lato e come di li si diriga poi bruscamente allo ingiù; superato poi un vero stretto limitato allo infuori dall'osso temporale ed allo indentro dall'interparietale, si riespanda alqquanto per formare la metà infero-interna del lato posteriore della fossa temporale.

Le ossa interparietali invece, cortissime sulla linea mediana e sulla, qui stretta, superficie superiore del cranio, appena superato da ciascun lato il lieve spigolo di limite della superficie stessa, si estendlono alquanto allo avanti ed allo indietro ed acquistano cosi a detrimento dei parietali una certa quale espansione; ma dopo lieve percorso il lor margine anteriore si ripiega allo ingiu, poi allo indietro e le ossa interparietali vengono cosi ridotte ad una seconda zona, parallela alla prima, ma piủ irregolare e che si termina poi bruscamente da ciascuna parte contro allo stretto or ora descritto pel ciascuno dei veri parietali.

È noto, dai belli studii del Van Beneden sui Cetacei viventi e fossili, cone nei Cetacei giorani, dore è ancor riconoscibile la presenza ri una zona interparietale, allorchè si cerchi di stabilire le relazioni fra le diverse zone di ossa aftioranti sulla linea mediana del cranio, sia colla semplice osservazione dalla superficie, sia e meglio col taglio verticale lungo il piano di simmetria, si riconosca sempre una sorrapposizione di ossa l'uno all'altro per modo che la zona anteriore riene allo indietro, in parte o quasi completamente ricoperta dalla zona che la segue, e questa a sua volta dalla successiva fino alla occipitale la quale può tanto avanzarsi da mascherare quasi completamente sulla linea mediana le regioni parietale e frontale. Ora una tale osservazione è molto facile a farsi sul cranio che mettiamo per tipo della Balacnoptera Gustuldii: in esso noi vediamo chiaramente come, partendo dalla radice posteriore delle ossa nasali, la zona frontale mediana non abbia che due centimetri di lunghezza e renga quindi mascherata dalla sorrapposizione (precedentemente per far più presto e più chiaro la chiamai sutura assieme alle due altre di cui fu parola) della zona interparietale, come questa ancora non abbia dallo aranti allo indietro che tre centimetri di lunghezza, sendo mascherata allo indietro dalla sorrapposizione della zona parietale 
propria la quale finalmente a sua volta dopo quattro centimetri di lunghezza viene nascosta per sottoposizione all'occipitale superiore.

Nella indicata disposizione vediamo adunque, dopo quelli tratti dalla osservazione delle ossa occipitali e della cassa timpanica, un terzo carattere giovanile persistente nel cranio della Bataenoptera Gastatciii.

L'osso gingale naturalmente manca dalle due parti; non possiamo adunque farne cenno. Fa duopo invece che ricordiamo come il Brandt sia stato indotto in errore dai disegni del teschio di Cortandone che gli furono inviati, e che egli riprodusse nella tavola XXI del classico suo lavoro sopra citato. L'errore consiste nel seguente fatto: Il compianto Prof. Gastaldi, allorchè attese a spogliare dalla roccia il cranio di Cortandone, vedendo come il processo zigomatico destro fosse rotto aranti la sua terminazione anteriore e come fra il pezzo ancor rimanente e la porzione esterna del margine posteriore del frontale si fosse formata una roccia sabbiosa solidissima, con molta accortezza ed allo scopo di conservare a queste porzioni più sottili e sporgenti del cranio una certa qual consistenza, non tolse affatto tale frammento di roccia, ma bensi lo rìlusse e lo tagliò facendone una specie di pilastro orizzontale che collega il temporale al frontale. Il disegnatore Rapetti, nel far la figura del cranio, non pensò forse ad ommettere questo pilastro di pura precauzione o, disegnandolo, a farlo risaltare come pezzo appartenente solo all'armatura e che quindi non doveva entrare in calcolo nello esame del fossile. Ne venne che il Brandt considerò un tal pilastro come appartenente al fossile stesso, la forma e la posizione sua nel puro disegno rendendo molto naturale l'illusione, lo ritenne come l'estremità anteriore del processo zigomatico del temporale e come tale lo fece riprodurre nel suo disegno e ne parlò nel suo esame, inducendo cosi involontariamente in errore quanti ne parlarono in seguito sulla sola base della sua figura senza aver visto l'originale. Fra questi possiamo citare lo Strobel che riprodusse il disegno e tenne in linea di conto anche questa erronea direzione del processo giugale. Quest'ultimo invece era bensi rivolto allo avanti, ma prolungato sarebbe venuto a toccare il margine posteriore del frontale alquanto più allo infuori di quanto sia stato finora ammesso.

Riparato cosi a tale errore, torniamo allo esame delle altre ossa del cranio.

Fromlali. - Le ossa frontali, cortissime sul piano mediano del cranio, si estendono sui fianclii molto più allo indietro formando gran parte dei lati interni delle fosse temporali a scapito delle ossa parietali (ed interparietali) che respingono molto allo indietro. La sutura posteriore della parte verticale dei frontali stessi coincide così col fondo delle fosse temporali. Dal fondo della fossa temporale poi si stacca la porzione orizzontale, od espansa, od orbitale dei frontali stessi il cui margine posteriore arrotondato e quasi rettilineo è diretto di dentro in fuori e lievemente inclinato allo avanti, mentre il margine anteriore è ampiamente incurvato colla convessità rivolta allo avanti.

Il frontale destro è quello più ben conservato: una trasversale dal fonclo della sua porzione verticale al presente margine orbitale di rottura (che probabilmente è allo indentro dal margine orbitale naturale e primitivo di circa due centimetri) misura 22 centimetri di lunghezza. Se esaminiamo poi la porzione espansa dalla sua faccia inferiore vediamo come essa sia ricoperta nella sua metà anteriore dal mascellare, 
mentre la metì posteriore è affetta dapprima al passaggio del nervo ottico poi alla carità orbitale. Tutto ciò per far notare come la clirezione del nervo ottico fosse quasi normale alla lunghezza del cranio e come esso corra quasi in un cartoccio osseo che, per un buon terzo interno della parte espansa del frontale, è quasi completamente chiuso non lasciando in basso che una strettissima fessuria; questa si va poi gradatamente ampliando nel resto della parte espansit ed in egual misura si svolge l'indicato cartoccio osseo per dar lnogo alla arcata orbitale che ancora alla estremità è profondamente marcata nella faccia inferiore dellosso.

dllo stesso modo delle ossa jugali mancano entrambe le ossa lacrimali.

Iirsuli. - Le ossa nasali non sono perfettissimamente conservate sopratutto anteriormente, tuttavia se ne riconosce assai bene la forma e la posizione. Esse sono allungatissime e molto strette; alla loro origine ciascuno è arrotondato per modo che un piccolo angolo anteriore della zona frontale si frammette alle due ossa; esse si estendono poi allo avanti acquistanclo pochissimo in larghezza per modo che il loro bordo esterno si mantiene quasi parallelo alla linea mediana ed il margine anteriore dei lue fa, a quanto pare, un angolo lievemente aperto allo avanti.

Intermuscelluri. - Le ossa nasali non toccano allo esterno i mascellari che per il quinto posteriore della loro lunghezza; nei quattro quinti anteriori sono invece come incorniciati dentro a sottilissime apofisi posteriori degli intermascellari le quali si interpongono fra esse el i mascellari, acquistano, man mano che ci aranziamo, in larghezza ed altezza, circondano poi, dopo il margine anteriore delle ossa nasali, l'ampia fossa nasale mantenendosi rerticali per rispetto ad essa e facendo sui fianchi ed a pochi centimetri allo avanti del limite delle ossa nasali, ciascuno, un piccolo tubercolo allungato: oltrepassato questo si innalzano vieppiù, si ingrossano ed accennano a convergere, come convergono difatti molto più allo avanti, chiudendo anteriormente la fossa nasale ed assumendo l'aspetto di due grossi semicilindri l'uno all'altro addossato e compenetrato e rinchiudendo l'astuccio in cui era contenuta la lamina cartilaginosa dell'etmoide e mascherando tutta la porzione anteriore del vomere.

T'utto il terzo anteriore del rostro è poi mancante, per modo che non sappiamo in quale reciproca disposizione terminassero e le ossa ora descritte e le mascellari.

Iluscellari. - Di queste ultime possiamo dire soltanto, come, per mezzo di assai larga apofisi posteriore, si frappongano fra i frontali e gli intermascellari nella regione latero-nasale, rimontino piu in su degli intermascellari fino a toccare la zona mediofrontale quasi sulla stessa trasversale del margine posteriore delle ossa nasali; come la loro parte latero-posteriore espansa circondi, sulla faccia superiore, il borlo convesso anteriore della parte orbitale dei frontali, mentre sulla faccia inferiore ne ricoprano metà e come di li, mantenendo una posizione costantemente e lievemente piana e declive allo infuori per la superficie superiore, pianeggiante ed inclinata allo insù per la faccia inferiore, entrino cogli intermascellari nella composizione del rostro, come si è detto anteriormente.

Fuccill inforiore del crmmio. - Sulla faccia inferiore del cranio noi scopriamo poi, oltre alle ossa già nominate: l'occipitale basilare conservatissimo (del quale fu già in parte discorso a proposito dei condili e del forame occipitali), porzione dello sfenoide, il palatino destro schiacciato contro allo sfenoide ed al presfenoide, e, fram- 
mezzo ai due mascellari, porzione posteriore del romere. Tali ossa hanno ad un di presso la forma e la posizione che siamo arrezzi a riconoscere nel cranio delle $\mathrm{Ba}$ lenottere in generale e non meritano di essere specialmente considerate, limitandoci a far osservare come il vomere abbia dovuto nella parte anteriore (nei due terzi) del rostro essere completamente mascherato sulla sua faccia inferiore dall'espansione dei due mascellari che renivano a toccarsi sulla linea mediana.

II andibole (fig. $7,8,9,10,11,12$ ). - Una parte del cranio che, dopo gli studii del Van Beneden, ha invece acquistata una importanza grandissima per la determinazione dei generi e delle specie di Misticeti viventi e fossili si è la mandibola. E lo scheletro di Cortandone ci mostra queste due ossa perfettissimamente conserrate e tali da poterci formire una buona dose di caratteri utilissimi nelle nostre ricerche.

Le mandibole di questo individuo furono già figurate dal Brandt nelle sue $L$ ntersuchungen, tav. XXI, fig. 28 viste di finnco e fig. 29 viste di fronte; però la sua stessa fig. 29 è dal Brandt, come osserrarono il Van Beneden e lo Strobel. male interpretata, inquautochè nella spiegazioue delle tavole egli la dà come quella del primo paio di coste. Oltracciò l'esecuzione stessa delle figure lascia molto a desiderare e col solo aiuto di quelle noi non potremmo mai farci un idea esatta della forma e dei caratteri dei pezzi riprodotti. Ho quindi, partendo da altro punto di rista e con altri mezzi ed in scala meno ridotta, fatto riprodurre e l'intiera mandibola e le parti piu salienti di essa, come si vele nelle figure $7-12$, e spero che queste potrauno maggiormente servire alla illustrazione del nostro bel fossile piemontese.

La lunghezza della intiera mandibola destra, misurata da un capo all'altro in linea retta, è di 110 centimetri, misurata invece con un nastro lungo il bordo interno essa rișulta di $\mathrm{cm}$. 115. La corda tirata dal margine anteriore del foro d"origine del canale dentale alla estremitì anteriore della mandibola misura $95 \mathrm{~cm}$. di lunghezza, la massima saetta cosmpresa fra questa corda e la faccia interna della mandibola, misura nientemeno di $134 \mathrm{~mm}$. di lunghezza: abbiamo dunque a fare con una mandibola potentemente incurvata e tale da ricorlarci (per questo solo carattere) piuttosto quella dei veri Balenidi.

Oltre a tale curvatura, che comincia a farsi sentire allo innanzi del foro d'origine del canale dentale, non si osserva nella mandibola la distorsione per cui anteriormente la faccia interna abbia poi a diventare superiore, come nelle Negattere; una lieve tendenza a ciò la incontriamo soltanto nei dieci centimetri estremi anteriori dell'osso, ma solo risibile a chi ricerchi appositamente un tale carattere. La faccia interna dell'osso, piana nella regione posteriore, sotto al condilo e sotto alla origine del canale dentale, è lievemente concava in senso longitudinale nel tratto compreso fra l'origine del canale stesso ed una diecina di centimetri allo avanti dalla apofisi coronoide; più allo avanti ridirenta piana e poi subito lievemente piano-convessa, conservandosi tale fin quasi alla estremitì anteriore dove diventa un po' più convessa ed irregolare.

La faccia esterna dell'osso, piano-convessa nella regione posteriore, prima dell'origine della curvatura, diventa, coll'accusarsi di questa, fortemente convessa specialmente nella metà inferiore, si conserva tale pei due terzi posteriori della porzione curva; allo aranti scema alquanto di convessità, ed affatto auteriormente riesce poi a diventare quasi piana e rerticale. 
La faccia interna e la esterna sono nettamente l'una dall'altra distinte per mezzo dei bordi superiore ed inferiore, questo alquanto arrotondato nella regione posteriore, diventa poi sentitissimo e come tagliente appena superato il terzo posteriore della lunghezza della mandibola, e tale si conserva con lievi modificazioni fino alla estremità anteriore: quello parte taglientissimo dalla faccia anteriore del condilo, supera una lieve apofisi collocata al disopra del margine anteriore dellimbocco del canale dentale, fa una lieve curva allo ingiu che rimonta per superare la piccola apofisi coronoide, di lì continua, quando più, quando meno sentito, fino alla estremità anteriore presso alla quale si fa alquanto piu ottuso ed esterno per lasciare spazio internamente alla terminazione del canale dentale.

E prima ancora li passare ai caratteri delle parti, conviene che facciamo menzione di un ultimo carattere generale dell'osso, non meno importante dei precedentemente esposti, cioè la più o meno grande eguaglianza di altezza nelle diverse regioni di ciascun ramo di mandibola.

Chi osservi dalla faccia interna uno dei rami mandibolari collocato orizzontalmente ed all'altezza dei propri occhi dinanzi a sè, non tarda a rimarcare quanto sieno peculiari a questa specie le condizioni di altezza nelle diverse regioni. Infatti trascurando la parte non ricurva e partendo dall'apofisi coronoide, noi vediamo come questa appaia pochissimo sviluppata e confondentesi anteriormente nel bordo superiore generale il quale, mentre l'inferiore si mantiene sempre sullo stesso piano, va lentissimamente ed uniformemente rliscendendo fino oltrepassata la metà lunghezza della porzione ricurva.

Superato questo minimo di altezza del ramo mandibolare, il bordo superiore tende, ancora più lentamente di prima ma altrettanto uniformemente, a risalire, finchè la porzione anteriore del ramo mandibolare finisce per essere visibilmente pii alta della porzione mediana.

Se poi passiamo ai caratteri speciali, essi ci si affollano dinanzi così numerosi ed importanti da non sapere da quale cominciare per la descrizione. La porzione posteriore non ancora ricurva di ciascun ramo è quella che prima attrae la nostra attenzione. Noi segnaliamo dapprima, superiormente, un relativamente piccolissimo condilo di articolazione col cranio, collocato trasversalmente al ramo, ed avente circa $90 \mathrm{~mm}$. di larghezza; esso è munito posteriormente ed un poco superiormente della sua faccia articolare decisamente convessa. Tale condilo somiglia alquanto a quello della Balcenoptera musculoüdes di Anversa, è però, astrazion fatta dalle dimensioni assolute, più elevato e men largo ed in generale molto più distinto dal corpo dell'osso mentre la superficie articolare tende più ad avanzarsi nella Balaenoptera Gastaldi che nella $B$. musculoüdes.

Al di sotto del condilo articolare, la parte posteriore di ciascun ramo mandibolare si termina allo indietro in una specie di tallone assai pronunciato e sporgente di alcuni millimetri di più che il sorrastante condilo; questo tallone appiattito allo indentro, convesso allo infuori e con una faccia posteriore che si restringe man mano verso il basso, si arrotonda poi per raggiungere il bordo inferiore del ramo mandibolare, ma prima di confondervisi forma ancora un piccolo tubercolo inferiore, allungato, sporgente al disotto del bordo generale inferiore di almeno un centimetro e collocato verticalmente in corrispondenza della metà anteriore del condilo. 
Il tallone posteriore è poi separato dal condilo, sulla faccia interna, da una lieve depressione longitudinale, dore passavano i nervi e vasi immettentisi nel canale dentale; questa è perỏ molto meno prouunziata clıe non nelle Balenottere di Anversa e sovratutto non è, come in quelle, completata dal rilievo osseo longitudinule corrente lungo il margine superiore interno del tallone fino ad imboccare quasi l'apertura del suddetto canale. Tale depressione non è, nella Bulaenoptera Gustalulii, visibile che a chi la ricerchi espressamente: essa passerebbe senza di ciò aftatto inosservata e confusa nella faccia piana interna di questa parte della mandibola.

L'intaglio sigmoide è, nella specie piemontese, tutt'altro che regolare, inquantochè a metà lunghezza di esso, e precisamente sopra al margine anteriore dell'imbocco del canale dentale, noi vediamo il margine dell'intaglio sigmoide interrotto da una piccola protuberanza, una specie di apofisi precoronoide o coronoide accessoria, dopo la quale il bordo superiore si abbassa, fa una nuova curva allo ingiù e riascentle poi per raggiungere la vera apofisi coronoide pochissimo sriluppata, appiattita e che, vista dalla faccia interna, parrebbe collocata verticalmente, mentre, vista dalla faccia esterna o dalla superiore, si mostra invece leggermente ricurva allo infuori.

Un'altra modificazione degna di nota osserviamo ancora in questa regione della mandibola, ed è che sulla faccia interna dell'osso, dal margine anteriore dello imbocco del canale dentale, si stacca, ad un paio cli centimetri al di sotto della sommitì della descritta apofisi precoronoide, un rilievo osseo dapprima quasi insensibile, poi man mano sempre più forte, il quale scorre verso l'avanti inclinando alquanto allo insù e dopo aver raggiunto un massimo di sporgenza in corrispondenza della seconda metì dello intaglio sigmoide va poi, sempre continuando la sua direzione rettilinea, rapidamente scemando di sporgenza, sino a perdersi completamente sotto la piccola apofisi coronoide. Esso pare un riliero di rinforzo alla lamina ossea nella quale è compresa la metà anteriore dello intaglio sigmoide, la quale a vero dire sarebbe qui assai sottile.

Finalmente osserviamo, sempre nella regione posteriore della mandibola, sulla faccia interna e nella metì sua superiore, al davanti del condilo, la vasta apertura d'ingresso nel canale dentale; essa è obliqua d'alto in basso e di dentro in fuori, è collocata relativamente molto allo indietro, ed acquista ben presto, anche dal lato interno, una parete propria che la masconde allorchè il canale dentale ha presa una direzione determinatamente orizzontale. Dal momento della sua scomparsa posteriormente, il canale dentale non mostra più allo esterno traccia alcuna di sè che dopo aver superato il terzo posteriore del ramo mandibolare; di qui egli torna a mostrarsi quale leggiero solco sulla faccia interna dell'osso, ad un mezzo centimetro circa dal bordo superiore per tutta la lunghezza anteriore del ramo e conserrandosi dapprima sempre uguale e leggiero per lungo tratto, accusandosi poi più e più quando ci avviciniamo alla estremità e finalmente, giunti ad un sei centimetri da questa, si apre e si approfonda improvrisamente, sostituendosi al bordo superiore che respinge allo infuori, e sulla faccia anteriore di ciascun ramo discende poi verticalmente per un terzo dell'altezza e quindi si termina bruscamente ripiegandosi allo infuori.

Più in basso, sotto la metà altezza della faccia interna di ciascun ramo, veclesi partire dalla estremità anteriore dell'osso e dirigersi orizzontalmente allo indietro un 
altro solco, il quale, dopo breve percorso, svanisce e si perde nella superficie generale interna. Tale solco la una direzione e disposizione affatto simili a quelle che si osservano nella stessa posizione sulla Balacnoptera muscoloüdes di Anversa e non manca di averne la identica significazione.

Noterò finalmente sulla superficie esterna convessa di ciascun ramo e nella meta loro anteriore dei forami mentonieri tanto più allungati quanto essi son più collocati allo avanti e dei quali ne contai cinque in un ramo e sei nell'altro.

Sul bordo inferiore della mandibola non è in alcun punto riconoscibile traccia veruna di un solco longitudinale.

Raccogliendo i fatti che risultano da questa descrizione forse un po' minuta delle mantibole ne risulta che la forma del condilo è strettamente secondo il tipo Balacnoptera, che la forma del tallone ravricinerebbe un po' più il nostro individuo al tipo Burtinopsis del Van Beneden, che l'apertura e posizione dello imbocco del canale dentale e del solco per il passaggio dei tronchi venosi e sanguigni tra il condilo ed il tallone rammentano di più analoghe disposizioni nelle Balenottere viventi che non nelle fossili, che il poco sviluppo dell'apofisi coronoide rammenta più il tipo Balacna, che la divisione dell'intaglio sigmoide in due porzioni con sviluppo di un'apofisi coronoidea accessoria e li presenza di un rilievo di rinforzo alla metà anteriore dello intaglio stesso sono caratteri proprii alla nostra specie, che caratteri proprii sono ancora il decrescimento ed il successivo accrescimento dell'altezza di ciascun ramo mandibolare dallo indietro in aranti, che la curvatura dei rami sia più esagerata che non in generale per il tipo Balaenoptera senza raggiungere ancora il grado di tal carattere nel tipo Balacna, che infine il percorso del canale dentale, la sua terminazione in avanti, la forma, posizione, numero dei forami mentonieri e la forma della porzione estrema di ciascun ramo nonchè la presenza e percorso del secondo solco interno su di esso siano decisamente caratteri di Balacnoptera.

Noi saremmo quindi autorizzati a considerare la specie Balacnoptera Gustaldii, la quale raduna in sè i caratteri di tanti generi oggidi perfettamente distinti, siccome uno dei tipi chiamati, ed a ragione, complessivi.

Passiamo ora rapidamente in rivista le altre parti che ci vennero conservate dello scheletro di Cortandone.

Allante (fig. 13, 14, 15). - L'atlante, alquanto mancante alla sommitå dell'arco neurale ed alla estremità delle apofisi trasverse, è invece molto ben conservato nella sua parte inferiore. Il massimo suo spessore, misurato sulla faccia inferiore ad un paio di centimetri in fuori della linea mediana, giunge a quarantacinque millimetri; le due faccio articolari anteriori sono separate in basso l'una dall'altra da uno spazio di almen $18 \mathrm{~mm}$. di larghezza, molto vasto cioè in proporzione delle dimensioni generali dell'osso; le faccie stesse sono molto concave e molto estese in confronto delle dimensioni dei condili occipitali coi quali articolavano.

Le faccie articolari posteriori sono presso a poco pianeggianti, ciascuna di esse è lievissimamente inclinata allo infuori ed è in basso separata dall'altra da uno spazio intermedio ancora più grande che per le faccie anteriori, raggiungendo fin $47 \mathrm{~mm}$. 11 foro vertebrale è formato come la cifra 8 caporolta, inquantochè la sua metà inferiore è molto piủ ristretta della superiore, il suo bordo inferiore però è ancora 
arrotondato, non mai ristretto ad angolo; la metì superiore è larghissima e come schiacciata d'alto in basso.

I fori che attraversano i pedicoli dell'arco neurale sono proporzionalmente larghissimi, l'apofisi spinosa dovette essere ridotta, da ciò che si vede, ad un lieve tubercolo, e le apofisi trasverse sono abbastanza forti, sviluppate el espanse allo insù.

L'atlante della Balcenoptera Gastaldii ci ricorderebbe, per la delicatezza sua e pel contorno esterno, più che ogni altro, quello della Balacnoptera rostratella V. Ben. di Anversa; ne differisce però a prima vista per la sua maggiore altezza e minor larghezza, per la maggior distanza fra le due grandi faccie articolari anteriori e sovratutto per la differenza di forma della parte inferiore del foro vertebrale, ristretta ad angolo nella $B$. rostratella, arrotondata nella $B$. Gastciliii. Sotto questo rapporto la B. Gastalctii si accosterebbe dippiù alla B. (Plesiocetus) Cortesii, di cui parleremo in seguito, quantunque in questa seconda il forame finisca per essere relativamente più basso e schiacciato che nella prima, oltre a tante altre differenze che vedremo poi parlandone di proposito.

Asse (fig. 16, 17, 18). - Il corpo della vertebra asse, che è ammirabilmente conservata, ha uno spessore press'a poco uguale a quello dell'atlante, la sua apofisi odontoile è pochissimo sviluppata ed è coperta nella metà inferiore da una benda di faccia articolare che unisce il basso delle due faccie articolari che le stanno accosto; queste sono molto debolmente concave ed inclinate allo indentro, ciò corrispondentemente alle posteriori dello atlante che erano invece inclinate allo infuori.

L'arco neurale è costituito da una lunga volta, a pareti relativamente sottili e non ingrossate che alla sommitì formano il bulbo dell' apofisi spinosa che non doveva elevarsi gran che. Le faccie articolari per larco della terza vertebra sono ben visibili, sviluppate ed ovali. Il foro vertebrale è ampissimo, fatto come la lettera $O$, un po' dilatata a meta altezza, il fondo del canale neurale è fortemento declive allo avanti. Le apofisi trasverse superiori sono entrambe rotte alla estremita, esse erano costituite da un'ampia lamina molto espansa allo infuori, che deve discendere fino ad incontrare le trasverse inferiori colle quali veniva formato un ampissimo anello da ciascuna parte del corpo della vertebra. Le apofisi trasverse inferiori, forti e triangolari alla origine, si espanderano grandemente allo infuri assumendo esse pure ad una certa distanza l'aspetto di forte lamina collocata verticalmente e trasversalmente alla linea mediana e circoscrivento in basso ed in fuori gli anelli laterali di cui fu parola.

Chiudiamo la descrizione di questa vertebra con dire che la faccia sua articolare posteriore è decisamente concava e che la epifisi corrispondente (già completamente saldata al corpo (lella vertebra) sborda tutto allo intorno del corpo, estendendo così viemaggiormente la superficie della faccia stessa.

Possiamo ripetere per la seconda vertebra cervicale della Balacnoptera Gastaldii, comparata con quella della $B$. vostratella, quello che già dicemmo a proposito dello atlante, che cioè nella $B$. Gastaldii essa è piủ elevata e ristretta e che tale carattere, assai sensibile allorchè si faccia solo attenzione al profilo generale dell'osso, lo diviene maggiormente allorchè si comparino una per una le parti, specialmente il foro vertebrale e la gran faccia articolare posteriore. 


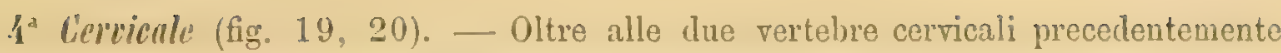
descritte, noi ne abbiamo ancora una terza, che dai caratteri suoi non pare essere stata quella immediatamente successiva all'asse, ma più tosto la quarta o la quinta. $\grave{E}$, relativamente, assai piccola el il corpo ne è molto sottile non misurando dallo aranti allo inlietro più di $23 \mathrm{~mm}$. nei punti cli maggior spessore. La faccia anteriore del corpo è decisamente piana con lieve impressione concentrica ad un centimetro dalla periferia, la posteriore invece è lievemente concava e con bordo superiore ed inferiore più sentiti dei laterali.

La figura delle due faccie è quadrangolare, con diametro trasversale di poco piu ampio del verticale e con angoli arrotondati. Le epifisi sono gia completrmente saldate al corpo e non si vede altra traccia della loro primitiva esistenza che sui bordi delle faccie articolari sporgenti molto piu allo infuori del corpo della vertebra. apparendo questo come scavato tutto allo intorno. Sulla faccia inferiore poi del corpo stesso vedesi lungo la linei mediana una lieve carena arrotondata.

Il canale neurale era ampissimo e pare fosse molto schiacciato: dico pare, poichè a noi manca la parte mediana dell'arco neurale. I due pedicoli che ci rimangono dimostrano come l'arco consistesse in una forte lamina schiacciata dallo avanti allo indietro e fortemente ripiegata allo indietro nella parte sua mediana, per modo che le faccette articolari per l'arco della vertebra precedente sono molto allungate, poco inclinate allo avanti e guardanti piuttosto allo insù, quelle per l'arco della vertebra consecutiva sono alle prime quasi parallele.

I processi trasversi superiori nascono dai pedicoli dell'arco neurale, sono essi pure appiattiti d'aranti in dietro e diretti affatto all'infuori; entrambi sono rotti all'estremità, non si può dire per conseguenza di quanto essi fossero sviluppati.

I processi trasversi inferiori nascevano invece molto in basso, quasi dagli angoli latero-inferiori del corpo el erano diretti più allo ingiu che in fuori; non pare abbiano avuto un grande sviluppo, probabilmente invece averano la forma di grossi tubercoli.

Anche questa vertebra, confrontata con quella delle specie di Balenottere fossili a me note, mostra le sue grandi faccie articolari molto più elevate in proporzione della larghezza; quindi più decisamente quadrate che in tutte le suddette specie.

lertebi't lombare (fig. 21, 22). - Noi non abbiamo alcuna delle vertebre dorsali dello individno di Cortandone; ne abbiamo all'incontro una sola spettante alla regione lombare media; in essa, che è ridotta al solo corpo, le epifisi sono completamente fuse alla diafisi e fanno un leggiero ribordo tutto attorno di essa, le due faccie articolari sono quasi perfettamente piane. Il corpo della vertebra è molto allungato $(114 \mathrm{~mm}$.$) el esteso trisversalmente (115 \mathrm{~mm}$. $)$ in proporzione di quanto è alto $(83 \mathrm{~mm}$.$) : le sue faccie latero-superiori e latero-inferiori sono sensibilmente incarate,$ e sulla linea mediana, inferiormente, esso è sentitamente carenato.

Il canale neurale è ancora assai vasto e le apofisi trasverse, assai forti ed appiattite, avevano la loro origine al di sopra della meta altezza del corpo. È notevole di questa vertebra la gran lunghezza ed il gran diametro trasversale.

Vertebre cunduli (fig. 23, 24). — Fra le quattro vertebre caudali di questo scheletro ancora conservate, una appartiene alla regione mediana inquantochè le 
apofisi trasverse, ancora forti abbastanza, cominciano ad essere perforate dai vasi arteriosi ; il suo corpo misura $100 \mathrm{~mm}$. di altezza per $120 \mathrm{~mm}$. di larghezza (misurate sulla faccia articolare posteriore) e per $116 \mathrm{~mm}$. di lunghezza. Tanto la faccia articolare anteriore che la posteriore sono al centro lievemente concave : le faccie superoed infero-laterali profondamente incavate, le epifisi sporgenti allo intorno ma completamente saldate al corpo.

Sulla faccia inferiore $\mathrm{i}$ tubercoli di articolazione per l'osso a $V$ anteriore sono pochissimo sviluppati a quasi nulli; sviluppati, sporgentissimi e compressi dai lati sono invece i tubercoli per l'osso a $V$ posteriore; essi fanno un risalto di più di due centimetri di altezza.

I pedicoli dell'arco neurale molto ravvicinati, compressi dai lati, circoscrivono colla parte mediana dell'arco un foro rachidiano molto più alto che largo e quasi perfettamente ovale. I due processi articolari per l'arco neurale della vertebra antecedente, quantunque ancora ben sviluppati, non portano più traccia di faccetta articolare: l'apofisi spinosa fortemente ripiegata allo indietro e compressa dai lati non dovera più estendersi molto allo insù.

Anche nelle vertebre di questa regione si fa adunque sentire, contrariamente a ciò che succede per molte altre Balenottere fossili, la prevalenza del diametro trasversale del corpo su quello longitudinale e sovrattutto di entrambi questi due sul verticale.

Le altre tre vertebre caudali che possediamo sono l'una all'altra consecutive, appartengono alla estremità della pinua e probabilmente la terza di esse non era più, nel viro, seguita che da una sola vertebra piccolissima e deforme. In essa le apofisi trasverse ed i processi neurali sono rilotti a tozzi tubercoli; il solo canale emale vi è ancora molto bene determinato ed affondato; sono, come di regola, attraversate sui fianchi verticalmente d'alto in basso dai fori in cui passarano i vasi arteriosi; la loro forma rammenta quella. di tronchi di cono aperti allo avanti colle faccie anteriore e posteriore quasi piane; l'ultima caudale conservata non ha anzi quasi più. forma determinabile.

Costo (fig. 25, 26). - Oltre alle parti accennate ci restano dello scheletro di Cortandone due coste sinistre, l'una di esse è probabilmente la seconda, l'altra la quarta o la quinta. La seconda costa misura, secondo la curva del bordo superiore ed esterno, centimetri 80 ; essa è molto appiattita e contorta sulla metĩ prossimale della faccia anteriore, mentre l'altra metà è decisamente convessa; la superficie posteriore è invece tutta pianeggiante e leggermente convessa, il bordo inferiore è molto pronunziato e quasi tagliente, il superiore invece arrotondato, la larghezza media dell'osso è di circa $55 \mathrm{~mm}$. Verso l'estremità distale questa costa si restringe alquanto, poscia si riespande per un tratto di un decimetro di lunghezza e torna a restringersi alquanto nei due centimetri estremi.

La costa presenta nella porzione prossimale una faccia articolare lunga e stretta per l'apofisi trastersa el anteriormente a questa si ristringe in un collo che doreva portare il capitolo per l'articolazione col corpo della vertebra, ma che sgraziatamente è rotto ad un centimetro al davanti dal margine anteriore del tubercolo. Non possiamo per conseguenza conchiudere se riescisse o no a raggiungere e toccare il corpo stesso. 
L'altra costa, che abbiamo detto essere la quarta o quinta di sinistra, è quasi completa: lungo il borlo esterno essa misura $78 \mathrm{~cm}$. di lunghezza a cui se ne potranno aggiungere un 4 o 5 per l'estremitì distale che manca. La sua larghezza media è di $38 \mathrm{~mm}$; essa ha la faccia anteriore rigonfia o lievemente pianeggiante nel terzo prossimale, molto convessa negli altri due, la faccia posteriore lievemente convessa, i due bordi molto sentiti, specialmente l'inferiore clıe è quasi tagliente. Essa porta sulla sua.porzione prossimale la faccetta di articolazione tubercolare per il collegamento alla corrispondente apofisi trasversa el, al davanti del tubercolo, la costa, come già dicemmo per l'altra, si restringe in un collo lungo, dal margine anteriore del tubercolo, un $6 \mathrm{~cm}$.; questo va vie piu assottigliandosi e si termina in un piccolo capitolo arrotondito, che non porta alcuna impronta articolare; è dunque probabile che non arrivasse al corpo della vertebra che mediante un prolungamento cartilagineo la di cui supposizione è confermata dalla natura porosa del capitolo stesso.

Noi sappiamo come, per le Balenottere in generale, le prime sei coste da ciascuna parte abbiano, oltre al tubercolo, una parte prossimale che rappresenta il collo ed il capitolo, come raramente questa riesca a toccare il corpo dalla vertebra e come fra queste sei coste quella che piu sovente riesce a toccare il corpo stesso sia appunto la quarta; gli è quindi naturale che noi, dallo esame di tale parte nella presente costa e dai suoi caratteri di minore allargamento e di maggiore lunghezza, veniamo a conchiudere aver essa dovuto tenere il quarto o quinto posto nella serie delle vertebre di sinistra.

Joile (fig. 27). - Assieme alle altre ossa, allorchè venne scavato lo scheletro di Cortandone, venne rinvenuto un piccolo osso lungo rilotto a frammento che non fu mai determinato perchè non veune mai considerato come oggetto di valore; venne però sempre tenuto assieme alle altre ossa della stessa provenienza ed individuo. I'rovatolo, e dopo averlo vanamente comparato colle piu clisparate parti di varii animali, venni a riconoscere che esso non era altro che uno dei corni posteriori dell'osso joide di un Misticeto e che aveva per conseguenza dovuto appartenere alla stessa Balacnoptera Gustaldii con cui era stato rinvenuto, tanto piu che colla medesima si accordava anche per il piccolo suo volume. Abbiamo così dinanzi la metà destra del pezzo posteriore del joide relativamente assai bon conservata: ne rileviamo che l'intiero osso joide era appiattito, el assai allungato allo avanti sulla linea mediana, e che le due branche si andavano arrotondando verso l'estremità per terminare in una tuberosita irregolarmente incavata allo esterno ed allo indietro per ricevere la cartilagine che le completava allo esterno. L'osso che abbiamo dinanzi, quantunque riconoscibile come metà di un osso joile, non è però affatto isomorfo con quello di alcuna specie di Misticeto vivente o fossile, come si potrà rilevare dalla figura 31; esso mostra soltanto una lontana analogia di forma e di posizione coll'osso joide della Balaenoptera Sibbaldii.

Delle ossa delle estremità mancano a noi entrambe le scapole, che ci sarebbero state cosi utili; e non abbiamo in tutto che un omero, un cubito ed ossa della mano.

Omero (fig. 28, 29). - L'omero destro che noi possediamo è straordinariamente piccolo, essendo completamente compreso in una lunghezza di $167 \mathrm{~mm}$. erl, in cor- 
rispondenza del maggiore sviluppo del condilo, in una larghezza di $96 \mathrm{~mm}$., mentre lo spessore massimo del corpo dell'osso al di sotto del condilo non tocca che $46 \mathrm{~mm}$.

Distinguono questosso: il condilo e la faccia articolare di esso relativamente assai sviluppati e molto inclinati sul bordo superiore e sulla faccia esterna del corpo e separati tutto allo intorno da un embrione di collo, la tuberosità inferiore molto sviluppata e sporgente allo ingiù, il corpo dell'osso cortissimo, molto appiattito e mostrantesi come diviso longitudinalmente in due parti disuguali da una profonda impressione longitudinale collocata sulla faccia esterna tra la linea mediana ed il bordo inferiore ed alla quale corrisponde sulla faccia interna un'altra impressione più obliqua e piủ avricinata alla linea mediana, l'estremità distale poco espansa mostrante le due faccie articolari per le ossa dell'avambraccio clie fanno fra loro un angolo molto ottuso mentre la faccia articolare accessoria pel cubito è parallela all'asse dell'osso. Niun Balenotteride virente o fossile a me noto presenta un omero con caratteri uguali a quello della Balacnoptera Gastaldii e sorratutto il cui condilo sia così potentemente inclinato relativamente al corpo dell'osso. Hinori analogie ancora troviamo poi coll'omero delle Megattere o delle Balene.

Cubito (fig. 30, 31). - 11 cubito sinistro che abbiamo dinanzi è compreso in una lunghezza di $271 \mathrm{~mm}$. (compresa l'apofisi ascendente olecranica); la sua lunghezza sta per conseguenza a quella dell'omero nel rapporto di 1,6-1. Esso si presenta coll'aspetto di un osso allungato, a sezione irregolarmente elissoidea, pochissimo compresso; il lato che guarda il radio è un po' appiattito superiormente e munito di una costa arrotondata lungo tutti i tre quarti distali; arrotondato ne è pure il lato esterno e superiore. Nella parte prossimale la faccia articolare per l'omero, dapprima quasi normale al corpo dell'osso, si ripiega bruscamente allo insù, occupando la faccia interna della parte ascendente del processo olecranico ed assumendo una direzione parallela all'asse generale dell'osso (ciò che già vedemmo nella faccetta accessoria dell'omero). Il processo olecranico poi è sriluppato e robustissimo, è molto dilatato tanto allo insủ che allo ingiù e la porzione ascendente tende a ripiegarsi verso lá faccia interna dell'osso, o meglio verso il corpo dell'animale. Il processo stesso è rotto alla estremità, non è quindi possibile vedere quanto si estendesse allo infuori dell'asse.

L'estremità distale del cubito è essa pure pochissimo espansa; si termina con una faccia normale all'asse dell'osso alla quale aderiva la gran cartilagine contenente le ossa del corpo.

Se non vado errato, troro che fra le Balenottere viventi, quella il cui cubito si accosta di più a quello descritto sia la Balacnoptera rostrata o laticcps.

Ossu delln mano (fig. 32). - Delle ossa della mano abbiamo in tutto: due ossa metacarpali (uno appartiene probabilmente al primo dito, l'altro al secondo o terzo dito) e quattro falangi; non mi sono attentato a determinare sicuramente a qual dito ed a qual numero d'ordine per ciascuu dito appartenessero; tanto però potei redere, che esse presentano tutte lo stesso carattere, di essere cioè molto corte e massiccie ed aver dovuto per conseguenza appartenere ad una mano costrutta sul tipo dell'anzi nominata Balaenoptera rostrata o laticeps.

Bıcino (fig. 33). - Seguendo il Brandt, interpreto siccome corrispondente alla meti del bacino un ossicino di forma irregolare trovato nello scavare lo scheletro di 
Cortandone. Tale ossicino era stato dal Gastaldi fatto disegnare in due posizioni ed i disegni inviati cogli altri al Brandt. La scala in cui furono fatte essendo piccolissima, i dettagli andarono perluti e le due figure ne rimasero quasi simmetriche. Da ciò ne derivò l'errore in cui cadde il Brandt, credendo di avere dinanzi tutte due le metà del bacino e come tali facendole riprodurre nella fig. 32, tav. XXII, delle sue Untersuchungen.

L'osso in questione consta di due parti perfettamente distinguibili, l'una anteriore laminiforme, irregolarmente piana, allungata e ristretta e che sfugge allo indietro nella seconda che è cilindro-compressa e ricurva in modo da sfuggire dal piano verticale in cui trovasi l'asse della prima porzione.

Il Van Beneden figura a tav. IV e V, fig. 28, della classica Ostéographic des Cétacés vivants et fossiles l'osso sinistro del bacino dello scheletro di Balaena mysticetus di Bruxelles e tav. VI, fig. 15, quello pure sinistro della stessa specie dello scheletro di Copenaghen. Il Gaudry nei suoi Enchânements du monde animal dans. les temps gíologiques, vol. I, 1878, a pag. 34, fig. 23, ne rappresenta un terzo destro el appartenente ancora alla stessa specie e dello scheletro del Iuseo di Parigi. Ciascuno di questi ha una forma che lo distingue nettamente ed a prima vista dagli altri. L'osso del bacino di Balaenoptera Gastaldii, comparato a queste tre figure, si trova arere una grandissima analogia di forma e di disposizione delle parti cou quello figurato dal Gaudry del Mfuseo di Parigi; immediatamente poi si riconosce appartenere al lato opposto di quello, cioè al sinistro, e mancare dei due rudimenti ossei rappresentanti il femore e la tibia.

Il Van-Beneden poi, a pagina 84 della stessa Ostéographic, parlando delle ossa del bacino, dice espressamente: "Ces os, d'après leurs rapports ct non d'après lour forme, sont des Ischions $\gg$. Quantunque io non abbia ancora aruta occasione di spogliare lo scheletro di uno di questi animali e quindi mi manchino molte cognizioni per le quali devo continuamente ricorrere a quelle contenute e raccolte nelle opere del Van Beneden, mi sia però lecito esporre un dubbio in me sorto dallo esame della forma di queste ossa, dalla loro posizione rispettivamente allo scheletro restante ed infine dalla posizione dei rudimenti delle estremita per rispetto alle ossa del bacino. Infatti tenendo nella posizione che deve aver naturalmente tenuta la metì sinistra del bacino di Balaenoptera Gustaldii, la parte sua appiattita e laminare sarà collocita orizzontalmente, el al suo angolo posteriore ed esterno si sarì articolata, più o meno mediatamente, la parte prossimale del rudimento di femore e ciò mentre la stessa lamina si prolungava, verso l'angolo posteriore ed interno, in un'apofisi avente una direzione discendente ed allo interno, che andava cosi incontro ad una apofisi simile dell'osso del lato opposto, tendendo a fare con quella e colle porzioni appiattite delle due metà di bacino un cingolo pelvico quasi completo. Non sarebbe egli per conseguenza piü naturale il considerare ciascuna delle metà del bacino, sia della Butcence mysticctus, sia della Balaenoptera Gastaldii, come corrispondente all'osso iliaco ed al pubico insiemo saldati ( 1 ) ed in cui le due parti corrispondenti ai singoli ossi

(1) L'sstme dei rapporti fra le tro ossa componenti ciascuna mela del bacino degli uccelli mi porterebbo ancora alla stessa conclusione.

6 A. Portis. 
fossero limitate dalla posizione del rudimento delle estremità, che non ritenerlo come il solo osso ischiatico di cui non ha nè la forma, nè la posizione rispetto allo scheletro restante, nè la posizione rispetto alla più o meno sviluppata estremità? Mi limito per ora, non potendo dare altre prove, ad enunciare soltanto questa ipotesi; ma essa potrà forse servire come punto di partenza a nuove ricerche sui veri rapporti del bacino dei Cetacei con quello di altri Mammiferi quando fortunati arenamenti di Talassoterii ne forniscano l'occasione.

Dopo aver segnalato tutti i caratteri piủ salienti che potei riscontrare negli aranzi dello scheletro di Cortandone, credo sia sufficientemente dimostrata la necessità di tenere la specie cui esso appartiene distinta dall'altra molto piu comune nell'Alta Italia, dal Plesiocetus o Balcenoptera Cortesii, e di attribuirle un nuovo nome specifico scegliendo a tale scopo quello proposto dallo Strobel come nome di rarietà.

Infine noi non possiamo staccarci dallo scheletro di Cortandone senza segnalare, a proposito di esso, una particolarita che nou la più niente a che fare colla sua sistematica collocazione, ma clie non manca però di attirare tutta la nostra attenzione. Il suo cubito è fortemente intagliato tanto alla faccia esterna che alla interna. I tagli che esso presenta, abbastanza rasti e profondi, furono, per quanto risulta dalla natura loro, evidentemento fatti mentre losso era ancora ricoperto delle parti molli e sono perfettamente analoghi a quelli che si riscontrano sulle ossa dei Balucnotus della Toscana ed evidentemente doruti alla stessa orjgine. In un mio laroro, pubblicato recentemente, ho parlato di questi tagli e di molti altri che dovrò ancora menzionare nel corso di questo mio Catalogo ed loo cercato di dimostrare come essi siano

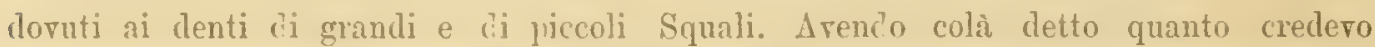
necessario a dimostrare il mio asserto, sarebbe inutile il ripetere qui le stesse ragioni; limitiamoci ad esporre come, secondo lidea altrove accemata, il cadavere della $B a-$ Incnoptera Gastuldii, di cui abbiamo dinanzi alcune parti dello scheletro, fu, prima di venir sepolto e conserrato, addentato dai pesci cani, i quali, nello strappare le carni, intaccurono profondamente lo ossa e ci lasciarono cosi preziose traccie della loro esistenza e delle loro abitudini perfettamente simili a quelle dei loro rappresentanti nostri contemporinci. Le pinne essendo più sottili e sporgenti allo infuri del corpo, yuindi piu facili ad essere abboccate. è molto naturale che contro ad esse siano dapprima rivolti gli sguardi ed i morsi degli assalitori, molto naturale per conseguenza che si presenti abbastanza frequentemente il fatto che noi dobbiamo qui constatare, che cioè mentre le ossa del braccio e dello arambraccio vengono trovate cosi guaste, le altre ossa dello scheletro non presentino che poche o niune traccie di tali aggressioni.

\section{N. 3. Balaenoptera Gastaldii (STropeL).}

Molti anni prima che il Gastaldi scoprisse a Cortandone lo scheletro di Balenottera che veniamo di descrivere, in un'altra localita non ben precisata dell'Astigiana si scoprirono tre vertebre o meglio tre corpi di rertebre di Cetaceo, che rennero conservati nel Museo Mineralogico di Torino. L'illustre Borson le riconobbe per 
tali e di sua mano scrisse una etichetta, ancor oggi aderente all'uno dei tre, che diceva: "Vertebra di un Ceto, semipetrificata, rarissima ». Di poi queste vertebre giacquero per lungo tempo, assieme a molte altre di specie diverse, quasi inosservate, finchè nel 1875 il Van Beneden ne parlò una volta incidentalmente nel suo lavoro: La Butcine fossite thu Musie de Milan (a pag. 751 del Bollettino dell'Accademia di Bruxelles), dicendo: "Le Musée de Minéralogie de Turin possède..... tors vertèbres d'une petite éspèce du même genre (Plcsiocetus) ".

'lutte tre queste vertebre appartengono alla prima metì della regione lombare; fra l'una e le due altre dovettero esserne interposte almeno due che non furono scavate con esse. La vertebra, nel rivo, collocata anteriormente mostra i pedicoli dell'arco neurale forti e molto distanti fra loro in modo da lasciare ancora un ampio canale neurale il cui fondo è piano: le apofisi trasverse nascono molto allo insủ, quasi sugli angoli superiori del corpo, l'una è rotta all'origine, l'altra dopo due centimetri di estensione: quest'nltima mostra un po' li tendenza ad espandersi verso l'estremita; il corpo della vertebra è inferiormente arrotonlato e la ficcia inferiore un po incavata allo ingiro; esso è mancante di tutte e due le epifisi e misura dallo avanti allo indietro $65 \mathrm{~mm}$., verticalmente $73 \mathrm{~mm}$. e trasversalmente $105 \mathrm{~mm}$. Queste due ultime dimensioni prése sulla faccia posteriore.

La seconda rertebra, che abbiamo detto non aver dovuto seguire immediatamente la prima, mostra come le apofisi trasverse nascessero alquanto piu allo ingiù; del resto il suo canale nenrale era pure assai ampio. Il corpo è pure inferiormente arrotondato. a curra però più stretta che per la rertebra precedente, e la superficie inferiore ancora incavata allo ingiro. La sua lunglezza è di $77 \mathrm{~mm}$., il suo diametro verticale ed il trasversale, misurati sulla faccia posteriore, misurano l'uno 74 e l'altro $110 \mathrm{~mm}$. coll'arrertenza che anche questa rertebra manca delle due epifisi.

La terza vertebra presenta gli stessi caratteri delle due precedenti, essa ne però alquanto più grande. Essa mostra la epifisi posteriore staccata bensi ma aderente ancora al corpo per uno straterello di sabbia. L'epifisi è ellittica e grande quasi tanto quanto la faccia epifisaria del corpo di rertebra su cui si appoggiara ( 111 ) stato perfettamente adulto arrebbe dovuto sporgere al di fuori del bordo di quella). La faccia articolare sua è perfettamente piana. La lunghezza del corpo della vertebra, non compresa l'epifisi, è di $76 \mathrm{~mm}$., l'altezza e la larghezza sua, misurate sulla faccia posteriore opposta all'epifisi, toccavano l'una 84, l'altra $113 \mathrm{~mm}$.

I caratteri di queste tre vertebre ci ricorlano quelli della vertebra pure lombare, ma collocata nella serie vertebrale, più allo indietro, che abbiamo descritta parlando dello scheletro di Cortandone. Le loro dimensioni, quantunque in proporzione leggermente maggiori, concordano però assai bene con quelle li cui fu parola parlando dello scheletro suddetto e ci lasciano supporre che le tre vertebre in questione abbiano appartenuto ad un secondo scheletro di Balnenoptera Gastuldii il quale, se avesse raggiunta l'età del primo, lo arrebbe forse di qualche poco superato in volume. Quest'ultimo fatto non è per nulla raro fra gli individui di una stessa specie di Balenottere virenti, tanto meno fra quelli di specie estinte. 


\section{N. 1. Balaenoptera Gastaldii (StrobeL).}

Fra i residui di Cetacei da lungo tempo raccolti nel Museo di Minerulogia della R. Università di Torino ed ora passati a quello di Geologia si trovano tre falangi di Balenottera, di cui una intera e due incomplete. Esse vennero raccolte nell'Astigiana e chal Sismonda F. riconosciute come ossa della mano di una Balenottera; come tali vennero da lui figurate in una tavola inedita che dovera accompagnare la sua descrizione dei Vertebrati fossili piemontesi e le figure distinte coi numeri 3, 4, 5 .

Si rede che esse spettano ad una specie le cui dita sono molte corte e le falangi proporzionalmente allargate, nel quale carattere ed in quello delle dimensioni loro esse andrebbero abbastanza d'accordo con quelle che abbiamo accennate appartenenti alla Balcenoptera Gastuldii, alla quale per ora le riferisco quantunque non mi sia arrischiato di determinare a qual dito esse abbiano appartenuto e nella serie di un dito qual rango abbiano tenuto.

\section{‥ 3. Balaenoptera Gastaldii (STROBEL).}

Rovistando fra i pezzi indeterminati di ossa fossili raccolte dal l'rof. Gastaldi, notai un frammento che subito riconobbi arer fatto parte di un ramo mandibolare di Balcnottera. La località in cui venne scoperto non è data, però il suo modo di fossilizzazione e la natura della roccia che riempie il tronco di canale dentale mi indurrebbero a credere che essa fosse stata rinvenuta nelle argille plioceniche della riviera ligure e probabilmente di Sarona

Il pezzo, lungo 14 centimetri ed alto 7 , appartenne alla parte mediana della mandibola sinistra di un individuo affiatto adulto. Ha la superficie interna pianoconvessa e l'esterna molto convessa, il bordo superiore molto affilato e l'inferiore pure sentito ma piủ tondeggiante, sulla faccia interna si scorge un lievissimo solco scorrente parallelamente al bordo superiore a due millimetri da quello, e sulla fuccia esterna due fori mentonieri. Nelle due sezioni poi dell'osso, si scorge presso al bordo superiore il canale dentale pieno di roccia compatta e durissima formante come un cilindro di quasi un centimetro di diametro. Le dimensioni ed i pochi caratteri del frammento concordano con quelli che abbiamo descritti per la Balacnoptera Gastaldii di Cortandone, e non esito punto ad attribuire questo pezzo alla stessa specie di quella.

Se è vero il fatto che questa porzione di mandibola sia veramente di provenienza dalle argille di Sarona, la Balaenoptera Gastaldii, oltre ad esser stata comune nell'Astigiana, durante l'epoca pliocenica ebbe pure rappresentanti nella odierna Lignria. La nostra ipotesi vien poi resa più probabile dall'essersi trovate sicuramente colà le ossa di cui qui appresso. 


\section{N. 6. Balaenoptera Gastaldii (STROBEL) ?}

Il Prof. Leone Orsini scopriva or è poco tempo nel Rio Torsero presso Albenga una costa, o meglio un frammento di costa di Balenottera lungo un 43 centimetri. Tale fossile appartiene ora al Museo di Mineralogia e Geologia della R. Università di Genova, e mi venne gentilmente comunicato dal Direttore del Museo signor Professore A. Issel.

La forma di questo tronco, mancante tanto della parte prossimale che della distale, ci indica che esso dovette far parte di una delle coste posteriori: esso ha una sezione ellittica verso l'origine ed è appiattito verso l'estremità. Le dimensioni sue ci fanno supporre che abbia appartenuto ad una Balenottera adulta del volume della Balaenioptera Gastaldii o poco più. Segnaliamo quindi una nuova località ligure che ha fornito traccie di Misticeti fossili e probabilmente di Balacnoptera Gastuldii.

\section{N. 7. Balaenoptera Gastaldii (STROBEL)?}

Il Prof. Perez aveva da lungo tempo raccolti e donati al R. Museo Nineralogico di Torino alcuni aranzi di Cetacei fossili scoperti nelle argille delle fornaci di Savona. Sono tre pezzetti irriconoscibili di ossa spugnose, che si puỏ supporre abbiano fatto parte di corpi di vertebra, e diversi piccoli frammenti di coste di giovane individuo. Se realmente queste coste appartennero ad un Misticeto, come i frammenti di ossa corte lasciano ammettere, si trattara qui di un piccolissimo individuo raggiungente appena la metà delle dimensioni della Balacnoptera Gastaldii.

\section{N. 8. Balaenoptera Gastaldii (STROBEL)?}

Fig. 34.

Finalmente faremo ancora menzione di un ultimo frammento di costa di Cetaceo raccolto dal Prof. Gastaldi e del quale non è indicata la località forse perchè parve dapprima non meritasse grande attenzione. Il volume suo ci inclurrebbe a riferire il pezzo alla specie Balaenoptera Gastaldii. Tale frammento ha un certo valore se si considera che esso appartenne alla porzione prossimale di una costa del primo paio e che si mostra decisamente bifido o meglio risultante dalla unione per sorrapposizione di due elementi costali dapprina distinti. Un tale carattere venne già da alcuni Autori invocato persino come distintivo di nuovi generi; è quindi ben naturale che io ne faccia menzione, poichè se realmente il pezzo in questione appartenne ad un individuo di Balcenoptera Gastaldii noi arremmo da aggiungere alla diagnosi della specie anche questa particolarità. 


\section{N. 9. Balaenoptera (Plesiocetus) Cortesii (Desu.).}

FıG. $35-66,76$.

\section{LA BALENOTTERA DI MONTAFIA.}

Nel 1874 il Prof. Gastaldi, dopo aver ricevuto in diverse riprese vertebre di Cetaceo provenienti da Montafia, conobbe la località precisa in cui erano state trovate e vi fece eseguire un ampio scavo che gli procurò : il cranio, un rilevante numero di vertebre a cominciare dallo atlante, una scrpola ed un numero considerevole di coste di una piccola Balenottera.

Dopo avere per molti mesi e con una pazienza ed abilita ammirabili dato opera alla ristaurazione del fossile, ne fece un cenno in un'appendice della Gazzctta Pirmontese sotto il titolo: Uno scheretro di Balena a MLontafia, che fu poi anche stampato in fascicolo a parte. In tale cenno vien soltanto detto che il fossile appartiene al genere Cetotherium, mentre più copiosi sono i dettagli riguardanti i terreni superiori della ralle del Po ed il giacimento del fossile.

Forse il Gastaldi si proponeva di descriverlo piu dettagliatamente in seguito, e ne fu distolto da altri lavori; il fatto sta intanto che una illustrazione di questo importante animale è ancora al presente un desiderio al quale cercherò di soddisfare del mio meglio.

Crunio (fig. $35,36,37,38$ ). - Il cranio è assai bene conservato: esso consti oggigiomo di 6 pezzi: la parte superiore o scatola vertebrale, il vomere, $\mathrm{i}$ due intermascellari nssai guasti ed $\mathrm{i}$ due mascellari relativamente completi. Dall'esame dello insieme possiamo rilevare come il cranio fosse lunghissmo $(2,25 \mathrm{~m}$.$) , diritto, molto$ depresso e strettissimo non misurando dalla ficcia esterna di un arco zigomatico a quella dell'altro che $77 \mathrm{~cm}$.

Occipiulule. - Se poi ci facciamo a stuliarne i dettagli, osserviamo per primi - cosa la posizione dei condili occipitali collocati molto all'insù, per modo che cssi quasi restano nella faccia superiore del cranio ed il foro occipitale viene pure ar arere un uscita guardante all'indietro ed all'insù. Perció, come osserva il Van Beneden a proposito dello scheletro del Iruseo di Milano, l'asse del cranio non poteva essere sul prolungamento di quello della colonna vertebrale, ma fare con esso un angolo ottnso coll'apertura rivolta all'insù.

Abbiamo già visto come nel cranio della Balcenoptera Gustuldii i punti estremi posteriori non si trovino sulla faccia articolare dei condili, ma invece sugli angoli posternesterni del cranio, quasi in coincidenza colla sutura tra gli occipitali esterni ed i tëmporali; qui succede lo stesso fatto; i condili occipitali paiono spinti allo innanzi della linea posteriore del cranio, la quale riesce per conseguenza tutta ondulata e faciente due profonde insenature allo avanti, una da ciascun lato del foro occipitale e compresa fra il condilo e l'angolo esterno di ciascuno ex-occipitale. Queste due insenature si fanno sentire poi come lievi depressioni laterali anche sulla faccia superiore dell'occipitale stesso. 
La squama occipitale poi, gia pienamente fusa cogli ex-occipitali, partendo dal margine posteriore del cranio e dal foro occipitale con una superficie esterna depressissimamente convessa sulla linea meliana ed ondulata ai fianchi, raggiunge in breve la sommità della volta craniana ed allora corre orizzontalmente sulla linea mediana. ed un po' rigonfia ai lati per quasi tutta la sua estensione, salvo a discendere poi leggermente all'ingiù verso l'origine delle ossa nasali alla sua estremità anteriore. Lissa riesce cosi ad arere una forma triangolare molto acuta i cui bordi laterali coincidono colle suture occipito-temporali all'indietro, ed occipito-parietali allo avanti e sporgenti infuori dalle faccie interne delle fosse temporali, mascherando in parte queste ultime allorchè il cranio venga guardato dalla faccia superiore.

Comparando, per ciò che riguarda l'occipitale, il cranio di Montafia con quello del Cortesi 1806 o del Museo di Milano, noi vediamo come la forma dell'osso sia ad un dipresso la medesima; osserviamo soltanto che l'angolo anteriore dell'occipitale è più aguzzo nel cranio di Montafia e le insenature più pronunziate, che quindi l'osso è in questo esemplare proporzionatamente più esteso in lunghezza e meno in larghezza.

Temporali. - Le ossa temporali molto massiccie hanno ciascuna, la loro apofisi zigomatica molto ricurva, diretta molto allo avanti ed un pochino allo infuori, terminata in punta all'estremità che gurrda il margine posteriore esterno dell'osso frontale, non riescendo però a toccarlo, perchè la direzione loro le porterebbe molto piu in basso.

L'apofisi zigomatica del cranio del Cortesi 1806 i invece molto piu ricurva allo infuori e meno allo avanti, è meno decisamente appuntita e si termina piu lontano, allo indietro del bordo posteriore dell'osso frontale.

In complesso le ossa temporali cli quel cranio rendono più saliente il carattere giì notato per l'occipitale, di essere cioè più corto e più csteso posteriormente in larghezza che non quello di Montafia 1874.

Parieluli. - Le ossa parietali, nascoste sotto le sporgenze laterali dell'occipitale, son ridotte ad una zona bassa che forma la parete interua e verticale di ciascuna fossa temporale. Esse si inacutiscono rapidamente allo innanzi e si congimgono sulla linea meiliana con una zona superiore, contornante l'angolo anteriore delloccipitale, lunga un paio di centimetri e nascondentesi allo indietro sotto l'angolo stesso che è ¡ui alquanto corroso. Non riconosciamo qui traccia di quella zona interparietale cosi ben definita nel cranio della Balacnoptera Gastuddii.

La posizione e forma delle ossa parietali nel cranio di Montafia 1874 va, per quanto mi è dato credere, sufficientemente d'accordo con quella del cranio Cortesi 1806.

Fromllli. - Le ossa frontali molte respinte allo avanti e ricoperte dalle parietali, non possono venir ben descritte, perchè molto difettose nella parte anteriore. Conpletissime ne sono invece le apofisi espanse orbitali, il cui margine posteriore è diretto affitto allo esterno ficendo un angolo più che retto coll'asse longitudinale del cranio, inquantochè la sua direzione oltre ad essere trastersale è pure lievemente marcata allo indietro. Nel cranio dello scheletro Cortesi 1806 , il margine posteriore di ciascun processo orbitale è invece nella sua prima metà diretto allo infuori e lievemente allo avanti, nella metà esterna diretto decisamente allo infuori.

Dalle differenze notate nelle ossa fin qui accennate nei due cranii ne viene che la porzione di fossa temporale che si mostra a giorno a chi guarda il cranio dal di 
sopra $\dot{e}$ in quello di Montafia più angolosa, più lunga e più stretta, mentre in quello del Museo di Milano essa ha un profilo più arrotondato, è più corta e più espansa.

L'arcata orbitale è nel cranio di Montafia molto ampia e regolare, il canale del nervo ottico ha una direzione obliqua allo esterno ed allo avanti: in ciò si accorda con quanto è visibile nel cranio del Iuseo di Milano.

Nusuli. - Mancano al cranio di Montafia quasi completamente le ossa nasali; dorevano però queste essere molto allungate allo indietro, a quanto almeno si può scorgere dalle loro radici posteriori ancora in posto, e dovevano essere contornate lateralmente per quasi tutta la loro lunghezza dalle sottili apofisi posteriori degli intermascellari, delle quali pure ancora si riscontrano le traccie di sutura.

Intermascellari. - Il corpo delle ossa intermascellari al dinanzi della fossa nasale era subcilindrico posteriormente, appiattito anteriormente e lievemente espanso presso al borlo libero anteriore, a differenza di quanto si osserva per le stesse ossa nel cranio Cortesi 1806 dove esse conservano presso a poco la stessa forma cominciando dal limite anteriore della fossa nasale fino al loro borlo libero anteriore.

Huscellari. - Le ossa mascellari non presentano, a vederle così isolate, altre noteroli differenze dalle corrispondenti del cranio Cortesi, che di essere decisamente pii strette ed appuntite tanto sulla faccia inferiore, quanto, e maggiormente, sulla faccia superiore. Pare che allo indietro portassero, dinanzi al bordo anteriore dei frontali (parte espansa esterna), quella stessa apofisi che il Van Beneden fa notare sul cranio di Milano e che dice caratteristica delle vere Balene.

Vomrie. - Il vomere del cranio di Montafia è ridotto ad un frammento di $95 \mathrm{~cm}$. di lunghezza e possiamo, come del resto anche dalle ossa mascellari, ricavarne che il rostro dovette essere lunghissimo, stretto e non presentare alcuna traccia di curvatura allo ingiiu; dorette per conseguenza. come quello del Cortesi 1806 , non essere fornito che di cortissimi fanoni.

Cinsal limpanic' (fig. 39, 40,41). - Assieme allo sclieletro di llontafia veme trovata eziandio la cassa auditiva sinistra non in perfetto stato di conservazione, è vero, ma tale però da farci sufficientemente conoscere la forma di quest'organo. Essa è stretta, allungata, con un'apertura molto ampia e col bordo inferiore compresso. Nou somiglia punto a quella della Balacnoptera Gastaldii che abbiamo descritta, nè alcuna relazione presenta con quella attaccata al teschio del Museo di Milano, nè con quelli disegnata dal Capellini ed appartenente allo schıletro del MLuseo di Bologna. La sua forma ed i suoi caratteri ci licono esser quella una cassa auditiva di vera Balenottera o piuttosto di Burtinopsis, poichè trovo in essa e sovratutto nel profilo della parte posteriore e nell'apertura della spira della parete interna molta amalogia colle casse auditive del Burtinopsis similis figurate dal Van Beneden.

Inudibole' (fig. 42, 43, 44, 45, 46, 47). - Questo eminente Cetologo dì poi, ed a ragione, somma importanza, nella distinzione dei generi e delle specie di Misticeti, alla forma e ai caratteri delle mandibole. Il cranio di Montafia possiede entrambe e completissime queste ossa; trovasi per conseguenza, sotto un tal punto di vista, particolarmente favorito. La lunghezza di ciascun ramo mandibolare misurata secondo la curra tocca i metri 2,10 ; la corda tirata dal margine anteriore del foro d imbocco pel canale dentale all'estremitå anteriore del ramo misura m. 1,79; la massima saetta com- 
presa fra questa corla e la faccia interna della mandibola misura $79 \mathrm{~mm}$. di lunghezza: si vede adunque quale enorme differenza esista a questo riguardo fra le mandibole della Balacnoptera Gastaldii e quelle dello scheletro di Montafia e quanto più queste si assomiglino alle mandibole dei Musei di Milano, di Bologna e di Parma (Cortesi 1816).

11 ramo mandibolare di ciascuna parte non presenta, se ne eccettui leggerissima traccia nell'estrema porzione anteriore, alcuna distorsione per cui la sua faccia interna tenda alla estremitì anteriore a divenir superiore; in ciò si assomiglia ancora a quelle degli scheletri sopramenzionati.

Cosi pure l'altezza del ramo mandibolare si conserva con lievissime differenze sempre uguale, dalla regione in cui ha origine il canale dentale sino alla estremita, mostrando di uon decrescere allo aranti. Le due faccie interna ed esterna sono l'una dall'altra separate dai due bordi superiore ed inferiore, tutti e due pochissimo sporgenti nella regione posteriore, molto piu sentiti invece nell'anterior?.

La superficie interna si conserva sempre ilebolmente convessa per i tre quarti posteriori del ramo mandibolare divenenendo poi quasi piana nel quarto anteriore, mentre la superficie esterna comincia ad essere fortemente convessa subito al davanti del contilo e si conserva tale pel quasi tutta la lunghezza del ramo, non scemando che lentissimamente di convessità nel quinto anteriore di ciascun ramo.

Se, come già facemmo per la Balacnoptera Gastaldii, passiamo ora in rivisti le singule parti, noi osserviamo prima di tutto la forma el $\mathrm{i}$ rapporti del condilo che meritano infatti tutta la nostra attenzione.

La mandibola non è in questa regione che pochissimo ingrossata di fianco (alquanto e superiormente dal lato interno e pochissimo dal lato esterno); è invece alquanto piu elevata del resto e la faccia condiloidea è in pieno collocata posteriormente con insensibile inclinazione allo insù. Essa occupa i due terzi superiori della faccia posteriore dell'osso e si perde insensibilinente nel terzo inferiore o Trullone un po' piu sporgente all'inclietro.

Il condilo è un po' meglio isolato dal tallone quando lo si osservi dai fianchi; si vede allora all'esterno una lieve depressione, che si perde subito verso l'avanti e che separa nettamente le due parti nominate, ed all'interno un'altra depressione corrispondente ua ancol meno affondata e piu ampia, lungo la quale scorrevano i tronclis nervosi e sangnigni che imboccavano il canale dentale posto jiì aranti sul prolungamento di questa depressione.

L'intaglio sigmoide è nella mandibola di questa specie di una grandissima semplicità. L̀ lunghissimo ed il suo fondo è orizzontale e linitato posteriormente dal piccolo rilievo condiloideo ed all'avanti dalla apofisi coronoide appiattita e ripiegata all'infuori e che, pure essendo assai piccola relativamente al volume dell'osso, non manca però di avere un certo sviluppo, tanto maggiore poi se la si osserva in coufronto con quella della Balaenoptera Gastaldii.

La posizione del forame d'ingresso al canale dentale è poi affatto caratteristica per questa specie. Mentre tale imbocco è nelle altre specie di Balenottere viventi o fossili quasi sempre collocato sulla faccia interna della mandibola ma più o meno guardante allo insù ed in modo da interessare il fondo dello intaglio sigmoideo, in

7 A. Portis. 
questa specie esso guarda direttamente allo indentro mantenendosi a metà altezza del ramo mandibolare e parallelo all'asse del medesimo.

Altro fatto degno di nota è che questo imbocca è collocato molto più allo avanti che nelle altre specie, per cui $i$ tronchi nerrosi e sanguigni debbono fare un lungo tratto allo scoperto prima di immettervisi, e come se ciò non bastasse, dopo essersi affondato nella superlicie interna del ramo, il canale rimane ancora per un tratto di 5 a 6 centimetri collo aspetto di canale beante senza acquistare una parete propria anche verso l'interno che allorquando ha superata la porzione diritta posteriore della mandibola eil è entrato, verticalmente al disctto dell'apofisi coronoide, nella porzione ricurva di essa.

11 canale dentale occupa poi nello interno del ramo mandibolare il terzo superiore dell'altezza di esso. e si mostra in qualche punto ripieno di roccia sabbiosa, ed in qualche altro, vuoto o rivestito di piccoli cristalli di calcite. Lsso si manifesta alla superficie dell'osso, verso la faccia interna per una serie di piccoli fori diretti allo avanti e che finiscono, assieme, per simulare un lungo solco pochissimo aftondato e scorrente parallelamente ed alla distanza di pochi millimetri al bordo superiore del ramo manlibolare; verso la faccia esterna per mezzo dei grandi e frequenti fori mentonieri, di cui ne potei contare undici sulla mandibola sinistra senza essere certo che non re ne siano stati di piu.

Allo avanti il canale dentale si fa superficiale, presso alla estremità della mandibola esso ne occupa il bordo superiore e, giunto alla faccia anteriore dell'osso, discende verticalmente e si ripiega in seguito allo esterno in modo analogo a quanto vedemmo nella Bataenoptera Gastaldii ed a quanto si osserva nel Burtinopsis similis V. Ben. Nella porzione anteriore di ciascun ramo mandibolare scorgesi inoltre sulla faccia interna ed al disotto della metà altezza di questa un secondo solco molto accentuato alla estremità anteriore, che va rapidamente perdendosi allo indietro e la cui direzione è parallela all'asse della mandibola. Anche questo solco abbiamo veduto comportarsi presso a poco nello stesso modo nella Balnenoptera Gastaldii e lo vediamo ancora quasi tale nel Burtinopsis similis di Anversa.

La mandibola del cranio di Montafia 1874 concorda nella sua forma e nei suoi caratteri speciali abbastanza con quella del Museo di Milano (Cortesi 1806); ha perỏ il condilo più sviluppato in altezza e meno in larghezza, mentre la depressione separante il condilo dal tallone è, nolla nostra specie, meno pronunziata.

In conclusione, i caratteri della mandibola dello scheletro di Montafia, nonchè quelli tratti dalla sua cassa auditiva ci indurrebbero assai più a collocare nel genere Burtinopsis che non nel genere Balacnoptera il fossile di cui si tratta, tanto piu allorchè si abbia riguardo ancora alla forma delle vertebre cervicali anteriori di cui è ora tempo di far pirola.

Verlebre. - Della regione cervicale ci sono state conservate sei rertebre, le cinque prime cioè e la settima; la sesta sventuratamente andó perduta.

Allanle (fig. 48, 49,50). - Liatlante, un po' guasto alla sommità, ci mostra invece molto bene la forma delle faccie articolari e del foro vertebrale. Esso è notevole per il suo spassore, misurando inferiormente ben 7 centimetri di diametro antero-posteriore; il suo diametro trasverso massimo, comprese le apofisi trasverse, ̀̀ 
di $265 \mathrm{~mm}$.; senza le apofisi misura dal bordo esterno di una delle faccie articolari posteriori a quello dell'altra $215 \mathrm{~mm}$. Il suo diametro verticale dorera toccare i $185 \mathrm{~mm}$.

Le due faccie articolari anteriori sono amplissime, debolmente ed uniformemente incarate e convergenti in basso, dove non sono separate che da un legrierissimo e strettissimo solco verticale, tantochè a primo aspetto paiono confondersi in una sola.

Le faccie posteriori abbastanza ampie, benchè in minor grado delle anteriori. sono piano-convesse, lievemente inclinate allo infuori e separate in basso l'una dall'altra đa un ampissimo tratto contro al quale si appoggia l'apofisi odontoide dell'asse. Il forame vertebrale è poi altissimo, molto allargato nella metà sua superiore, ed invece molto piu ristretto nella inferiore che si termina in basso, non giì arl angolo od a fessura, ma con bordo inferiore a stretta curva.

Finalmente le apofisi trasverse, pochissimo estese longitudinalmente e trasrersalmente. lo sono invece di molto verticalmente, costituendo delle lamine collocate normalmente all'asse dell'animale sulle faccie latero-superiori dello atlante e presso al loro bordo posteriore.

L'atlante dello scheletro di Jontafia rassomiglia alquanto a quello della Balacnoptera rostratella di Anversa, ma molto di più ancora a quello del Burtinopsis simitis V. BEx. raffigurato a tav. 91 degli Ossements fossiles d'Anvers, non differendone che per la parte inferiore del foro vertebrale un po' meno ristretta e per le apofisi trasterse più deboli.

Che l'atlante delle Balenottere italiane rassomigliasse assai a quello del genere Burtinupsis l'areva già notato anche il Van Beneden, il quale a pag. 81 del terzo fascicolo della sua Descrizione degli ossami fossiti di Anversa, piu volte citato, dice: "Si l'on compare cetatlas (del Burtinopsis minutus) arec le même os de la Balénoptère fossile d'Italic (Balacnoptera Cortesii) dont les specimens sont conserrés à Milan, à Turin et ì Parme, on troure entre enx une grande ressemblance sous ce rapport (dans le rétrécissement du trou vertébral par l'efłet de l’âge) ». Parmi soltinto che le analogie della Balenottera italiana, sotto il rapporto della forma dello atlante. siano piu eridenti col Burtinopsis similis che non col Burtinopsis mimutus.

Asse (fig. 51, 52). - Il corpo della vertelra asse è per lo meno altrettanto potente che quello dell'atlante, il suo arco neurale è molto sviluppato e l'apofisi spinosa. che pure dovera essere assai sviluppata ma che è ora rotta, era molto inclinata allo inclietro; inclinate allo indietro sono pure le apofisi trasvierse superiori nascenti dal basso dell'arco neurale; e le apofisi trisverse inferiori nascenti dal basso del corpo della vertebra, esse pure rivolte allo infnori ed allo indietro, hamo uno sviluppo di poco superiore di quello delle superiori. Le due faccie articolari anteriori, piano-coneave ed inclinate allo indentro, si uniscono mutuamente al di sotto dell'apofisi odontoile che è qui assai sviluppata in ampiezza, pochissimo in potenza. Il camale neurale ampissimo, quasi altrettanto elerato quanto largo.

Anche la vertebra asse presenta analogie colla stessa vertebra del Burtinopsis similis sia nell'ampiezza delle faccie articolari anteriori, sia nello sriluppo dell'apofisi odontoide, sia nella forma generale delle parti conservate; ne differisce soltanto nello șiluppo delle apofisi trasverse inferiori molto piu forti in quella specie di Burtinopsis 
che nella nostra Balenottera: è anzi probabile che in questa le trasverse inferiori non potessero colla loro espansione diretta allo insù raggiungere le superiori e formare cosí un anello completo da ciascun lato del corpo della vertebra.

I'ertebre cervicali (fig. $53,54,55$ ). - Le vertebre cervicali che succedono all'asse hanno cjascuna il corpo il cui spessore è allo incirca la metà di quello dell'asse o dell'atlante. La forma di ciascun corpo di rertebra è nettamente quadrangolare con grande predominio del diametro trasversale sul verticale (a differenza di quanto vedemmo per la Balacnoptera Gastaldii) e cogli angoli leggermente arrotondati. I loro archi vertebrali sono ampissimi, poco robusti e depressi; le apofisi trasverse nascenti dalla origine delli archi e più forti delle inferiori; queste hen sviluppate e visibili per le vertebre $3^{\mathrm{a}}, 4^{\mathrm{a}}, 5^{\mathrm{a}}$, ridotte forse a piccoli tubercoli nella $6^{\mathrm{a}}$ e mancanti assolutamente uella $7^{\mathrm{a}}$. Le loro grandi faccie articolari quasi perfettamente piane, tanto le anteteriori cle le posteriori.

La settima vertebra cerricale manca dell'epifisi posteriore; ciò ci dimostra che, benchè abbiamo a che fare con un individuo adulto, esso non era però stravecchio allorchè venne a morte.

Verlebre dorsali (fig. 56, 57). - Delle vertebre dorsali ne vennero conservate dieci: la $1^{\mathrm{a}}-3^{\mathrm{a}}$, la $5^{\mathrm{a}}-11^{\mathrm{a}}$. 11 corpo della prima è ancora quasi tanto sottile quanto quello delle ultime cervicali, vanno però le seguenti gradatamente aumentando in lunghezza. Così pure cambia rapidamente dalla prima all'ultima la forma del corpo della rertebra, inquantochè nelle prime è ancora quasi rettangolare, molto più largo che alto, siccome osservammo per le cervicali (che anzi nella quinta dorsale la faccia articolare posteriore si mostra addirittura trapezoidale col maggior lato trasversale ed in hasso e ciò per lo sriluppo di tuhercoli accessorii sopportanti le faccette laterali di articolazione coi capitoli delle coste); le seguenti invece vanno via via perdendo gli angoli inferiori e finiscono per non arere piu (in luogo di una faccia inferiore e delle due latcrali) che una faccia sola inferiore, la quale si estende in curva regolare pei fianchi sino all'origine delle apofisi trasverse.

Sui fianchi dei corpi delle rertebre $2^{a}, 3^{3}, 5^{2}, 6^{a}$ e presso alla faccia posteriore sono molto ben visibili i punti di attacco per l'articolazione diretta o meno del capitolo delle coste; sappiamo per conseguenza che almeno le sei coste anteriori da ciascuna parte arevano aderenza, oltrechè per il tubercolo colla vertebra loro corrispondente, ancora pel capitolo colla vertebra antecedente.

Il canale neurale si conserva in tutta la regione dorsale ampissimo, molto depresso, lieremente angoloso in alto ed a fondo piano ed orizzontale. Gli archi neurali sono delicati e debolissimi nella regione dorsale anteriore e ripiegati allo indietro: aumentano rapiclamente di volume e di forza nella regione posteriore dove sono invece molto sriluppati. Lo stesso dicasi delle apofisi spinose, dapprima piccolissime, sottili, non sporgenti pủ di un centimetro dalla sommità dell'arco e dirette verticalmente allo insù; quindi molto più forti, alte almeno una diecina di centimetri ed inclinate allo indietro.

Di pari passo colle apofisi spinose si sviluppano le apofisi accessorie od articolari, sottili ed appiattite alla seconda dorsale e colla faccia articolare rivolta allo insú; alla ottava dorsale sono due grossi tubercoli, grandi quasi tanto quanto le 
apofisi trasverse e dirette orizzontalmente allo avanti colle faccette articolari rivolte allo ingiù, mentre alla undecima esse si sono ancora una rolta modificate pigliando la posizione che occuperanno per tutta la regione lombare, cioè collocate piủ presso la linea mediana e mostrandosi compresse da lato e colle faccette articolari inclinate allo indentro verso la linea mediana stessa.

Le apofisi trasverse, sempre piủ sottili quanto più le vertebre cui appartengono sono anteriori, nascono nelle vertebre della regione anteriore direttamente dai fianchi dell'urco neurale; nella clecima ed undecima dagli angoli superiori del corpo assieme ai pedicoli dell' arco stesso. P'er le vertebre prima a settima sono dirette allo infuori e sempre più leggermente allo insủ; per la ottava e seguenti sono dirette orizzontalmente el anclee un pochino inclinate in basso. Tutte hanno una abbastanza costante lunghezza dall'origine all'estremità, sviluppandosi per un sei o sette centimetri dall'origine; presso alla estremità si dilatano alquanto, sorratutto le anteriori, e portano una vasta faccetta per l'articolazione della costa.

C'osle (fig. 58, 59, 60, 61). - Abbiamo un grandissimo numero di frammenti di coste or più or meno considerevoli: fra le altre una meta prossimale di una prima costa di sinistra con mólto ben conservati tubercolo e capitolo. In generale le coste della regione anteriore sono appiattite per tutta la loro lunghezza, mentre quelle della regione posteriore si mostruno in generale irregolarmente cilindriche nella meta superiore e pochissimo appiattite nella inferiore. Alcune coste furono rotte clurante la vita dello animale, si risaldarono poi piu o meno perfettamente, ma le ossa che subirono tali vicende rimasero distorte od interrotte da rigonfiamenti ossei talora considerevoli.

Vertebre lombari (fig. 62, 63). - Inella regione lombare ci sono rimaste undici vertebre non consecutire. Sei sono ridotte ai soli corpi molto guasti ed imperfetti; le altre cinque, le anteriori, meglio conservate, mostrano il loro corpo carenato sulla faccia inferiore e dal quale sporgono nella meta superiore di ciascun fianco le apofisi trasverse non molto dilatate alla estremita, appiattite e molto luughe e gli archi neurali conserranti (relativamente alla regione cui appartengono) i caratteri che abbiamo notati per le vertebre dorsali, di essere cioè molto ampi e bassi, circoscrivendo cosi un canale neurale largo e depresso; sono del resto molto forti e robusti e darano origine ad una apofisi spinosa lunga (nessuna è completa fino all'estremità), forte ed inclinata allo indietro. Le apofisi articolari sono qui molto ridotte in volume, respinte alla sommità degli arclıi e colle faccette articolari inclinate allo indentro ed allo innanzi.

Finalmente allo stesso modo in cui arvertimmo che l'ultima cervicale mancava della epifisi posteriore, occorre ancora notare come delle rertebre dorsali e lombari che abbiamo linanzi. alcune manchino or dell'una or dell'altra or di entrambe le cpifisi, mentre altre vertebre, pur arendo conservate entrambe le epifisi, mostrano come non fossero ancora a quelle perfettamente saldate. Senza insistere troppo su questo fatto, ¿̇ però bene averlo sempre presente, poichè esso ci dimostra che il nostro individuo di IIontafia, quantunque abbia dimensioni per lo meno uguali a quelle dello scheletro della Balenottera di Milano e superiori a quelle degli scheletri di Parma, non era tuttavia uno degli individui recchissimi ed era per conseguenza suscettibile ancora di veder cresciute le proprie dimensioni. 
Scapola (fig. 64, 65, 66). - Una delle parti che nello stato odierno della scienza ha importanza grandissima nella determinazione dei generi e delle specie di Cetacei è certamente la scapola. Per noi italiani ha poi un interesse tanto più grande inquantochè la mutilazione e la successira troppo completa restaurazione della scapola dell'esemplare di Jilano diede luogo ad errori ed a dubbiezze allorche si trattò di determinare con sicurezza il genere cui esso apparteneva, gli uni ammettendo, negando gli altri che quella aresse mai posseduta un'apofisi coracoidea. Quantunque il Van Beneden abbia cercato di vincere queste dubbiezze dimostrando come la scapola in questione fu mutilata in corrispondenza della origine di tale apofisi, tuttavia era ben desiderabile che un altro individuo renisse rinvenuto, il quale fosse fornito di una scapola abbastanza ben conservata da poter togliere ogni resto di dubbio a chi non fosse ancora perfettamente tranquillo a tale riguardo.

Egli è per ciỏ che io salutai con gioia lo scheletro di Montafia il quale mi forniva appunto quest'osso. Infatti, quantunque diversi scheletri di Balenottere fossili esistano nel Museo di Parma, noi non abbiaino colà che due scapole, quella di Montefalcone e quella di Castellarquato, la di cui forma è troppo diversa da quella del Museo di Milano perchè ce ne possiamo serrire in sostituzione delle imperfezioni di quella; mancante di scapola trovasi pure lo scheletro del Museo di Bologna e fra le parti della Baluenoptera Gastaldii che reniamo di descrivere mancano pure le scapole. Lo sclseletro di Montafia rimedia a queste mancanze, sopprime intieramente $\mathrm{i}$ dubbi, poichẻ in essa, ad una forma che siacorda con quella della scapola delloriginale Cortesi 1806 , si unisce la presenza di un processo coracoideo e di un acromio sriluppati e riconoscibili.

La scapola destra di Montafia è, come quella del ILuseo di IIilano, molto estesa nel senso antero-posteriore, molto meno nel senso verticale: nelle sua regione mediana è sottilissima, il suo contorno consta di sei lati molto ben definiti e distinti: uno superiore quasi rettilineo ed il più lungo di tutti, uno antero-superiore corto e leggermente ricurvo, uno antero-inferiore rettilineo ed obliquo rerso l'indietro e l'ingiù, linferiore, che non è altro che la fiscia articolare per l'onero, quasi piana, uno infero-posteriore rettilineo e lunghissimo, uno finalmente postero-superiore di nuovo corto e leggermente ricurvo. I margini postero-superiore, superiore $c$ superoanteriore si possono benissimo consilerare come uno solo, al quale, dalle tracce ancora visibili su di esso, dovera aderire una grandissima cartilagine ampliante il campo della scapola ed a scapito della quale quest'ultima era renuta man mano estendendosi.

Il margine anteriore è, allorchè si osserri la scapola dal lato esterno, quasi completamente mascherato dal margine spinale e questo nella metì inferiore dà $\operatorname{luog}(r$ ad un processo coracoideo molto sviluppato e compresso ai lati, il quale sgraziatamente è rotto ad un paio di centinetri dalla sua origine: per noi è intanto importante l'averlo constatato. Similmente sull'angolo anteriore, sull'angolo e come continuazione del margine interno della faccia articolare per l'omero redesi l'origine del tozzo acromio, il quale è esso pure rotto poco allo innanzi della sta origine e non ci permette quindi di osserrare di quanto si sviluppasse allo avanti.

Quando arrò detto che la faccia interna della scapola $\dot{e}$ concava e la esterna è piana, arrò finito di enumerare i caratteri di quest'osso per mezzo del quale ci 
siamo assicurati che il Plesiocetus Cortesii non è. altro che una Balenottera e cho il genere Cetotheriophanes sorr'esso fondato non può venir conservato.

Falunge (fig. 76). - Ci maucano dello scheletro di Montafia tutte le altre ossa delle estremità (alcune però le, vedremo trattando successivamente di altri individui piemontesi della stessa specie), all'infuori di un ossicino della mano. $\grave{\mathrm{L}}$ probabilmente una delle falangi estreme e forse del primo dito; vediamo da quella come nello individuo di Jontafia le mani dovettero essere, come generalmente nelle Balenottere, relativamente corte, ciò che del resto abbiamo pure già osservato precedentemente di un'altra specie piemontese, della Balaenoptera Gastaldii.

\section{N. 11. Plesiocetus (Balaenoptera) Cortesii (DesM.).}

LA BALENOTTERA DI BAGNASCO.

Il Museo di Torino possiede gli avanzi di Cetaceo fossile raccolti a Bagnasco dal Borson e da lui illustrati nel 1830 (V. Bibliogr.. $\mathrm{n}^{0}$ 51).

I pezzi principali provenienti da questa scoperta sono:

a) Un frammento del cranio mostrante la scatola cerebrale:

b) Un secondo frammento del cranio mostrante parte del romere e dei mascellari superiori, nonchè gran parte delle mandilole:

c) Un gruppo di vertebre dorsali (regione anteriore) cui sono aderenti frammenti di coste (porzione prossinale):

d) Un gruppo di vertebre (sette lombari) :

c) Un gruppo di frammenti di coste saldamente impastate fra loro e colla materia rocciosa che le arviluppa:

f), g), ecc. Frammenti di vertebre, di corpi di rertebre, di coste, piủ o meno arrolti nella roccia.

I pezzi (a) é b) furono descritti e figurati dal Borson, il quale riconobbe che essi, come i seguenti, appartener dorettero arl un qualche Cetaceo e disse, parlando dell'uno, $)$ : "En parcourant le sarant ourrage: Recherches sur les ossements "fossilrs, je trouve au tome $V$, partie $1^{\text {re }}$, que la portion de machoire en question " peut avoir quelque rapport arec la fig. 2 de la planche XX゙T qu'est 1/10 de la " grandeur de loriginal, et que l'illustre auteur dit à page 308 (1) être la tête " adulte de la Baleine proprement dite du Cap de Bonne-Espèrance. Au reste, par « les restiges si incomplètes et si mutilés que nous avons, je ne puis donner ce rap" port que pour un suupçon même rague ".

Intorno all'altro frammento, a), cosi si esprimeva il Borson: "N'ayant rien " trouvé, parmi les nombreux ossements décrits dans le célébre ouvrage plusieurs " fois cité, qui eut rquelque rapport arec l'os en question, je pris le parti d'en en-

(1) Mi occorre di far" iwtare come essendo io andato a cercare nell'opera citata del Cuvier la figura indicata (per aver io a mano la quarta edizione, le indicazioni del Borson cortisponderebbero a vol. VIll, parte 24 , pag. 239 del testo e tav. $220 \hat{0}$, fig. 2 dell'atlante), trovo che il paragone non fus fatto colla testa adulta della Balena del Capo, ma bensi con quella della giovane Balena del Capo, essendo questa data ad '1o nella fiyura 2, mentre quella \& data ad un $1 / \%$ nello figure $5-9$ della stessa tavola. 
* voyer à son illustre anteur une copie en plâtre. $M^{r}$ le Baron Cuvier eut la com-

* plaisance de m’ẻcrire, après quelques détails anatomiques, que cet os appartenait

« très probablement à un Cétacé; mais que, avant tout, il fallait le dégager de

" tout le sable et de toute la terre qui l'incrustajent. C"est ce que j'ai fait arec

* tout le soin possible; mais on a vu que d'un côté la fragilité de l'os et de l'autre

* l'extrème résistence de cette sorte de marbre, dont les cavités intérieures sont

« renplies, sont un obstacle à le remettre complètement dans son premier état. Je

" désire fort que les dessins que j'en ai fait arec toute l'éxactitude qu'il m'a été

« possible d'y employer mettent les naturalistes dans le cas de déterminer à quel ani-

" mal de l'ancien monde cet os a appartenu ».

Questo ultimo c'esiderio espresso dal Borson doveva rimanere insoddisfatto poichè i suoi disegni, quantunque dal lato materiale perfettamente eseguiti, rennero condotti in guisa tanto schematica (come ad esempio la punteggiatura per indicare dove l'osso è rotto) che $\mathrm{i}$ caratteri adatti ad un riconoscimento anatomico del frammento sparvero affatto; e perchè il Borson si era lasciato forse troppo lungi condurre ad immaginare un tipo affatto nuovo, credette forse trattarsi di aperture naturali là dove non era questione che di semplice sfondamento delle ossa avvenuto posteriormente e considerò come rotture di ossa dei margini naturali un po' guasti e consunti. Per tali motivi ne arvenne che la punteggiatura è molto soventi fuor di luogo, e chi ispezioni semplicemente le figure non potrå che difficilmente farsi un'ilea esatta delle parti che gli stanno dinanzi. Se aggiungeremo a questi fatti l'altro notevole che il frammento fu montato sopra una tavola ed esposto in Mluseo in modo da mostrare la faccia inferiore anzichè la superiore e che in tal guisa esso si presenta a prima vista sotto un aspetto veramente strano, sarà facile lo spiegarci il perchè tale fossile sia stato si a lungo esposto e sia passato sotto gli occhi di tutti quelli cle visitarono le Collezioni, il piì soventi in fretta, senza arer trovato chi ne desse la giusta interpretazione.

Intraprendendo la rivista dei nostri Cetacei fossili, ho per prima cosa fatto voltare questo pezzo e, quantunque mutilato, non tardai a riconoscere in esso alcune delle principali caratteristiche distinguenti il Plesiocetus (Balacnoptera) Cortesii.

T'schio. - Sulla faccia superiore del teschio noi distinguiamo dapprima l'occipitale ricoprente tutta la volta craniana, depresso sulla linea mediana el un po' rigonfio ai lati, appuntito allo avanti e sporgente ai fianchi in modo da mascherare in parte le fosse temporali, mentre posteriormente esso mostra il foro occipitale, la di cui apertura è tutta quanta visibile dallo insù, fiancheggiato dai condili occipitali anch'essi collocati molto più in su del margine posteriore del cranio e ciò in un grado molto più spinto di quanto abbiamo veduto nello scheletro di Montafia.

Tanto i condili occipitali quanto il margine posteriore craniano sono molto deteriorati; scorgesi però ancora la traccia di profonde insenature esistenti allo indietro fra i condili e gli angoli esterni dell' occipitale. Le zone parietale e frontale non sono pressochẻ visibili sulla linea mediana del cranio, sia per essere ridotte a strettissimi bordi per l'avanzarsi della punta anteriore dell'occipitale fin presso all'oryine delle ossa nasali, sia perchè trattandosi di un esemplare molto adulto e tanto più molto guasto per varie cause, le suture non sono assolutamente visibili che in regioni molto limitate. 
Le ossa nasali, rotte anteriormente, nascevano, come già dissi, a poca distanza allo innanzi dell'estremita anteriore dell'occipitale, presso a poco allo stesso livello colle apofisi posteriori degli intermascellari e dei mascellari. Le ossa temporali e le loro apofisi sono quasi completamente distrutte, non ci possono quindi fornire alcun dato.

A chi guardi di fínco questo frammento di cranio non può mancare di fare impressione l'estrema depressione della sua regione cerebrale. Tale carattere già molto accentuato per se stesso viene ancora esagerato da due fatti, l'uno dei quali inerente al cranio stesso, l'altro dovuto ad estranee cause; essi sono: il primo, l'estremo sviluppo allo infuori della squama occipitale per raggiungere la quale il parietale da ciascuna parte ò pur costretto ad abbandonare nella parte superiore la propria direzione verticale ed a ripiegarsi allo infuori, mascherando in tal modo a chi guaxdi di sopra tutta la parete interna della fossa temporale con una cresta laterale sporgente da tre a quattro centimetri; l'altro è dovuto alla compressione dal basso in alto subita dal cranio durante e prima della fossilizzazione, per cui l'altezza verticale della fossa temporale fu scemata, essendo rientrati l'un dentro l'altro diversi frammenti della sua parete interna e l'occipitale superiore assunse quella posizione cosi superiore e cosi poco posteriore che abbiamo a suo luogo notata.

Molto guasti sono pure $\mathrm{i}$ frontali, dei quali non è discernibile il bordo posteriore del processo orbitale: tanto però possiamo dire che queste erano dirette ben trasversalmente dallo indentro allo infuori, che il canale del nervo ottico correva esso pure affatto trasversalmente e molto presso al margine posteriore dell' apofisi e che infine l'arcata orbitale era ampissima e poco convessa.

Sulla faccia inferiore del cranio sono ben visibili: la regione occipito-sfenoidale e la sfcno-etmoidea, e davanti alla lamina trasverso-etmoidea i due fori d'ingresso agli sfiatatoi; dinanzi a quelli poi una porzione delle ossa palatine. Allo infuori ed allo indietro del cranio due grandi cavità ci indicano le regioni da cui caddern gli apparati uditivi di ciascun lato ed allo infuori di quello di sinistra è ancor visibile porzione della faccia articolare per ricevere il condilo della mandibola. Tutte le parti visibili sono costrutte ed allogate secondo il piano generale che siamo arrezzi ai osservare nella base del cranio delle Balenottere viventi e non offrono altra caratteristica che quella di una estrema riduzione in lunghezza delle regioni corrispondenti alla caritì cerebrale, ciò che del resto è già noto, avendo il Van Beneden fatto rimarcare questo fatto per le Balenottere di Anversa ed avendone già fatta menzione noi stessi a proposito di questa stessa specie ed esemplare.

E d'uopo, prima di lasciare questo pezzo, che io tolga un dubbio che ben potrebbe insorgere in chi, dopo aver letto il lavoro di Borson, leggesse questo mio. Infatti il Borson parla di due forami esistenti sulla facc:a superiore (quella che to tho ora descritta come inferiore) ed internantisi nel cranio. Di questi è ben naturale e constatabile la presenza, erl io li ho pure menzionati piủ in alto quali aperture delle narici inferiori: ma il Borson parla ancora di due altri buchi pure internantisi nella cavità craniana ed esistenti sulla faccia che egli chiama inferiore e che io ho invece collocata superiormente. Tali due fori collocati nella regione posteriore dell'occipitale superiore ed ad un paio di centimetri allo innanzi del gran foro occipitale sono affatto accidentali: essi sono dovuti allo sfondamento locale della parete craniana e non erano

8 A. PORTIS. 
che un solo ed unico foro allargato e corto il quale venne alle due estremita approfondito collo scalpello e che si mostra ora come due cavita distinte. Non hanno per conseguenza niuna ragione per essere tenuti da conto, la loro profondità è affatto artificiale ed è presa nella massa comune rocciosa che riempie tutta la cavità craniana e non hanno un margine definito qualsiasi. A niuno poi potrebbe venire in capo di cercare costi le aperture superiori delli sfiatatoi quando abbiamo trovato molto più anteriormente porzione delle ossa nasali e dopo che abbiamo veduto che le aperture inferiori sono esse ancora collocate assai più innanzi di questi buchi.

Il secondo grande frammento (b) appartenente a questo individuo di Bagnasco mostra, come già dissi, porzione del vomere, dei due mascellari superiori e quasi tutte due le mandibole. Tutte queste ossa sono in un pessimo stato di conservazione, vuoi perchè la parte visibile ne è tutta sdruscita e scrostata, vuoi perchè sono in gran parte ancora ricoperte da roccia sabbioso-calcarea compatta e durissima che è impossibile di levare senza perdere un dopo l'altro i piccoli frammenti in cui è già fin d'ora ridotta tutta la parte ossea sottostante.

Mandibole. - Trascurando adunque di parlare della forma dei mascellari e del vomere, mi limiterò a constatare come le mandibole, vuoi per dimensioni, vuoi per grado di curvatura, si possono affatto considerare come appartenenti alla stessa specie che quelle di Montafia, e che di piủ la parte posteriore di una di esse (la sinistra), che con gran rischio ho in parte liberato dalla roccia, mostra, sia nella forma del condilo che in quella del tallone, e nella posizione della superficie articolare e dello imbocco del canale dentale, perfetta corrispondenza con quanto si osserva nella corrispondente parte della mandibola di Montafia. Ai caratteri osservati alla regione posteriore del cranio aggiungendo ancora quelli che abbiamo trovati sulla mandibola veniamo ad arere una somma tale per la quale possiamo con sicurezza asserire che i due cranii appartennero ad una stessa ed unica specie.

Verlebre e coste. - Le vertebre che abbiamo dinanzi dello scheletro di Bagnasco sommano in tutto ad una quindicina. Fra queste la maggior parte sono dorsali ed alcune poche sono lombari anteriori. Tanto le vertebre quanto i frammenti prossimali di coste che sono conservati presentano gli stessi caratteri e le stesse dimensioni che abbiamo descritti per le corrispondenti parti della Balenottera di Montafia. È quindi inutile il dilungarci di più a proposito di esse.

Nessuna delle ossa delle estremità anteriori e posteriori venne trovata e scavata allorchè si procedette alla estrazione dello scheletro di Bagnasco; o per lo meno nè il Borson ne fece menzione nè io la ritrovai nelle raccolte.

Come conclusione mi giova ripetere che io considero l'individuo di Bagnasco siccome più recchio ma appartenerte alla stessa specie dell'individuo di Montafia, cioè al Plesiocetus (Eulaenoptera) Cortesii. 


\section{N. 11. Plesiocetus (Balaenoptera) Cortesii (DeSn.).}

FiG. 67.68.

LA BaLENotTERA DI CA-LUiga (S. Damano) - N. 19:63.

Il Prof. Gastaldi nella sua lettera al Prof. E. Cornalia in data 20 febbraio 1863, inserta nel $5^{\circ}$ volume delli Atti della Societa italiana di Scienze naturali di Milano, racconta come nel novembre del 1862 sia stato a vedere uno scheletro di Balenottera scoperto presso la stazione di San Damiano (luogo detto Ca-lunga, in circondario d'Asti) nell'argilla azzurrognola pliocenica; come questo scheletro consisteva in sole 35 o 36 vertebre (a partire dalle cervicali) ancora aderenti, a taluna delle quali stava ancora vicina una delle coste; come mancassero affatto il cranio e le natatoie; e come egli avrebbe volontieri fatto acquisto di quel moncone di scheletro, ma che ne fu ritenuto dal prezzo domandato dallo scopritore, prezzo molto superiore a quanto fosse allora in caso di spendere il Gastaldi.

Nell'opuscolo pubblicato nel 1875 dal lodato autore sotto il titolo: Uno schelctro di Balence a Montafia, passando in rassegna i principali resti di Cetacei da lui scoperti prima della Balenottera che occasionò quello scritto, il Gastaldi riparla dello scheletro di Ca-lunga. dicendo come: " A Ca-lunga, nello autunno del 1863 » (leggasi 1862), " un contadino affossando la vigna pose allo scoperto una trentina di vertebre * a cominciare dalle cervicali - le une in serie colle altre: mancava il cranio stato * rotto e stritolato dalle ruote dei carri che passavano lungo una strada profonda" mente incassata nel suolo, sulla sponda della quale affioravano le prime vertebre. "Quel bravo contadino credeva di avere scoperto un tesoro e quando io andai a vedere « quella monca colonna vertebrale me ne chiese parechie centinaia di lire; redendo " poi clie niuno voleva spendere si rilevante somma per possedere quel tesoro, me lo " lasciò per poche diecine di lire quando il sole, la pioggia ed il piede dei curiosi « già l'arevano grandemente danneggiato ».

I residui di questo scheletro, quali vennero raccolti e restaurati dal Gastaldi e quali passarono in seguito al R. Museo di Geologia, non si componevano più clie di 26 vertebre, cioè: le quattro ultime dorsali, dodici lombari e dieci caudali. Esse appartennero ad un individuo ancor giovane ed in cui le epifisi non erano ancora in alcuna delle regioni della colonna saldate ai corpi delle vertebre, mentre le dimensioni di ciascuna vertebra potevano ancor essere al di sotto di quanto raggiungessero nei più grandi individui adulti.

Vertebre dorsali. - Le quattro vertebre dorsali sono di tutte le piu mal conservate. Si mostrano profondamente corrose, una sola fornita di epifisi anteriore mentre le altre ne sono mancanti, tutte sfornite di apofisi trasverse e due anche dell' arco neurale, mentre le altre due ce lo mostrano in un pessimo stato. Possiamo quindi osservare soltanto la relativa brevità dei corpi di vertebra e la loro forma inferiormente arrotondata, l'ampiezza e la poca altezza del canale neurale ed, in quelle che ne sono fornite, la sottigliezza dell'arco neurale. 
Vertebre lombari - Le vertebre lombari sono in assai migliore stato. Con esse possiamo farci un'idea dei caratteri di tutta la regione, le poche che mancano non essendo in serie continua. Noi vediamo i corpi delle vertebre aumentare gradatamente di volume, da $103 \mathrm{~mm}$. di diametro longitudinale (1) per $100 \mathrm{~mm}$. del verticale e per 119 del trasversale (misurati sulla faccia posteriore) che abbiamo trovato sulla prima, a $125 \mathrm{~mm}$. di diametro longitudinale (1) per 119 del verticale e per $137 \mathrm{~mm}$. del trasversale che abbiamo misurato sull'ultima conservata. Vediamo le faccie laterali, sia superiori che inferiori, farsi sempre piu incavate ed una carena longitudinale mostrarsi sulla faccia inferiore; le faccie articolari anteriori e posteriori essere sensibilmente piane ed il foro vertebrale clie nelle vertebre anteriori è triangolare e depresso farsi nell'ultima ancora triangolare ma quasi piu alto che largo. Vediamo i pedicoli dell'arco neurale e tutto l'arco, anche in corrispondenza dell'origine dell'apofisi spinosa, conservarsi sempre sottili e delicati quali già li vedemmo nella regione dorsale di questo indiriduo e di quello di IIontafia. Non possiamo poi dare dimensioni di alcuna delle apofisi spinose, tutte essendo mancanti o rotte presso la loro origine. Iue apofisi articolari son ridotte a due piccoli tubercoli collocati sul davanti dell' arco neurale ed alla sommità di esso, accanto alla origine dell'apofisi spinosa, e mostrano le loro faccette inclinate in dentro ed in aranti.

Le apofisi trasverse poi sono molto sottili di basso in alto, ma invece molto estese sin dall'origine nel senso antero-posteriore, sono collocate man mano pii in giu sui fianchi del corpo fino all'ultima che le presenta a metà altezza; non sono, proporzionalmente, molto sporgenti allo infuori, l'estremità della più estesa non essendo, in linea rettr, distante di più di 14 centimetri dal limite esterno del corpo. Nella loro porzione terminale esse sono espanse un po' più verso l'avanti; lo stesso carattere ho pure riscontrato nelle rertebre dello scheletro di Montafia.

Vertebre canduli (fig. 67,68 ). - Delle vertebre caudali cinque spettano alla regione anteriore ed il loro folume viene ancora aumentando; l'arco neurale e le apofisi spinose ed articolari sono ancora ben sviluppate, il canale neurale è molto più ristretto, le apofisi trasrerse sono un po' meno espanse verso l'esterno, ed in compenso alquanto più verso l'avanti e l'indietro e non sono ancora perforate alla origine; grandissimi sono i tuhercoli posteriori snlla faccia inferiore del corpo delle vertehre, a sostegno delle ossa a $V$, debolissimi gli anteriori.

Le altre cinque caudali sono consecutive; senza essere le ultime di pianta, sono già della seconda metì della serie candale; il canale neurale si va riducendo vieppiu e rengono man mano obliterandosi l'arco neurale e tutte le apofisi; le trasverse, perforat, all'origine in tutte cinque le vertebre, meritano ancora tal nome nelle prime due e non sono piu che tubercoli allungati nelle ultime tre: i processi emapofisarii anteriori hanno raggiunto lo stesso sviluppo dei posteriori e non ne sono piu disgiunti che nolle prime, mentre nelle seguenti si uniscono ciascuno al suo consecutivo formando sulla faccia inferiore della vartebra una carena longitudinale per parte, perforata a metà lunghezza dalla stossa arteria intercostale che forerà consecutivamente

(1) Compreso lo spessore delle due epifisi. 
la base dell apofisi trasversa. Finalmente, avuto riguardo alle dimensioni relative dei corpi di vertebra, il dianetro verticale ha preso il sopravvento sugli altri due, cosicchè nella prima di queste cinque vertebre la lungiezza del corpo raggiunge $129 \mathrm{~mm}$., la sua larghezza $137 \mathrm{~mm}$. e la sua altezza $133 \mathrm{~mm}$., mentre nell' ultima conservata il diametro longitudinale tocca $96 \mathrm{~mm}$., il trasversale $100 \mathrm{ed}$ il verticale $105 \mathrm{~mm}$.

Io ritengo che lo scheletro di Ca-lunga abbia appartenuto alla stessa specie di quello di Montafia, solo che ne sia stato alquanto più giovane. Coll'aiuto di questi due scheletri abbiamo un'idea non solo del cranio ma eziandio dei caratteri della colonna vertebrale e della scapola; coi sussidii che ci dà un quarto individuo, di cui passiamo ora a parlare, conosceremo ancora un'altra parte di non ultima importanza nello scheletro, vale a dire la forma dell'omero.

Finalmente, prima di lasciare definitivamente l'individuo di Ca-lunga occorre notare come il Brandt ne abbia (almeno per disegno) conosciuta l'esistenza; infatti anche di queste vertebre il Gastaldi fece eseguire figure che inviò al Brandt e questi riprodusse i disegni (tav. XXI, fig. 6) nelle sue Untersuchungen, ctc., c ne parlò a pag. 155 e 356 (1) delle medesime, attribuendo le ossa al Cetotherium (Cetotheriophanes?) Cortesii? assieme al cranio della Balaenoptera che noi abbiamo denominnto dal Grastaldi. Da tutto quanto precede si vede come tali due indivilui non possann più stare nella medesima specie; arendo collocato il primo altrove, resta pressochè giusta la denominazione data dal Brandt a questo secondo.

\section{N. 12. Plesiocetus (Ba7nenoptera) Cortesii (Desir.).}

Fic. $69.7 \%$.

\section{LA BALENOTERRA DI CASTELNUOVO CILCEA.}

Da lungo tempo esisterano nella Collezione del $\mathrm{R}$. Museo Mineralogico di T'orino tre pezzi di un Cetaceo fossile, stati trovati nel terreno pliocenico a Castelnuovo Calcea. Due di essi erano stati riconoscinti dal Prof.E. Sismonla come arenti appartenuto ad una Balenottera ed egli li avera fatti disegnare fig. 1 e fig. 2 della Tarola illustrativa dei Cetacei fossili picmontesi e liguri; il terzo, quantunque non sia stato fatto disegnare, era pure da lui stato determinato come un frummento del teschio.

Nessun altro pezzo della stessa località ed individuo venne poscia scoperto; bisogna dunque limitarci per lo studio agli stessi esemplari di cui dispose il Sismonda, cioè :

a) Un frammento di cranio corrispondente alla regione basisfenoidea;

b) La porzione posteriore dellia mandibola destra:

c) L'omero destro.

(1) Avvertasi che il Brandt, dietro i soli disegni speditigli, avanıò di una vertebra nel distinguere le dorsali dalle lombari, cosicchè ne sarebuero rimaste cinque delle prime. L'esame però della vertebra segnata $e$ nella sua figura $\mathrm{mi}$ ha convinto non poter essa certamente venir confusa colle dorsali e dover invece venir tenuta come lombare. Lo stesso ripetasi della vertebra segnata $m$ nella sua figura e che egli considera come ulima fra le lombari presenti (nella spiegazione delle tavole, pag. 356) e che io potei verificare, specialmente colla presenfa dei diso tubercoli pcstero-inferiori di sostegro all'osso a $V$, come appartenente invece alla regione caudale. 
Per cio che riguarda il primo pezzo (a), non ci dilungheremo gran fatto; così isolato ed in cattivo stato quale esso si trova non è possibile, dopo determinata la posizione sua nel teschio, di sottoporlo ad un esame comparativo e di dedurne caratteri differenziali con altre specie. Mi limiterò quindi a dire come esso faccia l'effetto di essere molto ristretto ai fianchi, come del resto si osserva nella corrispondente regione del teschio di Montafia precedentemente descritto.

Mamdibolı (fig. 69,70 ). - Molto piu importante invece è il secondo pezzo (b) che, come già accennai, è la porzione posteriore della mandibola destra.

In essa noi vediamo assai ben conservata la faccia interna, completi il condilo articolare ed il. tallone; piu imperfetti: la faccia esterna, l'imbocco del canale dentale ed il bordo superiore. La faccia interna è quasi piana e molto convessa la esterna, come per le mandibole dello scheletro di Montafia; anche l'imbocco del canale dentale pare siasi comportato nello stesso modo che in quello. Il condilo articolare si mostra molto elevato, ristretto, colla faccia articolare tutta rivolta posteriormente; esso è distinto dallo sporgente tallone per mezzo di un canale collocato sulla faccia interna e destinato al passaggio dei tronchi nerrosi e sanguigni che imboccheranno il canale dentale e che è assai piủ marcato e profondo di quello che nella stessa posizione riscontrammo sulla mandibola del cranio di Montafia.

In complesso i caratteri visibili sono gli stessi e soltanto più marcati di quelli che riscontrammo nella mandibola della Balenottera di Jontafia e ci autorizzano a collocare assieme a quella e nella stessa specic anche gli avanzi della Balenottera di Castelnuovo Calcea.

Cosi stando le cose, noi possiamo arricchire le nostre conoscenze sullo scheletro del Plesiocetus (Balaenoptera) Cortesii ancora dei dati relativi ad un osso di non ultima importanza, vale a dire dellomero, quello trovato a Castelnuovo Calcea essendo molto bello e completo, mentre la Balenottera di Montafia ne era mancante.

Omero (fig. 71, 72). - L'omero di Castelnuovo Calcea è compreso in una lunghezza massima di $275 \mathrm{~mm}$. ed in una larghezza di 159. Il suo condilo è molto grosso, molto rivolto verso la faccia esterna dell'osso e separato dal corpo di quello da un breve ed ampio collo molto ben distinguibile tutto allo intorno ma sovratutto superiormente ed esternamente, dove per esso restano molto ben distinti da una parte il condilo stesso, dell'altra il gran tubercolo. Quest'ultimo è poco sporgente sul bordo inforiore dell'osso ed alquanto di più verso la sua faccia interna.

Il corpo dell'omero, al disotto del condilo e del grande tubercolo, è assai appiattito dallo esterno allo interno, in grado diverso però, a seconda che lo si osserva in prossimità della faccia superiore o del borilo inferiore presso al quale presenta il massimo di appiattimento. In prossimità della faccia articolare per l'avambraccio, esso si appiattisce di più ed, in tal caso, egualmente verso la faccia superiore e verso quella (o bordo) inferiore, e si allarga leggermente. Il piano della faccia articolare pel radio è quasi normale al corpo dell'osso di cui occupa più della metì della larghezza ed è separato per mezzo di una cresta molto sporgente dalla faccia articolare pel cubito, collocata più ad angolo coll'asse dell'omero e che passa verso l'esterno nella seconda faccetta destinata alla porzione olecranica e posta quasi parallelamente all'asse dell'osso.

L'omero di Castelnuovo, cosi slanciato com’è, non presenta guari analogie cogli 
altri ossi dello stesso nome fino ad ora trovati in Italia. L'unico a cui parreble accennare sarebbe quello di San Lorenzo in Collina descritto dal Capellini; pecsato soltanto che quel pezzo sia molto difettoso, cosicche le analogie si riducono alla somiglianza nel grado di compressione della meta distale dell'osso e nella posizione delle faccie articolari per l'avambraccio. Lo stesso omero di Castelnuovo differisce da quello del Museo di Milano (Cortesi 1806) per essere piủ lungo e più compresso di corpo, e per avere il condilo collocato più allo esterno e piủ infuori dal piano normale all'asse dell'osso.

Cosi pure, oltre alle dimensioni di gran lunga maggiori, differisce da quello della Balaenoptera Gastaldii per il corpo dell'osso comparativamente piủ lungo e più compresso, per minor inclinazione delle faccie articolari per l'avambraccio, e per la presenza di una cresta saliente fra di esse.

L'omero a cui più si assomiglia quello di Castelnuovo sia nella forma e posizione delle varie parti, sia nelle dimensioni relative è quello della Balaenoptera borcalina di Anversa descritto dal Van Beneden e da lui figurato (tav. 72, fig. 1, 2), nel quale, a parte le dimensioni assolute, ravriso quasi tutti i caratteri di quello che ho dinanzi.

È nota la grandissima variabilità cui possono andare soggette le varie ossa dello scheletro in diversi individui delle stesse specie di Cetacei. Fondandomi su questo mi azzardo, malgrado il divario che corre fra l'omero di Castelnuovo Calcea e quello dello scheletro di Milano, a ritenerli entrambi come appartenenti alla stessa specie, a ciò principalmente indotto dai caratteri tratti dalla mandibola cui il primo appartenne e che abbiamo superiormente accennati: essi ci insegnano come lo scheletro di Milano, quello di Montafia e quello di Castelnuoro Calcea devono essere compresi in un sola e medesima specie.

Ed ora passiamo, con maggior rapidità, in rivista altri avanzi di minore importanza, sempre provenienti da località piemontesi o liguri.

\section{N. 13. Plesiocetus (Baluenoptera) Cortesii (Dess.).}

Verbebre lombari e couduli. - Riferisco al Plesiocetus Cortesii una serie di 18 vertebre lombari e caudali trovate nell'Astigiana e da lungo tempo conservate nelle Collezioni del R. Iluseo di Mineralogia e Geologia.

Spettano probabilmente all uno stesso individuo; non abbiamo però dati certi e nessuna indicazione precisa che ciò sia. È certo però che l'individuo (o gli individui) cui queste vertebre appartemero era affatto adulto quando mori; infatti esse ne mostrano tutti i caratteri.

Le dimensioni sono assai più forti che non per le vertebre di Ca-lunga, queste essendo giovani, quelle, come osservai, adulte. Del resto esso sono ridotte ai soli corpi, quindi, allo infuori delle dimensioni e della forma loro, niun altro carattere atto alla determinazione specifica ci viene da loro fornita. 


\section{N. 11. Plesiocetus (Balacnoptera) Cortesii (Dessi.).}

Mandibola, vertebre e cosle. - Similmente riferisco alla stessa specie molti avanzi di uno scheletro che da lungo tempo fu scarato nell'Astigiana dalle sabbie gialle e trasportato nei magazzini del IIuseo Geologico.

Sono in tutto nove o dieci grossi massi di sabbia, in parte cementata ed in parte no, nei quali riposano e sono ancora in parte da essa ricoperti:

a) Una grossa porzione di mandibola lunga quuasi due metri;

b) Una dozzina di vertebre dorsali, lombari, cutudali ;

c) Iolte coste e frammenti di coste.

Tutte le ossa concordano, per quanto è dato di velere, con quelle del Plesiocetus Cortesii descritto ai numeri precedenti ed appartennero ad un grosso individuo adulto.

\section{N. 15. Plesiocetus (Bulaenoptera) Cortesii (Desy.).}

Verlebre rachiliche. - Nei magazzini del ILuseo di Geologia esiste una serie di 14015 vertebre ridotte ai soli corpi, che appartennero ad un individuo malato. Esse sono tutte profondamente corrose e deformi e spettavano alle regioni dorsale e lombare. Lindividuo di cui fecero parte era probabilmente della specie Plesiocetus Cortesii. Hanno però dimensioni minori, cosa che si spiega facilmente collo stato altamente rachitico dell'animale di cui fecero parte.

\section{N. 16. Plesiocetus (Bulucnopteru) Cortesii (Desxr.).}

Verlebre lombari c cundali. - l're vertebre, di cui due lombari ed una caudale, state raccolte a Bagnasco molti anni addietro e forse prima che nella stessa località si trovassero gli avanzi dello scheletro di cui al N. 10. È probabile che non solo appartengano alla stessa specie di quello, ma fors’anco allo stesso individuo.

\section{N. 17. Plesiocetus (Bulacnoptera) Cortesii (DEsur) -- 19264.}

Asse. - Due vertebre raccolte nel Pliocene di IIontafia (regione Vatalon) dal Prof. Gastaldi. L'una è una vertebra asse ridotta al solo corpo. T'ale vertebra è asimmetrica, l'apofisi trasversa inferiore sinistra essendo molto meno sviluppata della destra; cosi pure il pedicolo sinistro per l'arco neurale molto meno allargato del destro ed il processo odontoide essendo molto spostato verso sinistra. La faccia articolare posteriore è molto larga e bassa.

Verlebri caudule. - L'altra vertebra è una caudale ridotta al solo corpo e questo molto maltrattato. In complesso queste due vertebre paiono aver appartenuto 
alla stessa specie dell'altro individuo di Inontafia del quale hanno pure lo stesso modo di petrificazione, per riempimento cioè dellı carità e porosità delle ossa con calcare. Per questo le ossa stesse rengono ad acquistare una molto maggior densità che non le altre della stessa specie provenienti da altre località dell'Astigiana.

\section{N. 18. Plesiocetus (Balaenoptera) Cortesii (DESM.).}

Vertehra cervicule. - Una vertebra cervicale raccolta dal Gastaldi in località non determinata dell'Astigiana. L̀ probabilmente la $6^{\text {a }}$ cervicale. L̀ molto grande, appartenne ad un individuo affatto adulto e spetta probabilmente al Plesiocetus Cortesii.

\section{N. 19. Plesiocetus (Balaenoptera) Cortesii (Des.r.).}

Tertebra dorsule. - Una vertebra dorsale mediana, incompleta, raccolta dal Gastaldi in località non precisata dell'Astigiana. Presenta, per quanto è visibile, i caratteri che distinguono la Balenottera di Montafia.

\section{N. 20. Plesiocetus (Balaenoptera) Cortesii (Desur.) -9510.}

Vertelie dorsuli. - Sono cinque corpi di vertebre trovate dal Gastaldi nell'Astigiana, ma senza più precisa indicazione.

Di queste, due sono dorsali, molto grandi, adulte e presentanti i caratteri di rachitismo di cui dicemmo al N. 15. Sono però deformate in molto minor grado, e mentre da un lato si possono ancora comparare con quelle degli individui sani della Bulaenoptera Cortesii e riferire alla stessa specie, dall'altra ci dànno la chiave per ulteriori modificazioni patologichè e per riferire quindi sempre a quella specie anche le vertebre del succitato $N .15$

Jeridbre camduli. - Le altre tre vertebre appartengono alla regione caudale; di queste una alla porzione anteriore, anzi probabilmente la prima caudale, presenta pure alcuni caratteri di rachitismo; l'altra alla posteriore, si mostra allo stato normale.

La terza poi, quantunque sia forse stata trovata assieme, non spetta sicuramente allo stesso individuo delle quattro precelenti. is intermedia, per posizione in serie, alle due caudali preaccennate, ma è molto più piccola della seconda ed in proporzione piủ lunga. Sarei per riferirla anzichè alla $B$. Cortesii, piuttosto alla $B$. (r̆ $Q-$ staldii, di cui presenta molti caratteri.

\section{N. 21. Plesiocetus (Balachoptera) Cortesii (Desu.).}

Verrebre caudali. - Nella regione Varason, a Mongiaglietto presso Cortandone, vennero trovate dal Gastaldi due vertebre caudali (posteriori) ed un frammento di costa. Per dimensioni, proporzioni e forma paiono riferibili al Plesiocetus Cortesii.

9 A. PORTIS. 


\section{N. 22. Plesiocetus (Balaenoptera) Cortesii (Desa.).}

Vertebra lombure. - Una rertebra (corpo) lombare raccolta dal Prof. Gastaldi nell'Astigiana, rotta in due parti e molto maltrattata. Forse riferibile al $P$. Cortesii.

\section{N. 23. Plesiocetus (Balacnoptera) Cortesii (Desn.).}

Verlebre combluli. - Due vertebre caudali, una anteriore, una posteriore, spettanti ad un individuo giovane e riferibili alla stessa specie. Fanno da lungo tempo parte delle Collezioni del R. Mfuseo e provengono dalle sabbie gialle dell'Astigiana.

\section{N. 24. Plesiocetus (Bulanoptera) Cortesii (Desm.).}

Verlebr'l cumlule. - Una vertebra caudale anteriore di un individuo adulto della stessa specie fu raccolto in Talle Andona assieme ad alcune altre. Mi fu confidata dal Dott. Chiosso, Professore di Storia naturale al $R$. Liceo di Asti e fa parte ¿lel Gabinetto di quel IR. Istituto.

\section{N. 2:. Plesiocetus (Bulucnoptora) Cortesii (DESAr.).}

levelura coudule. - Una rertebra candale media, assai bene conservata, trorata nelle vicinanze di Montiglio e regalata al R. AInseo dal signor Mtarchese di Cocconito. Quantunque, relativamente al proprio posto in serie, un po' corta, pare anch'essa riferibile al Plesiocetus Cortesii. Appartenne ad un individuo adulto e, relativamente alla specie, di assai grandi dimensioni.

\section{N. 2(i. Plesiocetus (Bul(cenopteru) Cortesii (DESנr.).}

Verledire cunduli. - Dne vertebre candali (regione media) di un individuo giovane; mancano delle due epifisi. Paiono riferibili alla $B$. Cortesii, quantunque relativamente un po' corte. Sono di assai piccole dimensioni; l'una di esse porta aderente, per mezzo della sabbia, una piccola ed insignificante scheggia di costa. Fanno da lungo tempo parte delle Collezioni di questo R. IIuseo e provengono dall'Astigiana senza più precisa indicazione di localitì.

\section{N. 27. Plesiocetus (Batuenoptera) Cortesii (Desn.).}

Verlebra cuudule. - Una rertebra caudale dell'Astigiana, raccolta dal Professore Gastaldi. Appartenne ad un grosso ed adulto individuo, è assai ben conservata; è la penultima o terz'ultima della colonna vertebrale ed appartenne probabilmente alla specie Plesiocetus Cortesii, se non ad una specie molto più grande. Ne riparleremo a proposito di quella del N. 36. 


\section{N. 28. Plesiocetus (Balucnoptcra) Cortesii (Dess.)?}

FIG. 73.

Ossa " I. - Due ossa a $V$ raccolte nell'Astigiana dal Prof. Gastaldi, ignoro se assieme od in due diverse località. Entrambe sono leggerissime in proporzione delle altre ossa di Balenottera, entrambe hanno ben conservate le porzioni articolari superiori, entrambe son rotte nella porzione inferiore o laminare, in entrambe lo due metà dell'osso sono completamente unite e fuse. Questo carattere e lo sviluppo delle faccie articolari dimostrano aver esse appartenuto ad un individuo gia affatto adulto. Di esse l'uno doreva essere collocato di almeno 5 o 6 posti allo avanti dell'altro. L'uno, l'anteriore, misura dallo avanti allo indietro del suo corpo laminare mediano un 9 centimetri, l'altro un 5 centimetri. Ho fatto disegrare (fig. 73) il posteriore perchè piủ completo. Credo che abbiano appartenuto entrambe alla specie $B a-$ Tacnopterc Cortesii.

\section{N. 29. Plesiocetus (Balamoptera) Cortesii (Desur.)?}

Costu. - Un moncone prossimale di una delle coste collocate piủ allo indietro di una Balenottera, che fu probabilmente la $B$. Cortesii, fu trovato nell'Astigiana dal Prof. Gastaldi.

\section{‥ :30. Plesiocetus (Butrenopteru) Cortesii (Desm.)?}

Cranio (rottami). - Due frammenti insignificanti della parte posteriore del cranio, probabilmente della regione sfenoidea, trovati dal Prof. Gastaldi nell'Astigiana.

\section{N. B. Plesiocetus (Balcenoptera) Cortesii (Desar.).}

FIG. 77.

C'asse limpanichc. - Nell'Astigiana, e probabilmente a Dusino vennero raccolte. negli anni addietro, due casse uditive che il Prof. E. Sismouda riconobbe come appartenenti ad una Balenottera e clie egli intendera di illustrare arendone gia fatti eseguire $\mathrm{i}$ disegni (fig. 15 e 16 della tarola già varie rolte citata). Esse sono la destra e la sinistra, probabilmente, dello stesso individuo. Quantunque le dimensioni loro sieno alquanto minori, tuttaria la forma loro concorda esattamente con quella della cassa auditiva che descrivemmo al N. 9. trattando dello scheletro di Montafia. Le riferiamo per conseguenza alla stessa specie di quella. 


\section{N. 32. Plesiocetus (Bulaenoptera) Cortesii (Desur)?}

Fig. 78.

Roccia. - Il Prof. Gastaldi raccolse ancora nell'Astigiana un piccolo osso che riconobbi per il complesso delle ossa periotiche o roccia (rocher) destra di una Balenottera. È fin qui l'unico che si sia trovato in Piemonte, e manca della grossa apofisi che lo teneva unito al temporale. Per la forma sua e per le sue dimensioni ricorda molto quello della Balaenoptera boreatina V. BEx. di Anversa rappresentata dal Van Beneden a tar. 78, fig. 9-10, nel volume $3^{\circ}$ della sua Descrizione delle ossa fossili del bucino di Anersa. Anzichè una descrizione difficile a farsi ed a comprendersi, preferii dare una figura di questo prezioso pezzo, facendolo riprodurre (fig. 78 del presente lavoro).

Non ho dati per ammettere con sicurezza che questa roccia abbia veramente appartenuto al Plesiocetus Cortesii, ma farò notare che tale specie è la più comune in Piemonte, la maggioranza degli avanzi fin qui trovati nell'Astigiana appartenendo ad essa. Le piccole dimensioni dell'osso che abbiamo dinanzi stanno in proporzione abbastanza giusta con quelle delle altre ossa dello scheletro del Plesiocetus Cortcsii, sovratutto poi colle dimensioni delle piccole casse timpaniche che sappiamo aver desso possedute. Ammettiamo quindi fino a prova contraria che la roccia in questione sia veramente una roccia destra della specie che siamo renuti trattando fin qui.

\section{N. 33. Plesiocetus (Balaenoptera) Cortesii (Desur).}

Vandibola. - Un frammento posteriore di mandibola sinistra in cui è visibile parte del condilo, il tallone, e l'imbocco del canale dentale. Quantunque il condilo sia alquanto più sviluppato trasversalmente, il tallone un po' più sporgente allo indietro e l'imbocco del canale dentale alquanto piu guardante allo insủ di quanto redemmo negli altri esemplari di Plesiocetus Cortesii, che venimmo fin qui enumerando, tuttavia queste piccole differenze possono venir ritenute come individuali, l'aspetto e la forma generale del frammento invitandoci a considerarlo sempre come della stessa specie,

Il frammento, appartenente ad un individuo adulto, venne raccolto dal Professore Gastaldi nelle sablie gialle dell'Astigiana.

\section{N. 34. Plesiocetus (Bafnenoptera) Cortesii (DEsu.).}

Omero. - Nei magazzini del Museo di Geologia ho trovato un magnifico omero sinistro di Balenottera, proveniente dall'Astigiana. Paragonatolo con quello di Castelnuovo Calcea descritto al N. 12, trovai che si accordarano assieme molto bene, sia per riguardo alle dimensioni generali, sia per la posizione e direzione del condilo relativamente al corpo dell'osso, sia per la presenza di un piccolo collo, sia per lo sviluppo del grande tubercolo, sia infine per la compressione della testa inferiore 
dell'omero e per la posizione e sviluppo relativo delle faccie articolari per l'avambraccio. Una sola differenza si nota, ed è una maggiore compressione ed ur inaggiore allargamento del corpo dell'osso nella sua regione media, sensibili in questo omero sinistro. Dero peró notare che questo pezzo $\dot{e}$, sarei per dire, petrificato, cioè é tutto imberuto allo iuterno di calcare, quindi pesantissimo, mentre di quello di Castelnuovo Calcea non è che conservata la porzione minerale senza infiltrazione di nuovo materiale. is quinli naturale lo ammettere che la pressione a cui sottostette quest'osso, mentre era tutto inzuppato dall' acqua che lo infiltrò pui di calcare, In abbia alquanto appiattito ed allargato, ma che la forma originale fossere afiatto la simmetrica per quello a cui lo abliamo paragonato.

\section{N. 33̈. Plesiocetus (Balaenoptera) Cortesii (Dessr.)?}

FIG. $74,73$.

Cubito. - Sempre dal Profo Gastaldi e sempre nelle sabbie gialle dell'Astigiana. renne trovata la meta prossimale di un cubito sinistro arente appartenutn al un indiviluo ancora giorane di Balenottera, ancor giovane, poichs le epifisi non erano ancora saldate alla diafisi ed andarono perdute. Contuttocio le sue dimensioni sono già assai superiori a quelle del cubito sinistro della Binlucmoptera Grastuldii a cui l'ho comparato. Differisce poi dal medesimo, oltreche per le dimensioni, per arere aruto il corpo dell'osso piu appiattito ed allargato, e par avere il processo olecranico piu rirolto in basso e quasi ad angolo retto col corpo dell'osso. l'er quest'ultima particolarità differisce pure dal cubito dello scheletro del Juseo di Bologna e da quello di tutte lo Balenottere di Anrersa, mentre molto si accosta a ruello del Huseo di Milann.

Benclie sia stato trorato isolato, cred', che quest'ns:n abhia apparte:uto ad un giovane individuo di P'lesincetus Cortesii. Gli scheletri a parti di scholetri piemontesi fin qui enumerati ne mancarono ognora; ho per conseguenza creduto bene di dare il disegno di quest'unico esemplare, come del culjito della specie in ruestione, persuaso che il tempo, conducends a sonperte di nuovi aranzi, darà rarione a questo min modn di sedere.

drun rue con una grande quantità di avanzi appartenenti alneno a 27 scheletri differenti, quasi tutti raccolti in Piemonte e particolarmente nell Astigiana, roi siamo renuti arl arere dinanzi quasi tutto lo scheletro della Jinlamoptern ('ortesii e ci abljiano) potuto fare un'idea della forma delle rarie ossa, dei lorn rapporti con quelle di altre Balenottere riventi of fossili e delle dinensioni dello intero animale, il quale iloreva misurare in lunghezza almenn 8 metri e rnezzo; compreso il cranio. Siamn felici che il Van Beneden abbia prima di noi accennato all'eruaglianza specifia tra la Halenottera I'iacentina (del Museo di Milano) e quelle dell'Astigiana. Noi althiano ammessa solo in parte la sua teoria, in quanto doremmo distinguere le Balenottere dell'Astigiana in tre specie almeno, delle quali due rennero di già descritte, nentre accenneremo tosto alla terza. 
Fra queste tre specie, la più comune e più copinsa in individui è quella appunto che si identifica con quella stabilita sullo scheletro del Museo di Milano. Il Van-Beneden aggiunge poi che la Balaenoptera Capellinii del IIuseo di Bologna e del Inseo di Parma, e la Balacnoptera Cortesii del Museo di Parma appartengono ancora sempre alla stessa specie dello scheletro del Museo di Milano e per conseguenza di quelli del IIuseo di Torino. Ammettendo per ora, senza discutere, una tale determinazione, noi veniamo a conchiudere che la regione in cui visse, durante l'epoca pliocenica, la Balacnoptera Cortesii abbracciava per lo meno la Liguria settentrionale, tutta la vallata del $\mathrm{Po}$ ed il versante orientale dell'Appennino fino al di sotto di Bologna.

Vediamo ora altri aranzi di Balenottere del nostro paese, che non si possono identificare nè colla $B$. Girstuldii, nè colla $B$. Cortesii.

\section{N. 3(i. Balaenoptera n. sp. A.}

Dalle sabbie gialle dell' Astigiana fu raccolta molto tempo addietro e conservata nel R. IIuseo di Mineralogia e Geologia ma grossa vertebra di Balenottera. Quantunque il sno modo di fossilizzazione e le fresche traccie di rottura sorra essa esistenti ci dimostrino che essa doveva esistere intiera in seno al terreno, tuttavia, per essere stata trovata forse a caso e da individui che non si curarono di raccogliere i minori frammenti, essa si trova ora molto incompleta, essendo ridotta al solo corpo, e questo ancora ben difettoso e mancante.

La vertebra appartenne alla regione lombare posteriore; essa misura dallo avanti allo indietro $233 \mathrm{~mm}$. e, sulla faccia posteriore, verticalmente $190 \mathrm{~mm}$, trasversalmente $220 \mathrm{~mm}$. Le faccie articolari anteriore e posteriore sono sensibilmente piane: il canale neurale cra già proporzionalmente molto ristretto ed i pedicoli dell' aren dimostrano come esso fosse molto allungato ed a pareti sottili, mentre fortissime e molto sviluppate doverano essere le apofisi trasterse.

Certameute non si può molto conchiudere da questo pezzo, quanto ad analogie con tale o con tal'altra specic rirente o fossile, ed io mi limiterò a far notare la depressione naturale di questo corpo di vertebra in proporzione delle altre due dimensioni, cosa del resto evidente dal solo esame delle tre cifre che vengo di dare, e le dimensioni sue che ci indicano arer esso appartenuto ad una specie molto più grande di quella che ci ha da ultimo tenuti si a lungo occupati, e come essa non doveva stare di molto indietro a quella che ancora oggidi visita ad intervalli il nostro litorale, vale a dire la Balaenoptera muscutus.

Nel bacino di Anversa rinviensi fossile una Balenottera cle anch'essa trovasi in questo caso e che ricerette il nome di Balaenoptera musculoïdes; le dimensioni sue sono alquanto minori di quelle che ci sarebbero indicate dal pezzo piemontese. La forma e le dimensioni relative potrebbero invece andare per le due sensibilmente d'accordo. Potrassi forse col tempo ammettere che la Balarnoptera musculoüdes sia pure stata, durante il periodo pliocenico, a visitare la valle del Po? 
N. 37. Balaenoptera n. sp. $A$.

Una seconda grossissima vertebra, appartenente ad un altro individuo, venne pure negli ami addietro trovata nell'Astigiana; anch'essa è una vertebra lombare, le dimensioni sue sono però assai minori di quelle del pezzo precedente, nisurando dallo avanti allo indietro $\mathrm{mm} .212 \mathrm{e}$, sulla faccia posteriore, verticalmente $173 \mathrm{~mm}$. e trasversalmente $210 \mathrm{~mm}$. Essa presenta del resto la stessa forma e gli stessi caratteri della precedente; sovratutto $\dot{e}$ in essa ben visibile e noterole il grande predoninio del diametro trasversale sul verticale.

Essa si accorderebbe, sia per la forma sua, che, a un di presso, per le dimensioni assolute e relative, colle rertebre della stessa regione nella Balaenoptera musculoürtes di Anrersa e ci rende probabile l'ipotesi enunciata a proposito del pezzo precedente, che cioè tale specie abbia anche visitato il nostro bacino pliocenico. La differenza poi di volume fra le due vertebre, portante naturalmente a corrispondente differenza fra $\mathrm{i}$ due individui che le possedettero, non è tale da impedirci di ammetterli entrambi in una stessa specie. Il Tan Beneden ci ha dimostrato, pei Cetacei di Anrersa, ben più grandi differenze di statura fra individui adulti della stessa specie; quindi se noi ammettiamo che il pezzo N. 37 abbia appartenuto ad un individuo di statura media per la specie, ammetteremo che il pezzo N. 36 proveriga da un indiriduo gigantesco per la specic, o vicerersa se la statura media fu rappresentata da questo secondo, noi possiamo figurarci che l'individuo di cui fece parte la vertebra distinta dal N. 37 fosse nano relatiramente alla specie.

Parlando al N. 27 di una penultima rertebra caudale trovata nell'Astigiana dal Prof. Gastaldi, feci osservare come le dimensioni di quella accennassero ad un individuo di gran lunga superiore al taglio ordinario per il Plesiocetus Cortesii. Ed infatti essa ¿̀ ancora più grande della vertebra caudale rentesima della Bulacnoptera musculoülos di Anversa, raprresentata dal Tim Beneden nella più volte citata $3^{\mathrm{a}}$ parte dei suoi Osscments fossites thu bussin d'Amers (tar. 58, fig. 5 e tav. 60, fig. 4), della quale è pure piủ corta. Potreblue benissimo darsi il caso clıe ella non fosse punto riferibile al Plesiocéus Cortesii nella cui lista l'abbiamo collocata e che invece appartenesse alla stessa specie delle due vertebre menzionate ai numeri 36 e 37 del presente lavoro e, con esse, rappresentasse forse la Balacnoptera musculoüles V. Ben. nei nostri terreni terziarii.

\section{N. BN. Balaenoptera n. sp. B.}

Fin. 81.83 .

Láasst limpanicu. - Il Prof. Perez trovò molti anni or sono nelle argille plioceniche delle fornaci di Sarona mna cassa auditiva incompleta di Cetaceo, che volle regalare a questo $R$. Museo. Essa appartiene al lato destro e misura $80 \mathrm{~mm}$. di lunghezza (1) e, nel terzo posteriore dove il diametro trasversale è massimo, $42 \mathrm{~mm}$.

(1) La lunghezza data è un poco inferiore alla reale mancando anteriormente di porzinne del bordo superiore. 
di larghezza. Quantunque quest'osso non sia affatto completo, lascia però distinguere caratteri tali da indurci ad ammettere nel Pliocene ligure una specie di Balenottera ben diversa dalle due che conosciamo gia sufficientemente bene in Piemonte ed anche un poco nella stessa Liguria.

L'intima struttura dell'osso, cosi perfettamente omogenea e sarei per dire pietrosa, che distingue le casse timpaniche dei Histiceti e che le fa talora confondere con ciottoli selciosi, non è ancora cosi ben definita nella cassa raccolta dal P’rof. Perez, dove, invece, nei punti di rottura, troviamo una struttura leggermente porosa e quasi fibrosa, che chiaramente ci indica come l'osso in questione abbia appartenuto ad un individuo ancora giovane.

Se dopo ciò noi facciamo attenzione ai caratteri tratti dalla forma dell'osso, noi vediamo come esso sia molto allungato ed elevato, mentre è pochissimo rigontio di fianco, anzi, e sovratutto nella porzione anteriore, è compresso dallo interno allo esterno e come l'apertura abbia dovuto esser molto bassa ed allungata, mentre il bordo inferiore dell'intiera cassa si mostra debolissimamente appiattito.

Noi sappiamo che un carattere costante il quale serve a distinguere a primo colpo d'occhio le casse timpaniche delle Balene da quelle delle Balenottere è appunto quello della compressione laterale e dell'appiattimento della faccia inferiore, per cui noi riferiamo alle Balene casse auditive compresse dallo esterno allo interno, non appiattite inferiormente ed anzi marcate sul borlo inferiore da una carena più o mono saliente per cui vengono ad assumere l'aspetto di un fondo vuoto di portamonete (Brandt, Van Beneden), mentre riferiamo alle Balenottere delle casse rigonfie, corte, e col margine inferiore (che qui diventa una faccia) appiattito ed esteso.

Noi sappiamo però che tali differenze sono meno sentite nella giovane età, quando la cassa dei Balenidi è ancor pì̀ rigonfia e meno carenata in basso; quindi allorchè noi troviamo sopra la cassa anditiva di Savona i caratteri che vengo di menzionare restiamo in forse sul genere al quale dobbiamo ascrivere l'animale che la possedette.

Egli è solo partendo dallo esame del grado di divaricazione della parete interna dal massiccio che forma la parete esterna e quindi dal grado di apertura del fondo della cavità della cassa che io mi sono deciso a collocare il Cetaceo di Sarona, possessore di una tal cassa, nel genere Balaenoptera. Mentre però mi sono deciso ad una tale classificazione, non posso a meno di insistere sugli indicati caratteri di alta importanza sistematica, i quali ci obbligano ad ammettere che tale Cetaceo, pur essendo Balenottera, dovera possedere grandissime affiniti colle vere Balene e costituire cosi, fra $\mathrm{i}$ due tipi, un ponte di passaggio analogo a quello che, per altre particolarità, vien costituito dai generi Balaemula e Balaenotus.

Come per altri casi precedenti e che già incontrammo nel corso di questo mio lavoro, dobbiamo conchiudere con augurarci che anche per questo abbia a trovarsi prossimamente lo scheletro di un Cetaceo che, distinto dai caratteri delle ossa timpaniche che ci si presentano in quello che abbiamo dinnanzi, possa essere considerato come il tipo di questa nuova specie e nel quale possiamo studiare i caratteri scheletrici ed accertarci che le deduzioni tirate da un solo ossicino non erano lontane dalla verità ed incontravano conferma ed importanza di caratteri nella forma e nei rapporti delle altre ossa. 


\section{N. 39. Balaenoptera n. sp. C.}

Fif. $79-80$.

Verlebra caulule. - Il sig. Prof. A. Issel mi ha gentilmente comunicata una vertebra di Cetaceo che si conserva nel Museo di Mineralogia e Geologia della R. Universitì di Genova e che fu trovata nelle argille plioceniche di Savona.

Tale vertebra appartenne ad un individuo ancor giovane, poichè le due epifisi erano ancora staccate dal corpo della vertebra ed andarono perdute, e spetta alla regione caudale. Il corpo della vertebra misura, cosi senza epifisi, $79 \mathrm{~mm}$. dallo avanti allo indietro e, misurando sulla faccia posteriore, $126 \mathrm{~mm}$. di alto in basso e $120 \mathrm{~mm}$. trasrersalmente. L'arco neurale già molto ridotto, con apofisi articolari rigonfie, tubercoliformi, e molto piủ grosse dell' apofisi spinosa, è a pareti molto robuste e circonda un canale vertebrale già ridotto a sezione circolare di un centimetro di diametro. Cosi pure sono già molto ridotte le apofisi trasverse che, intiere, non doverano sporgere piu di due centimetri allo infuori del corpo della vertebra e sono perforate alla base dal solito canale verticale arterioso. Sviluppatissimi ancora sono invece i tubercoli d'attacco per le ossi a $V$ e sopratutto i posteriori grossi e triangolari.

Questa vertebra, indubbiamente di Balenottera, differisce però da quelle delle altre specie piemontesi fin qui descritte per la sua forma e specialmente per il carattere, che risulta dalle dimensioni date, della sproporzione cioè fra la sua lunghezza e la sua altezza e larghezza. Per questo, mentre da un lato ricorda le Balenottere, si accosta dall'altro ancora ai generi Jegattera e Balena e ci indica come l'animale di cui essa fece parte doveva appartenere ad un tipo o ad un genere intermedio fra i precitati.

Noi arrivammo già alla stessa conclusione pel numero precedente, partendo dallo esame di una cassa timpanica proveniente pure dalle argille di Savona: noi la troviamo ora confermata dal presente pezzo. Può darsi forse che questi due pezzi abbiano appartenuto-entrambi allo stesso sottogenere, fors'anco alla stessa specie (possiamo però affermare che non furono trovati nello stesso giacimento e che non appartengono allo stesso individuo). Per ora mi astengo dal pronunciarmi in modo definitivo e pur descrivendo a parte $\mathrm{i}$ due pezzi come appartenenti a due specie diverse, mi limito a far constatare come in Liguria e nel circondario di Savona si sieno trovate in giacimenti pliocenici, oltre a vere Balenottere, anche una o più specie di Cetacei che accennano a non lasciarsi collocare nè assolutamente nel genere Balaena, nè definitivamente nel genere Balacnoptera, e delle quali non si può nemmeno dire con sicurezza se possano venire ascritte ai generi intermedii già stati trovati nei terreni pliocenici di altre località d'Italia e d'Europa. 


\section{N. 40. Balaenoptera n. sp. D.}

\section{LA BALENOTTERA MIOCENICA DI MONTOLINO.}

Terlebre e coste. - Il Prof. Gastaldi nei suoi Cenni sui Vertebrati fossili del Piemonte ricorda come nel 1855 il sig. Giovani Volpato, Prof. di disegno, trovasse in un suo fondo posto sulle alture di Montolino in territorio di Pino Torinese una porzione di scheletro di Cetaceo che era così il primo Mammifero fossile scoperto sulla nostra collina. Tale prezioso pezzo passò al nostro Museo Geologico assieme agli altri tesori paleontologici radunati dal Prof. Gastaldi e, se non pel suo stato di conservazione, certo per la sua rarità merita clıe ne facciamo pure menzione in questo lavoro.

Le parti conservate consistono in 12 rertebre ed alcune coste distese, le prime in serie sopra un masso di arenaria, e di alcuni frammenti di coste sorra due minori frammenti della stessa roccia.

Delle vertebre, le prime cinque spettano alla regione dorsale posteriore, le altre alla regione lombare anteriore; crescono rapidamente di volume, misurando la prima $40 \mathrm{~mm}$. di lunghezza e la dodicesima $92 \mathrm{~mm}$.; sono tutte molto mutilate e mancanti, alcune soltanto mostrano ancora qualche parte dell'arco neurale e dei processi spinosi i quali sono sviluppatissimi in proporzione del corpo delle vertebre ed inclinati allo indietro, ciỏ che del resto è secondo la generalità delle Balenottere.

Pare che l'animale da cui queste vertelore provengono fosse giâ adulto ed arguisco cio dal fatto che in alcuni punti di rottura fresca credo di aver osservata la linéa di demarcazione fra $\mathrm{i}$ corpi di vertebra e le rispettive epifisi e che queste ultime fossero già completamente saldate ai corpi stessi.

Ciò stando ed essendo pure abbastanza convinto che l'animale di Montolino doresse essere una Balenottera, oserei dire che esso appartenne ad una specie inferiore per dimensioni alla Balacnoptera Gastaldii, precedentemente descritta, e distinta da essa per un rapido accrescimento di lunghezza dei corpi delle vertebre successive alle cervicali, cosicchè essi nella regione lombare posteriore e nella caudale anteriore doverano venire a raggiungere una lunghezza eguale, se non superiore, alle corrispondenti parti della Balacnoptera Gastuddii, mentre ne erano inferiori per le altre dimensioni.

Ad altri rapporti e confronti non è qui il caso di pensare, troppo cattivo essendo lo stato di conservazione dei pezri visibili. Limitiamoci per conseguenza a constatare come anche nel Miocene medio della nostra Collina si sieno trovati gli avanzi di Misticeti di piccole dimensioni, i quali paiono riferibili al genere Balacnoptera.

\section{N. 41. Balaenoptera? sp.}

Anche nei terreni miocenici di Serravalle Scrivia vennero riscontrati avanzi di Cetacei. I due frammenti che finora se ne conoscono appartengono al Museo di Genova e mi furono gentilmente comunicati dal signor Prof. A. Issel. Essi sono ancora troppo maltrattati e troppo piccoli perchè si possa determinare a qual parte spettino 
dello scheletro. Soltanto la struttura dell'osso ci inclica che con tutta probabilità abbiamo a che fare con un Misticeto. Mi limito quindi alla sola constatazione del fatto ed a far notare come questi avanzi provengano pure da un conglomerato friabile ed a grossa grana, nel quale, molto probabilmente, non si depositarono clie parti isolate delle scheletro (1).

\section{PARTE SECONDA}

\section{GETODONTI}

\section{FISETERIDI}

\section{GENERE PRIS COPH Y E TER PORTIS.}

\section{N. 42. Priscophyseter typus Portis.}

Fig. $8 i-80$.

Vertebre cerviculi. - Il Museo di Torino possedera, già da tempo, un frammento osseo ancora indeterminato e senza alcuna esatta indicazione di provenienza. L'aspetto dell'osso lo indica chiaramente come arente appartenuto a Cetacei e la sabbia indurita che lo arvolge ci dimostra abbastanza che esso abbia doruto trovarsi in qualcuna delle località plioceniche dell'Astigiana.

Ripulito in parte il frammento, non tardai ad accorgermi aver dinanzi un pezzo di sinostosi prodotta dalla fusione dei corpi di quattro vertebre consecutive. La maggiore lunghezza del frammento tocca i 177 millimetri, la maggior larghezza $122 \mathrm{~mm}$, la sua altezza $104 \mathrm{~mm}$.

La superficie inferiore, continua colle laterali, si presenta semi-ellittica; essa è perforata da numerosi fori mutritizi che s'internano nell'osso con direzione, or dallo

(1) $\aleph^{\circ} 4$ ! bis. Balaenoptern! Da gentili comunicazioni avute rilevo che nell'anno 1883 il proprietario Negro Giuseppe; di un fondo sito a monte della Villa Giubilino in borgata Rosero (borgata del comune di Pecetto), sul ver'sante meridionale della collina di Torino, nello scavare una cantina che si addentra per oltre 35 metri nel colle trovò una quantità di ossa fossili che, vista la natura del giacimento e degli organismi fossili che vi si trovano, possono aver appartenuto o ad un Sirenio o ad un Cetaceo. Sventuratamente queste ossa vennero disperse e distrutte prima che persona intelligente ne potesse aver contezza e le potesse vedere. E quindi assolutamente impossibile il poterne trarre un insegnamento qualunque.

$\mathrm{N}^{\circ} 41$ tes. Balaenoptera? Il Prof. Bellardi rinvenne pure molti anni addietro al Pic Brusà, presso Pino, ancora sulle colline di Torino, delle reliquie di ossa fossili che egli suppose fossero di vertebre e, dalla loro compatta natura, forse di Cetaceo. Esse erano poi talmente in cattivo stato da non potersi nemmanco assicurare con certezza e della appartenenza alla classe dei Cetacei e della vera posizion loro nello scheletro, e vennero per conseguenza abbandonate. 
avanti allo indietro, or dallo indietro in avanti. Ai lati sono abbastanza visibili tre lieri strozzature che corrispondono colla metà lunghezza di tre singole vertebre, e fra queste, in corrispondenza della saldatura di due vertebre consecutive, osservo, al margine superiore dei fianchi, delle pieghe ossee trasversali sporgenti da uno fino a tre millimetri e continuantisi anche sulla faccia superiore nel solco lasciato fra le origini degli archi neurali successivi.

Notiamo infine che tutta questa faccia infero-laterale è costituita come da un guscio osseo, compatto, molto più denso della materia ossea avriluppata ed arente uno spessore variabile fra i quattro ed i cinque millimetri, ora strettamente aderente alla materia spongiosa sottostante, or mascherante delle carità che non risultano allo esterno che per lo sfondamento della lamina compatta in tali punti di minor resistenza alle forze che dallo esterno vi agirono contro. E notevole anzi che in un punto collocato a sinistra tra il secondo ed il terzo corpo di vertebra conservati, una di tali cariti non sia stata ricoperta dalla lamina ossea, arendo questa invece lasciata una bottoniera beante ed a bordi arrotondati misurante $21 \mathrm{~mm}$. di altezzi per 13 di lunghezza.

La faccia superiore del pezzo è la più istruttiva. In essa noi vediamo: ai lati l'origine di tre archi neurali successivi ed il fondo del canale neurale occupante i due quinti mediani della larghezza. Esso è collocato non già orizzontalmente, ma con lieve pendio ascendente dallo avanti allo indietro fino al terzo anteriore dellà terza vertebra conservata, e di li ridiscendente allo indietro. Il quarto corpo di vertebra è rotto e non ne possiamo verificare nè la lunghezza, nè l'altezza; egli è però certo che il suo spessore presente è ancora piu grande di quello della vertebra che lo precede. Lo stesso dicasi della prima vertobra che abbiamo dinanzi; il suo corpo è pure rotto anteriormente, quindi non possiamo dire precisamente quale ne fosse lo spessore; sappiamo però che esso è ancora presentemente superiore di quello della terza, ed a più forte ragione, di quello della seconda.

Le pieghe ossee sporgenti che notai alla parte superiore dei fianchi e che dissi essere le ultime traccie della saldatura di due vertebre consecutive, dopo essersi per breve tratto ripiegate sulla faccia superiore della sinostosi, vanno rapidamente obliterandosi ed il loro prolungamento è sostituito da un solco che attraversa tutto il canale neurale. Soventi la lamina ossea compatta costituente il fondo di tali solchi è abbastanza sottile per rompersi molto facilmente ed allorchè io, dopo avere collo scalpello esportata molta della roccia che ricopriva il fossile, sfondai due di questi solchi, ciò che del resto era già in parte arrenuto all'epoca dellia sepoltura del pezzo, trovai che mentre al di fuori la lamina ossea della sinostosi dimostrava una fusione completa dei diversi corpi di vertebra l'uno coll'altro, al di dentro non era affatto così ogni vertebra ha difatto la sua brava epifisi anteriore e la posteriore e, fra due epifisi adiacenti, una carità a mo' di disco, occupata in vita da una cartilagine intervertebrale e nello stato presente da sabbia indurita che aveva trovato per sfondamento della lamina compatta esterna un passaggio a venire ad occupare quei rani. Constatai tale fatto fra la prima e la seconda e fra la terza e la quarta delle vertebre presenti; stimai opportuno di non guastare il contatto fra la seconda e la terza dove la superficie superiore era un po' meglio conservata. 
Per ciò che si riferisce agli archi neurali poco arremo da aggiungere. La prima vertebra che abbiamo dinnanzi non mostra che l'origine del pedicolo destro. Pare che questo nel sollevarsi dalla base, lunga quant'era la lunghezza della vertebra, si accorciasse rapidamente in una lamina verticale arente un centimetro e mezzo di diametro longitudinale su due centimetri e mezzo di diametro trasversale, lasciando tra sè ed il pedicolo della vertebra successiva un foro di coniugazione il cui diametro antero-posteriore era maggiore di quello di ciascun peclicolo. Il corpo della vertebra seguente è più sottile del primo e mostra l'origine dei due pedicoli; essi son collo. cati quasi trastersalmente alla direzione del frammento, con lieve inclinazione allo indietro ed allo indentro. La lunghezza del loro dinmetro antero-posteriore è quasi eguale a quella del diametro dell'intiero corpo di vertebra compreso tra l'epifisi anteriore e la posteriore e la larghezza di ciascun pedicolo è di circa tre centimetri e mezzo. Staccatisi dal corpo della vertebra, dovevano i pedicoli allargarsi in fuori per dare origine all'apofisi trasversa superiore della. quale non resta altra traccia.

La terza vertebra, più lunga che la seconla, manca del pedicolo sinistro che è troncato alla base, presenta il destro meno appiattito dallo avanti allo indietro che non quelli della vertebra precedente. La sua faccia anteriore fa colla direzione generale della sinostosi un angolo aperto verso l'avanti e l'indentro piu saliente di quello che notammo per la vertebra precedente. Il pedicolo, elevatosi dal fondo del canale neurale di circa un centimetro, si ingrossava tanto allo avanti che allo infuori, sia per saldarsi probabilmente coll'arco neurale della vertebra precedente che per dar luogo al processo trasverso superiore. La faccia posteriore poi di tale pedicolo è quasi trasversale e, per ciỏ che se ne vede, pressochè verticale, lasciando dietro di sè un foro di coniugazione alto e breve.

La quarta vertebra è rotta troppo al disotto del fondo del canale neurale per mostrarci ancora traccia di questi pedicoli.

Dato cosi un rapido sguardo alle parti conservate, cerchiamo di collocare in sistema questo interessante frammento.

La natura dell'osso lo inclica come avente appartenuto ad un animale decisamente acquatico e piủ precisamente a qualche Cetaceo. Oltracciò passando in rivista i casi conosciuti di fusione di vertebre nei Mammiferi terrestri viventi o fossili troviamo sempre in essi maggiore riduzione del corpo delle vertebre e maggior sviluppo ed abbassamento dei processi trasversi di quello che noi abbiamo incontrato nel caso presente. Ancora, nei Mammiferi terrestri le vertebre sono sempre allo esterno perfettamente discernibili fra loro, anche qualora ne venisse offerto in esame un frammento ridotto nello stato del presente, mentre qui non possiamo distinguere un tal fatto che allorquando noi penetriamo con tagli nello interno della sinostosi. Ammesso dunque che trattisi di vertebre di Cetaceo, noi non abbiamo per quest'ordine di Mammiferi altro esempio di coalescenza fra le vertebre successive che nella regione cervicale e nella estremità della regione caudale (questo secondo caso non merita però nella presente questione di venir considerato, vista la forma e la riduzione delle parti che distinguono sempre le vertebre caudali dei Cetacei).

Ed io ho veramente considerato come parte di regione cervicale di un Cetaceo tale sinostosi, ed in essa parmi dover riconoscere la $5^{\mathrm{a}}, 6^{\mathrm{a}}$ e $7^{\mathrm{a}}$ vertebra cervicale e la $1^{\text {a }}$ dorsale. 
Questa regione cervicale comparata con quella delle Balene distinguesi a primo colpo d'occhio per la sua forma; infatti una sezione trasversale all'asse condotta per una regione cervicale di Balena in corrispondenza della $5^{\text {a }}$ vertebra mostrasi, per la parte che corrisponde al corpo, per lo più subquadrangolare e quand anche in alcune specie di Balene manchino già in tale posizione $\mathrm{i}$ processi trasversi, tuttavia essi vi sono rappresentati da lievi rigonfiamenti in corrispondenza dei due angoli inferiori della sezione. Qui invece la faccia inferiore della sinostosi è a fondo di battello ed in qualunque punto si conduca una sezione trasversale alle vertebre essa non sarà mai angolosa in basso nè mostrerà mai traccia alcuna di apofisi trasverse inferiori da essa incontrate.

La sinostosi cervicale delle Balene è in generale munita inferiormente, lungo la linea mediana, specialmente per le vertebre collocate più allo indietro, di una lieve carena semplice o doppia che qui manca affatto. Ma niuna Balena possiede nella sua sinostosi cervicale alcuna vertebra successiva allo atlante, il cui sviluppo longitudinale corrisponda, a parità di dimensioni, a quello delle vertebre che abbiamo dinanzi, nè finalmente mi consta che sezioni longitudinali condotte pel piano di simmetria di tali sinostosi abbiano dimostrata la presenza di una individualizzazione delle singole vertebre tanto spinta quanto la vediamo nel caso presente.

Nè la forma dei corpi di vertebre permettendomi di ravvicinare il mio pezzo ad alcuno dei generi intermedii fra le Balene e le Balenottere, ho voluto estendere le comparazioni ai Cetodonti: infatti losservazione della regione cervicale del genere Physeter pare mi abbia fornito i dati necessari ad una sicura collocazione del pezzo in questione.

Se la sinostosi nella quale sono comprese, nei viventi Fiseteridi, le sei vertebre cervicali consecutive allo atlante si mostra spinta talora a tal grado di fusione delle parti che una sezione praticata pel piano di simmetria più non mostra alcuna traccia della primitiva individualità delle singole vertebse, tuttavia la figura delle sezioni trasversali praticate in corrispondenza delle due o tre ultime di esse cervicali è già molto piu vicina a quelle che otterremmo segando in egual modo il nostro pezzo.

Infatti la carena inferiore delle ultime cervicali del Physeter macrocephalus, invece di essere puramente localizzata alla linea mediana della faccia inferiore, si espande ed acquista notevole estensione occupando tutta la faccia inferiore stessa e parte delle laterali; i processi trasversi inferiori poi, ancora, tubercoliformi in corrispondenza della terza cervicale, sono nelle successive completamente obliterati ed il loro punto di origine non sarebbe più segnato che da un lieve angolo arrotondato al punto di confine fra le faccie laterali e l'inferiore della sinostosi.

Il corpo della sinostosi del Physeter, in corrispondenza delle cervicali posteriori, viene in tal modo ad acquistare nella sezione trasversa una figura cordiforme, depressa e già molto comparabile a quella che troviamo nel pezzo nostro e noi non abbiamo che da arrotondare alquanto di più la faccia inferiore e ad estenderla, per ulteriore obliterazione delli angoli infero-laterali, sui fianchi per venire ad ottenere dalla sezione trasversale delle ultime cervicali di Fisetere una figura perfettamente analoga a quella che dà il pezzo nostro.

Rimane ancora la men comṕleta fusione dei corpi delle vertebre ed il loro maggiore sviluppo longitudinale. Sappiamo che nella regione cervicale dei Fiseteridi lé 
parti, completamente indistinguibili nella sezione mediana della sinostosi dello adulto si possono però riconoscere nel giovane. Un tale giovanile carattere fu riscontrato in un pezzo trovato dal Lebréton (1) e proveniente probabilmente dal Crag di Anversa, dove una sinostosi che ai caratteri suoi principali fu giudicata comparabile alla sinostosi cervicale dei Fiseteridi e che allo esterno mostrava completamente fusi in uno i corpi di quattro vertebre, allorchè venne segata dimostrò perfettamente clistinti e riconoscibili gli elementi che costituirano la sinostosi.

Basandoci sorra un tal fatto e supponendo la fusione degli elementi ancora meno spinta che in questo ultimo caso, tantochè, oltre a restar distinto come un solo pezzo il complesso di una diafisi e di due epifisi, restino ben differenziate e riconoscilbili non solo le diafisi ma fra due diafisi consecutive trovino modo di svolgersi e restare distinte anche le epifisi, ammettendo per spiegare un tal fatto un minore schiacciamento antero-posteriore delli elementi cervicali l'uno sull'altro e di naturale conseguenza maggiore allungamento di ciascuno di essi ed una probabile oscura mobilità nella vita fetale, noi saremo di leggieri portati a figurarci una regione cervicale quale ci sta dinanzi agli occhi col pezzo in questione, dove i corpi delle vertebre successive poterono presentare tre punti distinti di ossificazione per ciascuno, mentre la parete esterna di ciascuna molto più rapidamente sviluppandosi e confondendosi con quella delle vertebre adiacenti, le rinchiudera in un astuccio osseo, uniforme, senza lasciare altra esterna traccia della presenza di più elementi che le lievi ripiegature ossee gia segnalate.

Ho detto in principio che la quarta vertebra che arevo dinanzi era da me ritenuta quale prima della serie dorsale. Le ragioni principali di questo mio modo di vedere sono le seguenti: io considero come settima cervicale la vertebra che gli sta immediatamente dinanzi, per avere un corpo molto più lungo della precedente o seconda. per avere i pedicoli che portavano l'arco neurale molto piu forti delle precedenti e perchè il fondo del canale neurale a piano inclinato allo aranti nelle vertebre cervicali precedenti arriva, sul terzo anteriore della stessa, alla linea di sua elevazione massima ridiscendendo lievemente o mostrandosi quasi orizzontale pei due terzi posteriori.

Questi caratteri concordano con quanto osservo nei Cetacei, la cui sinostosi cervicale si estende alla settima vertebra e, fino ad un certo punto, anche in quelli nei quali essa non è cosi spinta, anzi è carattere quasi generale che tutte le apofisi della settima cervicale sono molto più sriluppate di quelle della sesta sia pei Cetacei a settima cervicale saldata, sia per quelli dove essa è libera e molto soventi tali apofisi sono, almeno in parte, assai più sviluppate nella settima cervicale che nella prima dorsale.

Ammessa come settima cervicale una vertebra, ne viene di conseguenza che la successiva debba essere la prima dorsale, anche allorquando, come nel caso presente, essa sia in tanto cattivo stato ridotta da divenire irreconoscibile di per sè sola. In appoggio però al mio modo di vedere ricavo ancora da essa il carattere della sua molto maggiore lunghezza; infatti la vertebra che rengo di considerare come settima cervicale misura dallo avanti allo indietro, e comprese le epifisi anteriore e posteriore,

(1) L'Homocetus Villersii di cui si parla nella Ostéographic des Célacés vivants el fossiles di GrnvalS o VAN Beneuen, pag. 332, che è disegnato a tav. XX, fig. 15 dell'atlante della stessa opera e che nella spiegazione delle tavole dello atlante viene pure chiamato Prophyseler cervicalis. 
$45 \mathrm{~mm}$., mentro questa, che è rotta posteriormente prima di poter mostrare alcuna traccia della faccia di adattamento all'epifisi posteriore, misura già $55 \mathrm{~mm}$. di lunghezza; volendo noi a tale lunghezza aggiungere, e con tutta l'avarizia possibile, quella che manca, non potremo dar meno di un centimetro per la restante parte della diafisi e per tutta l'epifisi posteriore, e non ne risulterà per la intiera vertebra meno di $65 \mathrm{~mm}$. dallo avanti allo indietro in confronto coi 45 della vertebra precedente, cioè quasi la metà della lunghezza di essa in più.

Considerata come prima dorsale la quarta vertebra riconoscibile nel nostro pezzo, ci si para dinanzi un altro fatto assai importante, la fusione cioè della prima vertebra dorsale nella sinostosi cerricale. Tale fatto, conosciuto gia da tempo sia per Balene virenti (la Balena di Lacèpède, Balaena Vun Benediana Capeltin del Museo di Parigi) sia per Balene fossili (la Baluena etrusca di Capellini), non è però, a quanto mi consti, stato finora segnalato per alcun genere di Cetodonti.

Per conseguenza, al modo stesso in cui molti caratteri distintivi di generi di Misticeti vengono a trovare $\mathrm{i}$ loro corrispondenti nei Cetodonti, anche il fatto della saldatura della prima vertebra dorsale nella sinostosi cervicale, che è più o meno spinto in alcune specie di Balene, non mancherà d'ora innanzi neppure del suo corrispondente fra i Cetodonti.

Ho già detto della probabiliti che l'animale cui appartenne la regione cervicale di cui ci siamo fin qui occupati debba essere compreso in un genere vicino ai Fiseteridi. I suoi caratteri però, pure portando a questa conclusione, non permettono mai di considerarlo come una specie del genere Physeter stesso. Anche il genere Homocetus di Anversa, pure ammettendo che la regione cervicale designata con tal nome abbia appartenuto ad un Fiseteride, ciò che nemanco il Gervais potè assicurare, non può comprendere questo nostro fossile come una seconda specie. Le parti di altri Cetacei fossili riferiti a generi vicini al vivente Physeter non sono comparabili colla nostra: mi reggo adunque costretto a dover dare un nuovo nome generico al nostro fossile proponendo l'appellativo di Priscophyseter o di precursore dei Fiseteridi e nominando la specie come prima del genere coll'aggettivo di typus.

Arremo dunque a notare fra i Cetodonti il nuovo genere Priscophyseter coi seguenti caratteri :

Regione cervicale allungata, corpi deble vertebre confusi allo esterno, distinti allo interno e muniti di epifisi, a sezione trasversale semi-ellittica, sforniti completamente di apofisi trasverse inferiori; - la prima vertebra dorsale congiunta alla sinostosi cervicale.

I fossili che verrò descrivendo nei due numeri successivi, appartenendo pure a Fiseteroidi, confermeranno la presenza di questa famiglia fra i fossili pliocenici dell'Alta Italia e daranno quindi, io spero, maggior probábilita alla mia collocazione della sinostosi in questione fra $\mathbf{i}$ membri di una famiglia che fino ad ora non areva lasciati residui conosciuti nei nostri terreni.

Nota. - Il Van Beneden, nel suo passaggio a Torino, ebbe occasione di vedere la sinostosi cervicale che veniamo di esaminare; egli ne parlò nel suo lavoro: La Balcine fossite du Mrusée de Milan che ho più sopra citato e la indicò siccome regione cervicale di una «Balacnula malata». Spiacemi di essere su questo punto di opinione diversa. 
da quella dello illustre Cetologo belga, ma l'esame dei pochi caratteri che il fossile presenta, che ho più volte e con somma attenzione ed imparzialità cercato di studiare e che son venuto esponendo nelle pagine precedenti, mi obbligò a ritenere il fossile piuttosto per un Cetodonte che per un Misticeto. Forse altre scoperte scioglieranno la questione el io sarò allora felice tanto di arer aruto ragione quanto di confessare di aver mal visto ed interpretato un pezzo per ora ben restio ad una esatta determinazione.

Similmente il Van Beneden ha riconosciuto che la famosa sinostosi di Homocetus Villersii invece di avere appartenuto ad un Fiseteride spettava ad una Balacmula. La comparazione che abbiamo fatta nelle pagine precedenti della sinostosi di Priscophyseter con quella dell'Homocetus era limitata al solo fatto di una piu o men progredita fusione dei corpi di vertebre senza aver molto riguardo che ciò avrenisse in un Misticeto od in un Cetodonte, quindi, anche quando uno dei pezzi di comparazione alsbia dovuto successivamente essere traslocato di sottordine, ciò non implica che debba di naturale conseguenza venire traslocato il pezzo comparato. Noi abbiamo del resto fin da principio fatto osservare le differenze di forma che esistevano fra i corpi di vertebra componenti la sinostosi dell'Astigiana e quelli, prima dei Balenidi in generale, poi dei generi intermediarii fra i Balenidi ed i Balenotteridi, intendendo appunto per intermediarii i generi Balaenuta e Balrenotus.

\section{Genere HOPLOCetus Gerv.}

\section{N. 43. Hoplocetus minor PorTss.}

$$
\text { Fเง. } 87-90 \text {. }
$$

Fra i pezzi facenti parte dell'antica collezione del Museo ho trovato il magnifico dente rappresentato (fig. 87-88): esso portava l'indicazione: «Dente di Squalodon, valle Andona, terreno pliocenico marino ».

Allorchè il Prof. E. Sismonda aveva voluto fare l'iconografia dei Vertebrati fossili del Piemonte, egli areva anche tenuto in considerazione questo dente e la sua figura si trova col N.6 in una tavola preparata per l'Accademia delle Scienze di Torino e che rimase sgraziatamente inelita.

Al N. 7 di questa stessa tarola era stata disegnata la sezione trasversale di un secondo dente eguale al primo ed appartenente allora alla Collezione tella R. Scuola di applicazione per gli Ingegneri in T'orino.

Molti anni dopo, nel 1873 , il Prof. Gastaldi faceva eseguire un nuovo disegno del dente intiero (N. 6 della tavola Sismonda), unitamente a quello di un altro dente che egli possedeva nella propria Collezione e li inviava, accompagnati da una copia della tavola Sismonda, al Prof. Brandt di Pietroburgo. Questi pubblicava i due disegni a tar. V (fig. 13 e 14) delle sue Ergacnzzngen zu den fossilen Cetaceen Europa's ed a pagina 49 del testo notara, pel primo, una tal quale rassomiglianza collo Hoplocetus crassidens GERv., e pel secondo, altrettanto coll $H$. curvidens GERv.

Riuniti i Vertebrati fossili raccolti dal Prof. Gastaldi a quelli del Mluseo Geologico di Torino, trovai fra di essi e il dente sezionato del disegno fig. 7 nella tavola Sismonda, e quello della fig. 14 nella tav. V del Brandt, ed infine 13 altri denti e frammenti

11 A. Portis. 
di denti provenienti dalla antica Collezione Sotteri (come del resto anche gli altri tre), dei quali, occupandomi dei Cetacei fossili del Piemonte, doretti intraprendere lo studio.

$\mathrm{Ne}$ risultò che, in questo piccolo numero di denti, dovetti tosto distinguere due forme principali, nettamente diverse l'una dall'altra e che molto bene si adattavano ad essere collocate in due genrri differenti. Per l'una potei trovare un posto conveniente in sistema col farne una nuova specie di un genere già conosciuto; per l'altra dovetti invece creare un genere nuovo, non essendovene fra gli antichi alcuno che la potesse comprendere.

I denti che appartengono al primo gruppo sono:

a) Quello completo stato figurato, ma senza determinazione, dal Sismonda E. nella fig. 6 della sua tavola inedita, disegnato poscia dal Gastaldi per conto del Brandt e da questi riprodotto (tav. V, fig. 13 e $13 a$ delle sue Ergänzungen) e comparato all'Hoplocetus curvidens.

b) Il dente sezionato stato figurato dal Sismonda nella stessa tavola del precedente alla fig. 7. Trovai le due metà del dente, ma esso manca della corona.

c) Il dente stato disegnato dal Gastaldi per conto del Brandt e da questi riprodotto (tav. V, fig. 14 delle sue Ergacnzungen) e comparato allo Hoplocetus crassidens. Anche questo dente manca della corona.

(d) Quattro corone di denti isolate dalla loro tuberosità radicale e riferibili, a quanto pare, alla stessa specie dei precedenti.

Tutti questi denti o frammenti paiono essere stati trovati nella medesima località in Valle Andona, i tre piu grandi appartennero alla Collezione Sotteri; probabilmente allorchè questa passỏ al Municipio il Sismonda E. ottenne di potersene pigliar uno pel Regio Museo. Le corone isolate pervemero in mano al Prof. Gastaldi in due epoche diverse (una volta tre ed un'altra una). Ora tutti si trovano riuniti nella Collezione del Museo.

I denti riuniti in questo gruppo sono distinti da una corona insensibilmente curva, di altezza variabile pei diversi denti, secondo che essi sono più o meno spuntati dall'uso e secondo la loro posizione nella serie mandibolare. Per una quasi intatta ho trovato $25 \mathrm{~mm}$., per un'altra molto usata alla punta, $18 \mathrm{~mm}$.; il diametro alla base della corona oscilla per i denti diversi fra i 13 ed i $14 \mathrm{~mm}$.

Lo smalto che riveste la corona è liscio verso la punta, sensibilmente rugoso in senso verticale più in basso; lo spessor suo, pressochẻ dappertutto eguale, è un po' più di un mezzo millimetro, senza però giungere mai ad un millimetro intiero.

Tali corone sono collocate ad angolo apertissimo sopra una enorme radice tuberiforme che presenta alla sua parte superiore un lieve restringimento a mo' di collo: anche questa parte, che avrà un centimetro di lunghezza e che continua la direzione della corona, è lievemente inclinata per rispetto all'asse della radice restante. A parte un tal collo, la radice è enormemente ingrossata per l'accumulazione irregolare di cemento attorno all'avorio del dente. Essa sarebbe fatta a cono assai allungato e rovesciato colla estremità smussata e perforata da una lieve cavità nutritizia che non si interna nel dente di più di un centimetro e mezzo.

Il dente più completo $(a)$ che io tengo dinanzi ha $95 \mathrm{~mm}$. di lunghezza; di questi ne spettano 82 alla radice; il suo maggior diametro, sopra alla metà della lunghezza, è di $33 \mathrm{~mm}$, la sua circonferenza di 103 . 
La sezione trasversale praticata a metà circa lunghezza della radice di un altro dente (b), misurante $33 \mathrm{~mm}$. di diametro massimo, mostra, partendo dal centro, un primo cilindro irregolare di avorio di circa $4 \mathrm{~mm}$. di raggio, stratificato concentricamente e non perforato al centro; questo rivestito da un secondo cilindro di avorio a struttura concentrica distintissima. Il raggio di questo secondo cilindro sarebbe di $8 \mathrm{l} / 2$ millimetri, dai quali dobbiamo dedurre i 4 spettanti al primo: abbiamo dunque per la corona circolare $4 \frac{1}{2} \mathrm{~mm}$. di raggio. Il doppio cilindro di avorio è rivestito di un forte strato di cemento a struttura fibro-raggiata e di tinta molto più chiara, discernibile quindi a Tprima vista dai due cilindri concentrici di avorio. Questa forte parete, misurante in diversi punti da 5 a $7 \mathrm{~mm}$. di raggio, si mostra, dove è più spessa, scomposta anche essa in due strati concentrici di eguale spessore ed è rivestita allo intorno da un invoglio irregolare di un mezzo millimetro di spessore e che non è altro che cemento più giovane non avente ancora assunta la struttura fibro-raggiata di quello interno.

Occorre notare un'ultina particolarità : il dente completo $(\alpha)$ presenta in un punto, alla parte superiore della sua grossa radice, una lieve depressione a grondaia, discendente obliquamente, ma presto interrotta. A questo non volevo da principio dare importanza, tuttochè glie ne abbia voluto dare, a quanto pare, il Sismonda che nel suo disegno la fece molto esagerare per renderla visibile. Il Gastaldi (e per conseguenza il Brandt) la trascurò affatto, e tale era, come dissi, anche il mio parere dapprima, poichè parevami che in mezzo alle altre irregolarità nella deposizione della parte esterna del cemento trovasse benissimo sua spiegazione anche quella solcatura. Ma l'altro dente $(c)$ stato disegnato dal Gastaldi e riprodotto dal Brandt (quantunque quest'ultimo non ricordi punto il fatto che nel suo disegno stesso è evidentissimo) presentara lo stesso fenomeno molto più spiccato. Omologhe alla impressione segnalata nel primo dente si osservano in questo secondo due impressioni, l'una più superficiale collocata nella porzione del dente che dicemmo rassomigliare ad un collo ed interessante, a quanto pare, ancora un po' la corona che qui è rotta e mancante completamente. Tale prima impressione, più profonda che nel caso precedente, ha una forma irregolare ed una superficie interna quasi liscia; essa è diretta obliquamente allo ingiù. L'altra impressione, collocata quasi diametralmente in opposizione alla prima, pare abbia cominciato a mostrarsi allo stesso modo di quella, cioè sul collo, interessando anche la base della corona; essendosi poi maggiormente estesa ed approfondita, die' luogo ad una carità collocata verticalmente, interessante almeno un quarto della lunghezza totale della radice ed aperta allo insủ ed all'infuori. La superficie di rottura da cui manca la corona del dente dimostra che questa per effetto delle due accennate impressioni venira poi ad avere alla base una sezione irregolarmente triangolare a lati arrotondati, dei quali uno convesso e due concavi.

L'esame del dente ci dimostra infine che le due impressioni non vennero prodotte durante il tempo di fossilizzazione del pezzo, nè durante quel periodo in cui per la scomposizione del cadavere i denti potevano giacere liberamente sul fondo marino ed esposti quindi allo insulto dei molteplici organismi litofagi, ma che esse si produssero invece sicuramente durante la vita dello animale cui i pezzi esaminati appartennero. Ciò constatato, tali impressioni non potevano avere che due spiegazioni: o l'incontro. di altri denti dal mascellare opposto od un caso patologico. 
Se si fosse trattato soltanto del dente figurato dal Sismonda, la prima spiegazione avrebbe potuto arere un'ombra di probabilità (in tal caso non avrei più potuto certamente colloc.re il fossile fra i Fiseteroidi, benchè la sua struttura additasse una tale analogia, e arrei doruto cercargli un posto fra i Cetodonti portanti denti superiormente ed inferiormente); ma la semplice osservazione delle impressioni visibili sul secondo dente distruggeva l'ammissibilità di tale ipotesi. Infatti due Centi successivi del volume e della forma dei presenti non aviebbero potuto essere impiantati in un mascellare in vicinanza reciproca tale da poter produrre le impressioni visibili sul dente $c$ ) alla sola. distanza in cui noi lo vediamo senza essersi confusi in un corpo solo, quinli, invece di due, avrebbero prodotta una sola delle impronte in questione; nel caso estremo poi che due denti consecutivi fossero giunti per reciproca divergenza delle due parti radicali a portare le due corone a questa sola distanza, le impressioni sul dente che le subi avrebbero dovuto essere convergenti invece che verticali; e l'atto della masticazione (o meglio, della presa dell'alimento) portando il contatto fra i due mascellari opposti ed $\mathbf{i}$ due denti che li guernivano in più direzioni e secondo variabili inclinazioni arrebbe, anzichè produrre gli effetti osservati, piuttosto decoronato rapidamente il dente e consumatane parte della radice in modo e direzione molto diversi da quel che vediamo.

La natura e la superficie di queste caviti ci dimostrano invece che dobbiamo cercar l'origine loro in una molto più probabile malattia della sostanza ossea (cementosa) stessa, in una carie prodotta da qualsiroglia causa, anche estranea; e che dopo arer corrosa e perforata la parte piu tenera e cementosa, strisciava lungo il cilindro eburneo più duro e resistente corrodendo pure profondamente quello. La mancanza, nel punto attaccato, di cavità polposa faceva si che il dente non ammalasse che localmente, che non cadesse per conseguenza dallo alveolo e che le parti molli circostanti aressero una qualche influenza nello arrotondare e rendere meno incomoli i margini lasciati troppo vivi e sporgenti dalla corrosione locale della materia cementosa.

Ammessa la causa patologica di tali cavità e come quindi esse non debbano venir tenute come caratteri attinenti alla forma originale dei denti, non ci rimane che di passare ai confronti.

Ho esaminati i caratteri dei denti dei generi Physodon, Palaeolelphis, Scaldicetus ed Hoplocetus, i quali tutti starebbero, parmi, molto bene riuniti in un genere solo al quale si potrebbe conservare il nome il più antico. Non voglio però insistere su di ciò e mi limito ad osservare come il dente $($ ), il più completo, offra grandissime analogie con quello di Physodon leccense GERr. del Miocene di Lecce, rappresentato nella fig. 16, tav. XX dell'opera del Gervais e Van Beneden (con esso concordereblue pure ad un di presso per le dimensioni), con quello del Palaeodelphis fusiformis GERv.(Du-Bus) del Crag di Anversa, disegnato fig. 19 della stessa tavola, il quale ha però dimensioni doppie del nostro, con quello dello Scáldicetus Carettii Du-Bus del Crag di Anversa della fig. 24 della tavola, il quale ha di nuovo dimensioni molto più grandi del nostro, ed infine con quello dell'Hoplocetus curvidens GERv. del Pliocene di Montepellier della fig. 25, stessa tavola, e della fig. 12, tav. III della Zoologie et Palcontologie Française del Gervais. Anche per questo le dimensioni sono molto superiori a quelle del nostro. Le maggiori analogie però si manifestano pei denti del Palacodelphis fusiformis ed ancor più per quello dell'Hoplacetus curvidens. 
Sarei per conseguenza di parere di considerare il dente in questione come arente appartenuto al genere Hoplocetus e ad una specie distinta per alcune varieta di forma e sorratutto di dimensioni dalle due specie già note di questo genere, cioè l'H. curvidens e l'H. crassidens.

Il Brandt paragonò il dente in questione piuttosto coll'H. crassidcris che col curvidens, ma dietro a disegni forse un po' esagerati: quanto poi al dente $b$ ), che il Brandt stesso paragono coll' $H$. curvidens, ed a mio debole parere con ragione, parmi che le differenze che lo distinguono dal dente $a$ ) siano di tal natura da non bastare a tenere i due separati e ciò tanto più quando si dia il doruto peso alle modificazioni arrenute per causa patologica che segnalai. Per conseguenza tanto il dente $a$ ), che il $b$ ). che il c), che quelli d) debbono, secondo me, venir riuniti in una sola specie che, avuto riguardo alle dimensioni minori in questa che nelle due specie gia note, propongo chiamare Hoplocetus minor.

\section{GeNere PHYSOTHERIUM PORTIS.}

\section{N. 14. Physotherium Sotterii PorTIs.}

Fis. 91-94.

Già dissi in pria come nella Collezione Gastaldi fossero compresi molti denti di Cetaceo, dei quali avero fatti due gruppi, quelli del primo appartenenti al genere c specie precedenti e quelli del secondo meritevoli di una descrizione a parte.

Fanno parte di questo secondo gruppo sette denti più o meno maltrattati, l'uno dei quali sezionato longitudinalmente e tre frammenti abbastanza insignificanti. Tutti provengono dalla antica Collezione Sotteri e sono stati trovati nei terreni pliocenici dell'Astigiana, probabilmente in Valle Antona.

Le corone di questi denti, tutte mancanti della sommità, sono irregolarmente coniche, a sezione ellittica; la parte basale che ne resta di queste corone ha ancora più di due centimetri di altezza, l'apicale che manca non ne poteva avere meno di uno, totale per la corona intera quasi tre centimetri e mezzo di altezza per $27 \mathrm{~mm}$. di maggiore e 23 di minor diametro alla base. Il margine inferiore della corona non è normale all'asse, ma fortemente inclinato dal lato interno del dente. Lo smalto che riveste la corona, liscio verso l'apice, trasversalmente rugoso presso la base, ha circa un mezzo millimetro di spessore.

Queste corone sono inserite ciascuna sopra una grossa radice anch'essa conicoellittica ma più appuntita, per modo che le basi dei due coni vengono ad adattarsi l'una contro l'altra e da questo punto, che corrisponde circa ad un terzo della lunghezza dello intiero dente e che ne segna la zona di massimo rigonfiamento, si eleva allo insù il cono ottuso della corona e si abbassa dall'altra, facendo col primo cono un angolo molto ottuso, quello più appuntito della radice.

Questo cono radicale è lievemente solcato longitudinalmente ed è rivestito di un forte strato di cemento che possiamo molto ben riconoscere in uno dei denti stato sezionato parallelamente all'asse e che arendo un diametro di $26 \mathrm{~mm}$. mostra un cilindro eburneo di $18 \mathrm{~mm}$. circondato da un astuccio cementoso di $4 \mathrm{~mm}$. di spessore. 
La cavita nutritizia piccola e ristretta non arriva più su della metà lunghezza del cono radicale.

Dai pochi cenni che precedono, e sovratutto dallo esame delle figure ben si può vedere quanto sia grande la differenza che corre sia nella forza che nello sviluppo relativo delle diverse parti fra $\mathrm{i}$ denti del precedente gruppo e quelli di questo, e come, anche data una grande latitudine alla variabilità nei denti di una stessa specie di Fiseteridi, secondo che essi tengono piuttosto una che un'altra posizione nella mandibola, $\mathrm{i}$ due gruppi non possano in alcun modo venir tenuti assieme in una specie sola.

Dirò di più, ammesso che $\mathrm{i}$ denti di cui presentemente ci occupiamo spettino realmente ad un Fiseteroide, cosa che dietro l'esame loro e della loro struttura interna parmi abbastanza ben dimostrato, la loro forma è tanto caratteristica che io non trovo alcun genere vivente o fossile di questa famiglia nel quale essi possano entrare a far parte; sono costretto a considerarli come appartenenti ad un nuovo genere al quale, per ricordare la posizione che occupa fra i Fiseteridi, propongo di dare il nome di Physothcrium, mentre la specie unica che presentemente lo costituisce desidero venga dedicata alla memoria dell'Abate Sotteri, per merito del quale ci vennero conservati questi interessantissimi denti.

In conclusione, se per mezzo di un frammento di scheletro parci aver messa in sodo la presenza della famiglia dei Fiseteridi nei terreni pliocenici del nostro Piemonte, per mezzo dei denti potemmo invece dimostrare la presenza di almeno due generi spettanti a questa famiglia. In quale rapporto poi stiano fra di loro denti e scheletro, se quest'ultimo spetti piuttosto all'uno o all'altro o a nessuno dei due, è cosa per ora impossibile a definire, le parti non essendo in nulla fra loro comparabili ed essendo state trovate in diversi tempi, in diverse località.

\section{FAMIGLIA DEI TIFIIDI}

\section{Genere BERARDiopsis Portis.}

\section{స. 40. Berardiopsis pliocaenus PORTIS.}

FiG. 95,96 .

Le Collezioni paleontologiche del IIuseo di 'T'orino comprenderano già da molti amni una serie di ossa trovate nelle sabbie gialle dell'Astigiana e che, dopo essere state riconosciute quali vertebre di Cetacei, erano state trascurate siccome di poca importanza e lasciate nella Collezione ancora con tutta la sabbia che ad esse aderiva allorclıè giunsero in Museo.

Desiderando di renderle almeno visibili, mi diedi a ripulirle e ad isolarle dalla sabbia, procurando insieme di ricongiungere quelle che si trovavano in più pezzi ed al fine del lavoro potei mettere insieme una serie di circa quattordici vertebre o framnenti di vertebre che pigliavano abbastanza naturalmente posto l'una in seguito alle altre e che mostravano d'aver fatto parte della regione caudale di un Cetaceo. 
L'animale cui tali vertebre appartennero era, allorchè mori, ancora assai giovane; infatti le epifisi (che come sappiamo si saldano ai corpi dapprima nella regione caudale) non sono conservate in tutte le vertebre e quelle che le portano ci mostrano quanto lontano esse ancora si trovino da una completa saldatura.

Le vertebre caudali più completamente conservate mostrano un corpo subcilindrico coi seguenti caratteri: per le vertebre collocate più allo avanti il cliametro longitudinale del corpo è aguale al trasversale ed entrambi sono minori del rerticale; la nona della presente serie, ad esempio, misura dallo avanti allo indietro mm. 75 , trasversalmente alla faccia posteriore $75 \mathrm{~mm}$. e verticalmente alla stessa faccia $84 \mathrm{~mm}$. Progredendo verso la pima caudale queste relazioni si modificano e troviamo che il diametro trasversale diventa il minore; cosi nella vertebra duodecima della serie ricostituita troviamo il diametro longitudinale del corpo di mm. 71 , il verticale alla faccia posteriore di mm. 74 e quello trasversale alla stessa faccia di mm. 61 .

Le faccie articolari, nelle vertebre che ancora conservarono le epifisi, sono piane per la loro massima parte; la sola porzione centrale, il cui raggio è circa un terzo di quello dell'intiera facia, si mostra lievemente incarata e rugosa, e cio tanto per la faccia anteriore quanto per la posteriore di ciascuna vertebra. Dicemmo innanzi come la forma di ciascun corpo fosse pressochè cilindroide; conviene aggiungere però come le quattro faccie che costituiscono i fianchi di ciascun corpo, le due laterosuperiori cioè e le latero-inferiori, si presentino (specialmente per le vertebre collocate piu presso alla regione lombare) perfettamente individualizzate l'una dall'altra, e ciò non solo a causa dei diversi processi che le separano, ma eziandio per la quasi rettilineità di direzione di ciascuna; quindi una qualunque di esse, estesa oltre i presenti confini, non si confonderebbe colle contigue, ma le incontrerebbe facendo un angolo diedro. OItracciò ciascuna faccia mostrasi, in proporzione dei bordi anteriore e posteriore molto più sporgenti allo infuori, abbastanza scavata nella sua regione mediana. Tali caratteri vanno naturalmente perdendosi man mano che ci accostiamo alla estremità caudale, dove però se la faccia latero-superiore di ciascun lato tende a confondersi colla contigua latero-inferiore, rimane tanto piu distinta la direzione della curva di un fianco da quella del fianco opposto.

Egli è carattere abbastanza generale delle vertebre caudali dei Cetacei di venire attraversate di basso in alto dalle arterie intercostali, con questa avvertenza però che nei Misticeti le suddette arterie partendo dal solco emale e girando attorno al corpo della vertebra, allorché si trovano contro all'origine del processo trasverso, lo forano e passano cosi sulla faccia latero-superiore, e soltanto nelle ultime caulali gia sfornite di ossa a $V$ passano affatto nel corpo della vertebra, mentre negli Odontoceti le arterie traforano il corpo vertebrale piu allo indentro, piu presso al piano mediano, gia in regioni corrispondentemente anteriori.

Nel caso presente tali perforazioni raggiungono il massimo loro, sarei per dire, di internamento; difatti già nella regione caudale anteriore vediamo come l'arteria intercostale di ciascun lato si stacchi dal gran tronco arterinso collocato nel canale emale sotto un angolo acutissimo allo insù, tanto da entrare subito nel corpo vertebrale forando sotto al rilievo che dà, allo aranti ed allo indietro, origine ai tubercoli di articolazione per le ossa a $V$; come superato tale rilievo essa non si 
mostri a giorno che per una profonda incisione della faccia infero-laterale del corpo e dalla quale si staccano altre incisioni secondarie meno profonde, dirette in vario senso ed alloggianti arteriuscole minori che serrono a nutrire la regione periferica inferiore; come l'arteria principale seguiti il proprio percorso profondo nascondendosi un'altra volta molto al disotto della origine del processo trasverso e non riesca alla luce che al disopra della metà altezza nella faccia latero-superiore, donde, sempre alloggiata in profonda incisione, va poi a raggiungere il canale neurale al di dietro dell'origine dell'arco. Nelle caudali estreme, ma ancora fornite di ossa a $V$, la arteria di ciascun lato entra dal basso ed immediatamente dal canale emale nel corpo di ciascuna vertebra e non ne riesce che in prossimità dell'arco neurale, mentre un solo ramuscolo secondario si stacca ed esce per un forellino nella faccia lateroinferiore a nutrire la superficie della vertebra, che del resto si presenta eminentemente irregolare e rugosa.

L'arco neurale è, relativamente alla posizione occupata da queste vertebre nello scheletro, ancora molto sviluppato; anche l'ultima delle vertebre presenti ne mostra ancora le traccie. Quelle collocate più anteriormente dovevano portare un arco munito di un grande e forte processo superiore. Oltracciò è in esse molto ben distinta con una banda di maggiore rugosità la linea di saldatura di ciascun pedicolo dell'arco neurale al corpo della vertebra.

I processi trastersi sono essi pure sviluppatissimi e non si perdono che nelle ultime caudali, essendo costi rappresentati solo da maggior rugosità della superficie; finalmente sriluppatissimi sono pure i tubercoli inferiori per l'articolazione delle ossa a $V$. Come di regola, il paio posteriore di questi tubercoli è sempre molto più sviluppato del paio anteriore e tale relazione si conserva fin nelle caudali estreme. Debbo aggiungere come nelle caudali anteriori ciascun tubercolo anteriore sia unito con quello che gli sta dietro per mezzo di un rilievo osséo tagliente, dentro al quale passa il canale arterioso che or è poco descrivemmo, e come nelle posteriori tale rilievo te:Ida a scomparire e ciascun tubercolo anteriore ad isolarsi dal posteriore.

In complesso: tutti i caratteri presentati da questa regione caudale ci inducono a cercare fuori dei Misticeti un posto conveniente per collocare l'animale cui questa coda appartenne, e fra gli Odontoceti, nei soli Zifiidi noi troviamo delle vertebre che presentino caratteri eguali a quelli che abbiamo precedentemente descritti. Anzi trovo nel solo genere Berardius delle parti di scheletro che per dimensioni tanto assolute che proporzionali, per forma e per caratteri si possano comparare con quelle che abbiamo dinanzi. Io son quindi portato a credere che, al modo stesso col quale quasi tutti gli altri tipi di Cetacei ebbero già nel mare pliocenico i loro rappresentanti, cosi l'abbia pure potuto arere il genere Berctrdius, e quindi assegno per ora all'animale di cui ho dinanzi gli avanzi il nome generico di Berartiopsis e lo specifico di pliocacmus, augurandomi di trovare in seguito altri e migliori residui che mi risolvano a collocarlo direttamente nel genere Berardius (1).

(1) Ho trovato che alcune delle vertebre disegnate dal CAper.usi nella sua tavola IV annessa alla Memoria: Di un'Orca fossile scoperta a Cetona (V. Bibliogr., $N^{\circ} 74$ ), presenta appunto qualcuno dei caratteri qui indicati, principalmente la posizione e relazione dei fori per le arterie intercostali per riguardo al corpo della vertebra. Diver'sa è però la forma generale di ciascuna vertebra. 
Occorre arvertire che alcune vertebre di Berardiopsis pliocaems presentano in direrse posizioni dei tagli, i quali dimostrano l'azione esercitata da un nemico aggressore dell'individuo cui le vertebre appartennero. Ho gia ammesso in altri casi che tale nemico abbia potuto essere uno Squalide. I tagli presenti, la natura, posizione e forma loro non farebbero che confermarmi nella mia opinione.

\section{N. 4(i. Berardiopsis pliocaenus Portis.}

FiG. 96 .

Oltre alla serie di vertebre precedentemente descritta si sono ancor trovate nei terreni pliocenici piemontesi altri avanzi riferibili alla stessa specie. Ad esempio vidj nei magazzini del JIuseo un'altra vertebra caudale proveniente essa pure dall'Astigiana, e probabilmente dalle argille plioceniche, clie ha appartenuto ad un individuo adulto e che per dimensioni e per tutti i suoi caratteri eguali a quelli precedentemente enumerati mostra aver pure appartenuto al Berardiopsis pliocremus.

\section{N. 47. Berardiopsis pliocaenus PoRTIS - V. N. 17375.}

Anclie il Prof. Gastaldi raccolse nell'Astigiana una piccola vertebra caudale ed un frammento di un'altra. La vertobra intera mostra essere una delle ultime della serie, i suoi caratteri sono identici con quelli della corrispondente vertebra della serie caudale di cui parlai al N. 45, colla differenza che qui trattasi di un individuo adulto. Riferisco anche questi due pezzi alla specie Berardiopsis pliocnemes.

Anche la vertebra caudale intiera raccolta dal Gastaldi porta numerosi e profondi intagli doruti ai denti dei pesci cani.

\section{DELFINORINCHI}

\section{Genere SQUALOdon Grateloup.}

\section{N. 48. Squalodon Gastaldii J. F. Brandt.}

Ad arricchire la fauna cetologica del Piemonte non mancarono gli Squalodonti. Uno scheletro di un individuo di questa famiglia, benchè incompleto e frammentario, renne anni addietro scoperto nel calcare presso Acqui e dal Prof. Gastaldi raccolto e conservato nella Collezione del Valentino donde passò l'anno scorso a quella del R. Mruseo Geologico.

I pezzi principali di questo Squalodonte vennero, per cura dello stesso Prof. Gastaldi, fatti disegnare e le figure inviate all'illustre cetologo Brandt di Pietroburgo che nella sua grande opera: Untersuchungen ueber die fossilen und subfossiten Cetrceen Europa's, a pag. 326, descrisse come nuova specie lo Squalodonte d'Acqui dedicandolo al Gastaldi e ne diede i disegni (tav. XXXII).

12 A. Pontis. 
Nessun altro scheletro o frammento di Squalodonte essendo stato rinvenuto di poi in Piemonte, credo che il miglior modo per me di conciliare la completezza del Catalogo dei nostri Cetacei fossili con una buona descri\%ione di ciascuno di essi sia di riportare integralmente, allorchè ne $\dot{e}$ il caso e traducendola se occorre, la descrizione originale di chi primo descrisse una delle nostre specie. Cosi ho fatto per pezzi precedentemente menzionati, cosi faccio per lo Squaloclon Gastallii Braxd, cosi farò più avanti per lo Steno Gastaldii dello stesso autore. Ecco intanto il capitolo che presentemente ci interessa:

* Spec. ̈̈. Squalodon Gustulnlii? J. F. Brannt (tav. XXXII, fig. 1-23). Per " la bontà del sig. Prof. Gastaldi in 'Torino riceretti le eccellenti figure (non però " accompagnate da alcuna descrizione), riportate alla mia tavola citata, degli avanzi

" di uno Squntodon il quale renne scarato presso Acqui dagli strati inferiori

* della formazione mincenica media corrispondente alla Mollassa media dei Geologi « sqizzeri.

« Non potendo adattarmi a riferire con sicurezza questi avanzi a quelli di alcuna " delle specie già stabilite, riposanti per lo pun sopra resti ancor più incompleti, cosi ne " feci una nuova specie, dedicata al sig. Gastaldi, sulla esistenza della quale, siccome su " quella delle altre, naturalmente il solo futuro potrà portare una sanzione definitiva.

" Naturalmente per nuove ricercle arvenire dorranno essere tenuti presenti sia i pezzi

" qui piủ basso ricordati, disegnati dal Scilla, sia quelli descritti (vecli oltre) da Molin

" e da Suess.

"Gli avanzi consistono, per quanto si può riconoscere dalle tigure gentilmente " comunicatemi, in tre frammenti del mascellare inferiore (fig. 1-3), sei denti isolati

" (fig. 4-9), una vertelura cervicale (fig. 10 e $10 a$ ), una, certo delle posteriori, ver-

" tebra dorsale (fig. 11-12), un frammento di una delle vertebre corsali lè piu indietro

" (fig. 13, 14, 15), una vertebra lombare quasi completa (fig. 16), il frammento di

* una vertebra lombare (fig. 17-18), come pure due vertebre caudali incomplete (fig. 19,

* 20,21 e 22) e una costa abbastanza completa (fig. 23 ).

"La piccolezza dei denti isolati, disegnati al vero, e le dimensioni, come pure

" l'impressione generale che famo le altre parti, farebbero attribuire gli avanzi, se si

" debbano reramente considerare i denti come da latte, ad un giovane animale. Si

" potrebbe anche, dietro la conformazione dei frammenti del mascellare e delle ver-

* tebre e persino dei denti semplici, conici (fig. 4,5,6), ritenerli per quelli di un

" vero Delfino, se oltre ai denti conici provreduti di una sola radice (fig. 4, 7) non

" ve ne fossero altri appiattiti, più larghi, i quali offrono in vista due (fig. $1 a, c$ ) o

" tre (ebend. b) radici, mentre due denti (fig. 7,8) mostrano al loro bordo anteriore

" ed un terzo (fig. 9) come un quarto (fig. 2 a) mostrano tanto al lor bordo ante-

" riore quanto al posteriore dentini, la considerazione dei quali, come pure della forma

" generale dei denti, ci obbliga senza esitazione alcuna a ravricinarli agli Squalodon ed

" agli Zeuglodon. I denti ad una sola radice, provreduti di una corona conica (fig. 4,

" 6,7$)$, corrispondono infatti, ben senza dubbio, ai denti anteriori; quelli per lo più

" a due radici, larghi, appiattiti, or denticolati solo sul bordo anteriore (fig. 7,8 ) or

" anche sul bordo posteriore (fig. 2 a e 9), corrispondono invece ai denti mascellari

* degli Squalodonti. 
"Come dimostra l'ultimo dente mascellare $(a)$ in posto sul frammento di man" dibola disegnato fig. 2 , il quale è denticolato sia sul bordo anteriore che pure sul " posteriore, i denti denticolati su entrambi i bordi sono gli ultimi. In conseguenza lo

"Squalodon Gastaldii nella forma dei denti mascellari deve avere avuta la massima " analogia coi denti del cranio di Barie che Jourdan indica come specie a parte

" (Rhizoprion bariensis), che il Van Beneden però riferisce allo Syualolon Grate-

" loupii. Siccome però dello Squalodon Gastaldii non ci restano per lo più che denti

" isolati dei quali non si può dire qual posto abbiano tenuto nell'uno o nell'altro

* mascellare, siccome inoltre possono avere appartenuto ad un animale ancor giovane,

" cosi non si può per ora parlare con qualche sicurezza di alcuna identità o diffe-

" renza della dentizione dello Squalodon Gastaldii con quella dello S. Grateloupii

- o Bariensis.

"Lo S. Gastaldii si potrebbe, a quanto pare, distinguere dallo S. antverpiensis

" che nel disegno del Van Beneden arrebbe soltanto posseduti denti mascellari den-

" ticolati al solo bordo posteriore, per la presenza di alcuni o di parecchi denti ma-

" scellari denticolati ancora al bordo posteriore (1).

"Ancor di più che dalli Squalodon anterpiensis e Gratcloupii (bariensis?")

* lo Squalodon Gastaldii differirebbe dallo Squatodon Elratichii nella forma dei

" denti mascellari, poichè in quest'ultimo tutti i denti mascellari appaiono più larghi

« e denticolati tanto al bordo anteriore quanto al posteriore.

« Il dente mascellare disegnato fig. 9 si allontana del resto da tutti quelli che * io conosco, e che gli sono omologhi, degli altri Śqualodonti, in ciò che questo nel

" suo bordo anteriore lascia distinguere un solo denticolo (in vece di tre o quattro).

- $\mathbf{E}$ dubbio se questo stato di cose abbia a considerarsi siccome puro stato giovanile

- oppure a riferirsi a differenza specifica. Se la seconda interpretazione fosse la vera,

" essa darebbe in qualche modo un carattere per la distinzione dello Squalodon

" Gastaldii. Per la seconda interpretazione, che la forma del dente disegnato fig. 9,

" il quale infatti appartiene ad un piccolo animale, sia da considerarsi come un

" puro stato giovanile, non pare che parli in farore il fatto che sul frammento di

" mandibola disegnato fig. 2 si trova in posto un dente $(a)$, uno dei posteriori è

" vero, un po' piu grande, arrotondato, più largo, quadridenticolato sul inargine an-

" teriore, tridenticolato sul posteriore.

« Le due parti basali della mandibola (fig. 1 e 2), delle quali la figura 1 appar-

« tiene al ramo destro, la figura 2 al sinistro, corrispondono essenzialmente alle " omologhe parti dei Delfinidi.

"La sua pars ascendens si distingue però per la sua considerevole altezza, " principalmente per quella della sua parte posteriore. Il frammento rappresentato * fig. 3 appartiene alla metà o all'estremità della mandibola.

«Se si paragona il più completo dei frammenti di mandibola dello Squalodon « Gastaldii (fig. 1) colla parte sua corrispondente della mandibola appartenente senza « dubbio allo Squalodon Grateloupii, rappresentata nel modo il più preciso nella " tav. XXV da J. Müller (d. Zeuglodonten), se ne ricava che la mandibola dello

(1) Voleva forse dire: anche all'anteriore. 
"Squalodon Gastaldii appare, principalmente daranti, decisamente più alta e ne

" differisce sostanzialment?. Se si paragona il nominato frammento di mandibola dello

" Sq. Gastaldii con quello del cranio del Ihhizoprion baricnsis di Jourdan (Ann.

* d. Se. Nat., 4 e Sér., t. XVI, pl. 10), la mandibola dello Sq. Gustaldii apparisce

" al di dietro decisamente più bassa, allo avanti invece più alta. Sventuratamente

" non potè essere pure presa in comparazione lu mandibola dello Squalodon Antver-

"piensis nè dello Ehrlichii poichè di entrambi sono conosciuti soltanto tali fram-

" menti di mandibola da non permettere alcun confronto.

"Le indicate differenze dello $S q$. Gastaldii dovrebbero del resto accennare ad

- altre differenze nel cranio ed a far apparire fondata la distinzione specifica del mede-

- simo dal vero Sq. Grutcloupii H. v. MEr. e dall' ammessibile $S q$. bariensis. Qual

" carattere differenziale, invece, dallo Siqualodon Antverpiensis el Elwhichii non può

" finora essere indicato altro che quello l'ato più su del modo di comportarsi dei

" denti mascellari.

"Per il completo e sicuro stabilimento della specie Squalodon Gastaldii si

" dovrebbero bensi riportare ancora le differenze del medesimo dai dubbiosi Squu-

- lodon Scillae, Sucssii e Cutulli. Siccome però mancano tutti gli aranzi adatti ad

" un confronto, cosicchè per ora non si può ragionare nè in fivore di una, possibile

" in vero, identita dello Srmulodon Grustuldii con l'una o coll'altra delle dubbiose

" specie citate, trovate in Italia esse pure, nè per la sua difféenenza da esse, cosi gli

" ho aggiunto un (!)

"La vertebra cervicale disegnata fig. 10 e 10 a apparisce come delfinoide.

"Lo figure 11 e 12 mostrano una delle vertebre dorsali medie, con processo " spinoso alto, piuttosto stretto, che ricorda vertebre simili che si scorgono nei Delfini.

"Le figure $13,14,15$ mostrano una delle vertebre dorsali posteriori, a quanto

" pare, carenata al di sotto. La figurcu 16 rappresenta senza dubbio una delle vertebre

" lombari posteriori come lo dimostrano chiaramente la forma del suo corpo e quella

" del processo trasverso conservatoci, il quale ultimo ricorda, per la sua estremità

" allargata, diversi Delfinoidl, specialmente i Chumpsodelphiu che, avuto riguardo

" alla forma delle mascelle, specialmente delle mandibole, si accostano agli Squa-

* lodonti.

"Liesemplare rappresentato fig. 17, 18 mostra pure di essere una vertebra

* lombare, in vero molto guasta. Che le figure 19-22 sieno disegrni di due delle ver-

* tebre caudali della regione anteriore, non però le primissime e neanco le ultime,

" lo mostrano i clue rilievi paralleli clella loro faccia inferiore serventi all' articola-

"zione dei processi spinosi inferiori (fig. 19-22).

"La costa isolata rappresentata fig. 23 dimostra cliaramente che anche questo

"dubbioso Squalodon, come molti degli estinti Delfinoidi o Balenoidi del periodo

" miocenico, abbia possedute coste grosse e forti.

"In generale si può ammettere che le vertebre e coste conservate, come affatto

« particolarmente la mandibola, stavano in vicinissimo rapporto con quelle dei Del-

" finoidi. $\grave{\mathrm{E}}$ del resto molto a deplorarsi che tra questi avanzi manchino quelli, cotanto

" desiderati, delle estremità ».

Alla storia dello Squatorton Gastaldii poco mi resta da aggiungere. 
Il Gerrais, a pag. 440 della Ostéographie des Cétacés fatta in collaborazione col Prof. Van Beneden, non si esprime a proposito dello Squalodon Gastaldii che colla seguente frase: " Enfin M. Branit a plus recemment appelé Squalodon Ga" stuldii des restes analogues découverts dans la Mollasse d'Acqui ».

Il Brandt non ne fa piu cenno nelle sue Ergacnaungen zu den fossilen Cetaceen Furopa's.

Nel 1876 il Prof. A. De-Zigno descrivendo i bei resti di Squalodon Catulli scoperti nell'arenaria miocenica del Bellunese, avendo precelentemente enumerate in un riassunto cronologico anche le ossa di Squulorlon Gastaluii della Collezione Gastaldi e dopo avere con una magnifica tavola ed una esatta descrizione illustrati i resti che fanno oggetto della sua Memoria, compara gli stessi avanzi con quelli dello Sq. Gastuldii, esprimendosi (a pag. 12 della sua Memoria) colle seguenti parole: " Ora "paragonando la specie del Bellunese » (Squulodon Cutulli) « cogli altri resti di "Squalodonte trovati in Italia e con quelle specie clie più mostrano di avricinarsi " alla nostra, osserveremo che lo Squalodon Gustuddii del Piemonte ha i denti non « solo di gran lunga piủ piccoli, ma con una corona relativamente piủ bassa e piú " angusta e dentellata in modo irregolare ».

Nel 1881 il Capellini descrisse nuovi avanzi di Squalodonte trovati nella Mollassa marnosa miocenica a Jano nel Bolognese; questi, per essere in piccol numero ed in cattivo stato, non presentavano molte parti comparabili colle specie già conosciute di Squalodon. Basandosi sui pochi dati riconoscibili, il Capellini attribui dubitativamente tali residui alla specie Squalodon Gastaldii. Se, come tutto induce a creclere, la supposizione dell'esimio Professore è vera, noi verremmo così ad avere, della nostra specie, conoscenza, oltrechè delle parti già descritte dal Brandt, anche di parte del lato, specialmente del frontale, destro dell'interessantissimo apparato auditivo, e di una falange (cose tutte che finora non conosceramo) insieme ad alcune altre parti che già Acqui ci aveva fornite, siccome una vertebra dorsale, un dentino, frammenti di coste, ecc.

Ne è dunque lecito sperare che nuove fortunate scoperte ci mettano sulla strada per venire alla esatta conoscenza di una specie che finora non s'è ancor lasciata che travedere nell'Alta Italia, ma che pur deve aver avuto un certo numero di individui a rappresentarla in diverse localitì del nostro paese.

Lo Squalodon quaternavium poi, che fu ultimamente accennato dal Forsith Najor (1), presenta caratteri tali da non essere confondibile con alcuna delle specie precedentemente note e quindi neppure colla specie che a noi pel momento unicamente interessa: lo Squalodon Gastaldii BRDT.

(1) Squalodon quaternarium di Monte tignoso. Atti d. Soc. Tosc. d. Sc. Nat. (Proc. verb.), lam gina 227. Pisa 1881 . 


\section{Genere Champsodelphis Gert.}

\section{N. 49. Champsodelphis? italicus Portis.}

FIG. 97-99.

Da molti anni esistono nel nostro Museo (non essendo però mai state pubblicamente esposte) tre vertebre indeterminate, state trovate a Camino, presso CasalMonferrato, in un giacimento marno-argilloso appartenente od al Miocene superiore orl all'infimo Pliocene.

Esse vennero trovate in compagnia di un' Anodonta, abbastanza ben conservata, di un modello deformato di uno Spatango, di denti di Carcharodon e di Lamna.

Nel liberarle dalla tenace argilla dentro a cui ancora si trovavano avrolte, mi accorsi che esse arevano doruto appartenere ad un qualche Cetaceo e che mentre l'una doveva aver fatto parte della regione lombare, le due altre erano comprese nella candale.

Ho rappresentato fig. 976 e $99 \zeta$ la vertebra lombare; è molto malconcia, manca di tutti i processi e lo stesso corpo è ridotto quasi alla metà del volume primitivo. La sua lunghezza è di $75 \mathrm{~mm}$., l'altezza della sua faccia posteriore si può valutare a $50 \mathrm{~mm}$. , la larghezza della medesima faccia a $50 \mathrm{~mm}$.

Le due epifisi sono già fermamente saldate alla diafisi $e$, nemmeno nei punti di rottura, non vi ha piu traccia di una primitiva separazione. Le due faccie articolari sono convesse nella regione periferica per un raggio di $8 \mathrm{~mm}$. circa, piane al centro, ciò che ci dà una grande estensione di movimento delle vertebre l'una sullaltra. La vertebra porta ancor la traccia dei due pedicoli dell'arco neurale. Sono molto lunghi e strettissimi, si impiantano sui tre quarti anteriori del corpo e comprendono un canale neurale strettissimo nel quale, ad egual distanza rispettiva dalla faccia articolare anteriore e dalla posteriore, si osservano due fori nutritizi, essi pure allungati e stretti, approfondantisi nel corpo dell'osso. Al disotto si osserva che il corpo della vertebra era fortemente carenato per tutta la sua lunghezza. La carena doveva molto allargarsi nel terzo posteriore della vertebra, probabilmente per porgere attacco al primo osso a $r$, cio che ci fa supporre abbiamo qui dinanzi una ultima lombare od una prima caudale.

Il processo trasverso sinistro manca completamente; il destro è rotto presso l'origine; esso era impiantato sotto alla metà altezza del corpo e ad un dipresso ad egual distanza sia dalla faccia articolare anteriore che dalla posteriore, forse un po' più verso la prima; non era attraversato alla base da alcun canale arterioso ed erc molto allungato e relativamente solido; in tal guisa avrebbe potuto sopportare anche una forte espansione allo infuori.

Finalmente sulla superficie latero-superiore destra, ben conservata, una forte ruga accusa la linea di saldatura dello anello neurale col corpo: e rugosità dovute alla stessa origine si osservano tutto attorno alle superficie articolari seguendo le linee dove arrenne la saldatura tra le epifisi e la diafisi. 
In complesso questa vertebra ultima lombare o prima caudale (come gia dicemmo) fa l'effetto di aver appartenuto ad un Cetaceo affatto adulto e di essere, proporzionalmente alle altre dimensioni, molto allungata.

La seconda vertebra, pur figurata (fig. $97 a, 98,99 a$ ), è, quantunque incompleta ancli'essa, molto meglio conservata della prima. La sua lunghezza è di $45 \mathrm{~mm}$.; la larghezza e l'altezza della superficie articolare posteriore, eguali, misurano ciascuna $38 \mathrm{~mm}$. circa. Anche qui le epifisi sono gia completamente saldate alla diafisi ed ancora qui non si osserva nei punti di rottura alcuna interruzione fra le tre parti primitive dell'osso. Le faccie articolari sono, come per la vertebra precedente, lievemente convesse per la parte corrispontente alla periferia, piane od anche lievemente subconcave al centro, facendo nell'insieme l'effetto di faccie convesse. Lapofisi spinosa è rotta poco piu in alto della sua origine, è impiantata sui tre quarti anteriori del corpo della vertebra ed attraversata longitudinalmente ancora dal canale neurale, ridotto a non arer piu che $3 \mathrm{~mm}$. circa di altezza per 2 di larghezza. Questo canale, alla sua escita posteriore dalla volta dell'arco, si continua pel resto della lungezza del corpo della vertebra in un lieve solco: da questo partono, immediatamente dietro a ciascun pedicolo, anzi incidendoli leggermente, due grondaie, una a destra ed una a sinistra (quest'ultima piu marcata), incarate nella superficie dell'osso e discendenti obliquamente verso l'origine di ciascun processo trasverso il quale è poi traforato in corrispondenza di esse.

I processi trajversi non sono più gran che espansi allo esterno. Anteriormente, dore raggiungevano il massimo di divergenza dal corpo, dovevano arer la loro estremità-allo esterno di $12 \mathrm{~mm}$. dallo accennato forame arterioso.

La superficie inferiore di questá vertebra è altrettanto caratteristica della superiore. Nel mezzo essa era segnata da due rilievi longitudinali più larghi alle estremità anteriore e posteriore, per dare superficie articolari alle ossa a $V$ delle vertebre rispettivamente anteriore e posteriore, e più strette nel mezzo. Qui venivano attraversati ciascuno da un forame sboccante allo esterno in una grondaia che, partita dal forame arterioso attraversante il processo trasverso di ciascuna parte, si dirigera, circondando il corpo della vertebra, ma facendo nella superficie di esso una incisione profonda, contro tali rilievi ossei longitudinali, li traversava e raggiungeva così il canale emale. Posteriormente ciascuna di queste stesse grondaie, ma prima di raggiungere i detti rilievi, dava lapprima una ramificazione diretta obliquamente allo indietro ed in su, girante per conseguenza dietro il margine posteriore del processo trasverso : poi una obliqua allo indietro ed in giu che andara probabilmente a finire sulla faccia esterna del sottostante osso a $V$. Anche queste due ramificazioni sono, tanto a sinistra quanto a destra, altrettanto profondannente incise nella superficie del corpo cle la grondaia principale; hanno però un brevissimo percorso poichè la superiore non ha più di $5 \mathrm{~mm}$. di lunghezza, la inferiore 4. Finalmente, a render completa la descrizione di questa vertebra, ricorderò come ancora su di essa sieno marcate con due forti rugosità longitudinali le linee dell'antica saldatura dell'arco neurale col corpo della vertebra.

Tale vertebra fa l'effetto, in generale, di arer appartenuto ad un piccolo Cetaceo affatto adulto e di esserne stata una delle caudali posteriori, di arer aruta una lunghezza considerevole in confronto delle altre sue dimensioni e che abbia potuto fir parte dello stesso individuo cui appartenne la vertebra precedente: 
La terza vertebra trovata è in molto più cattivo stato delle due precedenti, inquantochè gran parte della sua superficie è perduta. Essa lıa una lunghezza di $52 \mathrm{~mm}$. per 32 di larghezza el altrettanti di altezza alla sua faccia articolare posteriore. Presenta, come la seconda vertebra descritta, i processi trasversi perforati alla base dal passaggio dell'arteria ed in seguito profonde grondaie che si continuano sotto ai rilievi di sostegno delle emapofisi, il canale neurale e l'apofisi neurale molto guasti e ridotti, e, segnate da rughe, le linee di saldatura dell'arco neurale e dei processi trasversi col corpo. Anche questa è una vertebra caudale e si distingue dalla precedente per essere piu lunga, meno alta e men larga, per maggiore sviluppo dei processi trasversi e minore sviluppo dell'arco neurale. Sarei per credere che essa appartenga alla stessa specie della vertebra caudale precedente ed abbia avuto avanti a sè press'a poco lo stesso numero di vertebre caudali di quella, ma che abbia forse appartenuto ad un altro individuo dove il carattere dell'allungamento delle vertebre era ancor più marcato.

Trovo che vertebre lombari e caudali affatto simili a quelle che tentai di descrivere, e presentanti gli stessi caratteri essenziali ed accessori, furono trovate nelle argille pliocenico-inferiori di Nussdorf presso Vienna e che distinte in due specie ricevettero dal Brandt i nomi di Champsodelphis Karreri e Fuchsii.

Le nostre vertebre di Camino si accostano di preferenza a quelle descritte sotto il nome di Ch. Fuchsii, come lo si può ben verificare confrontando le nostre figure con quelle date dal Brandt (op. cit., tar. XXIX, fig. $1 F, G, H$ ). Ne differiscono però per le dimensioni molto maggiori e per avere ancor piu marcato il carattere dello allungamento del corpo.

11 genere Chrmpsodelphis stabilito da P. Gervais è, come ognuno sa, un genre della famiglia dei Delfinorinchi, caratterizzato dalla simfisi della mandibola straordinariamente allungata, occupando almeno i due terzi della lunghezza della mandibola stessa (questa è inoltre molto appuntita e senza solchi longitudinali che ne dividano la faccia inferiore in più campi) da denti a corona breve, appuntita, rigonfia alla base, talora provreduta di un'appendice basale e con forte e grossa radice, da vertebre cervicali libere, da corpi delle vertebre lombari più o meno allungati, ma sempre piu lunghi che alti e forse da processi trasversi delle stesse lombari, mediocremente allungati el allargati all'estremità.

Molti di questi caratteri non ho potuto naturalmente verificare: se ho attribuite le vertebre di Camino al genere Campsodetphis, gli è perchè i caratteri che esse presentano non trovarono riscontro che in quelli di pezzi omologhi di altre specie attribuite al genere stesso; ho seguito in ciò l'esempio del Brandt, il quale pure attribui al gen. Champsodelphis vertebre isolate lombari e caudali trovate a Nussdorf, servendosi delle sole analogie che esse presentavano colle vertebre omologhe di altre specie meglio conosciute precedentemente trovate sia in Austria sia in Francia, e la cui determinazione e denominazione fu accettata dallo stesso Gervais che aveva primo proposto il genere.

Se la presenza del genere Champsodelphis si avvererà in Piemonte, un nuovo ed interessante tipo arricchirì la nostra fauna cetologica terziaria, già d'altronde molto ricca ed importante. 


\section{Genere SCHIZODELPHIS Gerv.}

\section{N. 50. Schizodelphis compressus PorTIS.}

Fig. 100-102.

\section{IL DELFINO FOSSILE DI BARBARESCO.}

Nel 1864, eseguendosi sutto Barbaresco presso Neive una galleria, nello spaccare una zolla di argilla vennero trovate in essa varie ossa assieme intrecciate, impastate, compresse. Le due parti della zolla furono raccolte e conservate dallo Ingegnere Petrini che ne fece dono al Museo di Mineralogia e Geologia dove furono conservate e le ossa riconosciute come aventi appartenuto ad un Delfino.

Occupandomi ad ordinare $\mathrm{i}$ nostri Cetecei fossili dovetti naturalmente tener conto ancora di questi avanzi, al quale scopo tentai di scoprire alcune ossa che non erano che in parte visibili. Quantunque tale lavoro non mi abbia dato tutto il risultato che avrei desiderato, tuttaria non fui malcontento di averlo eseguito, essendomi riuscito non solo di mettere allo scoperto varie vertebre, ma eziandio di raccogliere cinque dentini dell'animale, i quali isolati dalle mascelle e mandibole (perclute) giacevano qua e là nascosti per entro l'argilla. Colle parti precedentemente scoperte e con quelle da me poste a nudo, gli aranzi ora conosciuti di questo animale constano delle parti seguenti:

a) Cinque denti isolati quasi completi;

b) Impronte ed avanzi di almeno sette vertebre dorsali;

c) Impronte ed avanzi di almeno quattro epifisi di vertebre dorsali: due di esse oltre ad essere improntate dalla faccia diafisaria lo sono pure, nella controlastra, dalla faccia libera od articulare;

d) Avanzi di due o tre archi neurali, colle loro apofisi, isolati dai corpi di vertebra;

c) Avanzi più o meno importanti, e talor semplici impronte, di almeno una ventina di coste; fra le altre porzione della prima di destra;

f) Impronta di un omero;

g) Impronta di un cubito;

7) Impronta e controimpronta di un radio:

i) Impronta di un ossicino del corpo;

j) Impronta di una falange.

Ho fatto figurare nelle tavole (fig. 100 e 101) le due lastre quali presentemente si trovano e (fig. 102) i cinque dei denti trovati scavandone l'argilla.

Chiunque esamini le diverse parti conservate sulle due lastre non tarda a riconoscere aver esse dovuto appartenere ad un piccolo Delfino; se però le ossa le si esaminano comparativamente l'una all'altra, si rimane colpiti dalla grande esilita di ciascuno in proporzione delle dimensioni che deve aver avuto l'intiero animale. Ve-

13 A. Portis. 
diamo per esempio delle coste che misurano 32 centimetri di lunghezza (secondo la curva), e che per conseguenza ci obbligano ad ammettere per l'animale in carne una circonferenza di almeno 90 centimetri nella regione toracica, essere attaccate a vertebre dorsali piccolissime, le loro faccie articolari non misurando che $23 \mathrm{~mm}$. di diametro trastersale (in questa regione, il maggiore); rediamo come le coste stesse sieno, in proporzione della loro lunghezza, sottilissime, e come le ossa del braccio e dell'avambraccio dividano le stesse particolarita, essendo, in proporzione delle dimensioni che deve aver possedute l'animale, assai lunghe ed esili, come piccolissime devono essere state le falangi, a giudicarne da quell'unica ed incompleta che abbiamo sott' occhio.

Carattere proprio a questo Cetaceo si è ancora quello di avere l'omero piủ lungo di ciascuna delle ossa dell' avambraccio e, probabilmente, con una testa abbrstanza grossa e rigonfia e di avere avuto il corpo delle vertelore dorsali (di cui già notammo le dimensioni trasversali) lungo un 28 a $30 \mathrm{~mm}$. e con faccic articolari piane.

Sul numero delle vertebre della intera colonna, e sul numero di esse nelle diverse regioni, nulla di fisso è possibile dire, troppe essendo le parti mancanti dello scheletro. Cosi pure nulla possiamo dire sulla forma e rapporti del cranio mancandoci questo completamente.

Ia questo fossile che, nello stato in cui renne rimesso al MIuseo, non mostrava le parti necessarie per potere con sicurezza stabilire a quale famiglia di Delfinidi esso poteva renire ravriciuato, forni in seguito organi che maggiormente ci illuminarono sulle sue analogie di famiglia: roglio dire di quei cinque dentini trovati nello scavare l'argilla per meglio fur risaltare e scoprire alcune delle coste.

Tali denti sono (come si vede nella fig. 102, dove essi sono disegnati in grandezza naturale) relativamente piccoli, il piủ grande di tutti non misuranco in complesso che $13 \mathrm{~mm}$. di lunghezza ed il piì̀ piccolo soli 6 .

Qualunque sia la loro dimensione, essi si presentano tutti colla stessa forma e proporzioni caratteristiche. Ciascuno di essi è munito di una grossa radice conica uncinata all'estremiti inferiore e che fa molto più della meti dello intiero dente. La coroua invece è ricoperta di uno strato relativamente spessissimo di smalto nerastro, fortissimo e scmitrasparente: ha una forma conica fortemente schiacciata dai fianchi, ricurva e finiente in una punta esile ed arrotondata. Tn altre parole, ciascuna corona ¿ compressa dallo avanti allo indietro (supponendo il dente in posto nel suo alveolo). munita internamente ed esternamente di un. taglio arrotondato, è ricurva allo indentro della bocca ed acutissima.

Passando in rassegna gli Odontoceti viventi e fossili fin qui conosciuti, trovo che per la piccola dimensione e pel numero dei denti il nostro fossile dovrebbe essere collocato fra i veri Delfunini nella sezione distinta da un rostro sottile munito di denti minuti e fittissimi e che viene dal Gervais compresa coi generi Prollelphinus ed Eudelphinus. La forma dei denti nel Delfino di Barbaresco ci portercbbe invece a ravricinarlo al genere Schizodelphis GERv. del Miocene francese od al genere Champsodelphis; insomma ai Delfinorinchi.

Già il Gervais areva trovato a Poussan un dente presentante gli stessi caratteri di questi nostri ( il était grêle et sa couronne était bicarenée, presque à double «tranchant »), ma di dimensioni maggiori; egli l'aveva fatto figurare nella Zoologie 
et Paléontologie Françuise, $2^{\circ}$ édit., pl. 83, fig. 8, e l'aveva riferito al Delphinorhinchus sulcatus il di cui cranio venne rinvenuto in altra localita francese. Più tardi nella sua Ostéographie des Cétacés vivants et fossiles, parlando dello Schizodelphis (Delphinorinchus) sulcatus, ricorda quello stesso dente e dice: " un de cet organes " qui a été trouvé à Poussan et qui m'a alors paru devoir appartenir à la même " espèce était grêle et. sa couronne était bicarenée, presque à double tranchant. Je « doute aujourd'hui de cette assimilation ». Anche accogliendo tale dubbio del Gervais quanto all'identità specifica ed anche generica del dente di Poussan collo Schizodelphis sutcatus, è però sempre cosa evidente che i denti i quali, per i loro caratteri. più ricordano quello di Poussan e quelli di Barbaresco sono ancora quelli osservabili nella famiglia dei Delfurorinchi e particolarmente quelli dei generi Champsodelphis e Schizodelphis. Per conseguenza, quantunque io riconosca che sarebbe qui il caso di proporre per il nostro fossile un nuovo genere, motivandolo colle particolarità di forma, di posizione, di volume dei denti, stimo più prudente l'attendere a che nuovi e più completi aranzi vengano trovati, e soltanto da questi prender le mosse per una completa descrizione di questo genere intermediario a due famiglic di Delfinoidei oggidi perfettamente distinte, e per intanto inscriverlo come nuova specie sotto il nome di Schizodclphis compressus nel genere Schizodclphis, al quale presenta le maggiori affinità.

Lo Schizodelphis compressus nob. venne atunque, come dissi più in alto, trovato a Barbaresco presso Neive in un terreno argilloso appartenente, a quanto pare, al Hiocene superiore.

\section{TRIBUं DEI DELFININI.}

\section{Genere TURSiOPS Gray.}

\section{N. 51. Tursiops Cortesii (DESM.).}

\section{IL DELFINO FOSSILE DI MOMBERCELLT.}

Il Museo di 'Torino possiede (1) l'originale trovato nelle argille plioceniche di Mombercelli, descritto or fa un anno dal Capellíni o da lui stato determinato quale appartenente alla specie Tursiops Cortesii (DESM.). Non avendo nulla di nuovo da aggiungere, relativamente a questo cranio, rimando alla descrizione dello egregio autore inserta nel vol. III, serie IV delle Memorie dell'Accademia delle Scienze dello Istituto di Bologna (sessione 27 aprile 1882).

(1) Dirò meglio: si credeva di possedere tale originale, che infatti rimase più anni in Museo sempre reclanato dal proprietario, che si sperava si sarebbe infine indotto a venderlo. Tale speranza è stata sgraziatamento d3lusa: nè argomenti, nè offerte lo poterono decidere, e fú forza cedere finalmente ai suoi reclami. 


\section{N. 32. Tursiops Cortesii (DESM.).}

FiG. 103-105.

IL DELFINO FOSSILE DI CORTANDONE.

Oltre al suaccennato cranio del Tursiops Cortesii, il Museo Geologico di Torino possiede ancora un altro cranio nutilato, stato raccolto per cura del Professore Gastaldi a Cortandone presso Asti, nel 1862, e riunito or fa un anno alle Collezioni di Paleontologia del Mruseo stesso.

Molte delle ossa di questo cranio essendo molto più ben conservate e visibili che nell'esemplare precedente, ho creduto bene di darne una figura ed accompagnar quella con qualche cenno descrittivo. L'esemplare è dunque rappresentato (fig. 103) dalla faccia superiore, (fig. 105) di fianco, (fig. 104) dalla faccia inferiore. Esso è rotto alle sue estremità anteriore e posteriore e da quest'ultima parte noi vediamo l'interno della cavità craniana tutto tappezzato di sabbia indurita e solidissima. La lunghezza del frammento è di m. 0,435, la larghezza intera del cranio in corrispondenza dei limiti posteriori delle orbite di m. 0,280.

Della faccia superiore sono molto ben visibili e nettamente discernibili l'uno dall'altro i mascellari, gli intermascellari, l'etmoide, i nasali e l'occipitale superiore. 1 mascellari mancano della estremità anteriore, la parte conservata scorre lungo il rostro per una lunghezza di 20 centimetri mantenendo il loro bordo esterno quasi parallelo allo interno e la superficie leggermente inclinata allo infuori; posteriormente si espandono allo infuori per venir a formare l'arcata sopraorbitale raggiungendo il massimo di loro estensione in corrispondenza dell'angolo posteriore dell'arcata stessa. Da questo punto il loro margine esterno si arrotonda ripiegandosi gradatamènte indentro fino a diventare margine posteriore e dopo aver corso per un certo tratto lungo ed allo aranti della cresta occipitale, si ripiega allo avanti, allo infuori dei nasali, e va a perdersi nella linea di sutura longitudinale tra $\mathrm{i}$ mascellari e gli intermascellari.

Gli intermascellari, essi pure mancanti della estremità anteriore (una porzione che non doveva eccedere i $12 \mathrm{~cm}$. di lunghezza), hanno la loro faccia superiore leggermente attondata e piovente allo esterno. Conservano nel tratto corrispondente al rostro pressochè la stessa larghezza, divaricando leggermente fra loro all'origine del rostro stesso e più allo indietro incavandosi per fare il fondo della fossa nasale anteriore. Quindi la porzione posteriore del loro bordo interno è ampiamente intagliata dalla carità nasale, mentre il bordo esterno continua il suo andamento verso la cresta occipitale ripiegandosi con ampia curva prima allo esterno e poscia di nuoro allo interno fino a venire a confondersi col bordo postero-interno e terminare cosi l'osso con una punta che tocca il bordo esterno della lamina trasversale dello etmoide.

Tale lamina è per la maggior parte ancora coperta dalla roccia; se ne vede soltanto la estremità superiore quasi verticale e lievemente inclinata allo avanti.

I nasali sono due grossissimi tubercoli quasi affatto simmetrici collocati accosto alla linea mediana e formanti la parte culminante del cranio. 
La regione occipitale, quantunque in gran parte mancante, $\dot{e}$ tuttaria interessantissima. Noi vediamo come una grande squama ossea (che non lascia ben distinguere alcuna sutura) discenda quasi a rettifilo dalla cresta occipitale allo indietro e come tale squama ossea sia da tenersi come l'occipitale superiore. Quest'osso si è già espanso allo aranti ed allo insủ fino a venir a contatto coi frontali e quasi coi mascellari ed è doppiato profondamente da un'altra fortissima lamina ossea (che in alcuni punti è a sua volta doppiata da una terza), che dà origine, verso l'interno della cavità craniana, alla cresta di sostegno della falce cerebrale, e nella quale dobbiamo riconoscere $\mathrm{i}$ parietali e gli interparietali confusi e saldati insieme.

Sui fianchi e dal di sotto del cranio noi scorgiamo dapprima la porzione rimastaci dei mascellari superiori, la faccia inferiore dei quali è sensibilmente piana e che mostrano presso al bordo esterno: il sinistro, nove, il destro, dieci grossi alreoli nei quali erano impiantati i denti oggidi per sventura tutti mancanti.

Ai mascellari seguono allo indietro i palatini, abbastanza ben conservati, quindi parte dei pterigoidei, quella sorratutto che forma la cornice esterna delle cavità nasali posteriori (o meglio inferiori). Queste sono ancora presentemente ed affatto come nel fresco divise dalla lamina longitudinale etmoidea e limitate allo indietro dalla lamina trasversa dello stesso etmoide visibilmente seguita allo indietro dal presfenoide, il quale a sua volta è distinto per sutura dal corpo del basisfenoide.

Il basisfenoide poi ha un alisfenoide sinistro ancora magnificamente conservato ed in rapporto colle porzioni verticali del parietale e del frontale che formano la parete interna della fossa temporale. Manca sventuratamente tutta la porzione posteriore al parietale, cioè i temporali, la massima parte dell'occipitale e tutto l'apparato auditivo dalle due parti.

Nella volta poi della cavitì orbitale e della temporale osserviamo ancora la porzione rigonfia dell'osso giugale. Ne manca invece la parte stiloidea passante sotto l'occhio; mancano pure del loro bordo esterno i frontali, che sono ricoperti, come gia potemmo vedere, su tutta la loro superficie esterna dai mascellari.

Confrontato questo teschio con quello del Tursiops Cortesii (del quale esiste un modello in Collezione), ho potuto assicurarmi che,-malgrado il maggiore sviluppo delle ossa nasali, malgrado l'obliterazione della cresta occipitale, malgrado la maggioxe verticalità della squama occipitale e malgrado lievi differenze di sviluppo nelle ossa della faccia inferiore del cranio, e che sulla faccia superiore le ossa intermascellari sieno un po' meno salienti sul piano delle mascellari, questo esemplare non potrebbe assolutamente venir collocato in specie diversa da quello di Milano. Trattandosi poi, come ne è qui il caso, di un individuo adulto, le differenze ricordate potrebbero tutto al piủ servire per far ritenere il cranio di Cortandone come arente appartenuto ad un individuo femmina, mentre quello di Colle Torrazza conservato a Jilano potrebbe venir ritenuto come di individuo maschio.

Similmente potremmo ritenere per quello di un individuo femmina o di un giovane il cranio di Mombercelli descritto dal Capellini sul quale è osservabile maggior quantità di parti, ma quelle in generale molto più mal conservate e sul quale, per quanto è visibile, e le dimensioni sono ancora inferiori a quelle del teschio di Cortandone e le creste soprasuturali appaiono eziandio pochissimo sriluppate. 


\section{N. 33. Tursiops Cortesii (DEsM.).}

La Collezione del Museo di Geologia di Torino possedera già da lungo tempo alcuni denti trovati nelle sabbie gialle a Baldichieri, nell'Astigiana, ed aventi fatto parte, in altri tempi, della Collezione Sotteri. Lo stesso Abate Sotteri ne avera pur donati parecchi, stati trovati contemporaneamente, alla Collezione dell'allora Istituto tecnico, piu tardi R. Scuola di Applicazione per gli Ingegneri in Torino. Colla riunione del materiale raccolto dal Gastaldi a quello del Museo di Geologia, rennero anche ricuperati questi denti. Veniamo ad averne ora diciannove in tutto. Tali denti, sia col gruppo conservato al Museo, sia con quello conservato al Valentino, vennero presi in considerazione da varii autori. Il Falconer opinò che fossero di Coccodrillo; il Sismonda E. li attribui a Delfino (Cetaceo affine ai Cachalots) e ne areva fatti disegnare sei, di cui uno sezionato, nelle figure 9-14 della tavola (ora inedita) che egli areva fatto preparare onde unire al suo studio sui Cetacei piemontesi.

Il Gastaldi, che ritenera pure questi denti come di Delfino, ne fece pure disegnare tre nel 1874 e mandonne $i$ disegni al Brandt a Pietroburgo. Questi nella tav. V, fig. $15 a, b, c$, delle sue Ergacnzungen zu den fossilen Cetaceen Europa's ne diede la figura, ed a pagina 49 dello stesso lavoro cosi si esprimeva a loro riguardo: «I seguenti partecipatimi disegni in grandezza naturale (fig. $15 a, b, c$ ), che devo « pure al signor Prof. Gastaldi, somigliano bensi a quelli che il Gervais (Zool. et

"Patcont. Frans., pl. XX, fig. 5, 6) fece disegnare ed attribui al una Phoca, come "pure al dente da lui descritto siccome quello di una Phoque de Poussan (Ibid., " pl. XXXVIII, fig. 8). Siccome però, a quanto mi riferisce il Gastaldi, di questi "denti se ne son trovati parecchi nel circondario di Asti, cosi non si potrebbe leg"germente ammettere che si fossero trovate appunto soltanto le difese di una Foca. «Si trova quindi piu naturale il supporre che essi abbiano appartenuto ad un Ce« taceo dentigero. Aruto riguardo alla loro estremità superiore, essi somigliano benși " ancora ai denti anteriori dello Zeuglodon (1Tüll. Zeuglod., Taf. XXI, fig. 3-5) « e dello Squaloclon, ma la parte inferiore, non aperta, della radice non dà nep" pure appoggio a questa interpretazione. In riguardo alla loro forma e grandezza « paionmi ancora rassomigliare sovratutto a quelli del Globiccphalus globiceps di « media età. lissi potrebbero tuttavia appartenere ad alcuno dei Delfinatteri italiani « descritti.

«Nel Museo di Torino, come pure nella Collezione Gastaldi, se ne trovano «parecchi».

È noto come il Brandt considerasse il genere Delphinapterus di Lacépède sinonimo del genere Bèluga di Gray e che comprendesse in questo genere il Delphinus Cortesii Dessr. ed il Delphinus Brocchii Bals: Criv:, stati più tardi riferiti al genere Tursiops. Per conseguenza il Brandt colle ultime delle parole qui sopra citate allude appunto alla pertinenza possibile dei denti in questione al Tursiops Cortesii od al Tursiops Brocchii. 
Ho confrontato ciascún dente col modello del Tursiops Cortesii esistente a Milano e colle figure dello stesso teschio e degli avanzi del Tursiops Brocchii ed ho potuto convincermi come il Brandt, partendo dal solo esame dei disegni, si sia accostato alla verita. I denti infatti, sia per la forma che per le dinensioni loro, non sono in alcun modo discernibili da quelli del Tursiops Cortesii. Ancora il numero loro (13) farebbe supporre appunto che noi aressimo la dentizione delle sole mandibole o delle sole mascelle, delle quali ancora alcuni denti sieno andati perduti.

Ora, nell'Italia superiore (nei terreni pliocenici) noi non abbiamo ancora del genere Tursiops che due specie stabilite: il Tursiops Cortesii ed il Tursiops Brocchii, Del prino noi abbiamo già dinanzi, provenienti dall'Astigiana, due teschii, ed il secondo non tarderà, secondo il Gervais el il Capellini, a non essere piu tenuto che come semplice varieta del primo. Trovo quindi naturale di considerare i diciannove denti di Delfino della Collezione Sotteri come aventi appartenuto all'unica specie risultante, al Tursiops, Cortesii. (Desar.).

\section{N. 34. Tursiops Cortesii (DFss).}

Il Museo Geologico 'I'orinese possicde, grazie sempre alla Collezione Gastaldi, alla quale provennero dalla Collezione Sotteri, sei vertebro le. quali si manifestano decisamente delfinoidi. Esse appartengono ad un individuo gi̊̀ quasi adulto; infatti le epifisi sono, già sufficientemente ben saldate alla diafisi, quantunque due delle vertebre manchino della epifisi anteriore e della posteriore ed una della anteriore.

In queste vertebre tutto l'arco nsurale è ancora molto ben spiluppato ed il canale è altissimo e molto ristretto. 'Tanto il processo spinoso che i trasversi sono mutilati. Delle sei vertebre (fra loro consecutive) le due anteriori mostrano la facciu inferiore percorsa longitudinalmente da una sola carena che però si stoppia presso al margine posteriore nella seconda di esse: le altre sono nella stessa località percorse da due rilievi paralleli ingrossati presso al margine posteriore per dare attacco allo ossa a $V$ ossia all'arco inferiore. Abbiamo per conseguenza clinanzi una vertebra appartenente ancora alla regione lombo-sacrale e le cinque prime caudali, considerando come prima caudale quella che precede il primo osso a $V$.

È noto quanto bisogni andar cauti nel dare nomi specifici a simili ossa, molto rassomigliandosi fra loro le vertebre delle regioni caudali delle diverse sezioni di Delfinidi. Aruto però riguardo che quelle che mi stanno davanti concordano esattamente nella forma e disposizione delle parti con quelle della stessa regione del Tursiops Cortesii, che esse ne son lievemente più piccole, il che tanto piu si spiega per essere l'individuo cui esse appartennero un po' più giovane dell'individuo tipo di Milano, ed avuto riguardo finalmente che il Tursiops Cortesii visse e lasciò non tropp̃o scarsi inclizi della sua presenza nel nostro Pliocene, ho attribuito alla stessa specic ancora le sei vertebre che ho dinanzi.

Noterò ancora incidentalmente come l'individuo cui queste vértébre appartennero servi probabilmente di alimento a Squali o ad altri pesci di non gigantesche dimensioni, i quali lasciarono sopra il corpo di due di tali vertebre traccie dei loro aguzzi 
denti, sotto forma di intagli obliqui rivolti in vario senso e che qualcuno potrebbe interpretare come doruti alla mano dell uomo.

Queste sei vertebre sono state trovate nell'Astigiana in località non ben precisata (1).

\section{N. 3้. Tursiops n. sp. (miocaenus).}

Fig. 106.

\section{IL DELFINO DI ROSIGNANO.}

Per chiudere la serie dei Delfini fossili piemontesi spettanti al genere Tursiops, non mi rimane più che a segnalare gli avanzi trovati nel Miocene medio a Rosignano dal sig. D. Bonelli, parroco di quel luogo, e da lui regalati al Gastaldi, col materiale del quale sono poi venuti in questo Museo Geologico.

Il tutto consiste in sei denti ancor relativamente giovani, per quanto giudicar si possa dalla natura della radice, e piuttosto maltrattati e rotti.

La forma delle corone, la natura del loro smalto, il modo loro di consumazione per l'uso, la parte che esse prendono sull'insieme del dente, la forma degli interi denti e le loro dimensioni me li fanno collocare affatto accanto a quelli precedentemente accennati del Tursiops Cortesii, dai quali non differiscono che per il grado di acutezza della corona, un po' più debole nei denti del T. Cortesii, un po' più svolta in questi.

Segnaliamo adunque come il genere Tursiops oltre ad aver rappresentanti viventi, oltre ad averne di fossili pliocenici, ne abbia ancora nel Miocene medio. I denti in questione non sono ancora tali pezzi da meritare un nome specifico, non arendo caratteri abbastanza salienti da farli distinguere dalle specie conosciute. Ci limiteremo per ora, e tanto per poterli nominare in discorsi arvenire, ad attribuirli provrisoriamente ad una specie ancor da trovarsi, il Tursiops miocaemus.

\section{Genere STENo Gray.}

\section{N. ə̋(. Steno Gastaldii J. F. BrandT.}

\section{IL DELFINO FOSSILE DI CA-LUNGA.}

Dorendo questo mio essere, più che altro, un Catalogo dei resti fossili di Cetacei rinvenuti nel Piemonte e nella Liguria, stimo, come già dianzi accennai, non inutile riportare testualmente, traducendoli ove ne sia il caso, i passaggi di opere, non sempre

(1) $N^{\circ} 51$ bis. Tursiops Cortesii (DESs1.). Nella state del 1884 vennero portate a questo Museo quattro vertebre caudali, regione anterioje, di un piccolo Cetaceo appartenente ai Delfinidi. Tali vertebre vennero trovate a Penango (mandamento di Tonco, circondario di Casale Monferrato) in uno scavo per una cantina. Esse mancano delle epifisi e di tutti i processi rotti presso l'origine loro. Esse paiono concordare assai bene colle corrispondenti ossa del Tursiops Corlesii, meno le dimensioni alquanto minori. Suppongo abbiano appartenuto ad un giovane individuo di questa specie, la quale, se la determinazione si potesse fare con sicurezza, verrebbe così ad acquistare una maggiore antichità (Nota aggiunta durante la stampa). 
a nostra portata di mano, nei quali vieno più particolarmente descritta qualcuna delle nostre specic.

Gli è perciò che, nulla essendo stato aggiunto per nuove scoperte alla conoscenza dello Steno Guastaldii, credo essere dover mio il dare la descrizione che ne faceva il Brandt nelle sue Ergaenzungen zu den fossilen Cetaccen Europa's, già più volte menzionate, a pag. 15, se non di ripeterne lo figure. Esssa suona: "Nell'anı 1869 « vennero scoperti nel circondario, appartenente al Piemonte, di Asti, presso Ca-lunga, " negli strati inferiori delle argille plioceniche di coli, i.resti di un Delfinino, i quali « il Prof. Gastaldi fece disegnare nel MIuseo di Torino dal sig. Rapetti onde gentilmente " comunicarmeli per il presento complemento.

"I disegni dati nella tavola II offrono diverse vedute di un assai considere« vole frammento del cranio o di vliverse parti del medesimo, specialmente delle parti " auditive interne e della scatola cerebrale del cranio; inoltre figure dell'atlante e "dello epistrofeo, di una vertebra lombare e di una costa (Vedi più in là la spie" gazione delle tavole).

« La forma del muso, i numerosi, conici, appuntiti denti, la simfisi della mun" dibola fatta di due parti distinte, come pure la vertebra lombare corta, provreduta « di processi trasversi non allargati alla loro estremita, avvicinano certamente l'animale « a cui questi resti appartemero al genere Delphinus. Siccome però i distinti rami " della mandibola posseggono una simfisi lunga superante un quarto della lunghezza " delle mandibole stesse, la fauce tuttavia (almeno da quanto si puó vedere dalla « bella figura 3) non offre alcun solco laterale allungato come pel Ditphinus, cosi * il Dellinide di Gastalli si può piuttosto considerare siccome mo Steno. La man« canza visibile alla vertebria lombare (per corto per separazione e per totale perdita « posteriore (1) sopravvenuta) (lelle epifisi ci indica del resto che gli avanzi appar« temnero ad un giovane individuo.

« Siccome poi gli stessi vennero trovati in argilla pliocenica, cioè il loro de* posito soprarrenne in un tempo abbastanza vicino, cosi è naturale il porre la " questione se essi non possano appartenere all'uno o all'altro genere o specie di "Delfinidi, non spettanti al genere Delphimus GraY, ancora viventi ed osservati finora " nei mari di Europa.

"Lo Steno Gastuldii differisce dal Tursio truncatus Gr.1Y = Detphimus tursio "per il muso piu lungo, piu stretto alla base ed in mezzo, piu laigo all'estremiti; « per gli intermascellari piủ larghi, più ravricinati l'uno all'altro e per la molto piủ " lunga simfisi della mandibola.

« Per quanto si può ricavare dalla descrizione di Gervais sul suo Delphimus Tethyos "(Zool. et Paléont. Frans., $2^{e}$ édit., pag. 303), gli somiglia bensì lo Steno Gastatdii " per la mancanza di solchi laterali delle fauci; se ne distingue però per la parte « spettante al muso, molto più lunga e larga. Del resto non è riferito se il Delphimus "Tethyos possegga una cortissima simfisi mandibolare e quindi appartenga forse al "genere Tursio, oppure ne abbia una lunghissima e sia quindi uno Steno.

(1) Le epifisi non sono perdute; ne abbiamo allo incontro un numero considerevole di staccate; solamente il Gastaldi non credette necessario il farle disegnaro cosi isolate come esse sono. D'onile la credenza nel Brandt non se ne avessero più traccie.

14 A. PORTis. 
«Dal Lagenorhynchus albirostris (GRaY, Synops. of Whales, p. 8, pl. XI) " differisce lo Steno Gastaldii per la lunga simfisi della mandibola come pure per un " muso molto più lungo e stretto.

"Il cranio del Delphinus rostratus di Cuvier (Ann. d. MLus., XIX, 9; Rech. «s. T. oss. foss., éd. 8", pl. 222, fig. 7,8), dal Gervais (loc. cit., pag. 301) consi"derato come tipo del suo genere Gliphidelphis, prima dal Gray (Catal. of mammal.,

"Part. I, Cetacea, London, 1850 , p. 131) riferito allo Stcno (per quanto a me " pare, evidentemente riferibile a quest'ultimo genere), se viene confrontato collo Steno

" Gastaldii mostra nella sua metà anteriore un muso molto più sottile, piủ aguzzo

" e più compresso specialmente nella sua parte anteriore, intermascellari molto piủ " stretti, ed una più lunga simfisi della mandibola.

"Lo Steno Gastaldii non si lascia dunque unire ad alcuno dei Inclfinini finora « osservati nei mari che bagnano l'Europa.

"Dalle specie extra-europee citate dal Gray (Synops. of Wales, pagina 5) " differisce lo Steno Gustuldii in generale per la sua parte mascellare allungata, debol"mente compressa e i suoi intermascellari allargati. Pare che esso si accosti in gene" rale di più allo Stcno attenuatus (Grax, Synops., ecc., pag. 5, pl. 28) e com"pressus (ib., pl, 27). Ma anche da questi differisce per la parte mascellare piủ

" larga, tanto nel mezzo che allo avanti (od almeno allo avanti), come per la mag" gior larghezza degli intermascellari.

"Tra i membri ancor viventi, extra-europei, del genere Steno non si trova « adunque neppure alcuna forma alla quale si possa riferire lo Steno Gastaldii.

"P'er ciò che riguarda gli avanzi descritti o ricordati quali di Delfinini nei "Fossilen Cetaceen, pag. 244 e seg., non si possono quelli, in parte per la loro " incompletezza, in parte pel loro scarso numero, tirare in confronto. Una mandibola " figurata dal Gervais (Ostéogr. d. Cétuc., pl. LVII, fig. 7, 7a) potrebbe intanto, " dietro la sua forma e specialmente in considerazione della sua lunga simfisi (nón ben " confacientesi, a quel che mi pare, ad uno Schizodelphis a Champsodelphis), essere " quella di uno Steno. Però la sua simfisi apparentemente più lunga ed i suoi rami più

" fortemente divergenti ed accennanti ad un muso piủ largo non ci lasciano pensare che

" essa sia per appartenere ad una predescritta specie alla quale debba venir unito lo

"Steno Gastaldii. Quest'ultima specie, dedicata in segno di riconoscenza al mio sti-

" matissimo amico di Torino, dovrebbe per conseguenza, anche in riguardo degli avanzi

" fossili a me fin qui noti, riguardarsi come ben fondata su caratteri craniologici.

«Il frammento di cranio, molto guasto, rappresentato dalla fig. 4, appartenente

" esso pure allo Steno Gastaldii, permette sventuratamente soltanto di riconoscere il

" carattere di Delfinile. Lo stesso dicasi delle parti dell'apparato uditivo (fig. 5, 6, 7),

" nei quali la Bulle non risalta distintamente.

"L'epistrofeo rappresentato in relazione coll'atlante nelle figure 8, 9 si accosta

" ad una simile riunione nei Delfini.

« È stato già più sopra notato che la vertebra lombare delle fig. 10 ed 11 è « fatta secondo il tipo delle vertebre del genere Delphimus in stretto senso.

«Sulla costa isolata, della quále la figura 12 ci offre un disegno, non ho pure * niente di speciale da ricordare. 
"Per riguardo alla grandezza lo Steno Gastaldii deve arer superato, a quanto «pare, il Delphinus detphis.

«In generale, dei membri del genere Steno sono a considerarsi come tali quelli " che dai Delfinini si accostano, per la lunga simfisi della loro mandicola, ai Plata" nistini; che però stanno più vicini, non a questi, ma ai primi, ai quali per conse« guenza derono stare uniti.

"Pare del resto interessante che lo Steno Gastalelii rappresenti un tal tipo « da procurarci un nuovo punto d'appoggio nella teoria che la fauna delfinoidca " primitiva (per quanto essa è conosciuta dagli avanzi del Terziario) abbia avuto un * carattere, se non affatto identico, certamente molto simile a quella ancor oggi virente, « e ciò per mezzo di numerose specie succedutesi ».

Alla bella descrizione del Brandt non aggiungerò che poche parole:

In primo luogo il Brandt non insiste sufficientemente sul carattere che presenta l'atlante dello Steno Gastaldii, di essere cioè completamente libero. Infatti, come se ne verle la linea di divisione dallo epistrofeo nella sua stessa figura, l'atlante forma un osso separato dallo epistrofeo. ì bensi vero che dallo strettissimo adattamento esistente fra le reciproche faccie articolari di queste due vertebre successive si può arguire che non potesse esistervi alcuna sorta di movimento dell'una sull'altra vertebra e che quincli per la comoditì dello animale tanto vale che esse siano staccate nella guisa della presente specie od unite come nelle altre specie di Steno. Na per il zoologo ed il paleontologo, costretti a tirar partito da questi caratteri, tale inclividualizzazione ha tanto più di valore in quanto si sa che nei Cetacei in generale le fusioni tra diverse ossa (le vertebre in particolare) non si acquistano coll'avanzare degli individui in età, ma preesistono cartilaginee nello stato fetale e che d'altronde è uno dei caratteri generalmente arnmessi al genere Steno-di aver le dette due vertebre insieme saldate.

Mi permetterò adunque di aggiungere alle caratteristiche già segnalate dal Brandt anche quella che il suo atlante ed il suo epistrofeo costituiscono due ossa distinte: e per dimostrare l'importanza di questo carattere me ne arrò a valere immediatamente descrivendo una seconda specie di Steno stata scoperta posteriormente in Piemonte.

In secondo luogo, occorre che io aggiunga come oltre alla vertebra lombare ed alla costa di cui parla e che figura il Brandt, esistono in Museo, spettanti alla stessa specie ed allo stesso individuo, numerosissime altre parti, siccome:

a) Molti frammenti delle ossa della faccia che sventuratamente non possono più aggiungersi alle parti disegnate dal Brandt (Gastaldi) per mancanza di frammenti intermediarii ;

b) Una ventina di denti isolati;

c) Quaranta vertebre, fra cui: le sei successive allo epistrofeo, poche dorsali, quasi tutte le lombari e molte caudali. Tutte queste vertebre, meno poche caudali, mancano di epifisi; tutte sono ridotte ai soli corpi, mancando gli archi ed i processi, e presentano tutte lo stesso evidente carattere di avere ciascuna la lunghezza del corpo sempre minore delle altre due dimensioni;

d) Una quantitì di processi trasversi isolati e rotti, tutti presentanti il carattere già segnalato dal Brandt, di non allargarsi alla estremità in alcuna regione;

c) Numerosissimi archi neurali isolati e rotti, tutti marcatamente delfinodei; 
f) Un 25 epifisi e frammenti di epifisi vertebrali, tutte dello spessore di circa un millimetro e mezzo, porcellanoidee, che più non trovano la vertebra cui appartennero; oltre a numerosissimi frantumi minori di altre epifisi vertebrali appartenenti alle diverse regioni;

g) Un 27 frammenti più o meno importanti di coste appartenenti ai due lati e rappresentanti la quasi totalità di queste ossa lunghissime, prismatiche ed esilissime.

Dal complesso di tutte queste ossa veniamo ad arguire ad un animale, il cui scheletro, benchè formato di numerose ossa, essendo queste corte, era pure relativamente assai corto; la regione toracica areva una sezione trasversale, il cui diametro supero-inferiore era grandemente maggiore del trastersale; la regione lombare relativamente lunga e la caudale anch'essa più alta che larga; insomma un animale molto elastico e sveltissimo nuotatore. Continuano però sempre a mancarci le estremità.

Di questo esemplare di Steno Gastaldii dovetti parlare in altro mio lavoro, presentando esso su alcune delle sue coste ed alcuni dei suoi processi spinosi e trasversi evidenti tagli e traccie di essere caduto rittima delle armi naturali dei Pesci-cani.

\section{N. ว7. Steno Bellardii Portis.}

$$
\text { Fis. 10i-1:?1. }
$$

11. DELFINU FOSSILE DI BAGNASCO.

Nel $1876^{\circ}$ il sig. Bignami, allora Allievo Ingegnere alla Scuola del Valentino, portara al Prof. Gastaldi l'estremità del muso di un Delfino fossile che egli avera staccato da una ripa nel territorio di Bagnasco, circondario di Asti.

11 Prof. Gastaldi inviava tosto il sig. L. Bottan, suo preparatore, a vedere se fosse possibile trovarne altri avanzi e, trovatili, estrarli. Se ne scopersero ancora numerose parti dello scheletro, che portate dal sig. Bottan a 'Torino, furono dal Prof. Gastaldi, per quanto possibile, ristaurate con quella pazienza e maestria che lo distinguevano, e da lui riconosciute come appartenenti a un Delfino fossile simile a quello che il sig. Brandt descrisse e figurò col nome di Steno, e restarono nella sua Collezione sin quando questa renne a fondersi con quelle del li. IIuseo Geologico.

Le parti dello scheletro di questo individuo presentemente conservate sono:

a) Il cranio mancante di parte delle pareti della scatola cerebrale, riempita dalla sabbia cementata, e di porzione considerevole anteriore del rostro;

b) I due rami della mandibola, gnasti allo indietro, mancanti della porzione anteriore e per lo più senza denti;

c) L'atlante e l'epistrofeo assai ben conservati;

d) Diciasette vertebre fra dorsali e lombari, di cui alcune complete, la maggior parte mancanti or dellarco neurale, or dei processi trasversi;

e) Sei o sette archi neurali di vertebre dorsali e lombari, isolati e rotti;

f) Quasi tutte le coste, rappresentate or da pezzi quasi completi, or da frammenti più o meno considerevoli; 
g) Numerosi frammenti di ossa che non poterono trovar posto preciso nello scheletro attesa la loro piccolezza e conseguente mancanza di caratteri.

L'esame delle ossa dello scheletro e del cranio ci dimostra come noi abbiamo dinanzi agli occhi un individuo già affatto adulto. Difatti tutte le ossa del cranio hanno già acquistato tra loro solicle relazioni e le suture si sono già ristrette e quasi cancellate, tutti i corpi delle vertebre sono gia completamente uniti alle cpifisi e non rimane piủ alcuna traccia della primitiva separazione; nessuna epifisi isolata, appartenente ad alcuna regione del corpo è stata trovata.

La parte conservata del cranio misura dallo indietro in avanti centimetri 44 . Però, come dissi, manca allo avanti una porzione considerevole del rostro. I'er giungere a determinare la lunghezza approssimativa dello intiero teschio lo comparato il teschio stesso con quello dello Steno plumbeus a cui il fossile presenta nelle parti conservate grandissima analogi:, e con due o tre dirersi metodi di misura sono giunto sempre pressochè agli stessi risultati.

Misuranclo nello Steno plumbeus prima l'intiero cranio dal margine posteriore dei condili occipitali al margine anteriore degli intermascellari e poscia la parte compresa tra un piano verticale toccante il margine posteriore dei condili occipitali ed un secondo passante pel margine anteriore delle ossa iugali, si vede come la seconda lunghezza eguagli i sette diciottesimi della prima: applicando lo stesso metodo alla ricerca della lunghezza del cranio di Steno Bellardii, troviamo che la lunghezza della parte espansa è di cm. 23; dividendo questo numero per 7 e moltiplicandolo per 18 reniamo a trovare per l'intiero teschio una lunghezza di oltre 59 centimetri.

Esaminando i rami della mandibola nella parte affetta ai denti, troviamo che in media un tratto qualunque di quella misura tanti centimetri di lunghezza quanti sono gli alveoli che essa porta. Ora noi abbiamo del ramo destro un frammento. che misura 36 centimetri di lunghezza: la porzione che gli manca per essere completato posteriormente e giungere così al margine posteriore del condilo articolare è di circa 2 centimetri di lunghezza; pel frammento in questione teniamo cosi $38 \mathrm{~cm}$. Del ramo sinistro abbiamo due frammenti: uno posteriore che misura dal condilo articolare allo aranti 35 centimetri, ed uno anteriore di 4 centimetri di lunghezza. Questo secondo non può adattarsi al primo, mancando un pezzo intermedio che, dalla comparazione dei due rami, non può essere inferiore alla eccelenza di lunghezza fra il frammento destro ed il sinistro; dobbiamo adunque collocare il nostro frammento di quattro contimetri alla distanza di tre centimetri dal limite anteriore di rottura del ramo sinistro ed in linea a quello; otteniarno cosi $42 \mathrm{~cm}$. Ma rispettivamente il secondo e piccolo frammento mostra che esso è stato rotto anteriormente proprio al limite posteriore della simfisi fra $\mathrm{i}$ due rami; ora noi sappiamo come sia caratteristico del genere Steno l'avere questa simfisi allungatissima; nello Steno sincnsis ogni ramo della mandibola porta, per la lunghezza tenuta dalla simfisi, non meno di 14 denti; Ia stessa parte nello Steno plumbeus ne porta da dodici a tredici e nello Steno rostratus, che ha i denti più rari, non ne porta che 12. Nel giovane esemplare di Steno Gastaldii che ho dinanzi e dove la simfisi non ha ancora raggiunta allo indietro tutta la estensione possibile noi troviamo, sempre nella stessa porzione, da nove a dieci denti ner ramo. Se per la man iibola dello Stcno Bellardii noi vogliamo pren?ere 
soltanto (in considerazione della loro reciproca distanza) quest' ultimo numero di dieci denti, se ammettiamo che questi organi siano nella regione anteriore della mandibola un po' più rarricinati e piccoli che nella posteriore e che quindi dieci denti possano aver stanza in una lunghezza di otto centimetri di ramo, agginngendo questi alla lunghezza precedentemente trovata pel ramo sinistro, arremo per tutto il ramo almeno 50 centimetri. Collocando ora la mandibola presso al cranio nella posizione che le tocca, noi vediamo che il cranio misura ancora dieci centimetri di lunghezza tra due piani verticali passanti, il primo, pei margini posteriori dei condili occipitali, il secondo, per le faccie articolari per la mandibola. Ne risulta alunque per l'intero cranio una lunghezza totale di 60 centimetri, cioè di meno di un centimetro superiore a quanto arevo calcolato col primo metodo.

Un terzo metodo che mi conduceva pressochè allo stesso risultato si fondava sulla osservazione che il rostro del genere Steno presenta, pressochè a metà della propria lunghezza, un lieve restringimento delle ossa mascellari, seguito, anteriormente, da lieve riallargamento, e come nell'individuo di Bagnasco il piano di rottura coincidera appunto colla località di restringimento del rostro, per cui, supponendo di aggiungere una lunghezza quasi eguale a quella della porzione conservata di rostro, si veniva appunto ad ottenere pel cranio intiero una lunghezza di 60 centimetri circa.

Ammessa dunque pel cranio una lunghezza di 60 centimetri, passiamo ad esaminarne le altre particolarità. Esso misura trasversalmente dall'un processo discendente del frontale o limite posteriore dell'orbita all'altro 26 centimetri. Nella sua faccia superiore osservansi molto bene i mascellari superiori, gli intermascellari, la porzione superiore della lamina etmoidea, le due piccole fossette da cui caddero i non simmetrici nasali, le ossa frontali, i parietali, l'occipitale superiore ed i condili occipitali. Di fianco, oltre le suddette ossa, scorgiamo: i giugali, gli squamosi, la regione sfenoidea e palatina e l'apparato uditivo (delfinoideo) sinistro; di sotto: le ossa della base del cranio solite ad osservarsi nella base del cranio dei Delfinini.

ben a ragione areva il Gastaldi considerato questo fossile come una specie di Steno, ed infatti, allorchè lo compariamo colle diverse specie viventi ben definite di questo genere, rediamo come con esse completamente concordi, sia nella forma del cranio, sia nella disposizione delle singole ossa, sia nelle proporzioni e sviluppo del rostro.

Crranio e mandibole (fig. 107, 108, 109, 110,111, 112, 113). - Se però noỉ cerchiamo di collocarlo in qualcuna delle suddette specie ci è impossibile di trovare quella che offra assolutamente gli stessi caratteri e che conseguentemente lo possa ricerere.

Cosi, quantunque lo stato dell unico cranio conosciuto dello Steno Gastaldii sia molto difettoso e le parti in esso conservate non siano sempre comparabili con quelle dello St. Bellardii, tuttavia noi non tardiamo a scorgere come il rostro dello St. Bellardii abbia dovuto esser notevolmente più allungato di quello dello St. Gúcastaldii ed abbia doruto portare maggior numero di denti, essendo essi circa 25026 per ramo di mandibola in questo secondo e 30 nel primo.

Oltracciò nello St. Gastaldii la faccia supero-laterale del rostro è pianeggiante, essendo più larghi e meno convessi gli intermascellari, mentre nello St. Bellardii la faccia indicata è molto più convessa e gli intermascellari più stretti e più elevati 
rerticalmente si sovrappongono di più ai mascellari. La sezione quindi del rostro sari nello St. Gastaldii proporzionalmente più larga e meno alta che non nello St. Belfardii, nel quale anche le due branche della mandibola sono conseguentemente piu ravricinate e la simfisi più lunga. In una parola, lo St. Gastaldii si avricina di più al tipo dello St. rostratus (GTiphidelphis GERv.), lo St. Bellardii a quello dello St. plumbers.

Se compariamo poi lo St. Bellardii allo St. phumbeus, noi vediamo differenze di altro genere. La regione occipitale dello St. Bellardii è più corta e quindi più verticale e meno rigonfia che non nello $S t$. plumbcus; più lunga invece ne è la regione espansa fronto-mascellare; mentre nello $S t$. plumbeus le fosse temporali sono quasi perfettamente circolari, nello St. Bellardii esse sono più depresse e più allungate, il loro margine posteriore sorpassando allo indietro l'occipitale superiore, ciò che non succede pello St. plumbcus, ove l'occipitale superiore è per la sua parte mediana molto più allo indietro di detto margine. Il distacco del rostro dalla parte espansa del cranio ¿ molto più sentito nello St. Bcllardii, i mascellari superiori restringendosi quasi immediatamente al dinanzi dell'angolo anteriore delle ossa iugali e scemando poi pochissimo nella loro espansione a misura che ci accostiamo alla estremiti del rostro, mentre nello St. plumbeus essi non si stringono che di una quantita molto meno considerevole al dinanzi dei giugali, ma scemano poi ancora gradatamente di larghezza fino alla metà anteriore del rostro. Infine la mandibola dello St. plumbeus porta 34 a 35 denti per ramo, quella dello St. Bellardii non doveva portarne che trenta circa ed alquanto più grandi.

Le stesse differenze ad un di presso si osservano allorchè compariamo il cranio dello St. Bellardii con quello dello St. sinensis, il quale ha anzi denti ancor piu piccoli e più numerasi che non lo St. plumbeus.

Se compariamo lo St. Bellardii collo St. rostratus, vediamo come questi abbia la regione occipitale piủ convessa e $\mathrm{i}$ condili meno sporgenti allo indietro, la regione facciale espansa più convessa allo infuori, più larga e, comparativamente alla parte rostrale, più allungata; come il rostro ne sia più largo, meno nettamente separato dalla parte espansa per minor restringimento dei mascellari, sia più pianeggiante, più largo, più corto, più appuntito all'estremità e come porti un minor numero di denti e questi più grossi.

Lo Steno compressus messo in confronto dello St. Bellardii ci mostra la regione occipitale molto più convessa e molto meno salienti allo indietro i condili occipitali, le fosse temporali quasi circolari, quindi collocate molto più allo avanti che nello St. Bellardii; la regione facciale espansa dello St. compressus è molto più allungata ed il suo perimetro più nettamente rettilineo. I due si accordano poi nella forma generalo del rostro e nel netto distacco di questo dalla regione facciale espansa; differiscono poi nuovamente nel volume e numero dei denti, tali organi essendo più grossi e men numerosi nello St. compressus che non nello St. Bellardii.

Finalmente lo St. attcnuatus differisce più che tutti gli altri enumerati dallo St. Bellardii per la forma molto più elevata del cranio, per la regione occipitale ancora sempre molto lunga e convessa, per i condili pochissimo salienti allo indietro, yer la forma straordinaria delle sue fosse temporali non limitate allo insủ da margine 
alcuno e sfuggenti per conseguenza nella regione supero-occipitale, per la posizione bassissima del processo mastoideo, per la minore lunghezza del rostro, la sua minor distinzione dalla parte facciale espansa, il minor sviluppo trasversale degli intermascellari ed il maggiore dei mascellari e per la conconitante e visibilissima minor convessità del rostro stesso, infine per avere la maudibola ricurva in su, anzichè rettilinea, e per portare un maggior numero di denti.

Dai caratteri craniologici risulta adunque come lo Steno Bellardii non possa essere confuso con alcuna delle specie viventi o fossili attualmente conosciute e che sia quindi giustificata la costituzione di una nuova specie, alla quale ho dato il nome di Steno Bellardii e che è, dopo lo St. Gastaldii, la seconda specie del genere trovata nei nostri terreni.

Allumle ed Épistrufeo (fig. 114, 115, 116). - Come già dissi parlando dello St. Gastaldii, quella specie presenta il fatto, eccezionale per il genere, di avere l'atlante isolato dallo epistrofeo. Queste due vertebre sono invece completamente saldate e fuse in un sol corpo nello St. Bellurclii. Il pezzo osseo che ne risulta, e clie è disegnato fig. $101,102,103$, presenta anteriormente le due grandi faccie di articolazione coi condili occipitali separati da una profonda infossatura mediana e da ciascun lato il grosso turbercolo rappresentante il processo trasverso dell'atlante diretto allo infuori e un $10^{\circ}$ allo indietro ed in seguito il tubercolo dello epistrofeo molto più sottile e più marcatamente diretto allo indietro. Anche la parte dell'arco neurale spettante allo atlante è di fianco separata per fessure alte e corte da quella spettante allo epistrofeo, ¿̀ molto più grossa e forte di questa seconda che è ridotta a due porzioni di lamina ossea collocate verticalmente sul margine posteriore della sinostosi. La faccia articolare per la terza cervicale è elittica col margior asse diretto trasversalmente e la faccia inferiore della sinostosi è regolarmente arcata in scuso trasversale con leggiera carena lungo la linea mediana. Il canale neurale è inclinato verso l'aranti e la sua sezione rassomiglia al una foglia di trifoglio i cui lobi siano pochissimo sporgenti. In conclusione, tale sinostosi è costituita afiatto sul tipo di quella che noi rediamo nelle altre specie del genere Steno, lo St. Gastaldii sempre eccettuato.

l'crtebic dorsuli (fig. 117, 118, 119). - Come già espressi precelentemente, non ci vennero conservate altre rertebre cervicali; ne abbiamo invece alcune delle dorsali e fra queste ve n’lia di spettanti alla regione dorsale anteriore come alla posteriore. Le dorsali anteriori hanno un corpo cortissimo, il loro diametro antero-posteriore sendo appena la metà del verticale e molto meno della metà del trasversale. I processi trasversi partono dall'arco neurale e il corpo della vertebra presenta da ciascun fianco e nella seconda metà di sua lunghezza, sull'angolo supero-laterale, distinta faccetta per l'articolazione del capitolo della costa.

I corpi delle vertebre vanno crescendo delle dimensioni longitudinale e verticale a misura che ci avanziamo verso la coda dello animale, e giunti all'ultima dorsale noi troviamo che essa ha un diametro longitudinale che è il più grande clei tre; segue poi il verticale ed il minore è il trasversale, colla avrertenza però che la differenza fra il diametro massimo (il longitudinale) ed il minimo (il trasversale) non giunge a $7 \mathrm{~mm}$., poichè il primo ne misura 47 e 41 l'ultimo. Sul corpo di questa vertebra sono naturalmente scomparse le faccette articolari pel capitolo delle coste e la faccia inferiore 
del corpo è nulla, essendo rimpiazzata da una sporgentissima carena a cui confluiscono per tutta la loro estensione le due faccie laterali. I processi trasversi partono in questa vertebra dalli angoli superiori del corpo. assieme ai pedicoli dell'arco neurale, sono diretti allo infuori e poco allo indietro ed insignificantemente piủ larghi alla estremità che alla origine.

Vercebre lombari (fig. 120, 121, 122). - Nelle vertebre lombari cssendo leggermente scemata la lunghezza, i tre diametri del corpo si trovano essere ad un di presso eguali; i processi trasversi nascono direttamente e superiormente dal corpo della vertebra senza confondersi colla origine dell'arco neurale; essi acquistano un grande sviluppo allo infuori e sono anche un po' diretti allo indietro: non si espandono però significantemente alla loro parte estrema. Il corpo della vertebra è sempre inferiormente carenato come vedemmo per l'ultima dorsale; l'arco neurale ha la forma di un triangolo isoscele col lato trasverso eguale alla metà dell'altezza, e l'apofisi spinosa diretta, come generalmente, allo indietro: tutto questo per le lombari anteriori, mancandoci tutte quelle della regione media e posteriore, come pure le caudali tutte.

L'osle (fig. 123, 124). - Nelle coste, intiere o frammentarie, abbiamo rappresentanti di quasi tutti gli elementi. Vediamo come esse in generale fossero, relativamente alla grandezza dello animale, assai forti e robuste (a differenza di quanto osservasi nello St. Gastaldii), come le anteriori (fig. 123) fossero molto appiattite ed espanse ed avessero una porzione pretubercolare sviluppatissima (fig. 124), come questo elemento capitolare andasse via scemando di lunghezza e grossezza man mano che ci accostiamo alla regione lombare e come la ottava o la nona non ne aressero già più traccia. Infine, dal numero dei pezzi raccolti parrebbe che il numero delle coste da ciascun lato fosse, non già di dodici, come nella maggior parte delle specie di Steno, ma bensi di tredici. Occorre notare in primo luogo come l'esservi una costa di piủ non sarebbe carattere sufficiente per la specie quando si perisi alle oscillazioni di molto più grandi che arvengono nel numero delle coste e delle vertebre fra specie vicinissime di Cetacei ed anche nei limiti di una stessa specie e persino (per le coste) tra un lato e l'altro dello stesso individuo; ed in secondo luogo che può benissimo darsi il caso che uno o due frammenti di minor importanza che non mi parvero più appartenere ad alcuna delle coste state ristorate, e dei quali dovetti per conseguenza tener calcolo nella numerazione, vi appartengano invece e che il numero delle coste rientri cosi nel normale.

Esticmili. - Come manca tutta la regione posteriore della colonna vertebrale, cosi mancano pure completamente tutte le ossa appartenenti alle estremita. Nulla se ne può dunque dire, almeno per ora.

Lumghezza. - Abbiamo calcolata la lunghezza del capo a $60 \mathrm{~cm}$. Ritenendo che questa eguagliasse $\mathrm{i}$ due noni della lunghezza dello intiero scheletro, noi veniamo ad avere per quest'ultimo non meno di $270 \mathrm{~cm}$. dalla punta del muso all'ultima vertebra caudale.

Parlando dello Steno Gastaldii dicemmo già come molte delle sue ossa dimostrassero che egli fu ripetutamente abboccato dai Pesci-cani. Lo stesso convien ripetere per lo Steno Bellartii; il suo scheletro si mostra orribilmente mutilato dai loro denti ed in un mio recente lavoro cercai di dimostrare appunto come le traccie presentate dalle ossa di questo Steno sieno dovute ai morsi degli Squali anzichè alla mano dell'uomo.

15 A. Portis. 


\section{N. 58. Steno Bellardii PoRTIS?}

Mi resta ancora a parlare di alcuni avanzi che riferisco pure al genere Steno. Nella Collezione Gastaldi esistevano alcuni frammenti piccolissimi che ravrisai appartenere alla regione anteriore del rostro di un Delfinide; sono porzioni di mascellari ed intermascellari e la loro forma ed esterna convessità sono affatto analoghe agli stessi caratteri quali si riscontrano nello Steno Bellardii. Alcuni denti spettanti allo stesso individuo ed insieme raccolti avvalorano questa ipotesi, avendo la stessa forma e le stesse dimensioni che quelli della specie citata.

La località precisa in cui questi avanzi furono trovati non è data; però l'osservazione del loro modo di fossilizzazione e di un tantino di argilla aderente agli alveoli mi porterebbe a supporre che provenissero da Savona. Sarebbe importante che il fatto si arrerasse, poichè potremmo concludere allora alla esistenza dello Steno Bellardii tanto in Piemonte quanto in Liguria.

\section{N. 39. Steno sp. (an St. Bellardii?).}

Fig. 91 .

11 Prof. E. Sismonda nella tavola inedita più rolte menzionata nel corso di questo Javoro aveva fatto disegnare sotto il N. 8 la figura in grandezza naturale di un frammento di ramo destro di mandibola di un Delfino, trovato nelle sabbie gialle dell'Astigiana. Lo stesso frammento fu per cura del Gastaldi ridisegnato di poi e la figura inviata al Brandt che la riprodusse (non affatto soddisfacentemente) nelle sue Ergacnzungen, etc. (tav. I, fig. 17-18) e che a pag. 13 la descrisse riferendola dubitativamente ad uno dei Tursiops conosciuti dell'Alta Italia (Cortesii e Brocchii).

Il frammento misura 7 centimetri e mezzo di lunghezza per due e mezzo almeno di altezza; contiene sei alveoli, di cui il primo e l'ultimo vuoti, i quattro intermedii ancora riempiti dai denti. Osservato dal lato interno, si scorge che esso apparteneva alla porzione anteriore del ramo mandibolare, poichè i soli due centimetri e mezzo posteriori sono a superficie convessa e liscia, mentre il resto di sua lunghezza, ruvido e scabro, era occupato dalla lunghissima simfisi che, a quanto se ne vede, dovera protendersi molto al di là dell'odierno punto di rottura dell'osso; sulla faccia esterna dell'osso scorgonsi tre fori nutritizi; il frammento è di qualche millimetro più alto verso l'avanti che allo indietro.

Questo pezzo di mandibola appartenne ad un individuo stravecchio; infatti gli alveoli sono ciascuno perfettamente individualizzati e separati l'uno dall'altro da una lunghezza d'osso di almeno $2 \frac{1}{2} \mathrm{~mm}$., i denti hanno il fondo della loro radice compresso e chiuso e la corona usata fin quasi alla base.

Anzichè di un Tursiops ho ritenuto il presente frammento come di uno Steno e credo che esso sia piuttosto riferibile allo Steno Bellardii (1).

(1) No 59 bis. Steno Bellardii Porr1s? Il 20 gennaio 1881 venni chiamato per visitare sopra luogo, in territorio di llontafia, in pieno dominio di sabbie gialle, un ammasso di fossili che, facondo un taglio 


\section{N. 60. Steno sp.}

La Collezione Gastaldi conteneva ancora sei corpi di vertebre provenienti dalla Collezione Sotteri e stati probabilmente trovati in qualcuna delle numerose località fossilifere dell'Astigiana. Spettano tutte alla regione posteriore della colonna vertebrale e probabilmente vi ha fra di esse l'ultima lombare e le prime caudali: non si può però tanto affermare con sicurezza, essendo esse ridotte ai soli corpi e questi ancora incompleti. Ad ogni modo esse appartennero ad un individuo adulto, le epifisi essendo già ben saldate e fuse coi corpi. L'esame loro e sovratutto quello del loro diametro antero-posteriore notevolmente minore degli altri due mi indurrebbero a credere che queste vertebre abbiano appartenuto ad un individuo di una specie di Steno analoga allo St. Gastaldii per la cortęza delle vertebre caudali, non identica però con essa, avendo questa, come fu piu in alto notato, vertebre caudali in cui il diametro verticale $\dot{e}$ il massimo, a cui segue il trasversale, poi l'antero-posteriore, e le vertebre della Collezione Sotteri di cui è ora questione arendo i due diametri, verticale e trasversale, uguali ed entrambi maggiori dell'antero-posteriore. Questi avanzi hanno poi troppo poca importanza per meritare una nuova denominazione specifica.

\section{N. 61. Steno sp.}

Finalmente faremo ancora menzione di un ultimo pezzo che con ogni probabilità deve essere qui collocato. Consiste nel corpo di una vertebra dorsale (delle prime) trovato molti anni addietro in localita non ben precisamente trasmessa, nelle sabbie gialle dell'Astigiana, assieme ad una serie caudale precedentemente descritta di Bcrardiopsis pliocacnus. Tal corpo di vertebra manca di tutti i processi e delle epifisi. La sua forma e le sue dimensioni tanto assolute che relative ce lo fanno riguardare come avente appartenuto al genere Steno e molto probabilmente alla specie Steno Bellurdii (1).

nel terreno, si era scoperto al un tralto, che mi si era fatto sperare fosse un grosso Cetacco fossile e che mi si manifestò invece come un banco di valve di Perna Soldanii. l'erò la cortese mia gujda, il Cap. Angeleri, nella stessa giolnata venne a sapere che in prossima località una vecchía donna riteneva jresso di sè e conservava come un tesoro un grosso osso da lei trovato al basso di antica trincea stradale ́vicino alla fonte solforosa) e dalla quale era caduio in seguito alla lenta erosione successiva della trincea stessa.

Recatici all'abitazione di questa donna, non mi fu difficile il farmi mostrare il jerzo clso riconobti essere il clanio di uno Steno aftine a quello cho vengo ora di descrivere. La porkione posteriore del cranio è alquanto sciupata e molti tratii sono ancura incrostati di roccia. Le mandibule sono ancora in parte conservate ed aderenti a: cranio per nez\%o della roccia arenacea, la estremita anteriore del rostro e delle mandibole stesse è mancante Da un lato molti denti sono ancora intatti. Tanto potei osservare nel tempo in cui mi trattenevo colla proprietaria. Quando però ne volli trattare l'acquisto pel nostro Museo naufragai contro ad un mutismo ostinato, del quale non ebbi la chiave che qualche tempo dopo, allorch venni a sapere che la proprietaria era gia anteriormente in trattative di vendila dell'oggetto stesso e che tali trattative avevano realmente avuto in seguito e per lei un felice risultato.

Tentai poi di rilevare questo fossile dallo attuale proprietario, od almeno di averlo in comunicazione temporanea per assicurare la determinazione fatta cosi di volo, na urlai in un rifiuto altrettanto inesplicato che colla inventrice e che perdura tutfavia. Egli ò con ?? quindi che io segralo nei dintorni di Montafia il rinvenimento di un secondo cranio appartenente alla specie Steno Bellardii l'ortis (Nota aggiunta durante la stampa).

(1) N $^{\prime \prime} 61$ bis. Delfinoide indet. Molti anni addietro venne racculto a Baldissero Torinese, nelle assise 


\title{
PARTE TERZA
}

SIRENOIDI

\author{
HALITERIDI \\ GENERE FELSINOTHERIUM CAPELL. \\ N. 62. Felsinotherium subapenninum (BRUNo) CAPELL.
}

(= Serresii $M_{\text {. }}$ d. Serr.?).

Nel 1828, nel Pliocene inferiore di Montiglio, venne scarato lo scheletro di un Sirenoide che fu nel 1839 illustrato dal Dott. Domenico Bruno (Vedi nella Bibliografia al $\left.\mathrm{N}^{0} 55\right)$ sotto il nome di Chcirotherium subapennimum. Per la descrizione del Bruno il fossile Montigliese ricevette subito il posto che gli spettava nella serie animale e furono conosciuti tutti i caratteri che egli, nello stato in cui si trovava, potè presentare. Per conseguenza, benchè stato più volte oggetto di studio e di comparazione con altri pezzi dello stesso genere, non si ebbe posteriormente ad aggiungere gran che a quanto era stato detto dal primo illustratore e soltanto nel 1872 il Prof. Capellini, che nel 1865 areva creato il genere Fclsinotherium, raccolse in questo genere anche il fossile di Montiglio. Come originale perfettamente conosciuto ed alla cui bella descrizione data dal Bruno non vi ha niente da aggiungere, mi limito a semplicemente registrarlo in questo mio Catalogo.

\section{N. 63. Felsinotherium subapenninum (BRUNo) Capelt.}

Molti anni dopo la scoperta di uno scheletro di Sirenoide a Montiglio i signori Proff. E. Sismonda e L. Bellardi scavavano, ancora a Montiglio, nel giardino della villa Cocconito, avanzi di un secondo scheletro. Questi vennero trasportati e conservati nel Mreo Geologico di Torino.

superiori del Miocene medio, un moncone di osso che era accompaguato da qualche dente di Squali e di qualche Schizasteride. L'osso è un frammento di costa di un Cefaceo lungo un $67 \mathrm{~mm}$. e largo, dove è più dilatato, $\mathrm{mm}$. 16. È piano su di una sua faccia, assai convesso sulla opposta: lo due faccie separate la margini molto pronunziati ed anzi il superiore quasi tagliente. La costa di cui il moncone facea parte era una delle posteriori ed il moncone stesso dimostra col suo graduale allargamento di esserne stato collocato presso alla estremità distale. Naturalmente è impossibile una determinazione anche di sola famiglia di un pezzo cosi insignificante. Suppongo abbia appartenuto a qualche piccolo Delfinoide.

II pezzo ci mostra poi traccie non dubbie (tagli, ecc.) dello addantamento per parte degli Squali (Nota aggiunta durante la stampa). 
Passando in rivista tutto quello che di questo scheletro si potè raccogliere, vidi con dispiacere che non era possibile il tirarne alcun profitto. I frammenti di vertebre e di coste che abbiamo dinanzi, quantunque assai numerosi, sono tutti troppo incompleti e troppo impastati nella roccia perchè si possa di loro dare una descrizione qualunque; il cranio e tutte le parti delle estremità mancano completamente e solo la natura delle ossa e la forma delle coste ci indicano che noi abbiamo a che fare con un Sirenoide. La dimensione poi di ciascuna parte è la stessa che per quelle dello scheletro primo di Montiglio. È probabile che esso appartenga alla stessa specie di quello.

Noterò come alcune coste di questo scheletro dimostrino, cogli intagli che portano, che l'animale, prima di essere sepolto fra $\mathrm{i}$ depositi marini, venne violentemente spogliato delle carni per opera degli Squali.

\section{N. 64. Felsinotherium sp.}

Or sono due anni, nello scavare una cantina in un piccolo borgo in territorio di Casale MIonferrato, si scoprirono in una fina sabbia argillosa biancastra, completamente identica a quella in cui furono trovati i due Felsinoterii di Montiglio, alcune ossa. Un pezzo di roccia con parte delle medesime venne donato a questo $\mathrm{R}$. Museo Geologico, il resto rimase in posto, mascherato dalla muratura da cui fu rivestita la cantina.

Il pezzo conservato presenta una vertebra caudale e porzione di due altre di un animale che ho tosto riconosciuto non essere altro che un Sirenoide e molto probabilmente un Fclsinotherium. La vertebra più completamente conservata (la posteriore delle tre) appartiene alla porzione anteriore della serie caudale e misura $82 \mathrm{~mm}$. di lunghezza e, sulla faccia posteriore, $57 \mathrm{~mm}$. di altezza e $70 \mathrm{~mm}$. di larghezza. Mostra i processi trasversi molto ben sviluppati ed alquanto più espansi all'estremita ed i tubercoli posteriori di articolazione per l'osso a $V$ pure assai sporgenti ed estendentisi allo avanti in una doppia carena che li congiunge ciascuno al corrispondente tubercolo anteriore. I'arco neurale è completamente mancante.

Le vertebre facevano parte di un individuo affatto adulto e le dimensioni loro fanno supporre che la specie cui tale indiviluo apparteneva possa essere la stessa di quella che forni i due scheletri di Montiglio, vale a dire il Felsinotherium subapenninum (Bruno) CAPELL. (1).

\section{N. 65. Felsinotherium Gastaldii DE Zigno.}

Finalmente, nella ricca Collezione fatta con tanta cura dai signori fratelli Craveri di Bra si ammira l'originale del Felsinotherium Gastaldii (nel nostro Museo ne abbiamo un modello), trovato nel dicembre 1876 nel Pliocene delle colline di Bra.

(1) No 64 bis. Felsinotherium sp.? Due anni or sono venne scoperto a Camino presso Casal-Alonferrato un moncone di costa anteriore lungo un $20 \mathrm{~cm}$. di cui non si può dire altro se non che esso spetta sicuramente ad un Sirenoide e probabilmento ad un Felsinoterio Nota aggiunta duranto la stampa). 
Questo pezzo superbo è stato recentemente e completamente studiato e descritto dal Comm. A. De-Zigno (Vedi $N^{*} 106$ della Bibliografia) ed io non ho nulla da aggiungere a quello splendiclo lavoro illustrativo. Mi limito a ricordare semplicemente in questo mio Catalogo il nome del Felsinotherium Gastaldii.

\section{GONGLUSIONE}

Da tutto quanto precede noi vediamo che il sottordine dei Misticeti è copiosamente rappresentato nei terreni terziarii superiori del Piemonte, molto più scarsamente in quelli della Liguria.

Una piccola specie di Bulenottera visitò le nostre regioni mentre si depositavano i conglomerati miocenici che formano una si gran parte della nostra collina e vi lasciò gli avanzi stati scoperti nel 1864 dal Volpato. Forse ad una piccola Balenoltera sono da ascriversi pure gli avanzi di Cetaceo scoperti nei conglomerati miocenici di Serravalle Scrivia. È probabile che la stretta zona di mare in cui si depositarono i conglomerati miocenici della collina di Torino e della base dello Appennino non fosse abitata che da una sola specie di Misticeti e che a due individui della medesima spettino gli aranzi di MIontolino e di Serravalle.

Ia durante il depositarsi dei terreni pliocenici, e sorratutto dei superiori, il mare che copriva l'odierna valle del Po dovette letteralmente essere invaso dai $\mathbf{M} i$ sticeti, numerosi in specie, numerosi in individui.

Noi redemmo un pezzo che ci fa supporre avere anche da noi vissuto il genere Balaemula. Abbiamo veduto come il genere Balacnoptera fosse rappresentato in Piemonte e Liguria molto probabilmente da cinque diverse specie. Di esse l'una, la Balacnoptera Grastuldii, la più piccola e molto len riconoscibile, lasciò avanzi che accennano a cinque individui distribuiti sia nel Pliocene piemontese che nel ligure; essa è finora limitata all'Alta Italia occilentale.

Della seconda, già da lungo tempo conosciuta in tutta l'Alta Italia fino a Bologna, era già stata più volte affermata l'esistenza anche in Piemonte, non però sufficientemente dimostrata: abbiamo reduto come essa sia più rara nelle argille nostre azzurre, comunissima nelle sabbie gialle dell'Astigiana, come i resti fin qui trovati accennino ad almeno trenta individui diversi, ai quali bisognerd̀ aggiungere tutti quelli scoperti anteriormente al nostro secolo, e dei quali non possiamo figurarci la quantità, e tutti quelli che ancora oggidi sono a mani di contadini che li ritengono come tesori e dei quali celano la possessione. Non passa amno intanto senza che or qua or là non vengano segnalati nuovi avanzi, quando più quando meno significanti, ma quasi sempre appartenenti alla Balaenoptera Cortesii. Questa specie abbondantissima in Piemonte non pare lo fosse altrettanto nella Liguria occidentale, almeno finora non ne conosco avanzi.

Una terza specie gigantesca per lo stretto seno di mare in cui abitava e raggiungente dimensioni di poco inferiori a quelle della Balaenoptera musculus si ag- 
giungera alla fauna dei Misticeti piemontesi, mentre in Liguria una o due specie aventi caratteri intermedii tra le Balene e le Balcnottere accompagnavano la $B a-$ lacnoptera Gastaldii. Arevamo cosi in Piemonte una specie di Balucnula e tre diverse specie di Balaenoptera ed in Liguria sicuramente due ma forse tre specie di Balaenoptera, di cui una identica con una piemontese: in tutto adunque sei e forse sette specie di Misticeti pliocenici. Vista la mole, le facoltà locomotrici di questi animali, abbiamo ragione di dire che il sott ordine dei Misticeti era molto largamente rappresentato nella ristretta zona comprendente le odierne regioni piemontese e ligure.

Chẻ se passiamo al sott'ordine dei Denticeti, troviamo alla base del Miocene medio. lo Squalodon Gastaldii nel calcare di Acqui, e verso la sommità due altri Delfinovinchi; lo Schizodelphis compressus a Barbaresco ed il Champsodtelphis itaticus a Camino; troviamo di più un Delfinino, il Tursiops miocaemus nel calcare di Rosignano.

Certamente non mancarono nel nostro paese i Denticeti mentre si depositavano i sedimenti del Pliocene inferiore; probahilmente vi passarono il Champsodelphis e lo Schizodelphis, ma finora non lo possiamo accertare, non essendosene ancorat trovato alcun avanzo. Fra i Delfinini vi si trovo soltanto lo Stcno Gustulflii.

Per contro i Denticeti paiono moltiplicarsi nel nostro Pliocene superiore e mentre cresce il numero degli aranzi trovati, cresce pure il numero delle famiglie dai medesimi rappresentate.

Cosi: la famiglia dei Fiscteridi è rappresentata da tre generi con una specie a ciascuno, cioè: il Priscophyseter typus, il Physotherium Sottcrii e l'Hoplocetus minor. Di questi tre generi, tutti nuovi pel Piemonte, due sono nuovi anche per gli altri paesi; il terzo è gịa conosciuto con altre specie in Francia, nel Belgio e fors'anco in Italia.

La famiglia dei Zificti è rappresentata con un solo genere ed una sola specie, l'mo e l'altra nuovi pel Piemonte e per la fauna fossile in generale. A questo nuovo Zificte ho dato, per le sue analogie col genere Berardius, il nome di Berardiopsis plioccenus e pare non fosse tanto raro nell'Astigiana, poiche i resti fossili trovati manifestano già la prosenza di almeno tre inclividui.

Finalmente la tribù dei Delfinini, che abbiamo già veduta apparire nel nostro Miocene medio col Tursiops miocaemus, è rappresentata nel nostro Pliocene da due generi. Il primo di essi non comprende che una sola specie già conosciuta nel Pliocene superiore della restante Alta Italia, il Tursiops Cortesii; l'altro comprende invece almeno due specie particolari al Piemonte; di esse, la prima, lo Steno Gastaldii, già stata descritta ed illustrata dal Brandt, la seconda, descritta per la prima volta in questo Catalogo, ricevette il nome di Steno Bellardii. Tutte le specie, dalle quali viene in Piemonte rappresentata la tribù dei Delfinini, lasciarono nei nostri terreni avanzi che indicano la presenza di più individui per ciascuna e ci inducono i sperare che nuovi scheletri abbiano a venir trovati.

Noterò incidentalmente come, allo infuori di una specie ancor dubbiosa di Steno, niuno dei Denticeti pliocenici piemontesi sia finora conoscinto in Liguria.

L'ordine poi dei Sirenoidi è rappresentato da due sole specie appartenenti allo stesso genere ed entrambe finora proprie esclusiramente al Pliocene piemontese. Esse sono: $1^{\circ}$ il Felsinothcrium subapenninum (BRuNo), del quale già si trovarono avanzi 
indicanti la presenza di almeno tre (1) individui; $2^{\circ}$ il Felsinotherium Gastaldii, del quale finora non venne trovato che lo stupendo esemplare di Bra.

A rendere poi visibile con un solo colpo d'occhio come sia estesa e distribuita la fauna dei Hammiferi marini fossili del Piemonte e della Liguria, ho cercato di riunirla nel seguente quadro:

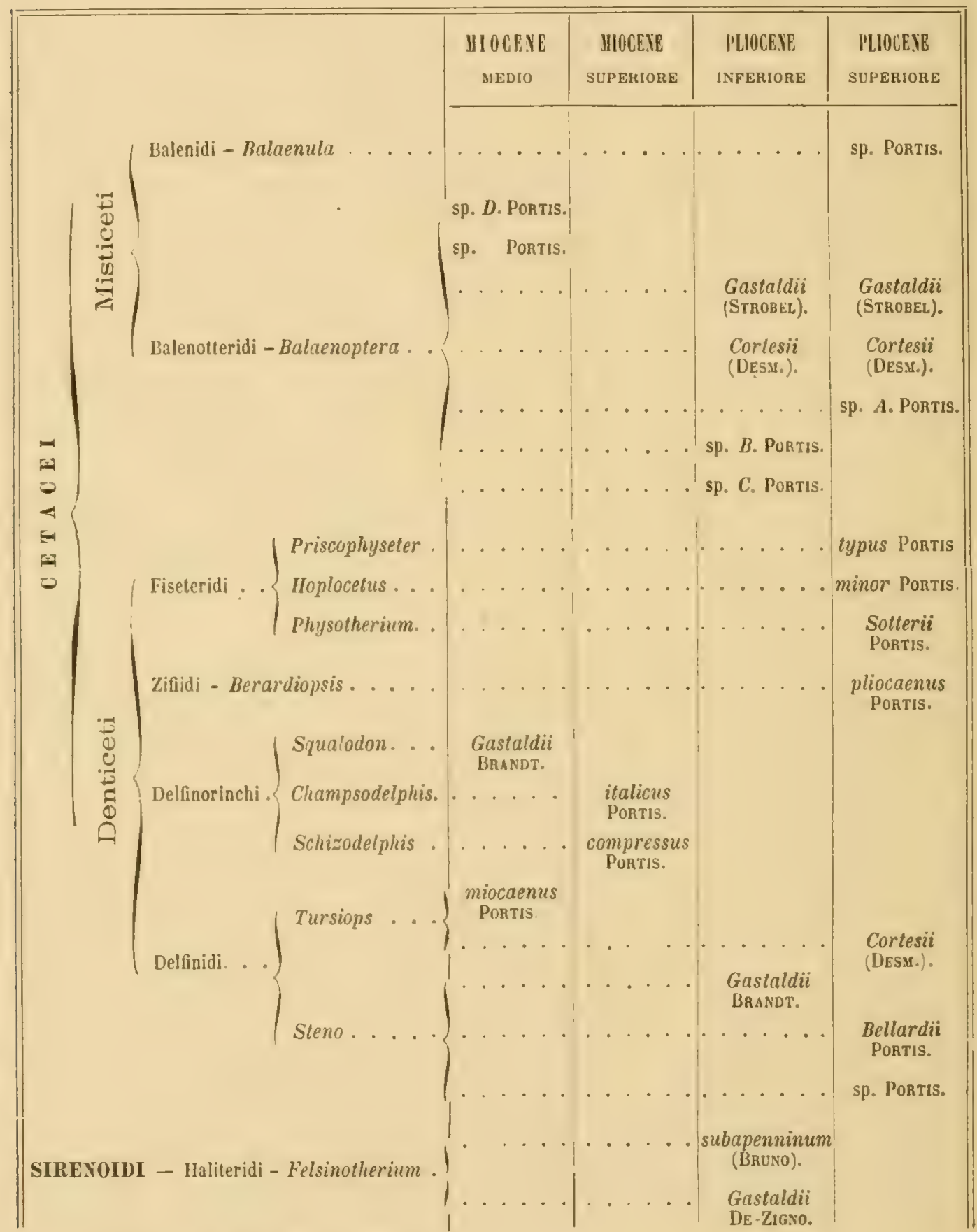

Torino, il 24 aprile 1883 .

Alessandro Portis.

(1) Quattro se contiamo l'avanzo indicato al $\mathrm{N}^{6} 61$ bis. 


\section{ORDINE DELLE FIGURE}

Fig.

1. Vertebra cervicale di Bulacmula veduta dalla faccia anteriore; dimensione $1 / 4 . \ldots . . . . \mathrm{N}^{\circ} 1$ Pag. 1:

2. La stessa veduta di fianco; $1 / 4 \ldots . . .$. ... il. " iri

3. Avanzi del teschio di Balaenoptera Gastaldii veduto dal di sopra; $1 / 4$. . . . . . . " 2 " 22

4. Lo stesso veluto dalla faccia inferiore; $1 / 4 . . .4$ id. " in

5. Lo stesso veduto di fianco; $1 / 4 \ldots . . . . .4$ id. " in

6. Lo stesso reduto dalla faccia posteriore; $1 / 4$. . " id. " ivi $6^{\text {bis. }}$ Cassa timpanica destra di Balcanoptera Gastaldii veduta dalla faccia esterna; $1 / 2 . . . . .3$ ill. " $2: 3$

$6^{\text {ter. }}$. La stessa veduta dalla faccia inferiore; $1 / 2 .$. " il. " ir:

$6^{\text {quater }}$. La stessa reduta dalla faccia interna; $1 / 2 .$. . id. " ir

7. Mandibola destra (Bal. Gastaldii) reduta dal di

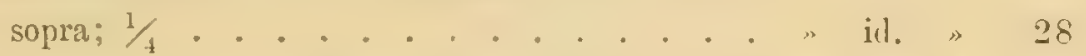

8. La stessa veduta dalla faccia interna; $1 / 4 . .$. i.l. " ivi

9. Porzione posteriore della stessa, veiluta dalla faccia

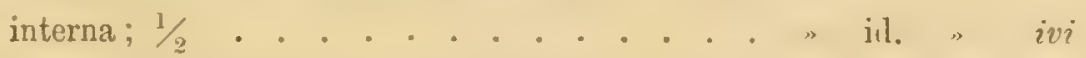

10. La stessa veduta dalla faccia posteriore; $1 / 2$. . " id. " ivi

11. Porzione anteriore della stessa veduta dalla faccia

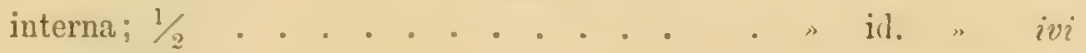

12. La stessa veduta dalla faccia anteriore; $1 / 2 . .$. . id. " ivi

13. Atlante veduto dalla faccia anteriore; $1 / 4$. . " i1. " 31

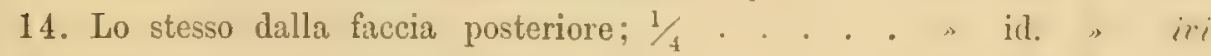

15. Lo stesso veduto di fianco; $1 / 4 . . . . . .7$ il. " iri

16. Seconda vertebra cervicale veduta dalla faccia

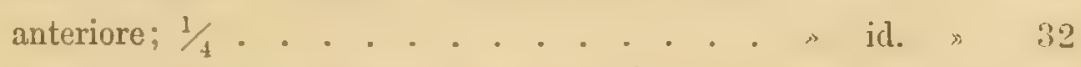

17. La stessa veduta dalla faccia posteriore; $1 / 4 .$. . " id. " iri

18. La stessa veduta di fianco; $1 / 4 . . . . . .3$ id. , ini

19. La quarta vertebra cervicale veduta dalla faccia anteriore $1 / 4$. . . . . . . . . . id. ” 33

20. La stessa veduta di fianco; $1 / 4$. . . . . » id. » ivi

16 A. Portis. 
Fig.

21. Vertebra lombare veduta dalla faccia anteriore; $1 / 4 \mathbb{N}^{\circ} \geq P a g . \quad 33$

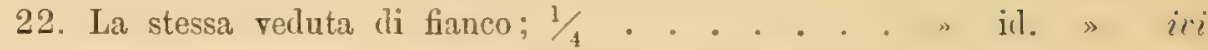

23 . Vertebra candale veduta dalla faccia anteriore: $1 / 4$, il. " ivi

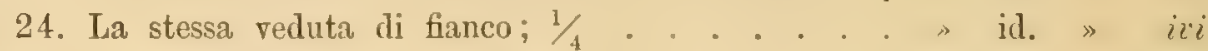

25. Una delle coste anteriori $\left(2^{\mathrm{a}}\right) ; 1 / 4 . .+. .3$. . . . ” 34

26. Una delle coste mediane $\left(4^{a}\right.$ o $\left.5^{a}\right) ; 1 / 4 . . . . "$ il. $\gg$ i i i

27. Frammento di osso joide (corno posteriore destro) $; 1 / 4 \Rightarrow$ id. > 35)

TAY. $2^{\text {a }}$

28. Omero verluto dalla faccia esterna (destro); $1 / 4$ " irl. " ivi

29. Lo stesso veduto dalla faccia interna; $1 / \ldots . . \Rightarrow$ id. * iri

30. Cubito sinistro veduto dalla faccia esterna: $1 / 4$. . il. ” 36

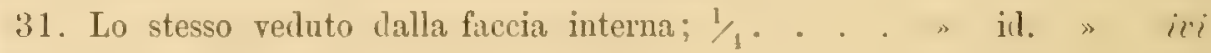

32. Sei ossa falangee; $1 / 4$. . . . . . . . " il. » ivi

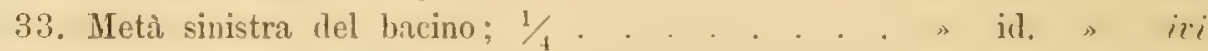

34. Prima costa (bifida all'estremita prossimale) di

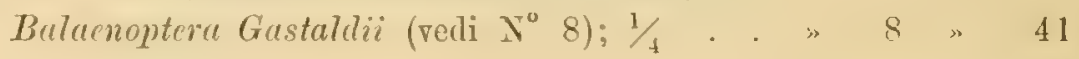

35. Plesiocetus Cortesii. Residuo del cranio veduto

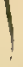
dal di sopra; $1 / 4$

!)

42

TAY. $3^{a}$

3i. Lo stesso verluto dal di sotto: $1 / 1$

37. Lo stesso veduto di fianco; $1 / 4$.

38. Lo stesio veduto di dietro; $1 / 4$

39. Cassa timpanica sinistra veduta dalla faccia esterna; $1 /$

iil. $\gg \quad i v i$

40. La stessa veduta dalla faccia superiore; $1 / 2$. .

41. La stessa veduta obliquamente dalla faccia interna e dal bordo inferiore; $1 / 2 \ldots . . . .$.

42. Ramo mandibolare sinistro veduto dal di sopra; $1 / 4$,

TAv. $4^{\mathrm{a}}$

43. Lo stesso veduto dalla faccia interna; $1 / 4 \ldots .$.

44. Porzione posteriore dello stesso veduta dalla faccia internil: 1

45. La stessa veduta dalla faccia posteriore; $1 / 2$. .

41. Porzione anteriore dello stesso vecluta dalla faccia internia: $1 / 2$.

47. La stessa veduta dalla fociu anterione; $1 / 2=0$

id. » iri

iil. " iri

iil. $\gg 44$

iil. ili

id. " iri

ill. » ili

ill. $"$ ir ;

id. " iri

iil. " iri

jd. " iri

iil. " iri

48. Atlante veduto dalla faccia anteriore; $1 / 4 . .3$.

49. Lo stesso veduto dalla faccia posteriore; $1 / 4$. . " id. " iri

50. Lo stesso veduto di fianco; $1 / 4$. . . . . " ill. " iri

TAV. $5^{a}$

51. Seconda vertebra cervicale veduta dalla faccia

52. Seconda e terza cervicale vedute di fianco; $1 / 4$

53. Terza cervicale veduta dalla faccia posteriore; $1 / 4$

id. $\Rightarrow 47$

il. $\rightarrow 47-48$

54. Sesta cervicale reduta dalla faccia anteriore; $1 / 4$. " id. " iri

55. La stessa veduta di fianco; $1 / 4$. . . . . . " il. " iri 
56. Vertebra dorsale veduta dalla faccia anteriore; $1 / 4$ N" $9 \operatorname{Pag}, 48$

57. La stessa veduta di fianco; $1 / 4$...... " id. " iti

58. Una delle coste anteriori $\left(2^{\mathrm{a}}\right)$ veduta dalla faccia

TAv, $5^{a}$

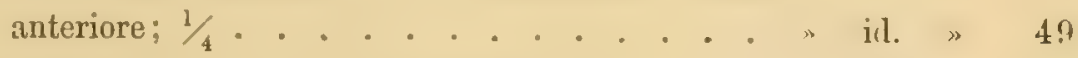

59. La stessa veduta dalla faccia posteriore; $1 / 4 .$. . id. " ine

60. Una delle coste posteriori veduta dalla faccia

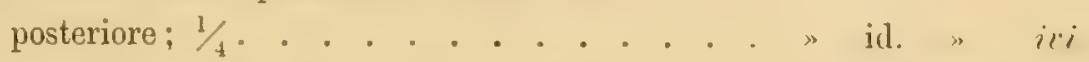

61. La stessa veduta dalla faccia anteriore; $1 / 4 \ldots$. . ill. » ini

62. Vertebra lombare veduta dalla faccia anteriore; $1 / 4$ " id. " ici

63. La stessa veduta di fianco; $1 / 4 \ldots . . . . . "$ id. " ". . ini

64. Scapola destra di Montafia veduta dalla faccia

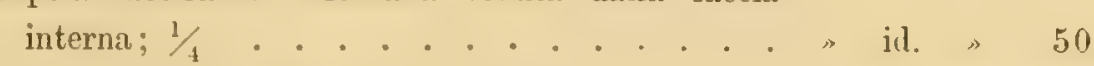

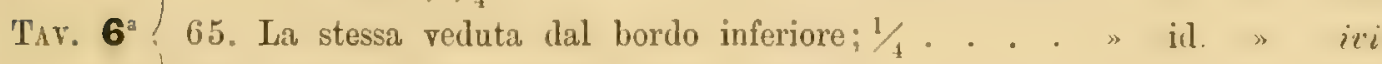

66. Ia stessa veduta dalla faccia esterna...... id. " ivi

67. Una delle vertebre caudali anteriori dello scheletro di Ca-lunga veduta dalla faccia anteriore; $1 / 4 \ldots$. 11 » 56

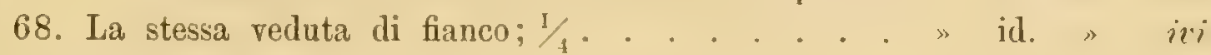

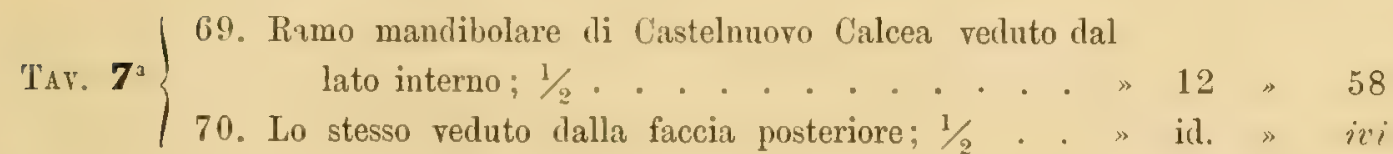

71. Omero destro veduto dalla faccia interna; $1 / 4 .$. " id. " ini

72. Lo stesso veduto dalla faccia esterna; $1 / 4 . .$. .

73. Osso a $V$ (emapofisi) veduto di fianco; $1 / 4 \ldots . . \cdots 28$ » 13.3

74. Cubito sinistro veduto dalla faccia interna; $1 / 4 . " 35$ ” 35

TAv. $\mathbf{6}^{\mathrm{a}}$

75. Lo stesso veduto dalla faccia esterna; $1 / 4 . .$. . " il. » ivi

76. Falange dello scheletro di Montafia; $1 / 4$. . » 9 » 51

$77 a$ e $b$. Due casse timpaniche arenti probabilmente appartenuto ad uno stesso individuo, vedute dalla faccia interna; $1 / z$. . . . . . . . " 31 " 63

78. Osso petroso destro veduto dalla faccia esterna; $1 / 2$ » 32 " 64

79. Vertebra caudale della Balenottera $C$ di Sarona veduta dalla faccia posteriore; $1 / 4$. . . \$ 33 , 69

80. I a stessa veduta di fianco......... ". icl. " iri

81. Cassa timpanica della Balenottera delle fornaci di Savona veduta dal lato esterno; $1 / 2$. . " 38 " 37

82. La stessa verluta dal di sopra e dallo interno; $1 / 2$ " id. " ini

83. La stessa veduta dal di sotto e dallo interno; $1 / 2$ " il " iri

84. Sinostosi cervicale di Priscophyseter typus veduta

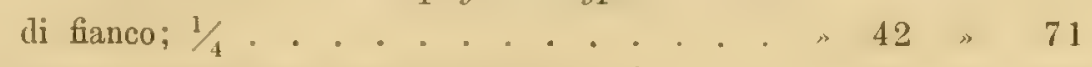

85. La stessa veduta dalla faccia superiore; $1 / 4$. . " il. " ir; 
86. La stessa segata secondo il piano di sinmetria per mostrare le traccie delle vertebre saldate; $1 / 4 \quad \mathrm{~N}^{\circ} 42 \quad \mathrm{Pag} .71$

87. $\}$ Dente di Hoplocetus minor in due posizioni; $1 / 2 \gg 43 \gg 78$

89. Altro dente carioso dello stesso animale; $1 / 2 .$. . id. » 79

90. Altro dente dello stesso segato trasversalmente; $1 / 2$ " id. " iri

91. Dente di Physotherium Sotterii; 1/. . . . " 44 » 81

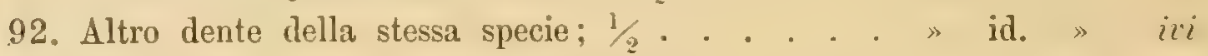

93. Altro dente segato longitudinalmente; $1 / 2 . . . \rightarrow$ id. $>$ iri

94. Porzione di ramo mandibolare di Steno Bellardii veduto dallo esterno; $1 / 2 . . . . ., 59,110$

TAv. 7 7 $^{\mathrm{a}}$. Dieci vertebre caudali di Berartiopsis pliocaenus vedute di fianco; $1 / 4 \ldots . . . . . .4 \quad 45 \% 82$

96. Altra vertebra caudale della stessa specie reduta dalla faccia anteriore; $1 / 4 \ldots . . . ., 46,84$

$97 a$. Vertebra lombare di Champsodelphis? italicus veduta dal di sopra; $1 / 2 \ldots . . . .349$. 490

976 . Vertebra caudale della stessa specie veduta dal

98. La vertebra lombare della fig. 97 a veduta dallo

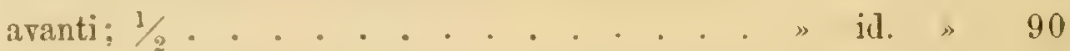

$99 a$. La stessa veduta di fianco ........ . il. » ivi

99 b. La vertebra caudale della fig. 976 veduta di fianco » id. » 91

TAT.

160. Due lastre di argilla fra le quali rimase conservata 101. parte dello scheletro di Schizodelplis com102. Cinque denti di Sclizodelphis compressus raccolti nelle lastre di argilla sunnominate; ad $1 / 1$. .

id. " ivi

103. Teschio mutilato, trovato a Cortandone, del Tursiops

Cortesii, veduto dal di sopra; $1 / 4$. . . . . " 52 " 96

104. Lo stesso veduto dal di sotto; $1 / 4$...... . . id. " ivi

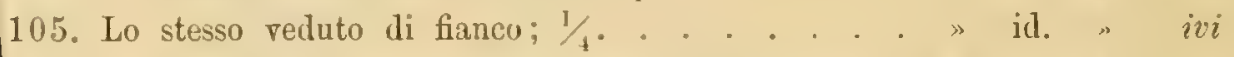

106. Due denti di Tursiops miocacmes di Rosignano. " 55 " 100

107. Teschio, trovato a Bagnasco, di Steno Bellardii

TAF. $\mathbf{9}^{\text {a }}$ veduto dal di sopra; 1/4........ . 57 . 105

108. Steno Bellardii. Teschio veduto dal di sotto; $1 / 4$ » id. » iri

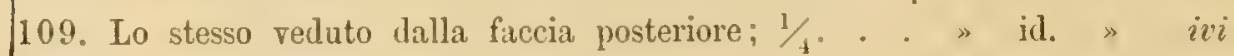

110. Lo stesso veduto di fianco; $1 / 4$. . . . . . " id. " ivi

111. Mandibola destra reduta dal lato esterno; $1 / 4$. . " 55 》 106

113. La stessa veduta dal di sopra; 1/4 . . . . . " id. " ivi

112. Frammento (anteriore) della mandibola sinistra

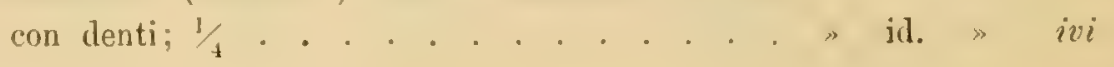


114. Sinostosi cervicale veduta anteriormente; $1 / 4$. . $\mathrm{N}^{\circ} 55$ Pag. 108

11. La stessa veduta posteriormente; $1 / 4$..... " id. " ivi

116. La stessa reduta di fianco; $1 / 4$. . . . . . » id. » ivi

117. Vertebra dorsale veduta anteriormente; $1 / 4 \ldots . . ”$ id. ” ivi

118. La stessa reduta posteriormente; $1 / 4$.... " il. " ivi

TAv. 9 119. La stessa veduta di fianco; $1 / 4 \ldots . . . .0$ id. " ivi

120. Vertebra lombare veduta anteriormente; $1 / 4 .$. . id. » 109

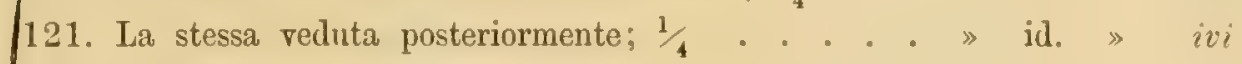

122. La stessa veduta di fianco; $1 / 4$....... \# id. ” ivi

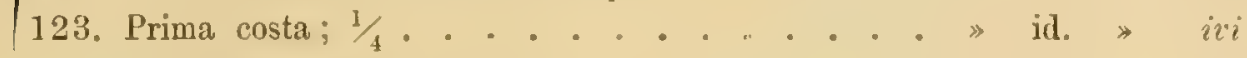

124. Una delle coste anteriori; $1 / 4 \ldots . . . .$, id. » iri 

1. Re Alte de di Garin, $\mathcal{C}$
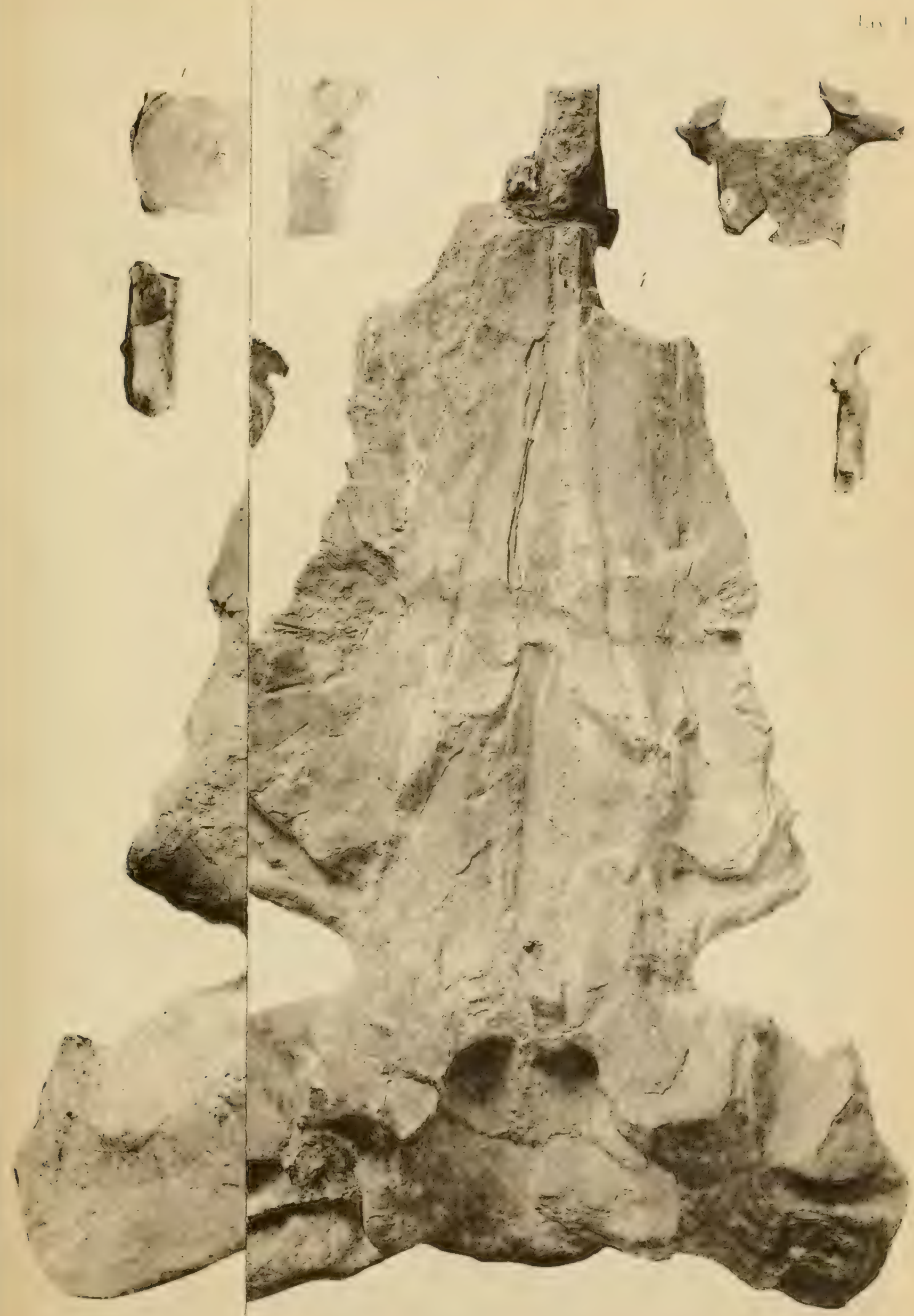



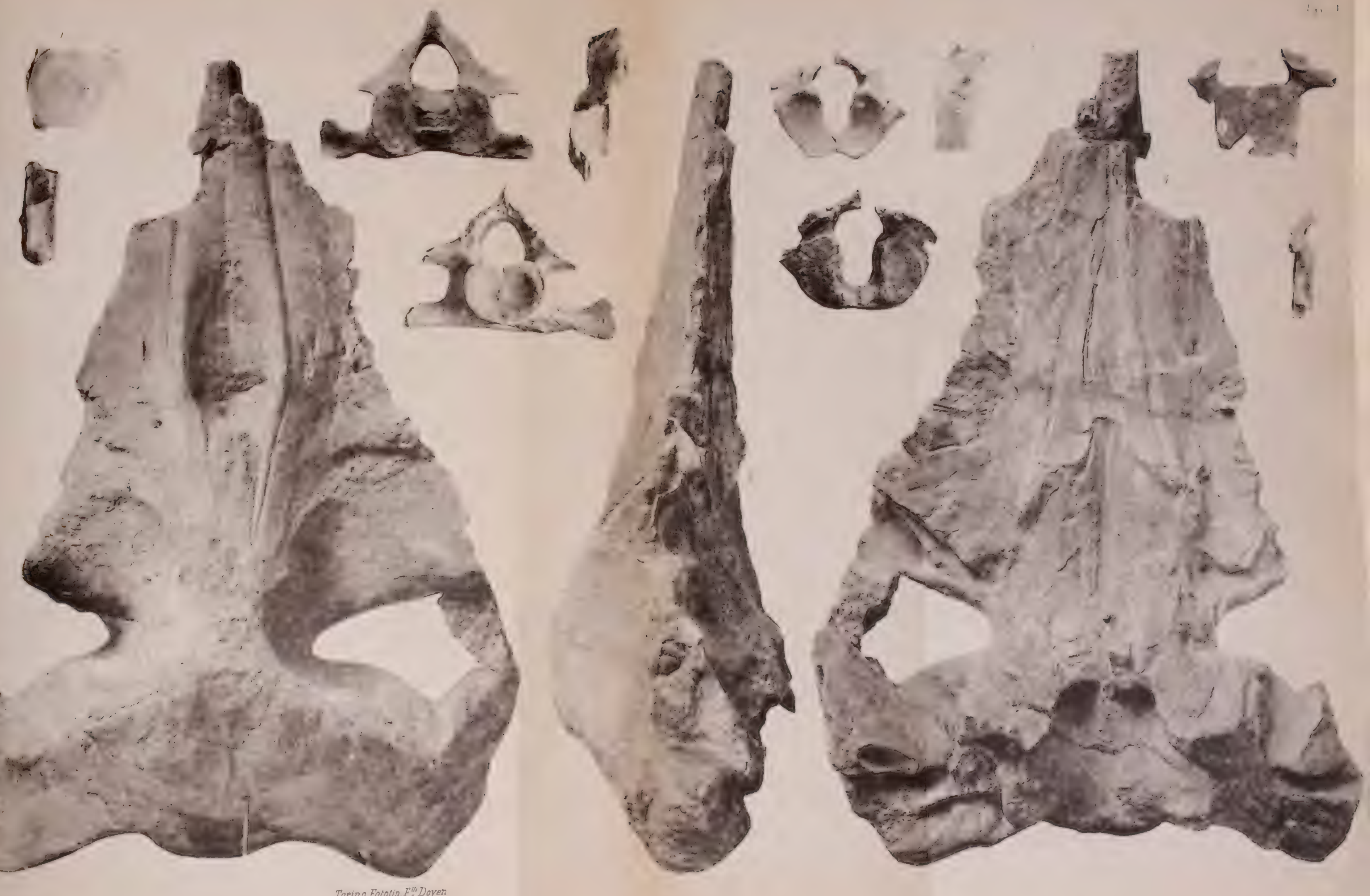



Titv. II.

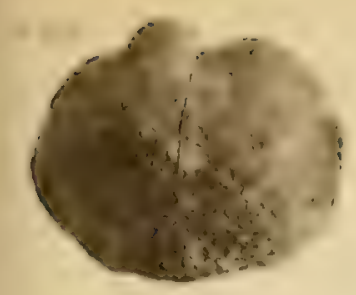

1. III.

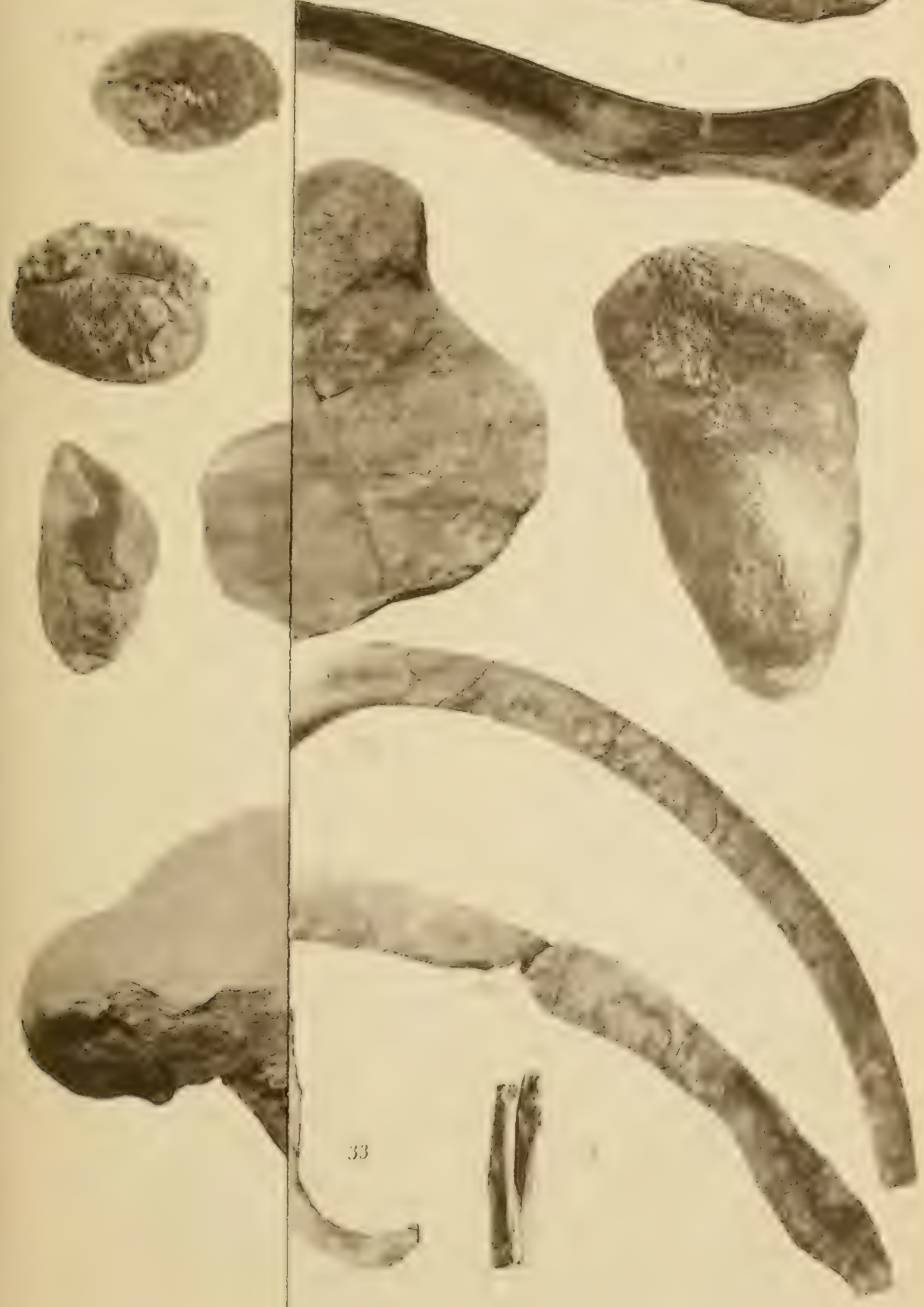





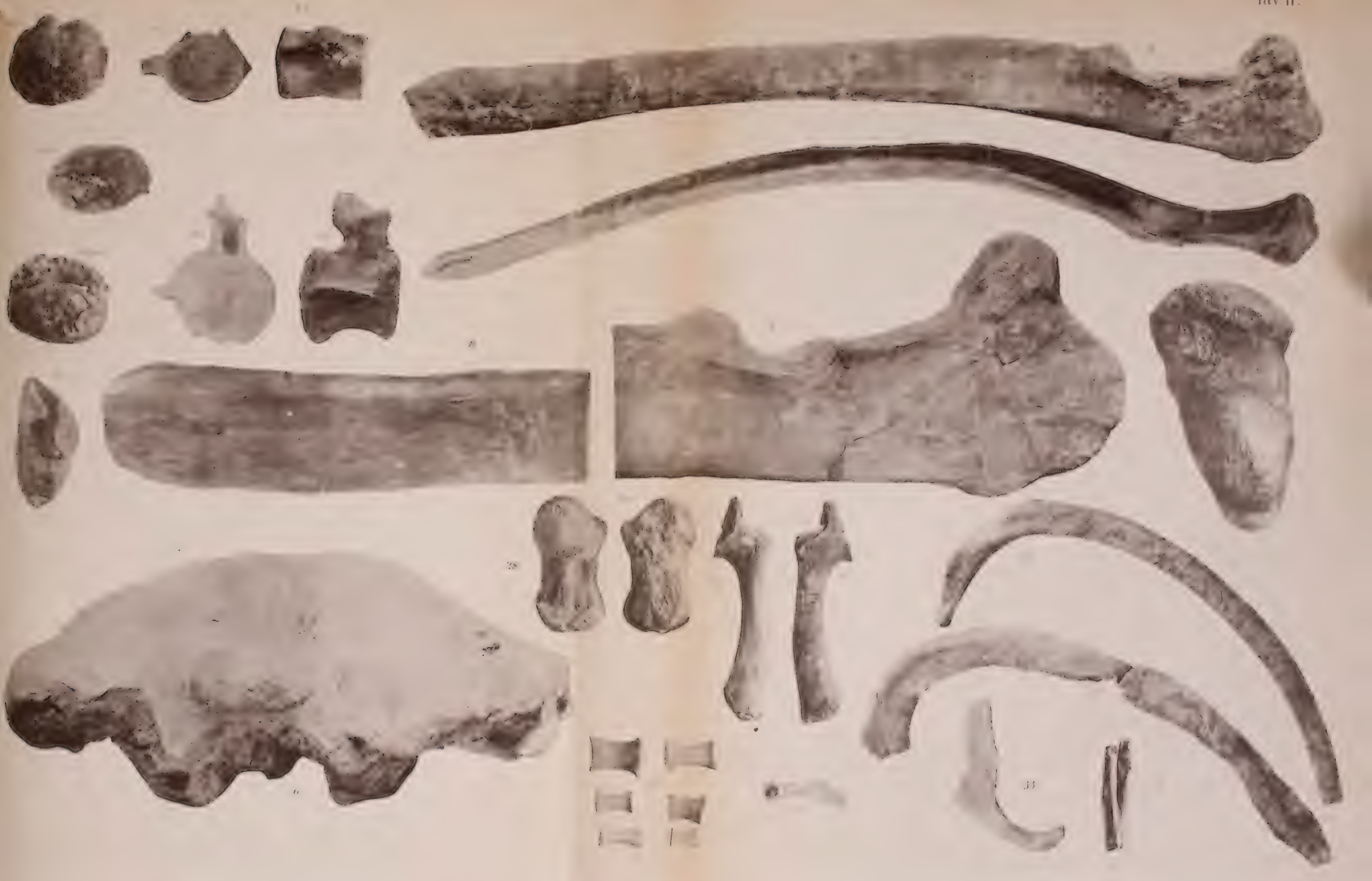





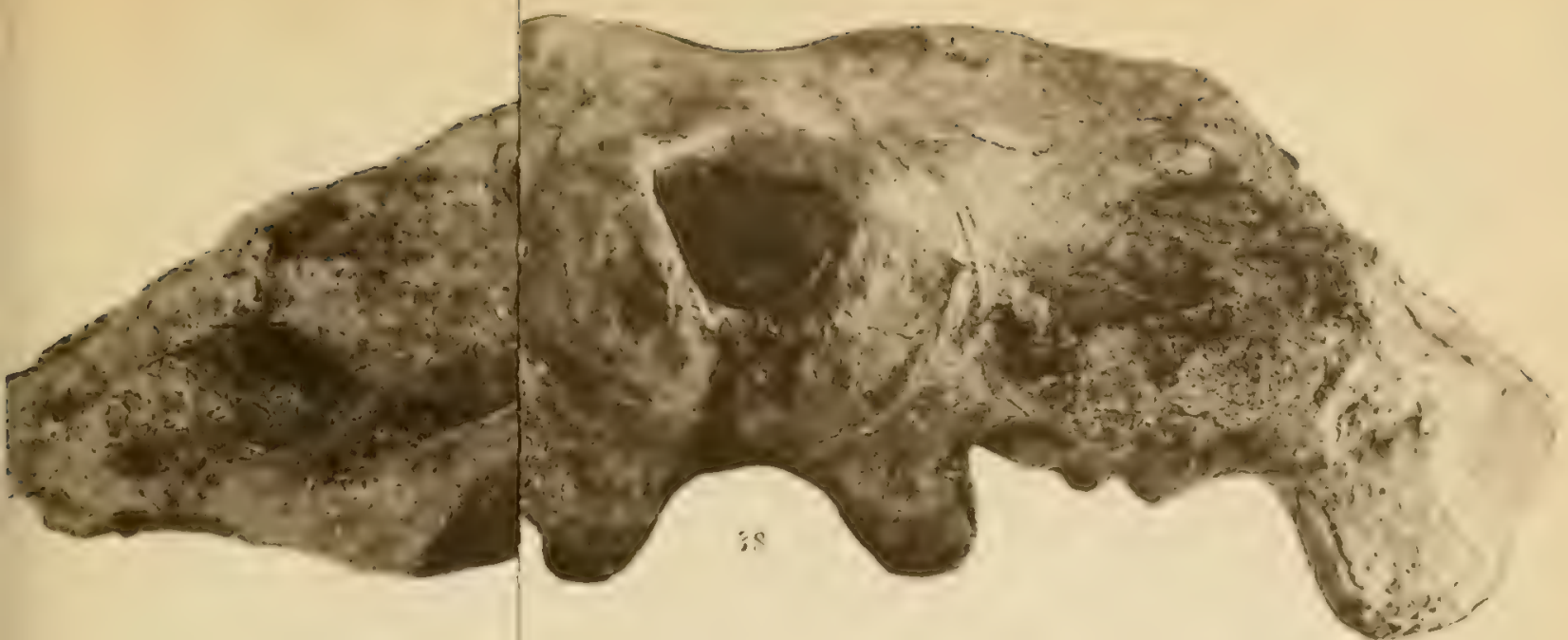

T:I) 111.

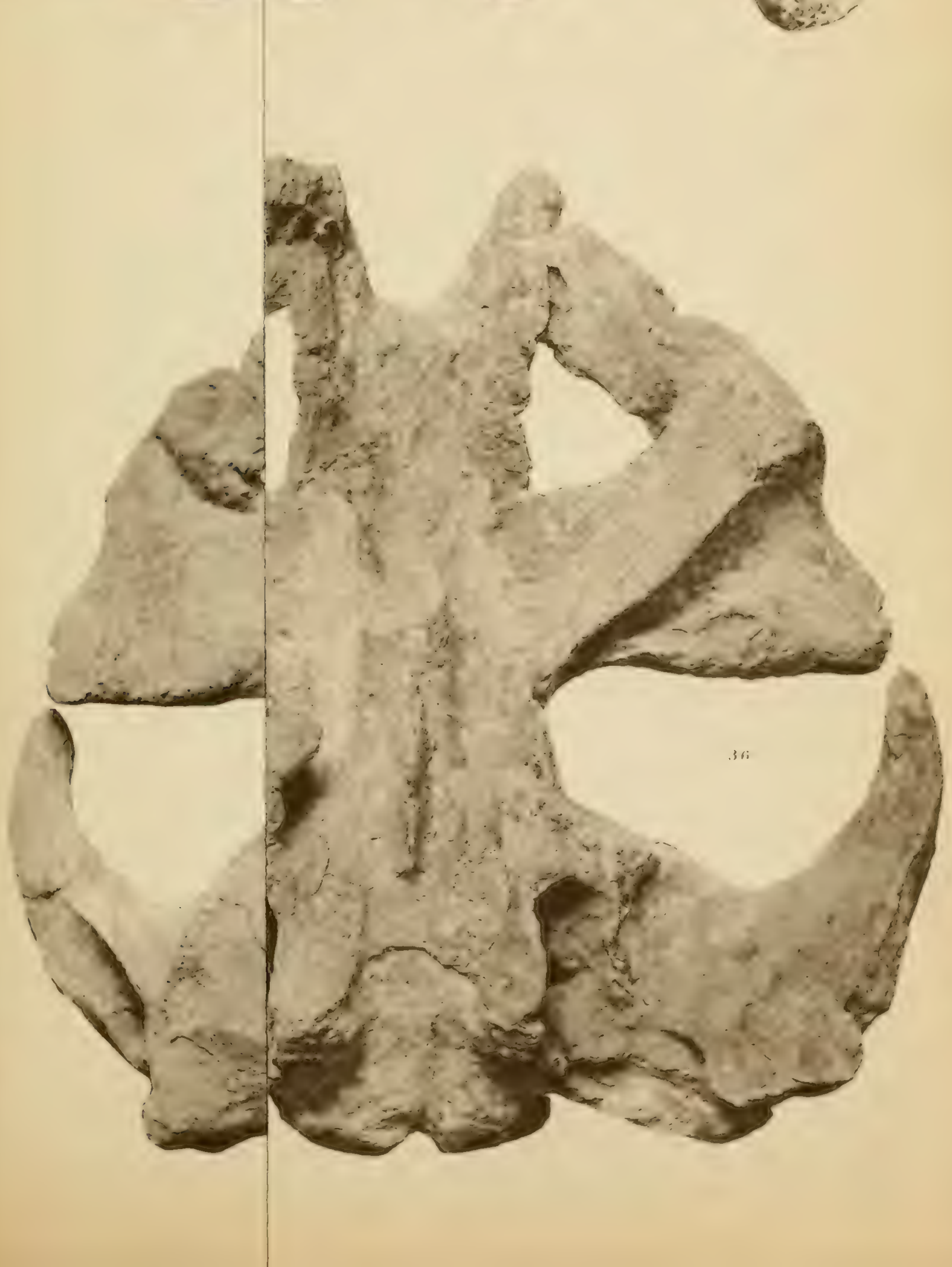





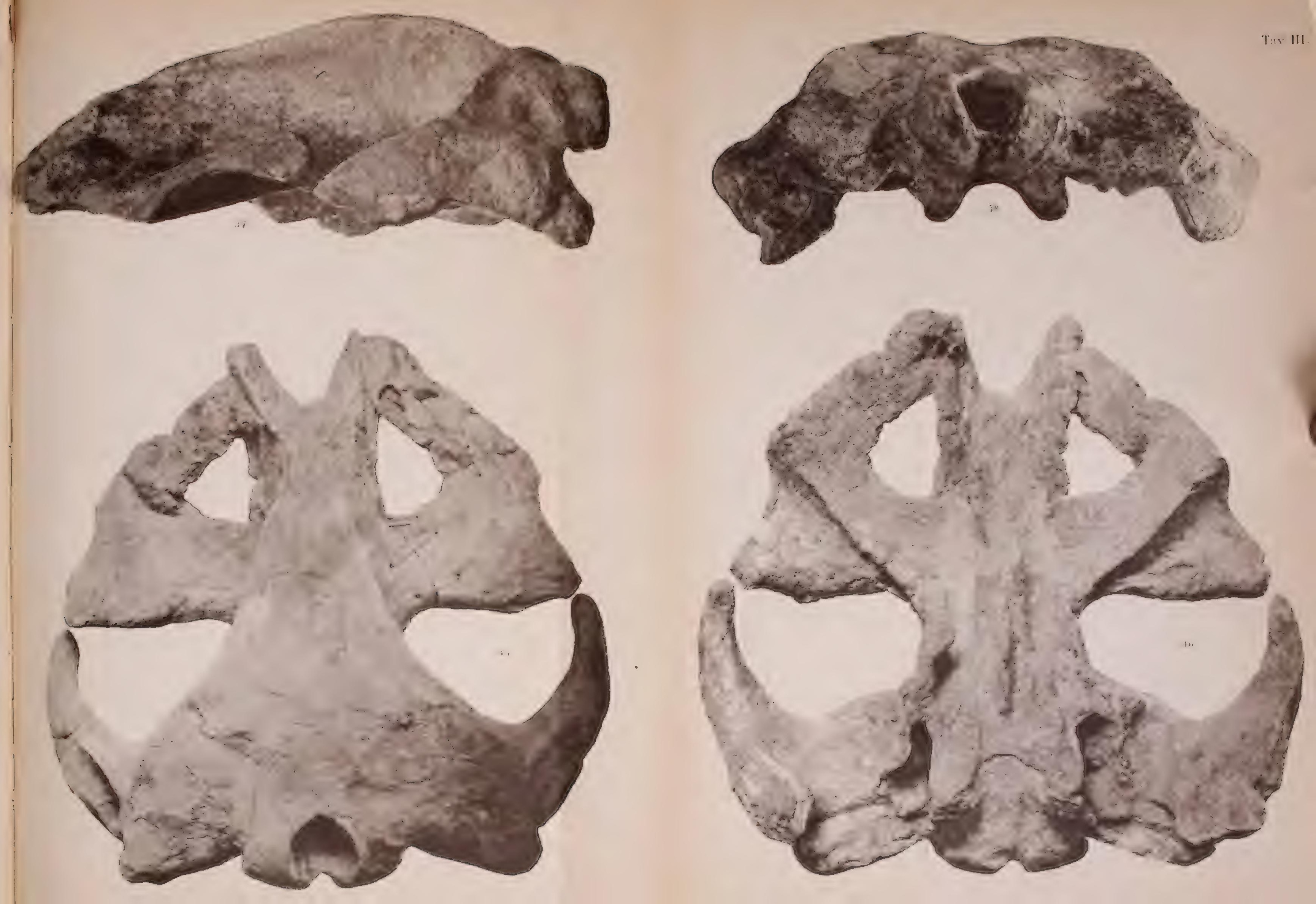




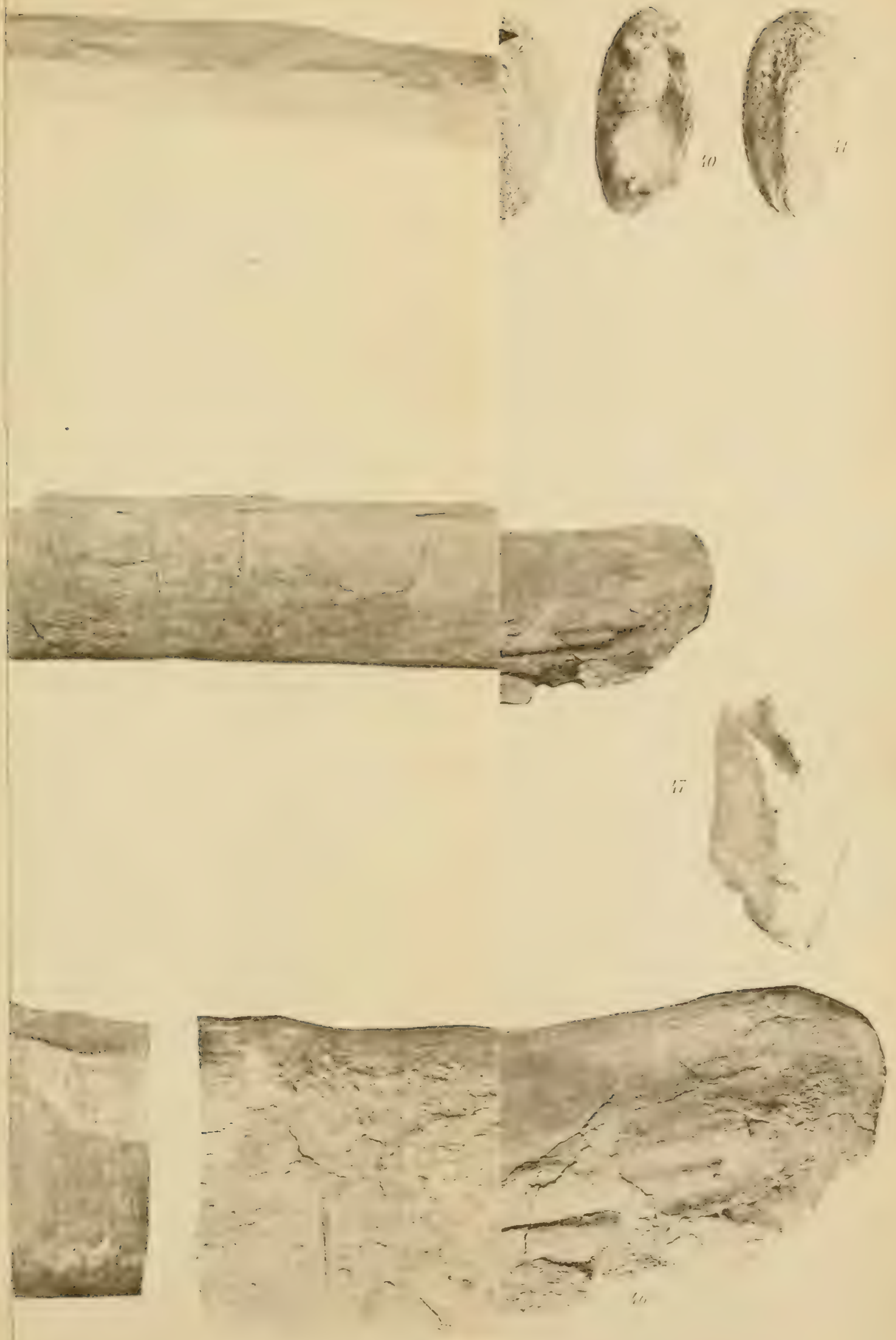



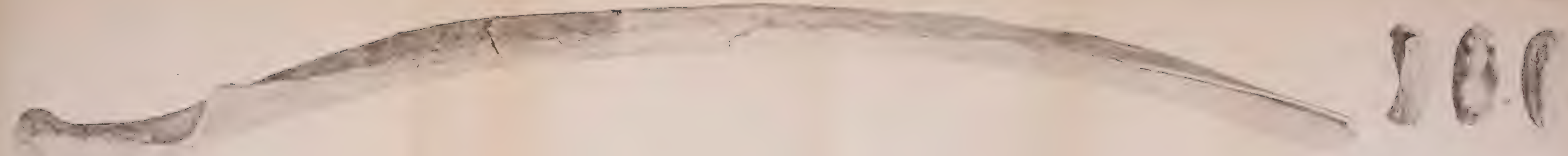

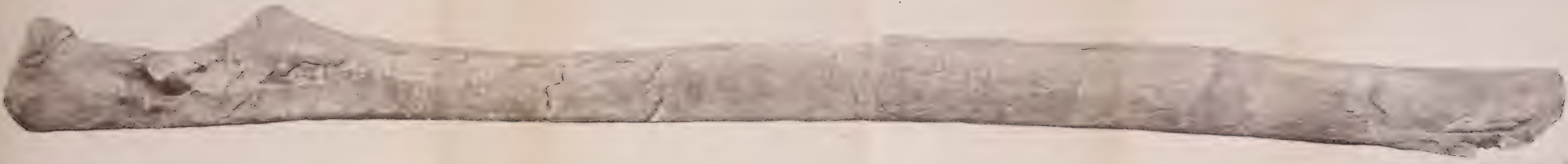
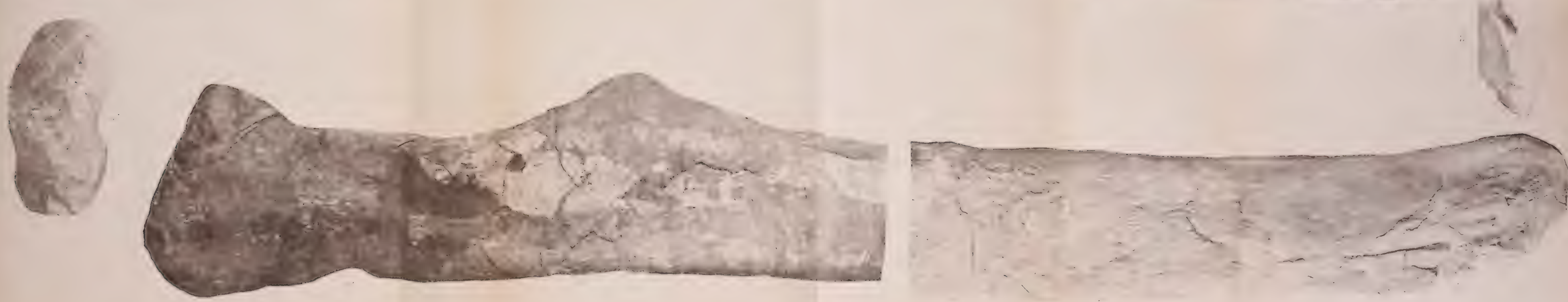

Tay V.
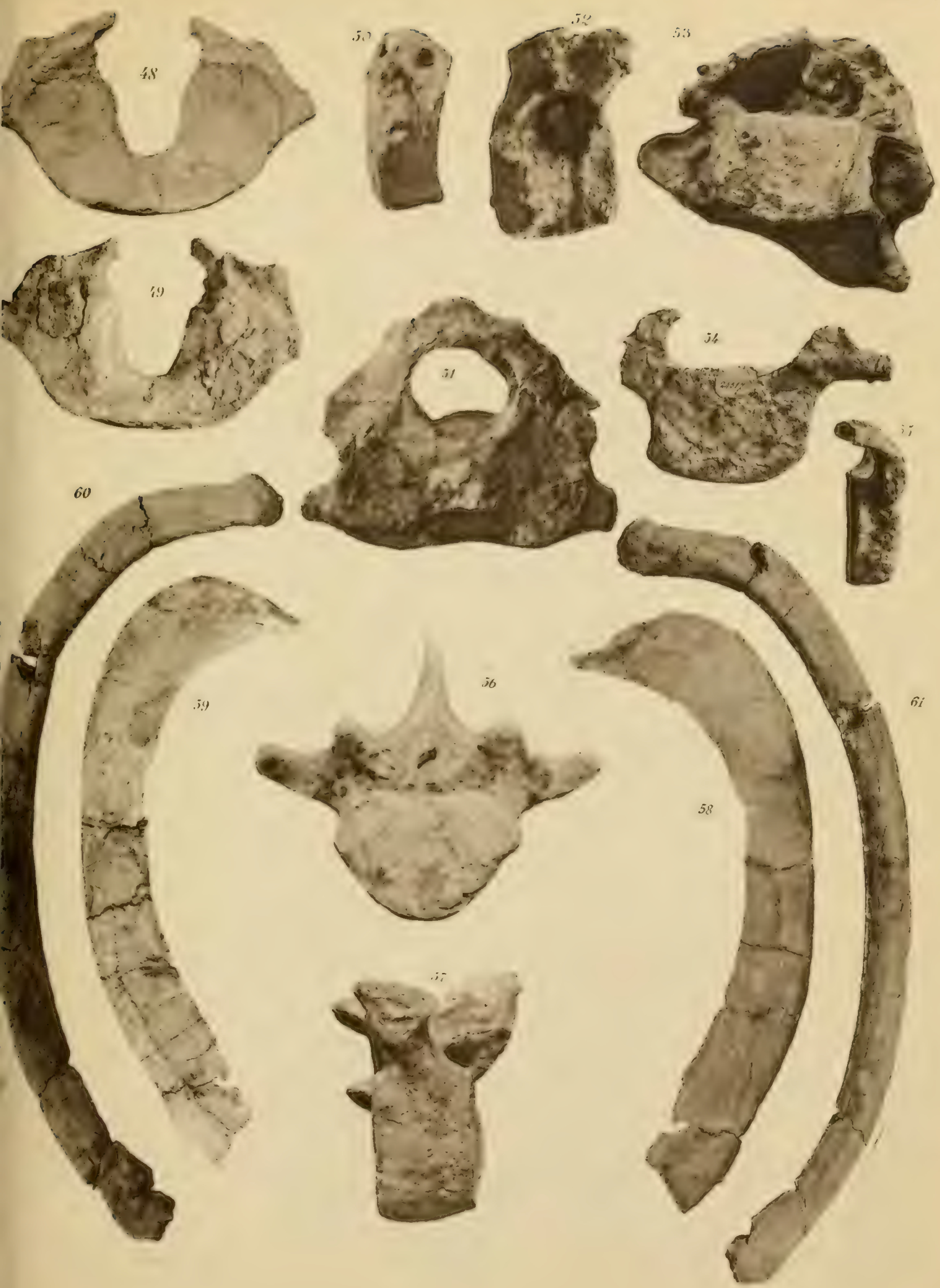




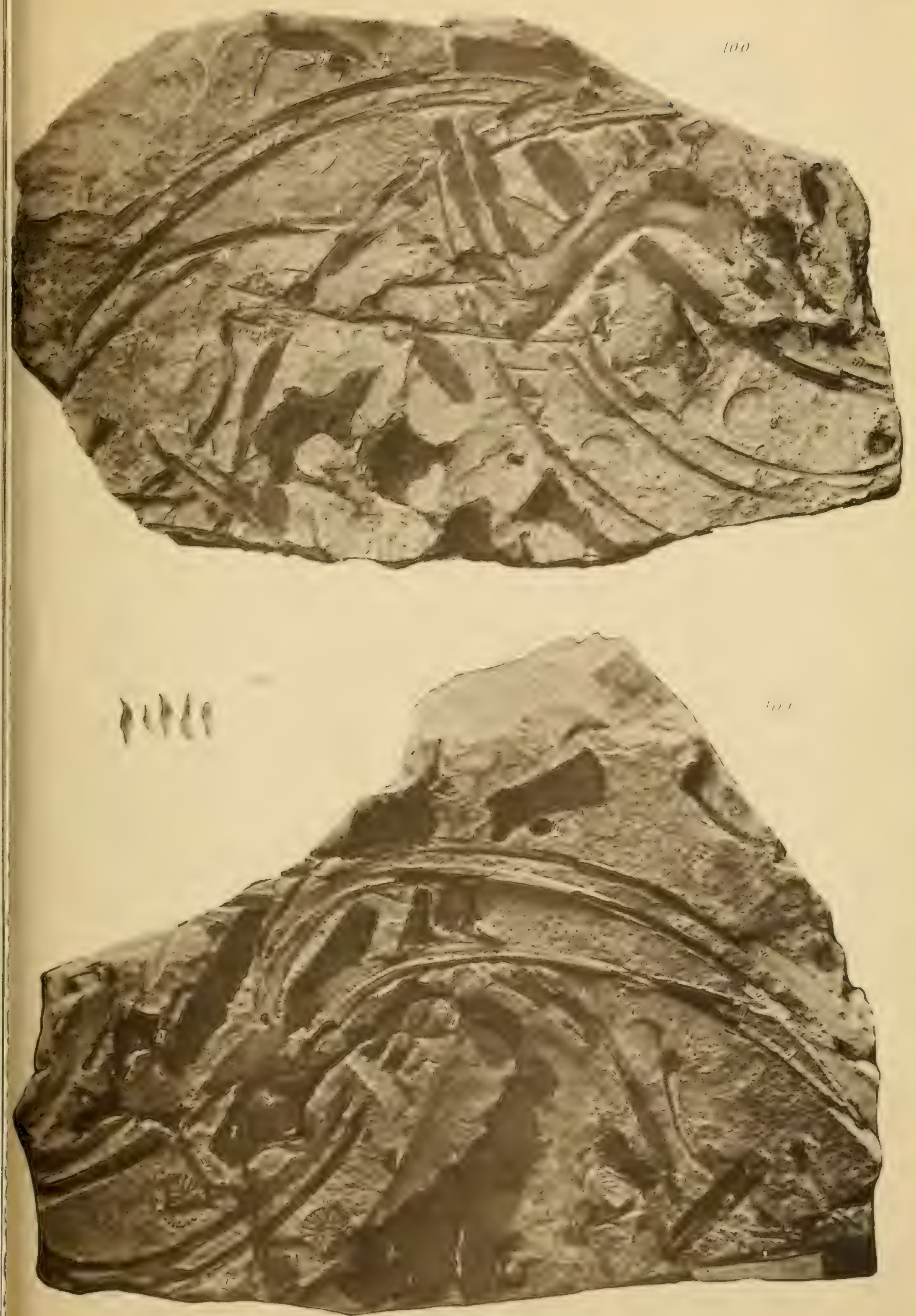




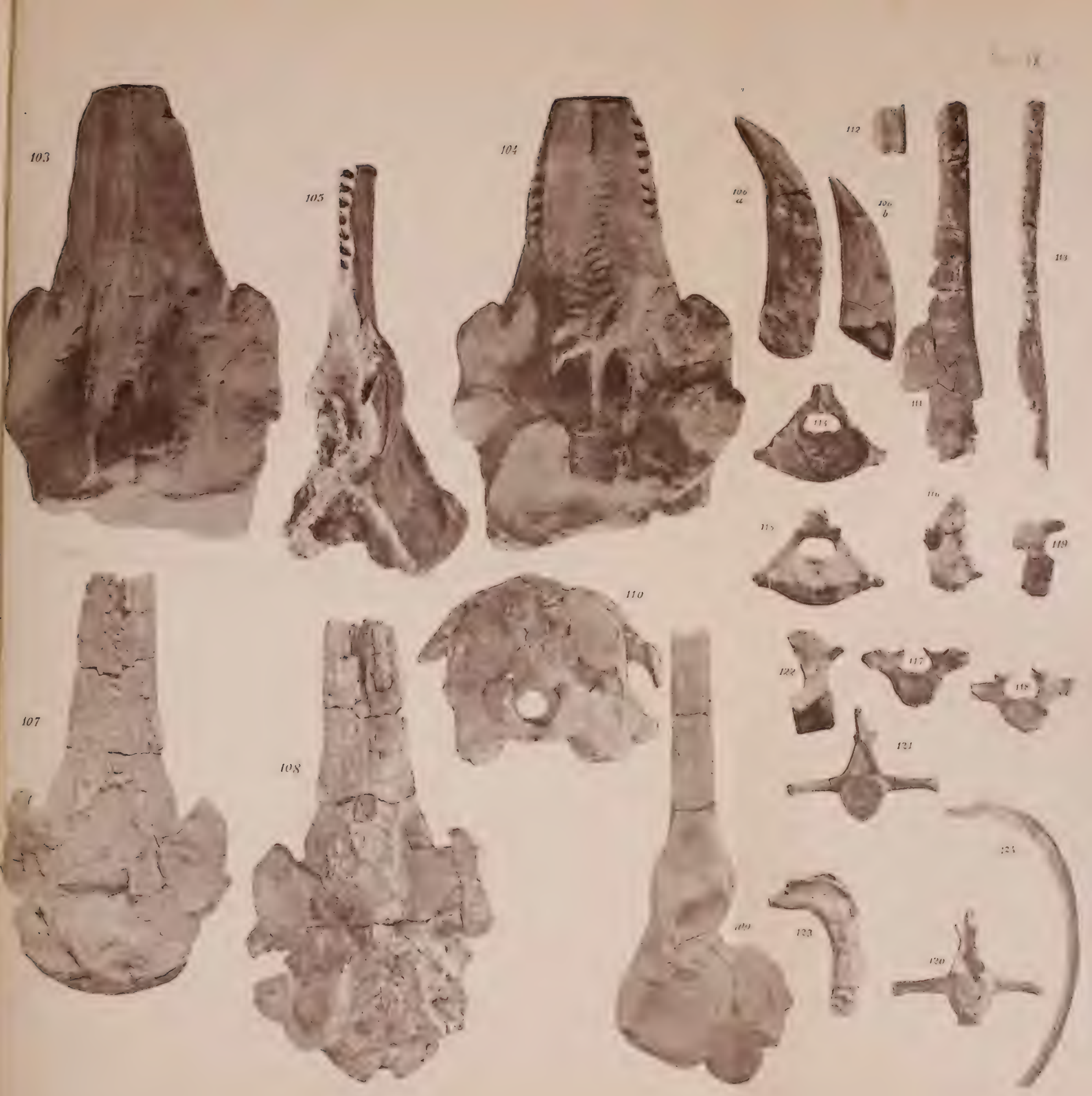








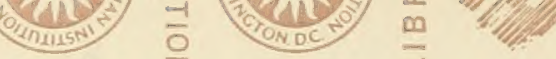

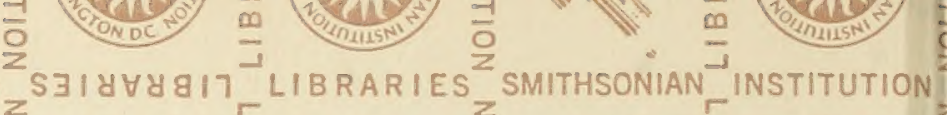
(ㄴ) (n)
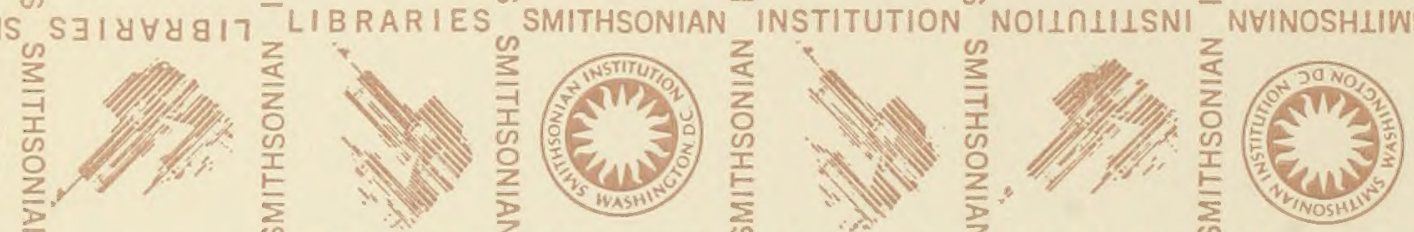

S $\exists 1$ 밤ำ
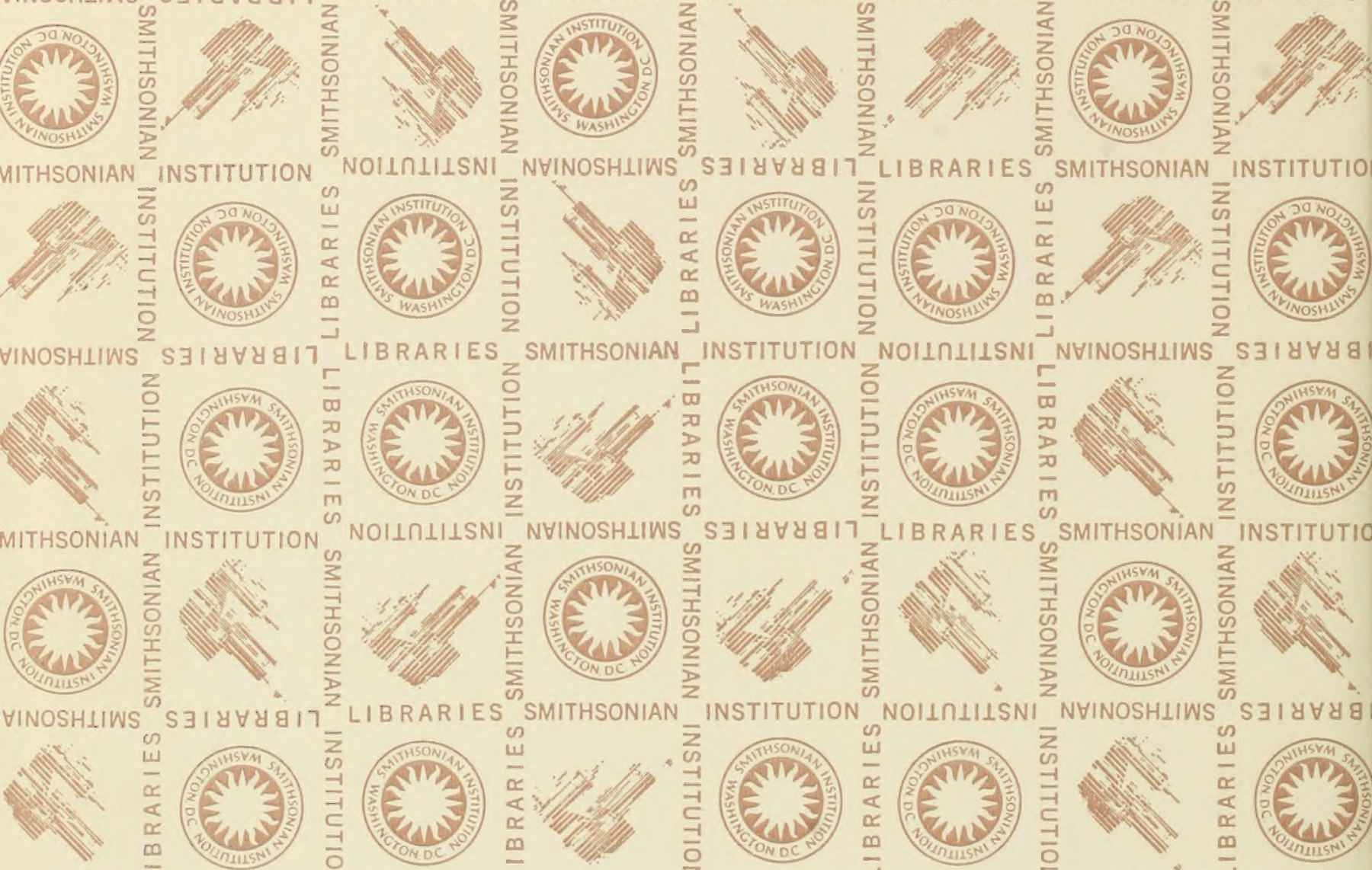

S318ㅁ817
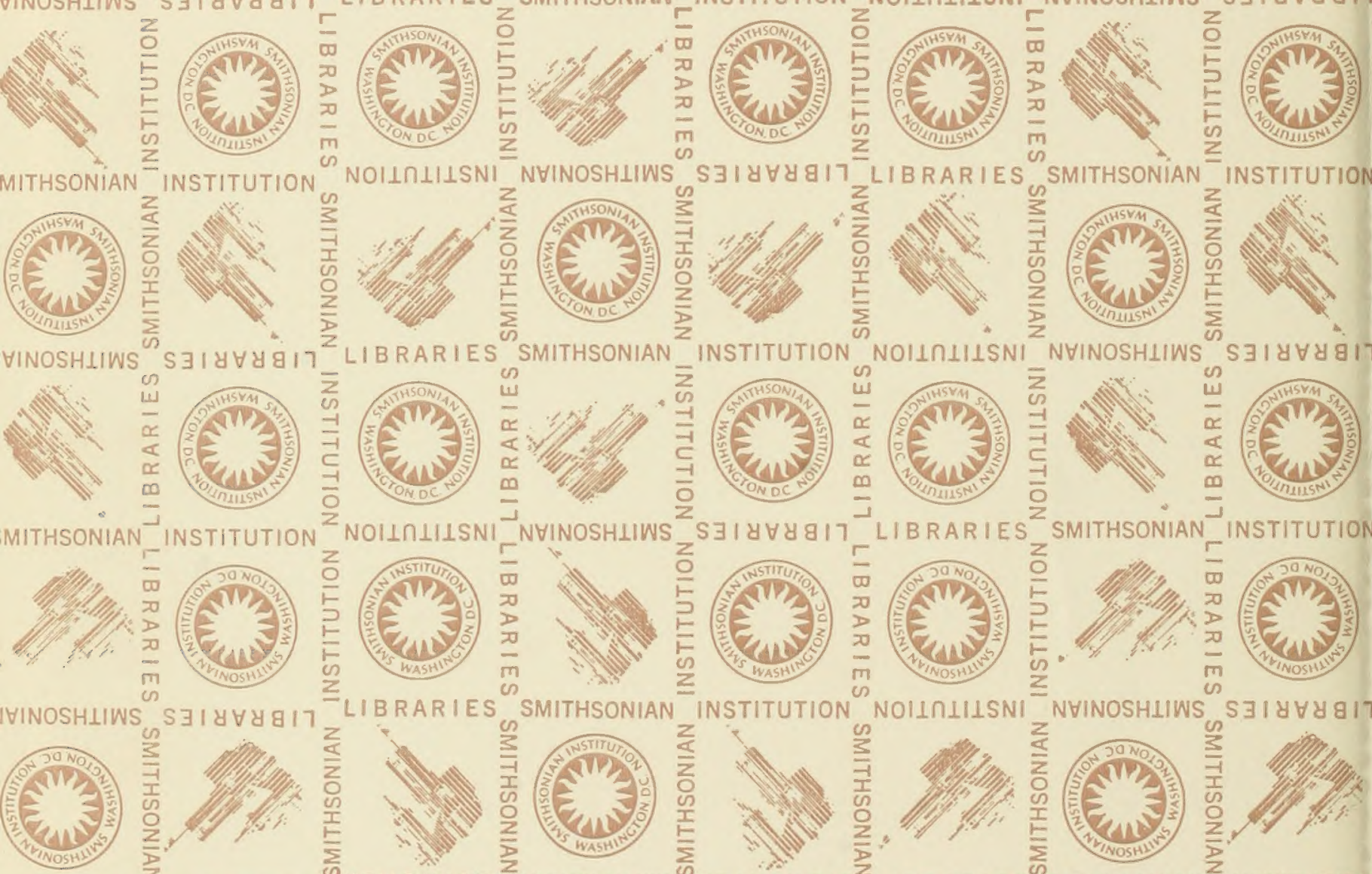

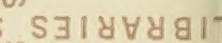

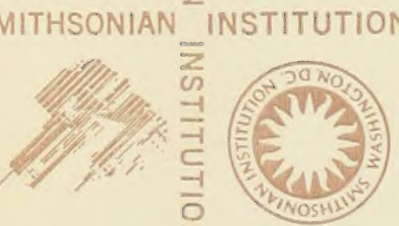

$$
\sum_{\text {"NOINLIISNI }}
$$
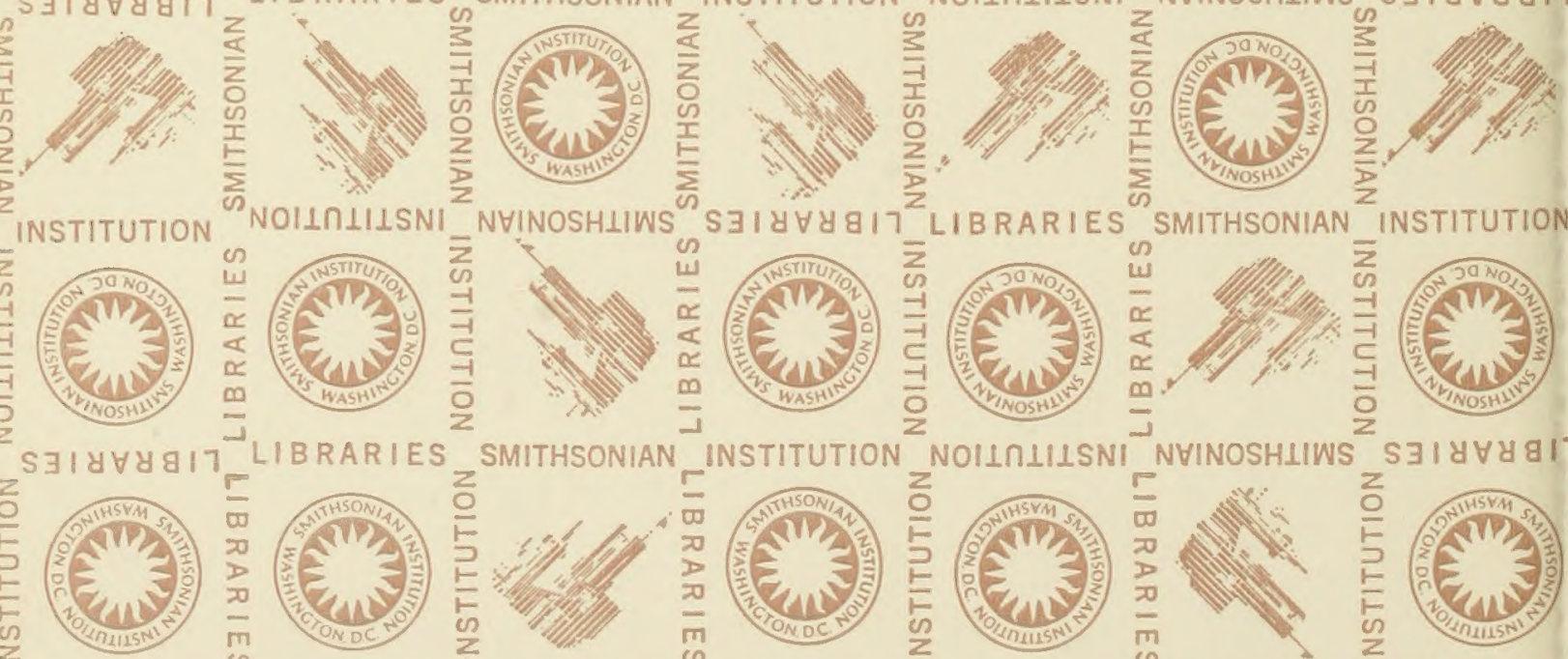

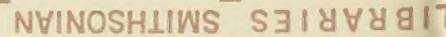

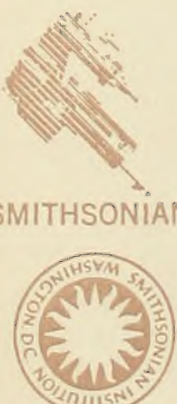

INSTITUTION
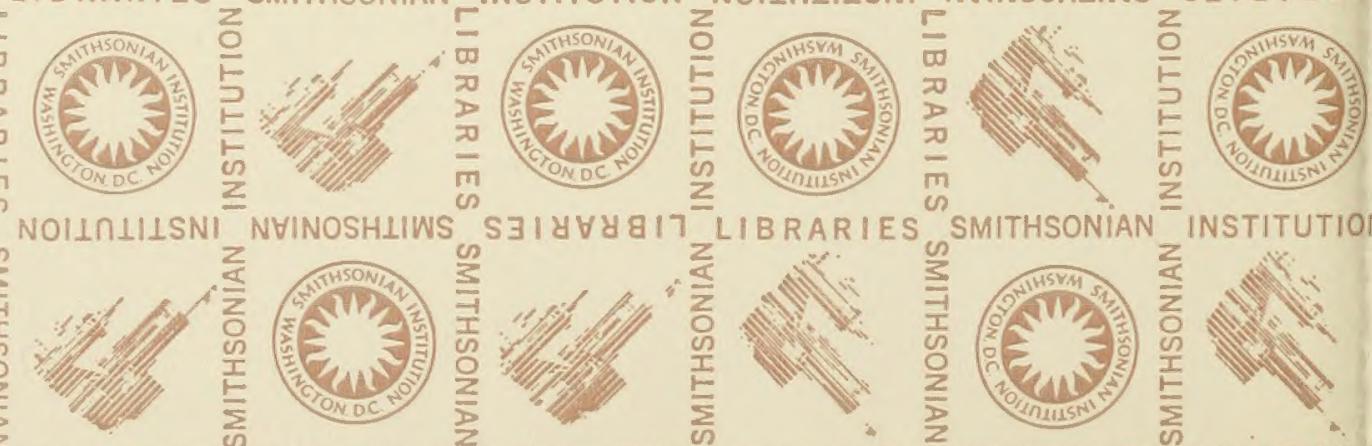

HINOSHLIWS ${ }^{\text {क }}$ SIIYHY $177^{z}$ LIBRARIES SMITHSONIAN

INSTITUTION NOILIIISNI
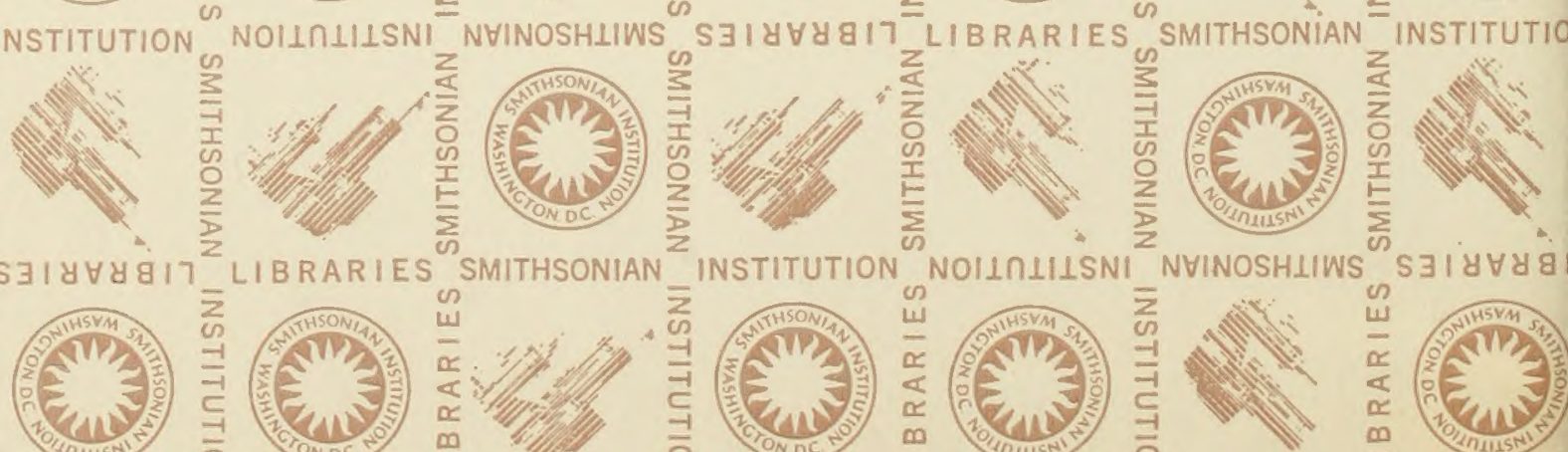

$\bar{z}$
n
$\overline{-1}$
$\bar{c}$
$\bar{n}$

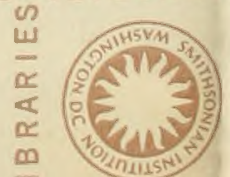


SMITHSONIAN INSTITUTION LIBRARIES 